Portland State University

PDXScholar

Summer 7-23-2018

\title{
Gendered Partner-Ideals, Relationship Satisfaction, and Intimate Partner Violence
}

Sylvia Marie Ferguson Kidder

Portland State University

Follow this and additional works at: https://pdxscholar.library.pdx.edu/open_access_etds

Part of the Domestic and Intimate Partner Violence Commons, and the Psychology Commons Let us know how access to this document benefits you.

\section{Recommended Citation}

Kidder, Sylvia Marie Ferguson, "Gendered Partner-Ideals, Relationship Satisfaction, and Intimate Partner Violence" (2018). Dissertations and Theses. Paper 4513.

https://doi.org/10.15760/etd.6397

This Dissertation is brought to you for free and open access. It has been accepted for inclusion in Dissertations and Theses by an authorized administrator of PDXScholar. Please contact us if we can make this document more accessible: pdxscholar@pdx.edu. 
Gendered Partner-Ideals, Relationship Satisfaction, and Intimate Partner Violence

by

Sylvia Marie Ferguson Kidder

A dissertation submitted in partial fulfillment of the requirements for the degree of

Doctor of Philosophy

in

Applied Psychology

\section{Dissertation Committee: \\ Eric Mankowski, Chair \\ Cynthia Mohr \\ Joel Steele \\ Maura Kelly}

Portland State University

2018 
(C) 2018 Sylvia Marie Ferguson Kidder 


\begin{abstract}
This dissertation identified and developed indicators of a new potential predictor of relationship satisfaction and intimate partner violence (IPV): discrepancies between the ideal and perceived gendered characteristics of romantic partners. Past research has overlooked the implicitly gendered nature of these "partner-ideals." Two pilot studies were conducted to develop measures of gendered partner-ideals and -perceptions based on existing measures of gender ideology.
\end{abstract}

The main study examined survey data collected online from adults $(n=643)$ living in the U.S. who were in a heterosexual romantic relationship for at least six months. Three main hypotheses were tested regarding the associations among gender ideology, gendered partner-ideals, gendered partner-perceptions, gendered partner-ideal discrepancies, relationship satisfaction, and experiences with IPV.

While confirmatory factor analyses supported the reliability of the new measures of masculine-ideals and -perceptions, results did not support the hypothesized factor structure of the feminine measures. Thus, only hypotheses utilizing women's data were tested. This measurement limitation resulted in an unanticipated focus on women's IPV perpetration for Hypothesis Two. However, these data are valuable in their uniqueness.

Hypothesis One was supported: women's masculinity ideology positively correlated with the corresponding masculine-ideal for each subscale of the respective measures as well as for the aggregate measures. Tests of Hypothesis Two showed that women's masculine-ideal discrepancies predicted their emotional abuse perpetration, but not their physical assault or injury perpetration, mediated by relationship satisfaction. 
However, this effect was small. Hypothesis Three had mixed support. Women's aggregate masculine-partner perceptions were positively associated with their reports of emotional abuse victimization. Additionally, women's reports of most types of IPV victimization positively correlated with perceptions of their male partners' conformity to the specific norms of negativity toward sexual minorities and restrictive emotionality.

Contributions of this dissertation and implications of the results are discussed. A major contribution is the creation of masculine-ideals and -perceptions measures that can be used in future research on relationships. Study results suggest that gendered partnerideals and -perceptions, beyond gender-ideology, have relevance to the functioning of romantic relationships - including both relationship satisfaction and some kinds of IPV. Future research should continue to investigate the validity of the created measures and explore the possibility of using discrepancies between gendered partner-ideals and perceptions to prevent and intervene in abusive romantic relationships. 


\section{Dedication}

For my beloved husband, Sam. 


\section{Acknowledgements}

This manuscript is a product of the effort, passion, and insight of many individuals. First, I would like to thank my committee for their expertise and support along the way: Dr. Cynthia Mohr, who introduced me to the study of dynamics within romantic relationships; Dr. Joel Steele, who challenged me to consider different perspectives on statistics; and Dr. Maura Kelly, who generously stepped in at the last moment to serve as my Graduate Office representative. While serving as my original Graduate Office representative, Dr. Emily Shafer reminded me to consider the broader context and implications of my research. Special thanks to my mentor and committee chair, Dr. Eric Mankowski, who spent countless hours discussing ideas and brainstorming solutions, polishing grant applications, and reviewing drafts. I take inspiration from his high ethical standards and commitment to socially-relevant research.

My graduate cohort and husband were instrumental in the early stages of this project. Ideas are relatively easy to come by, but these folks helped me figure out which ideas were worth pursuing. Once I had a firm grasp on my topic, my lab mates and other graduate students were great resources for shaping the measures I created and pointing me toward articles I never knew I should be reading. It would be impossible to name everyone who helped, but I am especially grateful to Jean McMahon, Kate Sackett, Jason Kyler-Yano, and Nicholas Smith. Even my cats, Merry and Pippin, contributed to the writing in their own way (i.e., probably a typo or two).

I thank my participants for the time and emotional energy they invested in completing my questionnaires. I was tickled to learn that some participants found it 
rewarding to reflect upon how their romantic relationships have grown and changed over time. I hope that others found it useful to consider the extent to which their romantic relationships are meeting their needs.

Lastly, I thank everyone who works (in research, practice, or simply in their own relationships) to promote healthy romantic relationships and reduce intimate partner violence. I took on this project because I believe it represents a small step toward a happier, safer world. 


\section{Table of Contents}

$\begin{array}{lc}\text { Abstract } & \text { i } \\ \text { Dedication } & \text { iii } \\ \text { Acknowledgements } & \text { iv } \\ \text { List of Tables } & \text { x } \\ \text { List of Figures } & \text { xi }\end{array}$

Chapter 1. Introduction 1

Manuscript Overview 1

Gender Role Ideology 4

Relationship between masculinity and femininity ideology 6

Domains of masculinity ideology $\quad 8$

Domains of femininity ideology $\quad 9$

Gender Role Conformity 11

Masculine role conformity 16

Feminine role conformity $\quad 21$

Gender and Romantic Relationships 26

Gender role conformity and relationships $\quad 27$

Partner-ideals and partner-ideal discrepancies $\quad 32$

Evolutionary explanations of partner-ideals $\quad 36$

Gender ideology and partner-ideals $\quad 39$

Gender ideology and relationship behaviors 45

Partner-ideal discrepancies and relationship satisfaction $\quad 50$

Intimate Partner Violence (IPV) 52

Theoretical perspectives of IPV $\quad 55$

Feminist perspective $\quad 55$

Psychological perspective $\quad 57$

IPV and gender ideology $\quad 61$

IPV, gender identity, and gender role conformity 67

$\begin{array}{ll}\text { IPV and relationship satisfaction } & 75\end{array}$

$\begin{array}{ll}\text { IPV and partner characteristics } & 78\end{array}$

Measurement of IPV $\quad 85$

Gaps in the Literature $\quad 89$

Chapter 2. Pilot Study to Develop Measures of Gendered Partner-ideals and 94

Partner-perceptions

Measure Development $\quad 94$

Hypotheses $\quad 96$

Hypothesis One $\quad 96$

Hypothesis Two $\quad 97$

Method

$\begin{array}{ll}\text { Participants } & 97\end{array}$

$\begin{array}{ll}\text { Measures } & 99\end{array}$ 
GENDER, RELATIONSHIP SATISFACTION, AND VIOLENCE

Feminine partner-ideals and partner-perceptions $\quad 99$

Masculine partner-ideals and partner-perceptions 99

Gendered partner-ideal discrepancies $\quad 99$

Relationship satisfaction 103

Demographics and relationship information 104

Data quality questions 104

$\begin{array}{ll}\text { Procedure } & 104\end{array}$

Results and Discussion $\quad 105$

$\begin{array}{ll}\text { Hypothesis One } & 105\end{array}$

$\begin{array}{ll}\text { Hypothesis Two } & 108\end{array}$

Chapter 3. Pilot Study to Examine Factor Structure of Measures of Gendered $\quad 110$

Partner-ideals and Partner-perceptions

Hypotheses 111

Hypothesis One $\quad 111$

Hypothesis Two 111

Hypothesis Three $\quad 112$

Method

112

$\begin{array}{ll}\text { Participants } & 112\end{array}$

$\begin{array}{lr}\text { Measures } & 116\end{array}$

Feminine partner-ideals and partner-perceptions $\quad 116$

Masculine partner-ideals and partner-perceptions $\quad 116$

Gendered partner-ideal discrepancies $\quad 117$

Relationship satisfaction $\quad 117$

Demographics and relationship information 119

Data quality questions $\quad 119$

$\begin{array}{lr}\text { Procedure } & 119\end{array}$

Data screening $\quad 120$

Results and Discussion $\quad 120$

$\begin{array}{lr}\text { Hypothesis One } & 120\end{array}$

Hypothesis Two 132

$\begin{array}{ll}\text { Hypothesis Three } & 136\end{array}$

Chapter 4. The Present Study: Associations between Gendered Partner-ideal 138

Discrepancies, Relationship Satisfaction, and IPV

$\begin{array}{ll}\text { Overview } & 138\end{array}$

$\begin{array}{ll}\text { Hypotheses } & 138\end{array}$

Hypothesis One 139

Hypothesis Two 139

$\begin{array}{ll}\text { Hypothesis Three } & 141\end{array}$

A Priori Power Analysis and Sample Size Justification $\quad 141$

Method

145

$\begin{array}{ll}\text { Participants } & 145\end{array}$

$\begin{array}{ll}\text { Measures } & 148\end{array}$ 
$\begin{array}{ll}\text { Partner-gender ideology } & 148\end{array}$

Femininity ideology 148

Masculinity ideology 149

Gendered partner-ideals, partner-perceptions, and partner-ideal 150 discrepancies

Feminine partner-ideals, partner-perceptions, and $\quad 150$

partner-ideal discrepancies

Masculine partner-ideals, partner-perceptions, and 151 partner-ideal discrepancies

Relationship satisfaction $\quad 153$

IPV 153

Physical violence $\quad 154$

Emotional abuse 155

Demographics and relationship/household characteristics 158

Data quality questions $\quad 158$

Procedure $\quad 158$

Data preparation and screening $\quad 159$

Dyad matching 159

Data screening $\quad 160$

Results

162

Preliminary analyses: Measures of feminine and masculine partner- 163 ideals

CFA of feminine-ideals and-perceptions measures $\quad 163$

CFA of masculine-ideals and-perceptions measures 168

Descriptive and comparative data 173

Checking statistical assumptions for hypothesis tests $\quad 174$

Hypothesis One 178

Hypothesis Two 180

Hypothesis Three 184

Post hoc analyses 186

Masculine-perceptions and relationship satisfaction $\quad 186$

$\begin{array}{ll}I P V \text { and relationship satisfaction } & 187\end{array}$

Chapter 5. Discussion, Implications, and Limitations 189

Implications for Theory and Research 189

Measure development 190

Partner-ideals 192

Masculine partner-perceptions 195

Partner-ideal discrepancies $\quad 197$

IPV 200

$\begin{array}{ll}\text { Limitations } & 209\end{array}$

Study design 209

Measures 211

Sample and data collection 213 
Analysis 214

Conclusions

$\begin{array}{ll}\text { References } & 252\end{array}$

Appendices 268

Appendix A: Accessing the Partner-ideals and Partner-Perceptions $\quad 268$ Measures

Appendix B: Pilot Study Demographic and Survey Quality Questions 269

Appendix C: First Pilot Study Consent Form 273

Appendix D: Pilot Study Eligibility Screening Questions 274

Appendix E: Second Pilot Study Eligibility Screening Questions 275

Appendix F: Second Pilot Study Consent Form 276

Appendix G: Demographic Information 277

Appendix H: Relationship Characteristics 279

Appendix I: Data Quality Questions, Invitation for Partner Survey, and 280

Compensation Directions

Appendix J: Eligibility Screening Pre-Questionnaire 282

Appendix K: MTurk Participant Consent Form 283

Appendix L: Partner Survey Consent Form 285

Appendix M: Partner Survey Data Quality Questions and Compensation 287

Directions 


\section{List of Tables}

Table 1. First pilot study feminine partner-ideals and-perceptions descriptive statistics and subscale intercorrelations

Table 2. First pilot study masculine partner-ideals and-perceptions descriptive 218 statistics and subscale intercorrelations

Table 3. First pilot study Hypothesis 2: Correlations between partner-ideal discrepancies and relationship satisfaction

Table 4. Second pilot study feminine partner-ideals and -perceptions subscale descriptive statistics and correlations

Table 5. Second pilot study feminine partner-ideals and -perceptions factor loadings

Table 6. Second pilot study feminine partner-ideals and -perceptions latent factor correlations

Table 7. Second pilot study masculine partner-ideals and-perceptions subscale descriptive statistics and correlations

Table 8. Second pilot study masculine partner-ideals and-perceptions factor loadings

Table 9. Second pilot study masculine partner-ideals and-perceptions latent factor correlations

Table 10. FIS descriptive statistics and subscale intercorrelations

Table 11. MRNI-SF descriptive statistics and subscale intercorrelations

Table 12. Feminine partner-ideals and-perceptions descriptive statistics and subscale intercorrelations

Table 13. Masculine partner-ideals and-perceptions descriptive statistics and subscale intercorrelations

Table 14. PRQC descriptive statistics and subscale intercorrelations by gender

Table 15. CTS2 descriptive statistics and subscale intercorrelations for female participants

Table 16. CTS2 descriptive statistics and subscale intercorrelations for male participants

Table 17. MMEA descriptive statistics and subscale intercorrelations for female participants

Table 18. MMEA descriptive statistics and subscale intercorrelations for male participants

Table 19. CFA: Feminine partner-ideals and -perceptions factor loadings

Table 20. CFA: Feminine partner-ideals and-perceptions latent factor correlations

Table 21. Comparison of alternative feminine partner-ideals and-perceptions models

Table 22. CFA: Masculine partner-ideals and - perceptions factor loadings

Table 23. CFA: Masculine partner-ideals and-perceptions latent factor correlations

Table 24. Comparison of alternative masculine partner-ideals and -perceptions 
models

Table 25. Hypotheses 1c and 1d: Correlations between masculinity ideology and masculine partner-ideals among female participants

Table 26. Hypothesis 2a: Mediation models testing effect of women's masculine partner-ideal discrepancies on physical intimate partner violence perpetration via relationship satisfaction

Table 27. Hypothesis 2b: Mediation models testing the effect of women's masculine partner-ideal discrepancies on emotional abuse perpetration via relationship satisfaction

Table 28. Hypotheses 3a and 3b: Correlations between men's gender role conformity and IPV perpetration

Table 29. Post hoc analyses on IPV: Bivariate correlations between PRQC mean scores, masculine partner-ideal discrepancies, and IPV perpetration/victimization

Table 30. Summary of results of hypothesis tests 


\section{List of Figures}

Figure 1. The proposed moderated mediation model for Hypothesis 2a

Figure 2. The proposed moderated mediation model for Hypothesis $2 b$

Figure 3. Hypothesis 2a: Results of the mediation model for women's physical assault perpetration

Figure 4. Hypothesis 2a: Results of the mediation model for women's injury perpetration

Figure 5. Hypothesis 2b: Results of the mediation model for women's emotional 251 abuse perpetration 


\section{Chapter 1. Introduction}

\section{Manuscript Overview}

The purpose of this project was to identify and develop indicators of a new potential predictor of relationship satisfaction and intimate partner violence (IPV): discrepancies between the ideal and perceived gendered characteristics of romantic partners. Individuals in romantic relationships were recruited to complete an online survey containing measures of gender ideology, gendered partner-ideals, gendered partner-perceptions, relationship satisfaction, and experiences with IPV (including both perpetration and victimization). Gender ideology was explored as a predictor of gendered partner-ideals, and the association between men's perceived conformity to gender role norms and IPV perpetration was also examined. Additionally, I modeled the association between women's masculine partner-ideal discrepancies and IPV, mediated by the association between masculine-ideal discrepancies and relationship satisfaction.

While IPV has long been studied within the context of gender role norms, the causal relationship between these norms and IPV is still unclear. Gendered partner-ideals represent a potential mechanism by which gender ideology could impact relationship satisfaction and, more distally, IPV. To lay the groundwork for the examination of gendered partner-ideals, this dissertation manuscript begins with a review of the history of gender role ideology. In this section, the components of male and female gender roles, respectively, are described. Having introduced the concept of traditional gender ideology, the document next describes gender role conformity, or adherence to traditional gender role norms. Gender role conformity is differentiated from gender ideology. Advances in 
the conceptualization and measurement of gender role conformity are described. After considering different motivations to conform, I describe the outcomes of fulfilling —or failing to fulfill — gender roles. Readers will note that conformity to the traditional male role is linked to many negative outcomes. There is relatively little research, however, on the outcomes of conformity to the female role.

Next, gender ideology and gender role conformity are considered in the context of romantic relationships. Research on gender role conformity in relationships suggests that the impact of conformity to particular norms on relationship outcomes is mediated by their association with positive or negative relationship behaviors. Norms associated with negative relationship behaviors (e.g., conflict) predict poor relational outcomes, whereas those associated with positive relationship behaviors (e.g., affection) predict good relational outcomes. The literature also demonstrates that relationship outcomes are predicted by partner perceptions of gender role conformity, not just self-reported conformity. Here I introduce the concept of partner-ideals in romantic relationships. The structure and function of these ideals are briefly summarized. I explain how past research on partner-ideals has largely taken an evolutionary perspective. Then, I present the evidence that gender differences in partner-ideals are largely influenced by socialization. Specifically, research consistently supports a positive relationship between gender ideology and stereotypically sex-typed partner-ideals. Then, I describe how gender ideology influences relationship satisfaction and relational outcomes; people are more satisfied to the extent that their actual roles (e.g., breadwinning, engagement in domestic labor) match their gender ideology. I explain how partner-ideal discrepancies negatively 
predict relationship satisfaction and also motivate various behaviors to attempt to decrease those discrepancies.

In the last major section of the literature review, I introduce IPV as a relationship behavior that represents a potential strategy to reduce gendered partner idealdiscrepancies. IPV is defined, and various theoretical frameworks are summarized. Most past research has found that holding a traditional gender ideology increases the likelihood of perpetrating IPV. However, more recent studies have found that the gender ideologies of husbands and wives interact to predict IPV. I offer the interpretation that IPV in such situations could be, in fact, a reaction to a mismatch between gender role expectations and reality. Gender role conformity, apart from role expectations, is then explored as a predictor of IPV. The bulk of the research finds that adherence to certain male role norms predicts increased aggression in both men and women, while there are some studies suggesting that women's feminine role conformity is protective against victimization. I also describe the limited research on partner characteristics and IPV to underscore the need for more research on gender role conformity, partner-ideals, and IPV. Relationship satisfaction is also considered as a potential contributor to and outcome of IPV; I note that there is uncertainty regarding the direction of causality.

After reviewing the relevant literature, I point out weaknesses with and gaps in previous research and describe how they are addressed by the present study. One important weakness involves the inadequate measurement of gendered partner-ideals. This discussion leads to Chapter 2, in which I describe a small pilot study I conducted on new measures of feminine and masculine partner-ideals that were developed using 
existing measures of gender ideology. Complementary measures of perceived partner gender role conformity were also created to allow for the calculation of gendered partnerideal discrepancies.

Chapter 3 details a second, larger pilot study on the new measures of feminine and masculine partner-ideals and-perceptions. The factor structure of each measure is analyzed. Additionally, the negative correlation between partner-ideal discrepancies and relationship satisfaction is confirmed.

In Chapter 4, I give a brief overview of the main study and outline the specific hypotheses tested. The methods section describes the study sample, the measures that were administered (including the process for creating a measure of gendered partnerideals and ethical considerations for the instrument assessing IPV), and the process for collecting data online. I specify the analyses that were used to the hypotheses and provide the results obtained. The conclusions and implications of the results are described in Chapter 5. The document ends with a discussion of the study's limitations.

\section{Gender Role Ideology}

Whereas "sex" refers to an individual's biological status as male, female, or intersex, "gender" describes the attitudes, feelings, and behaviors usually associated with each sex. Because ideas of what is natural and acceptable for each sex varies across time and place, gender is socially determined. Gender roles are comprised of the social norms, or culturally acceptable and desirable attributes, for each gender. Social norms range from cognitive (e.g., highly valuing relationships) to behavioral tendencies (e.g., taking risks). 
While the term "gender roles" is often used in a way that makes these roles seem both static and universally accepted, at least within a given culture, neither is necessarily true. Not only can there be disagreement about the appropriate content of gender roles, but there is also substantial variation in the degree to which individuals personally value these roles. "Gender ideology" represents beliefs about the importance of adhering to those gender roles (Pleck, Sonenstein, \& Ku, 1998).

Gender ideology is also commonly called "attitudes toward male/female gender roles" or "attitudes toward masculinity/femininity." Pleck and colleagues (1998) have explained that it is preferable to frame this concept as an ideology rather than an attitude for at least two reasons. First, gender roles are different than most attitude objects; while people may feel favorably or unfavorably about the masculine or feminine role, they do not enact behaviors toward these roles in the traditional sense of an attitude object. Second, the term "ideology" better conveys the endorsement and internalization of cultural belief systems about gender. In other words, it draws attention to the larger social context of these beliefs.

I would add that using the term "ideology" also differentiates this concept from descriptive beliefs about men and women that are conceptually related to, yet distinct from, gender ideology. For example, sexism describes prejudice, stereotyping, or discrimination on the basis of gender. Sexist stereotypes, such as that women are overly emotional or possess innate moral superiority to men, are different from the ideological beliefs that women should be, for example, emotionally available and nurturing. While highly compatible, these beliefs are not mutually inclusive. One may think that women 
as a group are overly emotional—a sexist stereotype—and simultaneously believe (or not) that women should be calm and stoic - representing a nontraditional gender ideology. In other words, gender ideology represents injunctive norms rather than descriptive norms (Cialdini \& Trost, 1998).

Relationship between masculinity and femininity ideology. "Gender ideology" refers to global concepts of gender. Practically, these concepts are usually limited to social norms for women and men. "Femininity ideology" is specific to female roles and "masculinity ideology" to male roles. However, as previously noted, biological sex is not binary. Accordingly, individuals' gender identity, or personal experience of their own gender, is not restricted to "woman" or "man." Identities such as agender (having no gender), androgyne (having both masculine and feminine aspects), and genderqueer (having a gender identity outside of the male/female binary) are becoming increasingly visible, as is reflected in the recent research interest in determining how best to measure non-binary gender identities (Richards et al., 2015). Nevertheless, mainstream American culture continues to recognize only masculine and feminine norms. Because there are no widely-accepted sets of norms for non-binary genders, they do not fall under the larger umbrella of gender ideology. For example, there is no meaningful "androgyny ideology" within gender ideology.

Just as American society is slowly, and only recently, beginning to accept nonbinary genders as legitimate, so psychological theory and measurement for non-binary genders has lagged behind. By necessity, the scope of the present research is restricted to masculinity and femininity ideology. This decision is purely practical and should not be 
interpreted as an endorsement of a binary gender system. There are no measures of nonbinary gender ideologies, and I lack the resources to properly develop new measures for this study. Recruitment of individuals with non-binary gender identities would also cause analytical difficulties, with those analyses that would be theoretically possible most likely lacking the statistical power to enable meaningful conclusions. Similarly, this study's focus on dyadic romantic relationships is one of practicality and not meant to delegitimize other kinds of relationships such as polyamory.

Gender has long been conceptualized as binary (i.e., consisting of two separate entities) or even bipolar (i.e., comprising opposite ends of the same spectrum). However, ideas concerning the relationship of masculinity and femininity — and, by extension, masculinity and femininity ideologies - to each other are gradually evolving. One of the major stepping-stones in measuring gender ideology was Spence and Helmreich's stillpopular Attitudes Toward Women Scale (AWS; 1972). This instrument measures attitudes toward women's roles in society, with the majority of items focusing on appropriate career, domestic, and marital roles. Importantly, this scale includes explicit comparisons to men's roles. Some questions are double-barreled, asking about women's and men's roles simultaneously, and others focus solely on men (e.g., "The intellectual leadership of a community should be largely in the hands of men"). Thus, this scale reflects an assumption of the complementarity of women's and men's roles: women's place is in the domestic sphere, while men belong in the workplace. Disagreement with the appropriateness of this division is compatible with a progressive gender ideology (i.e., the belief that it is unimportant to conform to gender role norms). However, it is more 
accurately described as egalitarianism, or the belief that men and women should have equal rights and treatment in all areas of activity, than progressive gender ideology.

Despite the distinction between progressive gender ideology and egalitarianism, traditional gender ideology is still commonly operationalized as an acceptance of the complementary gendered division of labor. For example, a relatively recent review of work on gender ideology defined it as "individuals' levels of support for a division of paid work and family responsibilities that is based on [the] notion of separate spheres" (Davis \& Greenstein, 2009, p. 88). However, gender ideology expands far beyond the division of labor; traditional roles for each gender are comprised of unique constellations of attributes. The individual domains of masculinity and femininity ideology, respectively, are described in the following two subsections. First, though, it is important to point out that not all gender role norms are complementary. For example, while men are traditionally expected to display subtle or even overt homophobia, women's presentation of sexual orientation is less strictly regulated (e.g., Herek, 2000). Thus, when measuring gender ideology, it is critical to examine endorsement of masculine and feminine role norms separately.

Domains of masculinity ideology. Conceptualizations of the structure of male role norms — and thus the content of masculinity ideology—have changed and expanded over time. Some of the earliest theories simply stated that men are expected to be masculine and to avoid being feminine (Thompson \& Pleck, 1986). Subsequent models retained avoidance of femininity as part of masculinity but identified the additional 
domains of achievement and status, independence and self-confidence, restrictive emotionality, and aggressiveness (Levant et al., 1992; Thompson \& Pleck, 1986).

The most reliable and valid scale of masculinity ideology currently available is the revised Male Role Norms Inventory (MRNI-R; see the Measures section for more details; Levant \& Richmond, 2007; Levant et al., 2007b), which identifies seven male role norms: avoidance of femininity, fear and hatred of homosexuals, extreme selfreliance, aggression, dominance, non-relational sexuality, and restrictive emotionality. Avoidance of femininity involves eschewing behaviors and signifiers stereotypically associated with women, such as watching soap operas or wearing makeup. Fear and hatred of homosexuals includes both prejudicial attitudes toward homosexuals (specifically men) and fear of appearing gay to others. Extreme self-reliance involves general self-sufficiency as well as mechanical and home repair skills. Aggression includes elements of risk-taking, physical prowess, and protecting others from harm. Dominance, which evolved from an earlier concept called "achievement and status," primarily focuses on leadership and decision-making. Non-relational sexuality represents preoccupation with and a sense of entitlement to sex, which traditionally centers on the male orgasm to the exclusion of intimacy and the pleasure of one's partner. Restrictive emotionality focuses on maintaining a stoic façade regardless of one's true feelings.

Domains of femininity ideology. As previously mentioned, femininity ideology is frequently conflated with attitudes towards women's roles in comparison to men's. Only recently have researchers begun to identify the individual domains of femininity ideology. In their validation of the Femininity Ideology Scale (FIS), Levant, Richmond, 
Cook, House, and Aupont (2007) confirmed the presence of five factors: stereotypic image and activities, dependence/deference, purity, caretaking, and emotionality. The first factor focuses on traditional markers of attractiveness such as being petite and wearing attractive clothing. Dependency/deference concerns women's deference to her romantic partner and the deprioritization of her career. Purity involves both chastity and traditional morality. Caretaking is equivalent to nurturance. Emotionality primarily focuses on stereotypic over-emotionality (e.g., inability to handle stress, irrationality). This last factor is unusual among gender role norms because over-emotionality is generally considered an undesirable attribute.

Because the FIS is relatively new, there is little descriptive data available. However, it is known that scores on the FIS are positively and moderately correlated with those on the MRNI (Levant et al., 2007a). This means that to the extent an individual endorses traditional femininity ideology, they also tend to endorse traditional masculinity ideology and vice versa. However, there are main effects of gender and age on the endorsement of traditional gender ideology. Men tend to endorse both masculinity and femininity ideology more strongly than do women (Levant et al., 2007a). Some have suggested that this endorsement is motivated by traditional femininity ideology's contribution to women's systemic subordination (e.g., Levant et al., 2007a). Young adults, compared to people aged 55 or older, also endorse both traditional masculinity and femininity ideology more strongly (Gronemeyer, 1998). Despite these group differences, femininity ideology is generally weakly endorsed, with mean scores trending slightly toward disagreement (Levant et al., 2007a; Richmond et al., 2015). 


\section{Gender Role Conformity}

If gender ideology involves endorsement of cultural standards for the behavior of men and women, then "gender role conformity" represents behavioral adherence to those standards. Gender role conformity is also frequently referred to as "gender conformity" (e.g., Good \& Sanchez, 2010; Sears, 2011), “gender (stereo)typicality” (e.g., Jewell \& Brown, 2014; Smith \& Leaper, 2005; Young \& Sweeting, 2004), and occasionally just “masculinity/femininity" (although research using this last term tends to use a simplified schematic of gender roles; e.g., Aube \& Koestner, 1995; Frazier \& Esterly, 1990; Lamke, Sollie, Durbin, \& Fitzpatrick, 1994; Siavelis \& Lamke, 1992). Mahalik and colleagues (2003) defined gender role conformity as "meeting societal expectations for what constitutes masculinity [or femininity] in one's public or private life" (p. 3). Thus, gender role conformity is not restricted to public or even outwardly detectable behavior. Rather, it can also include adherence to gender role norms in the areas of attitudes, beliefs, personality, emotional responses, and physical attributes.

Despite the fact that psychology typically uses "conformity" to imply that adherence to social norms is motivated by real or perceived social pressure (Cialdini \& Trost, 1998), the motivations behind gender role conformity vary. Deaux and Major's (1987) seminal model of gender-related behavior captures the variety of potential influences on gender role conformity. This model states that an individual's level of gender role conformity in a given situation is determined by a complex interaction between the expectations of perceivers, the self-systems of the individual, and situational cues that vary in the extent to which they make gender salient. In other words, gender 
role conformity is not simply a characteristic possessed by actors but rather is produced through social interactions. Thus, an individual's degree of gender role conformity is flexible and may vary across contexts.

In this model, one individual (arbitrarily distinguished as the "perceiver") holds a particular gender ideology and descriptive beliefs about gender. Depending on the chronic accessibility of those beliefs, elements of the situation, and the information the perceiver has about the "target" (the interactional partner), the perceiver will hold expectations for the target. For example, a heterosexual man with traditional views about gender may encounter a stereotypically feminine-appearing woman at a singles mixer. With the combination of his internal beliefs, the target's apparent attributes, and the clear behavioral scripts relevant to this particular situation, gender is likely to be highly salient to him. Thus, he will probably hold strong expectations that the woman will behave in a stereotypically feminine way. At the same time, the target possesses self-schemas, which likely include gender identity (i.e., the sense of oneself as a man or woman) as a key organizing dimension. Even if the woman in this example does not hold gender as a particularly important part of her identity, the situation is likely to make it salient. This would be especially true if the perceiver acts in such a way that makes it salient, such as by complimenting some feminine attribute.

Informed by their belief systems, situational cues, and specific interactional goals, the perceiver and target each act. According to the model, a target will be especially motivated to confirm the perceiver's expectancies to the extent that they represent socially desirable behaviors, are held with high certainty by either interactional partner, 
and align with strong situational cues. Throughout the interaction, the target and perceiver interpret each others' actions. These interpretations may, in turn, feed back into beliefs regarding gender and views of the self.

Deaux and Major's (1987) model of gender-related behavior refutes the idea that gender role conformity derives primarily from an individual's acceptance of the role norms for his or her gender. If gender role conformity were motivated only by internalized social standards, then the association between gender ideology and gender role conformity should be nearly perfect. In fact, there are only moderate correlations between the various domains of gender ideology and gender role conformity (Sears, 2011). This finding is congruent with the idea that the social context—including situational cues and the expectations of others-is a major source of influence on gender role conforming behaviors.

As with other social norms, the social context frequently offers rewards for gender role conformity as well as punishment for nonconformity. The negative social and economic consequences of engaging in counterstereotypic behavior-including gender role nonconformity—are called "backlash" (Rudman, 1998). Social consequences could vary widely depending on the actor and the relational context, ranging from mild (e.g., decreased warmth and liking) to severe (e.g., violence). For example, snubbing would probably be a relatively common form of backlash in the workplace, whereas physical violence is more likely to occur in romantic relationships.

Backlash contributes to the maintenance of stereotypic behavior through its effects on both target and perpetrator. For the target, the threat of backlash can motivate 
subsequent gender role conformity as well as attempts to hide or inaccurately report one's own nonconformity (Rudman \& Fairchild, 2004). While perpetrators of backlash frequently experience a boost in self-esteem, nonconformity can result in decreased selfesteem through the fear of backlash (Rudman \& Fairchild, 2004).

However, the effect of gender conformity or nonconformity on self-esteem appears to depend on both individual factors and perceptions of the social context. Wood et al. (1997) found that people who valued fulfilling gender role norms or who believed that gender role norms were highly valued in society experienced positive affect and a more positive self-concept after recalling an interaction in which they had behaved in a gender-congruent manner (i.e., communally for women and agentically for men). Thus, gender conformity seems to offer benefits when it is an authentic expression of the self or when it is believed to be valued by others.

Good and Sanchez (2010) further elucidated the role of motivation in the impact of gender conformity. They determined that investment in gender ideals (i.e., the extent to which individuals found it important to be similar to the ideal member of their gender) predicted external motivation to conform to gender role norms, or feeling pressured to engage in gender normative behavior because of others' expectations. External motivation in turn negatively predicted self-esteem. In contrast, positively valuing one's gender and gender identity resulted in internal motivation for gender conformity and subsequent increases in self-esteem. The two sources of motivation were moderately correlated, showing that there can be multiple simultaneous motivations for gender role conformity. 
The results of Wood et al. (1997) and Good and Sanchez (2010) make it clear that the actual and anticipated reactions of other people influence gender role conformity. Most of the research on reactions to gender role conformity and nonconformity, however, has been limited to hypothetical, casual, or work-based relationships. Romantic relationships are notably absent. Some research has been conducted on the association of stereotypically masculine and feminine traits (i.e., agency and communion, respectively) with romantic relationship outcomes, but there is dearth of information on other domains of gender role conformity. It is unclear what pressures to conform and what consequences of gender role nonconformity exist within romantic relationships.

The present research begins to address this gap in these topics by examining the potential influence of romantic partners' perceived gender role conformity on intimate partner violence (IPV). As individuals vary in their endorsement of traditional gender ideology, it seems reasonable to expect corresponding variation in the gendered expectations individuals hold for their romantic partners. There may be relationshipspecific forms of backlash for gender role nonconformity, such as verbal conflict or violence. The severity of relationship-specific backlash could also depend on the personal importance of conformity to specific gender role norms. To illustrate, a woman may engage in backlash behaviors toward her male partner if she perceives him as failing to be high status, but only if she values that specific characteristic in a romantic partner. Before identifying outcomes of gender nonconformity, though, we must understand the different domains of gender conformity. 
In previous sections, I have noted that there are different sets of expectations for men and women. Thus, there are a variety of domains in which one can conform or violate the norms of one's gender. The subsections below explore research on conformity to masculine and feminine roles, respectively. As the consequences of gender role nonconformity vary by gender, gender-specific backlash effects are also discussed.

Masculine role conformity. Researchers interested in the assessment of masculine role conformity started with previous models of traditional masculinity ideology including Levant et al.'s (1992) Male Role Norms Inventory and used focus groups to expand upon and refine the domains therein. The Conformity to Masculine Norms Inventory (CMNI), which identifies eleven areas of role conformity, was the first such instrument developed (Mahalik et al., 2003). Subsequent work on the factor structure resulted in the nine-factor model of the CMNI-46 (Parent \& Moradi, 2009). Within masculine role conformity are the distinct factors of emotional control, selfreliance, heterosexual self-presentation, playboy (having frequent casual sexual relationships), winning (competition), violence, primacy of work, risk-taking, and power over women. While there is substantial overlap between the domains of Levant, Hall, and Rankin's (2013) MRNI-SF and the CMNI-46, avoidance of femininity is unique to the MRNI-SF and the CMNI-46 adds the dimensions of primacy of work, risk-taking, and power over women. More research is needed to reconcile these differences.

It is important to point out that masculine role conformity as assessed with the CMNI-46 is not equivalent to high levels of trait masculinity. Recall that trait masculinity focuses only on agency, likely reflecting the fact that agency is implicit in 
several masculine role norms. It seems that as research moved further away from a bipolar perspective of gender, theorists were freed to identify role domains that lacked corollaries in the other gender. In fact, only the subscales of risk taking and winning predict men's stereotypically masculine personality traits (Smiler, 2006). In this subsection and the next, research operationalizing gender role conformity as trait masculinity/femininity is described as measuring conformity to "stereotypical traits/characteristics" to differentiate it from research using measures of conformity to distinct gender role norms (i.e., the CMNI and its female equivalent).

Research using the CMNI and CMNI-46 has shown that masculine role conformity is linked to unhealthy and problematic outcomes including depression (Rice, Fallon, \& Bambling, 2011; risky health behaviors (Mahalik, Burns, \& Syzdek, 2007), sexist attitudes (Smiler, 2006; Smiler \& Gelman, 2008), alcohol abuse (Khairallah, Treloar, \& McCarthy, 2010), and decreased life satisfaction (Rochlen, McKelley, Suizzo, \& Scaringi, 2008). Masculine role conformity also predicts relationship dissatisfaction and a propensity for violence, issues that are discussed in greater depth in later sections of this document. Many of these negative outcomes seem like natural extensions of specific role norms. For example, risk-taking should lead to riskier health behaviors, and aggressiveness should lead to violence. Others, however, likely have a more complex etiology. Decreased life satisfaction could be partially a result of difficulty forming strong social connections due to restrictive emotionality, for example.

However, there is also some evidence of positive correlates of masculine role conformity. Men's CMNI scores positively predict both preferences for and self-efficacy 
in stereotypically masculine occupations (Bogaert, Fawcett, \& Jamieson, 2009; Tokar, Thompson, Plaufcan, \& Williams, 2007). Scores also predict both self- and other-rated physical attractiveness (Bogaert et al., 2009), although these results could have been contaminated by interaction between the subject and rater. Many of these positive outcomes seem related to the agency underlying several masculine role norms.

Despite the numerous negative outcomes associated with masculine role conformity, men also experience backlash for failing to conform to masculine role norms. The nature of the backlash depends on the norm being violated. Most of the research on backlash against men's gender nonconformity has focused on the context of the workplace. One study found that men who violate norms of dominance by self-effacing are viewed as less competent and hirable, although they are also seen as more likeable than self-promoting men (Rudman, 1998). Even fulfilling a positively valued feminine norm, such as caretaking, can be harmful; men who request family leave from work are seen as not only weaker and more feminine but also as poorer workers than men who do not (Rudman \& Mescher, 2013). To the extent that they perceived this request as representing weakness, participants were likely to advocate penalization (e.g., demotions) and a lack of career rewards (e.g., recommendations for a leadership training program). Additionally, despite the male role norm of achievement, success in a traditionally feminine job leads to negative perceptions and decreased respect (Heilman \& Wallen, 2010).

Other research has examined how gender moderates the outcomes of certain characteristics, depending on whether the characteristic is normative for men or women. 
One study found that modest men (who violate dominance norms) are perceived as less likeable, though not less competent or hirable, than modest women (Moss-Racusin, Phelan, \& Rudman, 2010). Men who violate the norm of restrictive emotionality by crying in the workplace are seen as more emotional as well as less competent than women engaging in the same behavior (Fischer, Eagly, \& Oosterwijk, 2013). Thus, both men's careers and reputations could suffer as a result of masculine role nonconformity.

Several studies have suggested that men face harsher consequences than do women for failing to conform to the norms of their gender (cf. Rudman \& Fairchild, 2004; Rudman \& Glick, 2001). In their examination of both trait-based and behavioral violations of gendered norms, Sirin, McCreary, and Mahalik (2004) found that, compared to women described as having stereotypically masculine traits, men with feminine traits are perceived as lower in social status and as more likely to be homosexual. Men who were said to behave in gender nonconforming ways (e.g., avoiding behaviors that might anger another person, failing to defend oneself) were also perceived as lower-status than women who behaved in nonconforming ways. Men seem to be aware that they are likely to be judged harshly for nonconformity; under some circumstances, men are more afraid of potential backlash for gender nonconformity than are women (Rudman \& Fairchild, 2004). These fears could be partially based in the fact that compared to women, men are especially critical of nonconforming individuals (Sirin et al., 2004); perhaps they are expecting the same harsh critcism from others.

Backlash against masculine role nonconformity starts early. Starting in childhood, it is perceived particularly negatively and punished more severely than is feminine role 
nonconformity. In middle school, boys' masculinity gender typicality (i.e., having stereotypically masculine traits) is positively related to popularity and having more male friends, while it is negatively related to loneliness and psychological distress (Jewell \& Brown, 2014; Young \& Sweeting, 2004). Evidence suggests that the association between boys' gender atypicality and poorer mental health outcomes can be partially explained by vulnerability to teasing or bullying about these atypical traits. Adolescents' gender typicality positively predicts self-worth to the extent that they feel external pressure to conform to the norms of their gender (Smith \& Leaper, 2005), demonstrating again that external motivation (and not just gender ideology) exerts powerful influence on gender role conformity.

Many parents seem cognizant of the heightened pressures boys face to conform to masculine role norms. Not only do parents have more negative attitudes toward boys' than toward girls' gender nonconformity, but they also anticipate gender nonconforming children — especially boys — to have more psychological adjustment problems in adulthood (Martin, 1990; Sandnabba \& Ahlberg, 1999). These attitudes are expressed in parents' reactions to their own children's gendered behavior. One qualitative study of parents found that while many parents encouraged gender nonconformity in girls, attitudes toward boys' nonconformity were much more mixed (Kane, 2006). Most parents of boys responded negatively to at least a few "items, activities, or attributes that could be considered icons of femininity," such as feminine dress, Barbie dolls, and an interest in dance (Kane, 2006, p. 159). Several parents theorized that such feminine markers were indicators of - or could even somehow contribute to-a homosexual 
orientation, a prospect that fathers found particularly troublesome. Heterosexual fathers commonly felt personally responsible or even morally obligated to ensure that their sons fulfilled hegemonic ideals. They saw sons' gender nonconformity as reflecting poorly on themselves. In contrast, heterosexual mothers and homosexual parents of both sexes commonly expressed that their reactions to gender nonconformity were not necessarily an expression of their own gender ideologies. Rather, these parents were concerned with the social evaluations of peers and society at large. Thus, many parents, especially those of boys, may be paradoxically reinforcing pressures for gender conformity through their well-intentioned efforts to protect their children from anticipated backlash.

Feminine role conformity. Compared to the research on masculinity, work on women's role norms and feminine role conformity took much longer to develop beyond the trait perspective. The first multidimensional model was in fact developed for adolescents (Tolman \& Porche, 2000), and even that was limited to only two norms. The Femininity Ideology Scale (FIS; Levant et al., 2007) was not validated or widely available until after the creation of the Conformity to Feminine Norms Inventory (CFNI; Mahalik et al., 2005). Thus, it is unsurprising that there is a greater conceptual divergence between these two measures than in the equivalent male-focused measures. The original CFNI subscales of modesty, invest in appearance, sexual fidelity, and nice in relationships are similar to those of the FIS, although there is no corollary to the FIS' emotionality scale. The CFNI extends beyond the FIS, though, with the subscales of domestic, care for children, and romantic relationship (i.e., investing in romantic relationships). In the revised version of the CFNI, the CFNI-45 (Parent \& Moradi, 2010), 
the nice in relationships subscale is further separated into relationship (i.e., having and maintaining friendships) and sweet and nice (i.e., being nice toward others).

As with masculine role conformity, feminine role conformity is associated with a number of negative health outcomes. Most of the work on correlates of the CFNI and CFNI-45 focuses on clinical outcomes. These include body surveillance and shame, which are in turn predictive of negative eating attitudes, eating disorder symptomology, depressive symptoms, and low self-esteem (Hurt et al., 2007; Mahalik et al., 2005). Similarly, research using the trait perspective of gender has shown that compared to women with stereotypically masculine traits, those with stereotypically feminine traits have increased emotional, somatic, and social symptoms of depression and anxiety (Thornton \& Leo, 1992). Women who not only have feminine traits but also place high importance on traditional feminine roles are at increased risk for substance abuse (Thornton \& Leo, 1992).

Feminine role conformity has also been associated with positive outcomes. Research using the CFNI has found that women's gender role conformity positively predicts their career-related learning experiences in the areas of creativity and personal expression, social interaction, and building things and working outdoors (Tokar et al., 2007). The former two categories have logical associations with feminine norms of expressiveness and success in interpersonal relationships.

Ultimately, outcomes may be dependent upon the particular female role norms under consideration as well as the context. Complex patterns have been found for both alcohol use and sense of purpose (Khairallah et al., 2010; Weller, 2010). Whereas the 
invest in appearance subscale of the CFNI has small but significant positive relationships with alcohol-related problems and the frequency of alcohol use, the nice in relationships, sexual fidelity, and domestic subscales have negative relationships with alcohol use frequency, quantity, and alcohol-related problems (Khairallah et al., 2010). A similarly mixed pattern is observed with women's sense of meaning and purpose in life: whereas modesty is a negative predictor of sense of purpose, the nice in relationships, involvement with children, sexual fidelity, and domestic subscales are all positive predictors (Weller, 2010).

Feminine role conformity also has interpersonal consequences. Relevant outcomes in the areas of romantic relationships and aggression are explored in later sections of this document. For now, it is worth mentioning that most of the relevant research on relationships focuses on women's global adherence to stereotypically feminine traits rather than her fulfillment of specific roles or her engagement in stereotypically feminine behaviors (excepting housework and occasionally childcare). Given the imprecise measurement of gender role conformity, it is difficult to make firm conclusions about backlash for nonconformity in relational contexts.

However, backlash against women's nonconformity has been explored fairly extensively in the context of the workplace, perhaps as a natural outgrowth of the historic "separate spheres" conceptualization of male and female roles. Agentic women are frequently seen as unlikeable, though more capable (Rudman, 1998). Similarly, women who self-enhance are perceived as less likeable and inspire more negative feelings in interactional partners (Powers \& Zuroff, 1988). Interestingly, nonconforming women 
may experience harsher backlash from other women than from men (Rudman, 1998). Theorists suggest that this effect may be due to the perceived threat an agentic or dominant woman poses to other women's sense of a cohesive gender ingroup, although more research is needed. Beyond negative interpersonal evaluations, backlash can also manifest as fewer job offers and promotions, worse outcomes in salary negotiations, undervaluation of accomplishments, and poorer performance evaluations (Heilman, 2001; Janoff-Bulman \& Wade, 1996; Rudman \& Glick, 2001).

Early research on stereotypic gendered characteristics (i.e., using the trait perspective of gender) found that in the absence of accompanying masculine traits, women's self-reported feminine traits negatively predicted career achievement, an outcome that feminine women attributed to having less ability and putting in less effort (Wong, Kettlewell, \& Sproule, 1985). However, after adding educational attainment into the regression equation, only education and masculinity were significant predictors of career achievement. Thus, women's careers may benefit more from having stereotypical masculine traits like independence and assertiveness than they are harmed by having stereotypically feminine traits. Other research suggests that gender nonconformity may benefit women only in specific areas. For example, while women who self-promote rather than self-efface — violating the norms of modesty and deference — are generally seen as less hirable and less attractive as potential interpersonal relationship partners, they are also perceived as more competent (Rudman, 1998).

Why is women's gender nonconformity consistently associated with both positive and negative outcomes? The distinction may lie in the nature of the norms being violated. 
Some traditionally feminine characteristics could be generally maladaptive, such as a lack of assertiveness or an obsessive preoccupation with appearance. To give an example based in research, adolescent girls who behave inauthentically in relationships (i.e., silencing their true thoughts and feelings) to smooth interactions have lower sexual selfefficacy and, in turn, are less likely to use sexual protection (Impett, Schooler, \& Tolman, 2006). In other words, conformity to female role norms such as nurturance and investment in appearance may unfortunately result in unhealthy sexual feelings and behaviors.

Women's gender nonconformity may also be beneficial because of the greater social value placed on traditionally masculine traits (as evidenced by the male role norm of avoidance of femininity). Specifically, some theorists have proposed that because male roles convey status and women's roles do not, a woman can gain status by nonconforming whereas a man only stands to lose status by nonconforming to male gender role norms (Feinman, 1981; Feinman, 1984). The difficulty for women arises in balancing the social capital gained from engaging in masculine roles with the negative assumptions associated with failure to fulfill feminine roles. Especially when masculine and feminine role norms are directly opposed — such as with dominance and deferencewomen's nonconformity may result in a combination of positive and negative outcomes.

The predominant outcome of a given situation might depend on successful impression management; one study found that agentic women who are high in selfmonitoring are able to avoid workplace backlash in the form of denied promotions (O’Neill \& O’Reilly, 2011). As the researchers noted, self-monitoring women "can 
modulate when and how they display the assertiveness, confidence, and aggressiveness necessary to conform to the masculine managerial stereotype, while simultaneously avoiding the backlash effect' (O’Neill \& O’Reilly, 2011, p. 2). Thus, personality factors - at least those relevant to social interactions - can moderate the outcome of women's gender nonconformity.

\section{Gender and Romantic Relationships}

Thus far, gender ideology and gender role conformity have been discussed in general terms and primarily in regards to work relationships or health behaviors/outcomes. However, gender ideology and gender role conformity also influence experiences within romantic relationships. This section begins with a discussion of the research on gender role conformity and relationship behaviors (e.g., conflict, disclosure, support provision). Next, I introduce the relationship concepts of partner-ideals and partner-ideal discrepancies. Then, I discuss how gender and gender ideology predict partner-ideals and interact with partner-ideal discrepancies to influence relationship outcomes. It will become apparent that there is a marked lack of research on gendered partner-ideals. In other words, very little is known about the extent to which people expect and value their romantic partners' gender role conformity, let the alone the impact of discrepancies on "downstream" relationship variables such as relationship satisfaction and conflict.

Before continuing, it is important to note that most of the research in this section was conducted with primarily undergraduate samples. Participants tend to be young, White, and presumably from more privileged socioeconomic backgrounds than the 
general population, although occasionally community members (usually younger adults)

are also included. A small number of studies described, such as those focusing on behavior within existing romantic relationships, utilize more nationally representative samples of couples. However, it is unknown how the overreliance on undergraduate students has impacted results.

Gender role conformity and relationships. The previous section discussed a number of positive and negative outcomes associated with gender role conformity. Gender role conformity also has importance in the context of romantic relationships. Unfortunately, the same measurement issues that plague research on this construct in previously discussed domains are also evident in the research on romantic relationships.

While a handful of newer studies on this topic use the CMNI or CFNI to measure gender role conformity, most research has used the trait perspective and operationalized gender role conformity as being stereotypically "masculine" or "feminine." In the trait perspective, androgynous individuals (i.e., individuals high in both masculinity and femininity) should have the most successful relationships because their behavioral repertoire includes both instrumental and expressive behaviors. Indeed, androgyny has been found to be the best predictor of interpersonal attraction and satisfaction in most studies of short-term interactions (Siavelis \& Lamke, 1992).

However, this pattern does not generalize to long-term romantic relationships. The majority of the research on romantic relationships suggests that femininity of both the self and partner, not androgyny, predicts higher marital satisfaction (Aube \& Koestner, 1995; Frazier \& Esterly, 1990; Lamke, Sollie, Durbin, \& Fitzpatrick, 1994; Siavelis \& 
Lamke, 1992). Trait femininity is linked to a variety of positive relationship maintenance behaviors including the routine use of advice, conflict management, and openness, whereas masculinity predicts strategic use of some of these behaviors (Aylor \& Dainton, 2004). Alternatively, some research has suggested that while women's femininity is important to men's relationship satisfaction, men's androgyny is a better predictor of women's relationship satisfaction (Siavelis \& Lamke, 1992). Femininity in women and androgyny in men is also linked to forgiving partner transgressions (Sidelinger, Frisby, \& McMullen, 2009). Perhaps trait femininity and trait masculinity behave differently: trait femininity could generally contribute to positive relationship behaviors, but trait masculinity might only increase relationship satisfaction among those women who desire it in their partners.

The relational benefits of trait femininity might also be moderated by gender ideology. Aube and Koestner (1995) found that in couples holding traditional gender role attitudes, both husbands and wives are more likely to display gender-stereotypical traits and behaviors. Aligning with their role attitudes, these couples have higher relationship quality if the wife engages in more traditionally feminine behaviors and activities compared to her husband. Puzzlingly, traditional couples experience worse relationship quality if the wife is much more stereotypically feminine than her husband. It is also unclear why this study found wives', but not husbands', relative gender role conformity predictive of relationship quality.

Results obtained using the trait perspective of gender must be interpreted cautiously, particularly when measurement is limited to a single score assessing sex- 
typed personality traits. Recall that research taking a multidimensional approach to gender roles has found that individual roles are tied to distinct outcomes (e.g., Mahalik et al., 2005). The trait perspective not only loses this level of specificity but also excludes some roles entirely. Some researchers have proposed that the association between trait femininity and relationship satisfaction might be more accurately interpreted as the positive impact of expressiveness and nurturance (e.g., Aube \& Koestner, 1995). Regardless, it would be clearly incorrect to assume that individuals' trait masculinity and femininity are equivalent to their fulfillment of the specific gendered norms that have been identified in more recent research. Correlations between trait femininity and the unique dimensions of the CFNI, for example, reveal that older measures of femininity primarily capture relational aspects of the female role (e.g., niceness in relationship, caring for children) to the exclusion of other appearance- and activity-based components (e.g., investment in appearance, domesticity; Mahalik et al., 2005). The dearth of research using more refined measures of feminine role conformity, however, makes it difficult to make firm conclusions about the impact of feminine role conformity on relationship behaviors or satisfaction. It is unknown, therefore, whether all dimensions of traditional femininity contribute to relationship satisfaction in the same way. Some role norms that do not relate directly to interpersonal behaviors, such as thinness or domesticity, might only be beneficial for partners who highly value those norms.

Just as trait femininity overlaps only somewhat with modern measures of role conformity, so trait masculinity correlates moderately with the CMNI (Smiler \& Gelman, 2008). However, research using the CMNI paints a more damning picture of traditional 
masculinity than that of the largely neutral and null results of research on trait masculinity. While instrumentality could be beneficial in certain types of relationships or in particular situations with romantic partners — such as asserting one's needs—certain domains of the masculine role like restrictive emotionality, violence, and primacy of work logically seem to preclude healthy relationship behaviors. In their examination of undergraduate men's conformity to male role norms, Burn and Ward (2005) found that total CMNI scores as well as the dimensions of dominance, playboy, risk-taking, violence, and power over women had negative, small-to-moderate correlations with men's own relationship satisfaction. Of these dimensions, playboy had the strongest correlation. For undergraduate women, however, perceiving their male partners as being high in any CMNI dimension save pursuit of status significantly predicted lower relationship satisfaction. While playboy was also the most influential dimension for women's satisfaction, power over women and dominance were also important. Overall, men's conformity to masculine role norms had a stronger negative association with their partners' relationship satisfaction than with their own. While the dimension of playboy may simply indicate dissatisfaction with monogamous relationships (a relationship characteristic that many people consider non-negotiable), the other dimensions could be problematic for several reasons. Perhaps they preclude the formation of a healthy interdependence, motivate uncaring or abusive behaviors, or create conflict through unfulfilled relationship expectations (e.g., primacy of work may indicate that men's prioritization of work leaves little time or energy for relationship maintenance). 
Holmes (2014) expanded upon prior work using the CMNI by considering the potential mediating effects of relationship behavior. The sample included mostly educated men of a wide range of ages who had been in their romantic relationship for an average of just over eight years. Similar to the results obtained by Burn and Ward (2005), Holmes found that the dimension of playboy had a direct, negative association with men's relationship satisfaction. The respective correlations with playboy, emotional control, and heterosexual self-presentation, however, were all mediated by decreased self-disclosure. In other words, men conforming to any or all of those three norms shared less personal information with their romantic partners, which was in turn associated with low relationship satisfaction. The norm of self-reliance also negatively predicted men's relationship satisfaction, mediated by the conflict resolution tactic of compliance. Thus, men's conformity to traditional gender roles is negatively associated with men's relationship satisfaction both directly and indirectly.

Holmes (2014) also asked women to report on their perceptions of their male partners' relationship behaviors and conformity to male role norms. Perceived conformity to the norms of winning and power over women directly and negatively predicted women's relationship satisfaction. Several relationship behaviors also mediated the impact of specific norms on women's relationship satisfaction. Playboy and emotional control were mediated by decreased self-disclosure; emotional control and winning were mediated by decreased positive problem solving (e.g., use of negotiation and compromise); and dominance, risk-taking, and power over women had a mediated effect through conflict engagement (e.g., using personal attacks in conflict). 
Unexpectedly, risk-taking was associated with less conflict engagement and thus higher relationship satisfaction. Holmes proposed that avoiding the stereotypically masculine use of aggression in conflict could be a form of risk-taking; in other words, perhaps risktaking has a different meaning in the relational context. Generally, however, results suggested that conformity to male role norms has negative implications for women's relationship satisfaction, partially due to maladaptive communication and conflict patterns.

While some might argue that partner perceptions are less accurate than selfreports, it is important to know that perceptions of partner gender role conformity have an impact on the actor's relationship experience. However, past work has failed to examine how gendered partner-ideals could alter the relational impact of perceived partner gender role conformity. If, for example, a woman expected her husband to be aggressive, then his aggressive behavior could have less of a negative impact—or perhaps even a positive impact — on her relationship satisfaction. While this concept has never been tested using measures of gender role conformity, past research has shown that perceptions of the gendered attributes of one's partner are a stronger predictor of relationship satisfaction for both men and women than are self-reports (Lamke et al., 1994; Siavelis \& Lamke, 1992). In other words, while self-reported gender role conformity predicts certain relationship experiences and outcomes, partner perceptions of gender also matter.

Partner-ideals and partner-ideal discrepancies. Gender role conformity can be important for relationship outcomes beyond its association with relationship behaviors. Namely, individuals may expect their partners to conform to particular gender role norms 
and become upset if those expectations are not met. This subsection focuses on both general partner preferences (e.g., wanting someone trustworthy) and those that relate specifically to gender role norms.

Individuals hold expectations for their romantic partners, called partner-ideals. These are the characteristics desired in a potential or current romantic partner. Many partner-ideals are gender-neutral, such as honesty and intelligence. More importantly for the purposes of this project, partner-ideals can also be based in gender role norms and grow from one's gender ideology. For example, a heterosexual woman endorsing traditional masculinity ideology should logically hold components of that ideology as ideals for her romantic partner.

The concept of gendered partner-ideals might remind readers of Deaux and Major's (1987) model of gender-related behavior, first discussed in the literature review section on gender role conformity. Specifically, gender ideology, as part of the gender belief system, influences expectations of interactional partners. However, partner-ideals are primarily aspirational rather than based in descriptive beliefs of gender differences. In other words, partner-ideals represent desires rather than realistic expectations of a partner's behavior. Additionally, expectations may function differently within the context of an established romantic relationship. Deaux and Major (1987) postulate "some elements of gender stereotypes may become unimportant [in long-term relationships]" (p. 373) as individuals possess more individuating information about their partners. However, they also argue, "negotiated role relationships (e.g., a division of labor within the household) may perpetuate and even strengthen other elements of the 
gender belief system" (p. 373). The association between gender ideology and gendered partner-ideals is an empirical question that is partially addressed by the main study described in this manuscript. How these factors function in a given interaction, however, is a question for future research.

To evaluate partners, partner-ideals are compared to "partner-perceptions," or perceptions of a partner's true characteristics. Some have argued that partner-perceptions are less objective or accurate than self-perceptions or third-party observations. Indeed, these three different perspectives should not be assumed to be equivalent; research shows that there is some divergence between an actor's partner-perceptions and the selfperceptions of the partner (Murray, Holmes, \& Griffin, 1996). However, there is also moderate overlap, suggesting that partner-perceptions are based in reality. For the present study, partner-perceptions are the most appropriate measure of partner conformity to gendered norms because those perceptions should logically have the most direct influence on the actor's relationship satisfaction and interpersonal behaviors.

Finally, "ideal discrepancies" describe the degree of mismatch between partnerideals on a particular dimension and partner-perceptions regarding that same dimension. Discrepancies between ideals and actual states (of both the self and of partner characteristics) should generally lead to negative outcomes. This concept of comparison between cognitive representations of ideals and reality draws heavily upon Higgins' (1987) work on discrepancies between the actual, individual ideal, and obligation-based ideal self (named the "actual-," “ideal-," and “ought-self," respectively). In Higgins' Self-discrepancy Theory, differences between these versions of the self are monitored in 
order to regulate the self and maintain or reduce discrepancies. Just as individuals hold expectations and aspirations for their romantic partners, so too they have standards for themselves. Readers may notice that I do not consider a partner-equivalent to Higgins' "ought-self." Past research has inconsistently supported a distinction between the idealself and ought-self (Simpson, Fletcher, \& Campbell, 2001), suggesting that individuals themselves may not clearly distinguish between ideals and obligations. Especially in the realm of relationships, partner-ideals should be based in personal desires and expectations rather than obligations to some unspecified external force.

Currently, the dominant framework for studying partner-ideals is the Ideal Standards Model (ISM; Fletcher, Simpson, Thomas, \& Giles, 1999). In the ISM, there are three overlapping cognitive knowledge structures relevant to relationships. These knowledge structures consist of goals, expectations, and beliefs relating to the self, to one's partner, and to the relationship between oneself and one's partner. The theory states that evaluative categories relating to one's partner and to one's relationship should overlap, as partner-ideals are based on an individual's understanding of the ideal relationship (Fletcher et al., 1999). In other words, someone who aspires to a loving relationship will have corresponding ideal standards regarding a loving romantic partner. Additionally, the ISM proposes that individuals consider ideal-discrepancies when evaluating the quality of partners and relationships, understanding relationship events and characteristics, and regulating their relationships (Fletcher \& Simpson, 2000). Thus, ideal discrepancies should predict relationship satisfaction and behaviors intended to influence one's partner. 
Evolutionary explanations of partner-ideals. Some of the earliest relationship research focused on "mate preferences," an early term for partner-ideals that is still sometimes used interchangeably (e.g., Eastwick \& Finkel, 2008). Studies found consistent sex differences in the traits desired in a romantic partner. These differences center on the theme that females generally value potential mates' resources, whereas males value reproductive capacity (e.g., Buss, 1989). Evolutionary psychologists would explain these diverging preferences through their link to parental investment.

Specifically, because females must make a greater parental investment to successfully pass on their genes (including physically costly gestation and lactation), they should look for mates who are able to provide for and defend offspring. In contrast, the minimum necessary parental investment for a male is much lower (copulation), so he should be motivated to mate with as many fertile females as possible.

The ISM represents an improvement over this narrow focus on resources and reproductive capacity. The specific ideals measured by the ISM were generated from evolutionary theory and solicitation of open-ended partner ideals from undergraduate students (Fletcher et al., 1999). Factor analysis supported three separate dimensions of partner-ideals: Warmth-loyalty (i.e., capacity for intimacy and commitment), Vitalityattractiveness (which includes general health), and Status-resources. The authors explain that each one of the ideals in the ISM provides advantages in human reproduction (Simpson et al., 2001). As previously discussed, vitality-attractiveness should indicate fertility and status-resources should help support the survival of offspring. Warmth- 
loyalty, which represents an addition to usual focus of evolutionary models, should lend itself to devoted partnering and parenting.

However, research using the ISM does not consistently support the sex differences that would be predicted by evolutionary theory. One study using the ISM confirmed women's greater preference for status-resources and men's preference for attractiveness-vitality (Travaglia et al., 2009). A different study, however, found that both men and women considered partner warmth-trustworthiness - essentially a genderneutral ideal—more important than either vitality-attractiveness or status-resources (Fletcher et al., 1999). Two other studies found that women cared more about partners' status-resources and warmth-trustworthiness than did men but that there were no gender differences in the importance of attractiveness-vitality (Campbell et al., 2001; Campbell et al., 2016). The instability of gender differences in partner-ideals calls into question the evolutionary framework on which the ISM was based.

Another major problem with the evolutionarily grounded literature on partnerideals is the focus on prospective preferences. Many of these studies sample individuals who are not currently romantically involved. Rather, they tend to focus on hypothetical attraction and early-stage relationships (most studies using the ISM are exceptions). There are at least two problems with basing conclusions about real relationships from studies using these methods. First, preferences expressed prior to the initiation of a relationship might not accurately represent the partner-ideals that are most important for relationship satisfaction or maintenance. Supporting this idea, at least one study has found that a priori mate preferences only weakly predict real-life romantic interest and 
engagement (Eastwick \& Finkel, 2008). While the expected gender differences in partner-ideals were supported, there were no gender differences in actual romantic interest based on attractiveness and resources after meeting a potential partner. Similarly, one meta-analysis found that the impact of partner physical attractiveness on relationship satisfaction decreases over time after the initiation stage, while perceptions of earning prospects become more important with relationship duration (Eastwick, Luchies, Finkel, \& Hunt, 2013). Thus, individuals may lack the ability to accurately forecast the extent to which they will value or be impacted by specific partner-ideals in a real relationship.

These faulty projections could be potentially based in gendered self-stereotyping. Popular discourse surrounding dating and relationships emphasize women's attractiveness and men's resources (as evidenced, for example, by the prominence of such concepts as "sugar daddies," "trophy wives," and "gold-diggers"), so individuals could adopt these apparently normative preferences in the absence of extensive personal relationship experience. More research on the source of prospective partner-ideals is needed.

The second problem with evolutionarily-based research on partner-ideals is its frequently limited measurement, especially in regards to gendered norms. Since the partner-ideals of attractiveness and resources can usually be trusted to generate sex differences, they are privileged above other gender role norms. While the ISM started with a much broader range of potential partner-ideals than other approaches to partner standards, two of the three final dimensions are attractiveness and resources. Given the findings of Eastwick and Finkel (2008) on the mismatch between prospective and current 
mate preferences, however, it is concerning that the ISM was developed using samples that included single participants and individuals with of an unknown relationship status. Rather than rating partner characteristics based on what they had found important in their real-life relationship experiences, students could have been basing their responses on faulty projection or naïve theories of romantic relationships.

Gender ideology and partner-ideals. While the evolutionary explanation for sex differences in partner-ideals is intuitively appealing, this theoretical viewpoint fails to consider the history and complexity of modern human societies. It is important to consider the social context in which partner-ideals are formed. Like other cognitive representations, standards for partners should be partially learned from others - from both social learning and broader exposure to cultural narratives and values_-and influenced by personal experience.

Eagly and Wood's (1999) Social Role Theory addresses the limitations of the evolutionary perspective. As previously discussed, the theory argues that biological sex differences in size, strength, and reproductive activities resulted in a division of labor. This division of labor resulted in gender roles, which shaped mate preferences within a given society. Thus, while differences in mate preferences can be ultimately traced back to biological differences, they are also influenced by changing social and cultural factors. It follows logically that sex-typed mate preferences should be related to the actual gendered division of labor within a society. Indeed, that is what the data consistently show. At the national-level, gender inequality positively relates to the magnitude of sex differences in mate preferences (e.g., Eagly \& Wood, 1999; Eastwick et al., 2006). In 
other words, to the degree that a society retains the historic division of labor with men engaging in paid work and women responsible for childcare and domestic work, there remain large sex differences in mate preferences.

At the individual level, stereotypic sex-typed mate preferences are negatively predicted by gender egalitarianism (Rempala, Tolman, Odkie, \& Ahn, 2014; Smiler \& Kubotera, 2010). Similarly, women with feminist identities describe their ideal partner as less conforming to traditional male role norms (Backus \& Mahalik, 2011). In particular, feminist women disdain the CMNI dimensions of emotional control, violence, power over women, playboy, and self-reliance. In contrast, nonfeminist women value the norms of emotional control, power over women, dominance, self-reliance, and disdain for homosexuals but dislike risk-taking. Thus, even among women endorsing traditional masculinity ideology, some male role norms are more desirable than others.

Interestingly, gender ideology predicts different preferences depending on the kind of relationship being considered. Delacollette, Dumont, Sarlet, and Dardenne (2013) found that men are more likely to prescribe warmth to a woman in a family context than in a professional one. This effect was mediated by the perceived personal benefit of the woman's warmth, suggesting that men may hold different standards for women in particular roles because they expect to benefit more from the woman's conformity. Other research has shown that these shifting expectations depend on the man's gender ideology. Whereas men with traditional role attitudes judged women's instrumental traits as more desirable in a work context and expressive traits more desirable in a romantic context, egalitarian men made no such distinction (Smiler \& 
Kubotera, 2010). The fact that gender ideology can lead to expectations that vary based on the type of relationship and the context in which the evaluations are being made underscores the importance of conducting research on gender ideology and gender role conformity in the context of romantic relationships. Specifically, gender ideology could function differently in existing romantic relationships than in the professional or hypothetical relationships usually studied.

Like gender ideology, sexism also predicts partner-ideals. Sexism is more complex than simply holding negative stereotypes or attitudes. Glick and Fiske (1996) argued that sexism actually represents ambivalent attitudes toward women; in addition to the hostile attitudes ("hostile sexism") characteristic of other forms of prejudice, people may also harbor seemingly positive, yet nevertheless harmful, attitudes toward women. This "benevolent sexism" involves attitudes that "are subjectively positive in feeling tone (for the perceiver) and also tend to elicit behaviors typically categorized as prosocial (e.g., helping) or intimacy-seeking (e.g., self-disclosure)" (Glick \& Fiske, 1996, p. 491). Benevolent sexism is comprised of three distinct attitudinal domains: protective paternalism (women should be protected and cherished), gender differentiation (men and women have innately complementary roles), and heterosexuality (valuing heterosexual romantic relationships). "Ambivalent sexism" involves simultaneous endorsement of both hostile and benevolent sexism, a pattern observed in many samples.

Ambivalent sexism should shape reactions to feminine role (non)conformity. Hostile sexism should predict negative reactions toward gender nonconforming women (primarily because of the threat they pose to men's dominance), while benevolent sexism 
should predict positive reactions toward women's gender conformity (which maintains the status quo of gendered relations). There are clear conceptual overlaps between ambivalent sexism and measures of femininity ideology. For example, a hostile sexist would expect women to exhibit deference and sexual purity, and a benevolent sexist would laud what they would view as women's natural affinity for caretaking. Perhaps because of this similarity, it is not uncommon for researchers to treat sexism and gender ideology as interchangeable. However, as discussed in the beginning of this document, sexism is frequently grounded in both descriptive and prescriptive norms, whereas ideology focuses on prescriptive norms.

While less commonly studied, attitudes toward men can also be categorized as ambivalent. Glick and Fiske (1999) found evidence that, similar to ambivalent sexism, many people hold both benevolent and hostile attitudes toward men relating to aspects of maternalism, gender differentiation, and heterosexuality. While hostility toward men is theoretically rooted in resentment of men's higher status in society, benevolent attitudes are driven by admiration. Ambivalent attitudes toward men are positively correlated with ambivalent sexism in both men and women, suggesting that both categories of attitudes could be based on an underlying, traditional gender ideology.

Research has suggested that to the extent individuals endorse ambivalent sexism and ambivalent attitudes toward men, they will also have sex-typed mate preferences (Eastwick et al., 2006). In other words, having stereotypical gender role beliefs and attitudes predicts preferences for gender-conforming romantic partners. For example, a woman's hostile and benevolent attitudes toward men predict her preference for a mate 
with good financial prospects (Eastwick et al., 2006). However, there is some conflicting research about which particular attitudes (i.e., hostile or benevolent) predict traditional mate preferences in each gender. One study suggested that only benevolent sexism and benevolent attitudes toward men, respectively, predict preferences for partners pursuing traditional gender roles (Thomae \& Houston, 2016). Another found that gendered partner-ideals were related to benevolent sexism in both genders but to hostile sexism only in men (Lee, Fiske, Glick, \& Chen, 2010). Similarly, preferences for traditional dating behaviors - such as a man paying for a date and a woman taking her husband's surname in marriage - are predicted by ambivalent sexism in both genders, although women's hostile sexism is only weakly related (Paynter \& Leaper, 2016). Still other studies have found that traditional mate preferences are predicted primarily by women's benevolent sexism and by men's hostile sexism (Sibley \& Overall, 2011; Travaglia, Overall, \& Sibley, 2009).

The distinction might lie in the specific gender stereotype under consideration. As previously discussed, the evolutionary perspective tends to focus on men's status or financial security and women's physical attractiveness (e.g., Rempala et al., 2014; Sibley \& Overall, 2011), whereas others examine traits such as dominance and warmth or even specific role expectations (e.g., Lee et al., 2010; Thomae \& Houston, 2016). Readers will recall that in the previous subsection, Travaglia et al. (2009) found confirmation of the usual gender differences in mate preferences (i.e., women placed more importance on status/resources while men placed more on attractiveness/vitality). However, these differences were partially explained by endorsement of ambivalently sexist beliefs. 
Women endorsing benevolent sexism primarily valued potential partners' ability to fulfill protector and provider roles, whereas men endorsing hostile sexism highly valued partners' physical attractiveness. For both genders, benevolent sexism positively predicted while hostile sexism negatively predicted the partner-ideal of warmth/trustworthiness. This study demonstrates the importance of ideological factors for partner-ideals. However, measurement was limited to the three categories of partnerideals contained in the ISM, two of which were relevant to gender roles. As emphasized by Mahalik et al. (2005), it is important to consider a variety of gender role norms, as each norm may be tied to distinct outcomes.

While the scope of the ideals examined in work on partner-ideals is usually limited, at least one study has expanded it to include other traditionally gendered characteristics. Drawing upon work in ambivalent sexism and ambivalent attitudes toward men, Lee et al. (2010) asked undergraduates in the United States and China to generate prescriptive and proscriptive ideals for an other-sex romantic partner. Exploratory factor analysis revealed that American men desired partners who were warm, traditionally feminine, attractive, and strong, while they rejected abusive, overly feminine, unattractive, and nontraditional partners. American women wanted warm, romantic, attractive, strong, and traditionally masculine partners and disliked rejecting, feminine/liberal, abusive, jealous/superficial, clingy, and traditionally masculine partners. The presence of contradictory ideals (e.g., being traditionally masculine was both prescribed and proscribed) suggests that there is substantial variation in partner-ideals even within genders (however, because participants were not asked to confirm their 
sexual orientation, there is a chance that certain freely-generated ideals were intended to describe someone of the same gender). Among Americans of both genders, benevolent sexism predicted partner-ideals corresponding to traditional gender roles. Thus, individual attitudes regarding gender appear to influence a variety of gendered partnerideals beyond the well-studied dimensions of resources and attractiveness.

Gender ideology and relationship behaviors. Before gender ideology was considered as a contributor to partner-ideals, it was studied in regards to relationship behaviors. Because most researchers interested in gender ideology held feminist attitudes, studies frequently focused on relationship outcomes that demonstrated substantial gender imbalances and were hypothesized to contribute to the maintenance of societal gender inequality: household labor, caretaking (especially childcare), and paid labor outside the home. While studies have generally focused on behavioral outcomes and global gender ideology rather than partner-ideals, the pattern of results are congruent with relationship outcomes such as relationship satisfaction being impacted by the degree of congruency between relationship- or partner-ideals and perceived reality.

If people with traditional gender ideologies have more sex-typed partner-ideals, then logically they should also desire more traditional roles within a romantic relationship (Peplau, Hill, and Rubin, 1993). However, in reality, the relationship between gender ideology and behavioral conformity to gender roles is complicated. Gender ideology is more strongly predictive of some aspects of domestic work, such as husbands' time spent providing childcare, than others (Kluwer \& Mikula, 2003). Despite the fact that women are generally more egalitarian than men, women nevertheless perform the majority of the 
household labor (Greenstein, 1996; Lavee \& Katz, 2002). Women's gender ideology is not a strong predictor of their engagement in household labor. Instead, the division of household labor is determined by the interaction of partners' gender ideologies in a way that reflects men's higher status. Specifically, women's fulfillment of the traditional household role is dependent upon their male partners' gender ideologies. Unless both partners have progressive gender ideologies, the woman usually ends up bearing the brunt of the domestic labor regardless of her own preferences (e.g., Greenstein, 1996). In other words, women retain responsibility for fulfilling the traditional domestic role unless their nonconformity is also supported by their male partners.

Women's gender ideology does, however, relate to the perceived unfairness of the traditional household division of labor. Specifically, egalitarian women are more likely than traditional women to perceive an unequal division of household labor as unfair (Lavee \& Katz, 2002). Similarly, an unequal division of labor predicts decreased marital satisfaction for egalitarian but not for traditional women. In other words, traditional women may be unaffected by an unfair burden if it matches their role expectations. Other research has confirmed that a sense of fairness, rather than the actual balance of the division of labor, is a better predictor of marital satisfaction (Greenstein, 1996; Shelton \& John, 1996). Thus, the impact of gender role conformity in the area of household labor on marital satisfaction is mediated by the degree to which behaviors match ideals.

Traditional gender roles also dictate that men provide for their families monetarily. What happens, then, when women fill the breadwinner role? Coughlin and Wade (2012) examined masculinity ideology and relationship quality among men whose female 
partners earned comparatively more money. In the context of this gender role violation, men's traditional masculinity ideology predicted lower relationship quality. Conversely, a flexible conceptualization of masculinity positively predicted relationship quality. This effect was mediated by the degree to which the income disparity was personally important, a variable that was itself positively related to traditional masculine ideology (likely because men with traditional masculinity ideology would perceive income disparity as a personal failure to fill the proper breadwinner role). Thus, with both domestic and paid labor, whether outcomes are positive or negative is determined by the match between gender ideology and the extent to which partners are fulfilling traditional gender roles. That is to say, gender ideology establishes (or perhaps reflects) the standards to which people hold their romantic relationships.

Other research suggests that beyond its interaction with traditional roles, masculinity ideology is harmful to relationships through its impact on relationship behaviors. McGraw (2001) found that men endorsing, or perceived by their partners as endorsing, traditional masculinity ideology are more likely to display destructive relationship patterns (e.g., less closeness and caregiving, more anger and emotional reactivity). Unsurprisingly, then, men's traditional masculinity ideology negatively predicts both men's and women's relationship satisfaction. Wade and Donis (2007) extended this line of inquiry to include homosexual men and found that, among both heterosexual and homosexual men, traditional masculinity ideology predicts both lower psychological relatedness and lower relationship satisfaction. 
One factor to consider is the interaction of romantic partners' respective gender ideologies. Generally, studies have shown that people prefer similar others as both platonic and romantic relationship partners. Thus, it is not surprising that romantic partners tend to have at least moderately similar gender ideologies, egalitarian attitudes, and feminist identities (Aube \& Koestner, 1995; McGraw, 2001; Peplau et al., 1993; Rudman \& Phelan, 2007), an effect that is not explained by attitudinal convergence over time (Aube \& Koestner, 1995). Several studies have shown that couples' similarity in endorsement of egalitarianism positively predicts relationship satisfaction (Aube \& Koestner, 1995; Lye \& Biblarz, 1993; McGraw, 2001). Phrased another way, couples with dissimilar gender role attitudes tend to be less happy with their relationship. Some researchers have speculated that dissimilarity harms relationship satisfaction largely through increased conflict (e.g., Amato \& Booth, 1995; Faulkner, Davey, \& Davey, 2005). Especially in regards to household labor, egalitarian women face the uphill battle of challenging the status quo (Greenstein, 1996). On the flip side, women with feminist partners report having healthier relationships (e.g., low levels of conflict, high levels of intimacy and positive shared experiences; Rudman \& Phelan, 2007). This last study also found that men with feminist partners have greater sexual satisfaction and perceptions of relationship stability than men with nonfeminist partners. Relationship equality positively predicted both men's and women's perceptions of relationship quality, again possibly because of decreased conflict over the appropriate roles for each partner.

As with gender ideology, hostile and benevolent sexism are linked to experiences in romantic relationships. In their extensive body of work on sexism within romantic 
relationships, Hammond and Overall (2013a, 2013b, 2014, 2015, 2016) argue that while benevolent sexism relates to generally positive, romanticized views of relationships, hostile sexism predicts negative, adversarial views. Because men's hostile sexism is largely based in perceived challenges to male power, hostilely sexist men could view romantic relationships as a power struggle in which female partners aim to exploit their dependence. Given this mindset, it is unsurprising that men—but not women—high in hostile sexism tend to engage in more negative communication strategies, feel more manipulated by their partners, and perceive their partners are using more negative strategies than the partners themselves report using (Hammond \& Overall, 2013a). Regardless of accuracy, perceiving one's partner as using negative communication strategies predicts lower relationship satisfaction, demonstrating the importance of perception.

While men's hostile sexism is linked to negative relationship behaviors and benevolent sexism with positive behaviors (Overall, Sibley, \& Tan, 2011), benevolent sexism is not necessarily beneficial to romantic relationships. In fact, it predicts lower relationship satisfaction (Casad, Salazar, \& Macina, 2014). Because benevolent sexism implies a romanticized view of relationships, it may foster unrealistically idealized expectations for relationships. These unrealistic expectations include viewing any disagreement as inherently destructive, seeing the relationship as destined, and expecting partners to be able to read one another's minds (Hammond \& Overall, 2013b). Thus, holding these expectations creates room for disappointment. Hammond and Overall (2013b) discovered that benevolently sexist women are especially sensitive to 
relationship problems and their partners' negative behavior, likely because it conflicts with these relational expectations. Individuals of both genders are more likely to dissolve a relationship in which a partner does not meet their standards if they are high in benevolent sexism (Hammond \& Overall, 2014), showing that benevolent sexism may paradoxically decrease relationship stability. Once again, the impact of a partner's gendered behavior on relationship satisfaction depends upon one's expectations.

Partner-ideal discrepancies and relationship satisfaction. While many of the aforementioned studies used gender ideology or sexism as a proxy for relationship or partner ideals, others have measured partner-ideals and partner-ideal discrepancies directly. According to the ISM, ideal discrepancies in established relationships should result in low relationship satisfaction. Research has largely supported this conclusion (e.g., Campbell, Overall, Rubin, \& Lackenbauer, 2016; Campbell, Simpson, Kashy, \& Fletcher, 2001; Fletcher et al., 1999; Fletcher, Simpson, \& Thomas, 2000; Jaspaert \& Vervaeke, 2014). Studies using the ISM have frequently found that ideal discrepancies on all three dimensions predict lowered relationship satisfaction (Campbell et al., 2001; Campbell et al., 2016; Fletcher et al., 2000). However, at least one study utilizing a different model of ideal-standards than the ISM has suggested that some ideals are more important for relationship satisfaction than others. Stephanou (2012) found that only ideal discrepancies in trustworthiness (and not in the dimensions of warmth-intimacy and attractiveness-vitality) predicted the extent to which Greek young adults viewed their romantic relationship positively. 
Given the strength of the evidence that ideal discrepancies predict relationship satisfaction, it is unsurprising that ideal discrepancies also predict relationship dissolution. Eastwick and Neff (2012) found that partner-ideal discrepancies positively predict divorce three and a half years after marriage. Similarly, Fletcher et al. (2000) found that ideal discrepancies predict dissolution during the first three months of dating relationships. Importantly, this relationship was mediated by the deleterious effect of ideal discrepancies on relationship satisfaction.

Because of the well-documented existence of positive illusions in romantic relationships (e.g., Fletcher \& Kerr, 2010), it is important to determine whether the association between ideal-discrepancies and relationship satisfaction matches the direction of causality theorized by the ISM or whether it is a result of unrealistically positive partner-perceptions in happy relationships. Longitudinal work on the ISM has found that while having only small partner-ideal discrepancies predicts increases in satisfaction with one's relationship and partner over time, the reverse is not true (Campbell et al., 2001; Fletcher, Simpson, \& Thomas, 1998, as cited in Simpson et al., 2001). In other words, ideal discrepancies most likely impact relationship satisfaction; the association between the two factors cannot be explained by positive illusions generated by high relationship satisfaction.

The ISM states that large ideal-discrepancies should not only decrease relationship satisfaction, but should also motivate individuals to take action to decrease the gap between ideals and partner-perceptions. As implied in the last paragraph, one strategy to decrease ideal-discrepancies is to develop an overly optimistic view of one's 
partner (i.e., changing perceptions to more closely align with existing ideals).

Alternatively, one could shift one's ideals to match partner-perceptions.

However, strategies are not limited to cognitive shifts; individuals can also attempt to decrease partner-ideals by regulating (i.e., changing) the partner (Fletcher et al., 1999; Overall, Fletcher, \& Simpson, 2006). Regulation can take many forms ranging from gentle encouragement to violence. For example, a woman whose male partner does not live up to her ideals in regards to status-resources might suggest that he ask for a promotion, help him identify better jobs with current openings, taunt him in an attempt to shame him into changing, control access to joint resources as a form of punishment, or take any number of other approaches to achieve the same goal. Regulation attempts do not necessarily predict improved relationship satisfaction; not only do they increase the salience of ideal discrepancies, but they can also create conflict. Of course, there is also no guarantee that they will be successful. Studying this topic, Overall et al. (2006) confirmed that ideal-discrepancies lead to more regulation attempts, although regulation attempts are detrimental to relationship satisfaction. Counter to the theory underlying the ISM, regulation attempts predict future increases in ideal discrepancies. This finding might be explained by the fact that most regulation attempts are perceived as unsuccessful (Overall et al., 2006). Individuals whose regulation attempts fail could see their partners' shortcomings as more severe or enduring than previously assumed.

\section{Intimate partner violence}

IPV represents one potential regulation strategy that individuals could use to attempt to decrease partner-ideal discrepancies. While the term "strategy" has sometimes 
been used in the IPV literature to connote that an individual is engaged in an intentional, ongoing pattern of control (e.g., Johnson, 2006), it is not intended to convey premeditation in this manuscript. Rather, I conceptualize IPV as a potential behavioral technique used in reaction to perceived partner-ideal discrepancies with the goal of changing the partner's behavior to more closely align with partner-ideals. In some cases this behavior might be part of a pattern of control, but it is not necessarily. Also important is the possibility that the goal of regulating one's partner to align with partnerideals is implicit; perpetrators of IPV might perceive their actions as a result of situational anger, disappointment, or another negative emotion without identifying the cause of that emotion as a partner-ideal discrepancy.

The Centers for Disease Control and Prevention (2016) defines IPV as "physical, sexual, or psychological harm by a current or former partner or spouse" (p. 1). This harm usually involves intent to cause injury, suffering, or humiliation or to otherwise exert control over one's partner. For the purposes of this study, "partner" includes current dating partners, intimate cohabitants, and spouses. The terms "domestic violence," “domestic abuse," "spousal abuse," "wife abuse," and "battering" are commonly used interchangeably with "IPV" (Ali \& Naylor, 2013). However, the term "IPV" is used in this manuscript because it connotes that violence can happen between any intimate partners (i.e., it does not exist only in marital relationships), avoids assuming that the target is female, and does not include the implication that violence is a domestic issuemeaning that it is limited to behaviors within the home or should be a private family matter. 
While IPV is now recognized as an issue of societal and legal importance, it remains a widespread problem. Based on the nationally representative 2011 National Intimate Partner and Sexual Violence Survey, lifetime prevalence of experiencing physical IPV is about $32 \%$ among women and $28 \%$ among men (Breiding et al., 2014). (For severe violence, rates were about $22 \%$ for women and $14 \%$ for men.) Within the past year, about $4 \%$ of women and $5 \%$ of men experienced physical IPV. Regarding psychological violence, lifetime prevalence rates were roughly $47 \%$ for both genders, whereas rates within the past year were about $14 \%$ for women and $18 \%$ for men. Lifetime prevalence of rape and sexual coercion by intimate partners were also high: $45 \%$ and $75 \%$, respectively, for women, and $29 \%$ and $70 \%$, respectively, for men. Based on these rates, the majority of individuals within the United States will experience sexual coercion by an intimate partner at some point in their lives, nearly half will experience psychological aggression, and slightly less than one third will experience physical violence. If we hope to develop effective strategies to counteract this widespread public health problem, we must first understand its etiology. This proposal considers both gender role conformity and partner-ideal discrepancies as contributors to IPV. Gender role conformity — particularly masculine conformity—has frequently been studied as a predictor, although many of these studies suffer from serious measurement limitations. Only one study to date has considered the role of partner-ideal discrepancies in IPV. Before continuing, it is important to note that most of the psychological research on IPV focuses on physical violence rather than other forms of abuse, possibly because measurement of physically violent behavior is believed to be more objective than 
psychological or sexual aggression (accuracy and partner agreement in reporting is discussed in a later subsection), or perhaps simply because the impact of physical violence is more visible. This restriction is a limitation of this area of research. Unless otherwise specified, the studies and perspectives described in this section refer to physical violence.

Theoretical perspectives of IPV. There is little agreement regarding the causeor causes—of IPV. Everything from genetics and brain injuries to situational and relational factors has been proposed as a potential contributing factor. In their review article, Ali and Naylor (2013a) categorized the major theoretical perspectives as biological, psychological, feminist, sociological, and nested ecological. A full review of all major perspectives on IPV is beyond the scope of the current project (see Ali \& Naylor, 2013a; 2013b for a more complete analysis). Additionally, the distinction between perspectives is not always clear-cut; researchers may build upon and borrow from multiple frameworks. For example, many psychologists studying IPV measure variables implicated by feminist perspectives. Thus, the present discussion of theoretical backgrounds will focus on the perspectives with the most relevance to the current project: feminist and psychological. Findings reported in this section are interpreted in regards to their potential implications for partner ideal-discrepancies.

Feminist perspectives. Because the feminist movement is primarily responsible for bringing the issue of IPV — and violence against women more broadly—into the public consciousness, the feminist perspective of IPV is one of the most dominant. Traditionally, these perspectives focus on IPV as a reflection of - and as a tool used to 
maintain — men's greater power in patriarchal societies (Ali \& Naylor, 2013b). Within patriarchal societies, IPV is seen as an acceptable way for men to exert their natural or proper dominance. Thus, elements of the larger social environment, rather than individual or situational factors, are believed to be the main cause of IPV. To reduce IPV, women's social status and power must increase.

Power and control are central issues in feminist perspectives of IPV (Ali \& Naylor, 2013b; Dobash \& Dobash, 1979). Because of men's historically greater physical, social, and legal power, they were able to use IPV to strategically leverage their power within romantic relationships without fear of negative external repercussions. Within this perspective, IPV perpetration is believed to be triggered by perceived threats to one's social or relational power (Anderson, 1997; Goode, 1971; Hotaling \& Sugarman, 1986). Importantly, many feminist perspectives view all forms of relational violence as underpinned by a desire to exert male power or regain dominance (Ali \& Naylor, 2013b).

Many researchers have criticized feminist perspectives of IPV, arguing that because women perpetrate as much or more IPV than men (see Dobash and Dobash [2004] for a discussion of the disputes surrounding this point), IPV cannot be caused by patriarchal systems and cultures. In other words, feminist perspectives are incorrect because they cannot explain bidirectional and female-perpetrated IPV; true femaleinitiated violence would violate the gendered power dynamics on which these perspectives are based. However, many feminist theorists maintain that most femaleperpetrated violence is actually defensive or reactive to men's violence. 
Recently, some psychologists have attempted to reconcile feminist ideologies with the evidence supporting the existence and prevalence of female-perpetrated IPV. They argue that examining IPV from a relational perspective, rather than only through the lens of patriarchal power dynamics, is not at odds with feminist values (George \& Stith, 2014). The traditional feminist perspectives oversimplify power and identity, treating each gender as a monolith. Instead, it is important to consider how the experience of and contributors to IPV may vary intersectionally.

Another critique of the feminist perspectives, exemplified by Dutton (1994), is that by focusing on social structure (i.e., patriarchy) and social categories (i.e., gender), feminist research neglects between-person factors that may be stronger and thus ultimately more useful predictors of violence perpetration. If IPV is a direct result of the patriarchy and gendered power imbalances, then IPV perpetration should be perfectly determined by the presence of those factors. Of course, the reality is much more complex. The fact that most men - even those belonging to patriarchal cultures — are not violent points to the important role of lower-level factors (Kilmartin \& McDermott, 2016).

Psychological perspective. While not exclusive with feminist perspectives, psychological examinations of IPV generally focus on individual difference factors, and sometimes on relational factors, linked to perpetration or victimization. Popular variables include psychopathology, personality differences, attachment styles, anger and hostility, self-esteem, communication skills, and substance abuse (Ali \& Naylor, 2013a). While some researchers focus on more proximal predictors (e.g., anger or hostility) that present promising opportunities for intervention in existing abusers, others attempt to identify 
developmental risk factors (e.g., witnessing childhood abuse and the development of insecure attachment styles) that could lead to preventative efforts.

For the present discussion, the most important category of psychological variables related to IPV involves interpersonal communication and other relational factors. In a meta-analysis by Capaldi et al. (2012), the best-established relational risk factors for IPV were marital status (generally, single and married women are less vulnerable to IPV victimization than are women of other relationship statuses), low relationship satisfaction (which is discussed in a later section), negative emotionality and jealousy, and relational conflict. All of the aforementioned risk factors for IPV, excepting relationship status, could have relevance to gendered partner-ideals and partner-ideal discrepancies. Based on the Ideal Standards Model, ideal discrepancies should broadly decrease relationship satisfaction and increase negative emotionality. Ideal discrepancies in specific domains, such as purity for women, seem especially likely to increase jealousy.

However, the presence of ideal discrepancies alone is clearly not sufficient to cause severe violence. Most couples seem to handle these and other relationship problems without resorting to physical violence, as is evidenced by estimates that less than one third of young adults in romantic relationships had either perpetrated or been the victim of physical assault in the past year (Straus \& Douglas, 2004). However, it is a possibility that individuals primarily use non-physical IPV, rather than physical IPV, to attempt to decrease ideal discrepancies. While Straus and Douglas (2004) found that the majority of individuals were not in physically abusive relationships, over three-quarters of the sample experienced psychological aggression (with similar rates for both 
perpetration and victimization). It is also unknown how many couples use control behaviors in the absence of physical IPV; usually, control behaviors are only considered in regards to how they change the dynamics of physical violence (see the discussion of IPV typologies below).

Psychological examinations of IPV have identified individual factors that increase the risk of physical IPV in the face of dissatisfaction or conflict. For example, past research has found that abusive men tend to have worse communication and social skills, are less assertive in interactions with their spouses, and are less competent problemsolvers (Holtzworth-Munroe et al., 1997). Lacking these skills could increase the likelihood of turning to violence when faced with a relational problem.

A major strength to the psychological approach to IPV, particularly in contrast to the original feminist perspectives, is the idea that IPV could be motivated or caused by different things in different people. This stands in contrast to the idea that all IPV is motivated by the desire to gain power and control. The recognition of this potential diversity creates the opportunity not only for a better understanding of the causes of IPV in individual relationships, but also for the development of more targeted interventions.

Several researchers have proposed typologies to represent these different patterns of IPV. A full review of these typologies is beyond the scope of this manuscript (for a more complete discussion, see Ali, Dhingra, \& McGarry, 2016). However, it is worth noting that the most influential of these, Johnson's (1995) typology, recognizes that violence can be perpetrated by either or both members of a romantic couple and categorizes violence based on the presence or absence of a pattern of coercive control. 
“Coercive controlling violence" describes ongoing control and manipulation of a romantic partner, and it is most commonly perpetrated by men (Graham-Kevan \& Archer, 2003; Johnson, 2006). In contrast, "situational couple violence" does not include an ongoing pattern of control; instead, violence occurs when individuals fail to maintain control of themselves in situations of conflict. Situational couple violence is the most common type of IPV in the general population and is perpetrated by similar numbers of men and women (Ali et al., 2016; Johnson, 2006). For the sake of completeness, I will note that later updates to Johnson's (1995) typology included the addition of mutually violent control, violent resistance (i.e., violence used in response to victimization), and violence triggered by the dissolution of a romantic relationship (Johnson, 2006).

Before continuing, it is important to address a major criticism of psychological approaches to the study of IPV: some approaches are interpreted as blaming victims or absolving perpetrators of personal responsibility (e.g., George \& Stith, 2014; Johnson, 2011). For example, anger is a consistent risk factor for IPV perpetration. Anger management, however, is a highly contentious response to IPV. Many see it as diffusing responsibility, implying provocation (the victim must have done something to anger the perpetrator), and failing to grasp the larger social problems contributing to violence (George \& Stith, 2004). Some critics have also (rightfully) pointed out that because many abusive men are only violent within the context of their romantic relationship, their problem cannot be one of anger management; they are able to manage their anger successfully when it benefits them. However, for a person whose violent behavior is impulsive and triggered by specific conflicts rather than an ongoing desire to control 
one's partner (as in Johnson's [1995] situational couple violence), anger management seems potentially useful.

Ultimately, few psychologists would argue that there is one single root cause of all forms of IPV. The fact that even the strongest predictors of IPV (e.g., marital satisfaction, attitudes supportive of IPV, substance use) are far from perfect supports this conclusion (Stith, Smith, Penn, Ward, \& Tritt, 2004). Thus, different people may require different kinds of intervention to cease their violent behavior. Condemning anger management, family therapy, or other psychologically-based approaches across the board, then, may be throwing the baby out with the bathwater. A theory or approach is not necessarily worthless because it cannot explain or address all cases of IPV.

As we have seen in this section, IPV is a complex phenomenon influenced by a variety of factors at multiple levels (Bowen, 2011). The main study described in this document does not attempt to develop a unified explanation for IPV. Rather, it identifies a new potential, relational contributor to IPV: gendered partner-ideal discrepancies of romantic partners. As previously discussed, IPV is only one of many possible reactions to ideal discrepancies. It is likely that most people, in most situations, would respond to ideal discrepancies non-violently. However, knowing that gendered ideal discrepancies are positively associated with IPV would present new possibilities for intervention. Given that gendered partner-ideals seem to be based in gender ideology, one such potential area for intervention involves gender ideology.

IPV and gender ideology. One of the major predictions of the feminist perspectives of IPV is that gendered power imbalances, and the traditional gender 
ideology reflecting that imbalance, should predict IPV. As noted by Smith (1990), patriarchy can be considered on two levels. At the societal level, men have structural power over women. At the familial level, men have power and dominance within the family (particularly in romantic relationships). Traditional gender ideology legitimizes imbalanced gender dynamics at both of these levels.

The feminist perspective of IPV has been largely supported at both the societal and individual levels of analysis. Across several nations, traditional gender ideology and hostile sexism both positively predict women's victimization (Archer, 2006). However, progressive gender ideology is not just predictive of less violence toward women; it also relates to the gender balance of violence victimization. Specifically, the gender balance of partner violence covaries with national levels of gender equality such that fewer women relative to men are victimized in countries with greater gender equality (Archer, 2006). In the United States, gender equality at the state level is inversely related to women's victimization (Straus, 1994). Thus, IPV—and specifically female victimization — is related to gender equality and gender ideology at several higher levels of analysis.

While a few studies have examined state- or national-level predictors of IPV prevalence, the majority of psychological research on IPV and gender ideology has focused on individual-level gender ideology, egalitarianism, or sexism. If it is true that IPV is motivated by a belief in male dominance, then men should be more likely to perpetrate IPV to the extent that they believe in and seek to maintain their natural dominant status in romantic relationships. The complementary hypothesis for women is 
that traditional gender ideology should relate to victimization through acceptance of male partners' dominance and of women's deference.

Early research on these predictions had mixed results. One review of the risk factors in male-to-female IPV found that wives' traditional gender ideology was positively associated with IPV in about half of previous studies, while husbands' traditional gender ideology was unrelated to IPV more often than not (Hotaling \& Sugarman, 1986). A newer meta-analysis, however, examined seven studies with a total of almost twelve hundred participants and found that having non-egalitarian attitudes was related to men's physical IPV perpetration at $r=.29$ (Stith et al., 2004). The effect size was of about the same magnitude as marital satisfaction and attitudes condoning violence, two well-supported risk factors for IPV. Thus, individual, traditional gender ideology predicts men's IPV perpetration.

More recently, researchers have begun considering the potentially interactive effects of romantic partners' respective gender ideologies on IPV. In other words, rather than traditional gender ideology having main effects on men's IPV perpetration and women's IPV victimization, certain combinations of gender ideologies could be more likely to result in IPV. For example, having incongruent gender ideologies could increase the likelihood of conflict over spouses' proper and expected roles. Women's progressiveness has the added component of an implied threat to traditional husbands' preferred patriarchal power balance. DeMaris, Benson, Fox, Hill, and Wyk (2003) conducted the first study on this idea using data from married couples participating in the National Survey of Families and Households. In couples with congruent gender 
ideologies (i.e., both traditional or both progressive), gender ideology was not significantly related to male-perpetrated IPV. Similarly, there was no increased risk among progressive husbands married to traditional wives. However, traditional husbands married to progressive wives were significantly more likely to perpetrate IPV. Compared to progressive couples, couples in which only the woman was progressive had $62 \%$ higher odds of intense male-to-female IPV. These results suggest that the inconsistent support for the relationship between gender ideology and IPV in past research might be explained by the failure to examine interactive effects between romantic partners.

A few years after DeMaris et al.'s (2003) study, Atkinson, Greenstein, and Lang (2005) tested a related idea using the same dataset. Earlier, theorists had proposed that violence is a tool used to gain power in relationships (Goode, 1971), particularly by men with fewer social and material resources than their female partners (e.g., Anderson, 1997). Atkinson et al. (2005) built upon this "relative resource theory" to incorporate the contextualizing variable of gender ideology. Their "gendered relative resource theory" explained that the outcome of a man's relative resources depends upon his gender ideology. Namely, men with traditional gender ideologies should perceive a relative lack of individual resources as a threat to their familial power or as evidence of their failure to fulfill the breadwinner role. Traditional men with relatively greater resources and progressive men of any resource status should not perceive such a threat. Thus, only traditional men should respond to having relatively few resources with violence. Results supported this hypothesis. Husbands' relative lack of resources (measured as the 
proportion of husbands' to wives' earnings) predicted their perpetration of IPV, but only among husbands with traditional gender ideologies.

While women's gender ideology was not considered in Atkinson et al. (2005), the study nevertheless builds upon past research by suggesting a potential mechanism by which men's traditional gender ideology results in IPV. Specifically, some maleperpetrated IPV could be in reaction to a mismatch between gender role expectations (e.g., men's fulfillment of the breadwinner role) and reality. Such a mismatch is most likely to occur in couples with partners endorsing different levels of gender role traditionalism, which could explain the interactive effects found in DeMaris et al. (2003). For example, a progressive woman would be less likely than a traditional woman to conform to feminine role norms. If that progressive woman is partnered with a progressive man, then he will not expect her to fulfill those norms and will thus be unaffected by the degree of her gender role conformity. However, if her partner holds a traditional ideology, her nonconformity becomes a potential contributor to his dissatisfaction.

It is important to note that in Atkinson et al.'s (2005) study, the exact mechanism of IPV related to interactive gender ideologies is unclear. Are men with traditional gender ideologies using violence to regain relational power based on their own failure to fulfill traditional gender roles, or are they responding their partners' nonconformity to the feminine norm of dependency? Past research has been unable to disentangle these different types of ideal-discrepancies. 
Also complicating the interpretation of prior research on gender ideology and IPV involves the measurement of gender ideology. Both DeMaris et al. (2003) and Atkinson et al. (2005) used rather crude scales that were constructed post hoc from the available archival data. One study has used prospective data to evaluate the interactive effects of partners' gender ideologies on IPV (Cheung \& Choi, 2016), but even this scale was created for the purposes of the study and remains unvalidated. (It should be noted that the study in question, which sampled couples in Hong Kong, China, replicated the pattern of results found in DeMaris et al. [2003].)

As previously discussed, gender ideology is also frequently conflated with related concepts. A review of the literature found that studies measuring the link between egalitarianism or attitudes toward women's equal rights and IPV often produce mixed or null results (Moore \& Stuart, 2005). Thus, doubts regarding gender ideology's relevance to the problem of IPV could likely be resolved through better measurement. It will also be important to distinguish between masculinity/femininity ideology and global gender ideology (which includes both masculinity and femininity ideologies). Past research examining traditional masculinity ideology has found that it generally predicts sexual aggression and the perpetration of physical and psychological IPV (Levant \& Richmond, 2007; Moore \& Stuart, 2005). There appears to be no published research assessing femininity ideology and IPV.

It is also notable that the vast majority of the studies described in this section only assessed male-to-female IPV. If men are using IPV to gain or assert their dominance as prescribed by the patriarchy, then women subscribing to patriarchal beliefs should 
theoretically be less likely to perpetrate violence because they accept that their partner should hold more relational power. Violence also conflicts with the feminine role norms of deference and caretaking. However, women's gender ideology could be related to IPV perpetration in a different way: as a reaction to their partners' perceived failure to fulfill gender role norms. It is necessary to consider IPV perpetration by both men and women to fully understand the role of traditional gender ideology in violence.

The main study described in this manuscript examines a potential mechanism through which traditional gender ideology could predict IPV: its relationship to gendered partner-ideals. To the extent that a person holds traditional partner-gender ideology (i.e., a heterosexual man with traditional femininity ideology or a heterosexual woman with traditional masculinity ideology), they should also hold gendered partner-ideals. Thus, individuals strongly endorsing traditional gender ideology should also desire that their partners closely conform to gender role norms. As gendered partner-ideals increase in strength (i.e., become more important and desired), however, the larger the potential ideal-discrepancies. Low gender role conformity, then, could be a risk factor for IPV victimization among those who partners have strong gendered partner-ideals. Large partner-ideal discrepancies could motivate those partners to engage in regulation strategies including violence.

IPV, gender identity, and gender role conformity. Gender role conformity is also relevant to IPV apart from the fulfillment of gendered partner-ideals. Considering masculinity, recall that male role norms include power over women and aggression. While the former provides motivation for control behaviors in heterosexual relationships, 
the latter represents willingness to engage in physical violence. The norm of hypersexuality could also increase perpetration of sexual violence, even if harm is not intended (e.g., some men could misinterpret female partners' genuine reluctance or resistance as merely following sexual scripts). In contrast, women are expected to be deferent, kind, and care for others, characteristics that are not conducive to physical, psychological, or sexual violence. Given the vastly different domains encompassed by masculine and feminine role norms, respectively, it is important to differentiate between them in a discussion of violence.

As with the research on gender role conformity in other domains, much historic and contemporary work on gender role conformity in relation to violence utilizes the trait perspective of gender. Leonard and Senchak (1996) conducted an early but comprehensive study of trait masculinity, trait femininity, and other risk factors for premarital violence as well as violence during the first year of marriage. At the beginning of marriage, husbands' trait masculinity is unrelated to any IPV-relevant factors. Newlywed wives' trait femininity is negatively correlated with relational power, trait anger, and physical aggression. Thus, despite its association with low relational power, this study suggested that femininity could be generally protective against IPV perpetration.

The picture becomes more complex when multiple factors are considered simultaneously. Controlling for sociodemographics and various other factors (i.e., verbal aggression, problem-solving skills, withdrawal, anger, and alcohol use), premarital husband-to-wife physical IPV (wife-to-husband IPV was not considered) is uniquely and 
positively predicted by wives' low trait masculinity (Leonard \& Senchak, 1996).

Husbands' trait gender is not a significant predictor. One year after marriage, however, husbands' IPV perpetration is negatively related to husbands' trait femininity and unrelated to wives' trait gender. Thus, it may be that trait femininity decreases physical IPV perpetration, perhaps because of its relation to emotional awareness and relational warmth. The decreased risk of victimization associated with trait masculinity is more difficult to explain. Perhaps instrumentality lends itself to better outcomes in situations of conflict through superior problem-solving skills (a bivariate correlation Leonard and Senchak [1996] found in women but not in men). Or, perhaps the experience of victimization decreases self-perceived instrumentality over time.

Despite the findings of Leonard Senchak's (1996) study, other studies utilizing a trait perspective of gender have produced mixed results (Moore \& Stuart, 2005). In research using the instrumentality-expressiveness interpretation of trait gender, studies have found that abusive men have less trait femininity than nonabusive men, while trait masculinity is inconsistently related to IPV perpetration (Moore \& Stuart, 2005). However, this inconsistency could be a result of measurement specificity. Jenkins and Aube (2002) examined trait masculinity and femininity, but categorized characteristics within each as positive (i.e., socially desirable) or negative (i.e., socially undesirable). For both men and women, negative masculinity (e.g., egotism and greed) predicts increased IPV perpetration, while positive masculinity (e.g., independence and decisiveness) and positive femininity (e.g., gentleness and helpfulness) are associated with less IPV perpetration. 
It is important to point out that conformity to stereotypically gendered characteristics is a better predictor of aggression than is gender identity (Jenkins \& Aube, 2002; Richardson \& Hammock, 2007). In other words, the relationship between trait masculinity and violence is not explained by the fact that men tend to be higher in trait masculinity than are women; even women's trait masculinity predicts increased aggression. Similarly, trait femininity is more strongly related to aggression than is gender (although the negative association between trait femininity and aggression is less consistent across studies and effects are frequently null; Richardson \& Hammock, 2007). As suggested by the results of Jenkins and Aube (2002), different components of traditional masculinity or femininity may have different impacts on aggression. In other words, gender role conformity does not necessarily increase or decrease risk for IPV perpetration across the board. With the creation of instruments that measure specific gender role norms individually, such as the CMNI and CFNI, it has become possible to identify which norms are most relevant to aggression and IPV.

Similar to the research showing that trait masculinity predicts IPV, Cohn and Zeichner (2006) found that higher scores on the CMNI predicted men's willingness to administer stronger and more frequent shocks to a stranger with whom they believed they were in competition. Women's conformity to traditional male role norms also appears to be related to aggression; Reidy, Sloan, and Zeichner (2009) had female participants complete the CMNI, CFNI, and a measure of general physical and verbal aggressiveness before engaging in a sham aggression paradigm against a female opponent. Women's conformity to masculine role norms predicted both general aggressiveness and observed 
aggression with moderate-to-large effects. While women's conformity to feminine role norms negatively predicted general aggressiveness, it was unrelated to observed aggression. This difference in predictive ability could represent an unwillingness to report one's own aggressiveness. Alternatively, perhaps femininity inhibits aggression only in certain circumstances. Unfortunately, neither Cohn and Zeichner (2006) nor Reidy et al. (2009) reported results for individual gender role dimensions.

Some newer research has disaggregated the components of the male role. Using a scale similar to the CMNI, Sears (2011) examined the relationship between men's conformity to specific masculine role norms and various types of aggression. Global masculine role conformity was significantly related to physical and verbal aggression. Additionally, role-conforming men reported engaging in significantly more psychological and physical abusive behaviors toward any individual (i.e., not limited to intimate partners) in the past year. The individual norms of dominance, aggressiveness, and traditional views toward sex and sexuality (but not suppression of emotional vulnerability, avoiding dependency on others, or self-destructive achievement) were all related to both physical and verbal aggression. These same three role norms also predicted psychological and physical abuse behaviors, with the exception of dominance relating to physical abuse and with the addition of self-destructive achievement predicting infliction of injury to others. All significant correlations were positive, suggesting that conformity to male role norms - especially dominance, aggressiveness, and traditional views toward sex and sexuality — may contribute to aggressive patterns of behavior. While this study has the benefit of examining role norms separately, it did not examine unique 
contributions of role norms (i.e., the importance of individual role norms controlling for the effects of others). Nor is it clear that these results would generalize to abusive patterns of behavior within romantic relationships.

At least one study has examined the unique predictive ability of specific male role norms. Sampling around 100 men participating in batterer intervention programs (i.e., men who had perpetrated IPV) across three cities, Tager, Good, and Brammer (2010) collected data on self-reported abusive behaviors, difficulties in emotion regulation, and CMNI scores. Abusive behavior was significantly correlated with emotion dysregulation and global masculine role conformity as well as to the specific male role norms of emotional control, dominance, self-reliance, and power over women. When all factors were entered into a multiple regression equation predicting abusive behavior, emotion dysregulation (which was itself related to emotional control and self-reliance) and dominance together explained $25 \%$ of the variance. Thus, while traditional masculinity relates to aggression and IPV, certain norms are likely more harmful than others.

It has been established that conformity to masculine role norms predicts both general aggression (in men and women) and IPV perpetration (at least in men). While it is unknown whether conformity to feminine role norms predicts abusive behavior, feminine role conformity does predict attitudes toward abuse perpetration and the ability to recognize warning signs of abuse (Kearney, 2015). Specifically, the feminine norms of domestic, relational, involvement with children, and sexual fidelity each correlate with women's intolerance for men's abuse perpetration, while relational and involvement with children predict women's intolerance for women's abuse perpetration. Women high in 
relational, involvement with children, and sweet and nice are also better able to identity warning signs of IPV. Most of these norms prescribe pleasant, caring relational behavior, so women conforming to these norms could be more likely to expect reciprocal behavior from a romantic partner. Lastly, women's sexual fidelity predicts less experience with sexual coercion, while sweet and nice predicts less psychological aggression victimization. Thus, while some female role norms seem protective against IPV victimization, others could potentially reduce the risk of IPV perpetration through attitudes less tolerant of violence.

Men's conformity to masculine role norms paints a contrasting picture (Kearney, 2015). The norms of violence, power over women, and playboy each predict acceptance of men's abuse perpetration, with the former two norms also relating to acceptance of women's abuse perpetration. The norm of violence also predicts less ability to recognize the warning signs of abuse, likely because of increased acceptance of the behavior. Lastly, men high in self-reliance are more likely to experience psychological aggression, although there is no clear reason for this particular association.

While Kearney's (2015) study was comprehensive and identified specific gender role norms that could be beneficial or harmful, it also had several serious limitations that complicate interpretation. First, it was unclear whether the abuse victimization scales were only administered to participants in relationships - which included about half the sample — or to everyone. Administration to the entire sample would bias estimates of abuse downward (people cannot experience IPV if they have no intimate partner) and decrease statistical power to detect correlations with gender role norms. Second, the 
hierarchical regression analyses used to determine the ability of each gender role norm to predict attitudes toward violence (and the ability to recognize the warning signs of abuse) included a trait measure of gender that overlapped conceptually with the measures of gender role conformity. Thus, the findings discussed here were limited to bivariate correlations rather than the estimates of unique effects also reported by Kearney (2015). Third and finally, the sample was comprised entirely of undergraduate students. While this limitation is not uncommon, especially in research taking a more nuanced approach to gender role conformity, it might limit the generalizability of results.

Taken together, the research reviewed in this section suggests that conformity to masculine norms is positively related to IPV perpetration, while conformity to feminine norms - particularly those focusing on positive interpersonal behavior-generally negatively predicts IPV perpetration. More research is needed to determine which norms are most strongly related to IPV perpetration, as past research has been inconsistent.

Similarly, little is known about the link between conformity to gender role norms and IPV victimization. While research using the CMNI and CFNI suggest that conformity to masculine role norms is a risk factor for victimization while conformity to feminine role norms is protective (Kearney, 2015), trait measures of gender suggest that masculinity is protective while femininity is unrelated (Leonard and Senchak, 1996). This pattern of results is consistent with the idea that victimization is related to fulfillment of gendered partner-ideals. Trait measures of gender include almost exclusively socially desirable traits, so conformity should be positively received by most romantic partners and thus protective against or unrelated to victimization. In contrast, 
newer measures of gender role conformity such as the CMNI and CFNI also include neutral and potentially negative traits. Thus, the general desirability of a particular norm could be driving its association with victimization. For example, the feminine role norms of sexual fidelity and sweet and nice, which have been found to be protective against certain experiences with IPV (Kearney, 2015), are widely socially desirable. Other role norms, such as playboy, are more likely to displease a romantic partner. Under certain conditions, this displeasure could manifest as violence. Indeed, low relationship satisfaction has long been theorized as a risk factor for IPV.

IPV and relationship satisfaction. The correlation between relationship satisfaction and physical IPV is well-supported. Estimates in individual studies range widely, and a few studies have found support for a link between relational conflict— — but not relationship satisfaction — and IPV (Capaldi, Knoble, Shortt, \& Kim, 2012). This has led some researchers to conclude that the relationship between relationship satisfaction and IPV is likely due to increased conflict among distressed couples. However, a relatively recent meta-analysis of studies in heterosexual couples found that relationship satisfaction and its functional opposite, marital discord, both have a generally small-tomoderate correlation with IPV (around $r=-.27$; Stith, Green, Smith, \& Ward, 2008).

Several studies have found that gender is an important moderator in the relationship between IPV and relationship satisfaction. In the aforementioned metaanalysis, the relationship was stronger among male perpetrators and female victims (Stith et al., 2008). Newer research has confirmed that female victims are less satisfied in their relationships than are male victims (Ackerman \& Field, 2011; Kaura \& Lohman, 2007). 
This difference also exists in same-sex couples (Ackerman \& Field, 2011), suggesting that it is a matter of women's generally stronger reaction to victimization rather than gender differences in the type or severity of IPV perpetration. Collectively, these findings could mean that IPV serves different functions or has different meanings for women and men. However, without knowing the direction of causality, it is difficult to interpret the meaning of these gender differences.

While the vast majority of the research on relationship satisfaction and IPV has been cross-sectional rather than longitudinal, researchers have varied in their conceptualizations of directionality. It is possible that low relationship satisfaction precipitates violence (e.g., violence is used in retaliation or in an attempt to control the relationship to make it more satisfying), but it is also likely that the experience of violence decreases relationship satisfaction. Effects could also be bidirectional or different for different couples. There is also some evidence that relationship satisfaction may affect reporting rather than behavior; one study found that relationship satisfaction predicted over- or under-reporting psychological victimization compared to partner reports of perpetration (Marshall, Panuzio, Makin-Byrd, Taft, \& Holtzworth-Munroe, 2011). In other words, people who are in happier relationships might simply perceive less victimization or have more charitable perceptions of their partners' negative behaviors. The same study found some evidence for these effects in regards to physical IPV, but effects were inconsistent across samples. Perhaps it is more difficult to deny the experience of physical violence than psychological violence. 
A handful of studies have attempted to determine the causal direction of the association between IPV and relationship satisfaction by using longitudinal data. Most have found that aggression (i.e., physical IPV) is a precursor to decreased relationship satisfaction rather than the reverse. In other words, IPV may have a stronger impact on relationship satisfaction than relationship satisfaction has on IPV. Lawrence and Bradbury (2007) conducted the most comprehensive and sophisticated study on this topic, measuring physical IPV and relationship satisfaction in couples once a year for the first four years of marriage. Using growth-curve modeling, they found that husbands' IPV perpetration was associated bidirectionally with their own relationship satisfaction, although change in IPV was a stronger predictor of change in relationship satisfaction than the reverse. Associations in wives were weaker, with only initial IPV perpetration predicting initial relationship satisfaction and change in relationship satisfaction over time. However, victimization was unrelated to relationship satisfaction across time. This particular finding stands in contrast to the idea that victimization decreases relationship satisfaction, which was been suggested by other longitudinal research (e.g., Hammett, 2015; Panuzio \& DiLillo, 2010). Hammett (2015) measured IPV victimizationincluding physical aggression and intimidation — in married couples and found that both husbands' and wives' victimization during the first year of marriage negatively predicted wives' relationship satisfaction during the third year of marriage. Husbands' relationship satisfaction was unrelated to either their own or their wives' victimization. While the reasons for these differences were unclear, the author suggested that positive relationships between IPV perpetration and relationship satisfaction could be explained 
by increases in perpetrators' perceptions of their own relational power or control.

However, explanations for the longitudinal relationships between these constructs remain speculative.

Thus, while it appears more likely that physical IPV influences relationship satisfaction than the reverse, the variety of findings just described show that the causal relationship between relationship satisfaction and IPV is complex. It cannot be assumed that one predictably causes the other, as is implied by certain theoretical models. Firm conclusions are further precluded by studies finding a positive relationship between relationship satisfaction and IPV (Baker \& Stith, 2008; Testa, Hoffman, \& Leonard, 2011), meaning that supposedly happier couples are engaging in more violence.

Additionally, most research on this topic is limited to the perpetration of physical IPV. Perhaps low relationship satisfaction is a stronger motivator for non-physical IPV, such as sexual coercion or controlling behaviors. Clearly, further study is needed to clarify these complex and often conflicting findings.

IPV and partner characteristics. This section has already explored many facets of IPV: its prevalence, theorized causes, and relevance to relationship satisfaction. One topic that has been explored infrequently is the relationship between IPV and partner characteristics. There are a couple of different reasons why few researchers take this approach to studying IPV. First, it can be easily misinterpreted as victim blaming. It is assumed that identifying risk factors for victimization is equivalent to blaming IPV on those factors, partially or wholly absolving the perpetrator of responsibility. Second, identifying risk factors for victimization does not immediately suggest avenues for 
intervention in the same way that identifying risk factors for perpetration does. If it were found, for example, that a personality factor such as introversion or low self-esteem predicted victimization, it would be difficult to practically translate that knowledge into action or policy. Primary prevention based on individual risk factors for victimization would have to be broadly applied, and in many cases it would have questionable feasibility (e.g., attempting to intentionally change stable personality traits). There are also serious ethical concerns in attempting to change victims' characteristics or behaviors in the hopes of slightly decreasing the risk of future victimization. Such an approach would echo the controlling and disempowering dynamic of many abusive relationships.

Taken to the extreme, the good intentions behind these reasons for deprioritizing risk factors for victimization can result in a failure to acknowledge the reality that IPV happens in a relational context. Being able to identify individuals who are at increased risk of victimization based on their characteristics or on the combination of both partners' characteristics could improve current screening processes, prevention efforts, and approaches to intervention. Despite the discomfort that many researchers appear to feel with the issue, recognizing risk factors for victimization is not the same as assigning responsibility for the abuse.

Despite the unpopularity of focusing on victim characteristics, a number of risk and protective factors have been identified. In their review of the literature on risk factors for IPV, Capaldi et al. (2012) concluded that being relatively older and having social/emotional support are both protective against victimization. Conversely, witnessing IPV and being physically or sexually abused in childhood, using alcohol or 
drugs, being sexually jealous or possessive, and having attitudes supportive of violence are risk factors for victimization in adulthood (Capaldi et al., 2012; Renner \& Whitney, 2012). Adolescents with friends who perpetrate or experience IPV are themselves at increased risk for both (Capaldi et al., 2012). The logical connection between each risk factor and IPV is clearer for some than for others. Additionally, several "risk factors" may in fact represent effects of previous abuse rather than precursors, such as depressive symptoms or attitudes toward violence (Stith et al., 2004).

Several researchers have concluded that perpetrator characteristics are stronger predictors of violence within a relationship than are victim characteristics (e.g., Hotaling \& Sugarman, 1986; Stith et al., 2004), further decreasing interest in risk factors for victimization. If this conclusion is true, then prevention and treatment efforts focused on perpetration should be generally more efficient than those focused on victimization. However, one needs to consider the kinds of risk factors being measured in studies of victimization. Frequently, studies focus on individual characteristics drawn from family conflict theories (e.g., history of violence, substance use; e.g., Capaldi et al., 2012; Renner \& Whitney, 2012; c.f., Stith et al., 2004). Personal attributes other than psychopathology or family history, such as gender role conformity, are rarely measured. Additionally, most studies fail to consider relational context, or the interaction of both partners' attributes. Both of these factors could contribute to the small effect sizes of victimization risk factors. To illustrate, women's education and income each predict decreased victimization across studies with only small effect sizes $(d=-.10$ and -.09 ; Stith et al., 2004). However, results could be obscured by the failure to measure partners' 
corresponding partner-ideals. Recall that a woman's education and income can increase her risk of victimization if they conflict with the gender role attitudes - and thus presumably expectations - of her male partner (Anderson, 1997; Atkinson et al., 2005). Thus, whether a particular attribute increases, decreases, or has no relationship to the risk of victimization likely depends on the expectations and attitudes of the partner.

Patterns of change in IPV across relationships also suggest that IPV is influenced by interactive effects between partners rather than by perpetrator characteristics alone. Rather than being consistently intimately violent or nonviolent in all relationships across the lifespan, people appear to behave differently with different partners. Using longitudinal data that followed men from ages seventeen to 33, Shortt and colleagues (2012) found that while physical and psychological aggression are generally stable across time when men stay with the same partner, there is weak and frequently nonsignificant stability in aggressiveness across partners. While the sample of women was too small to allow for firm conclusions, their results show the same general pattern.

As discussed throughout this document, IPV is one potential strategy to exert control over a romantic partner, especially one who fails to fulfill partner-ideals. Past research has varied in the extent to which it has supported control as a self-identified motive for the perpetration of violence. Follingstad, Wright, Lloyd, and Sebastian (1991) found that $8 \%$ of male and $22 \%$ of female perpetrators identified control over the other person as a primary motive for their violence. Swan and Snow (2003) found that, among women who had aggressed against their partners in the past six months, $38 \%$ reported having used violence to control their partners - with the frequency varying widely 
depending on the directionality of coercion and physical violence in the relationship. Of these women, over half stated that the violence was effective at achieving control at least sometimes. While control is clearly far from the only self-reported motivation for violence, it has theoretical importance. Additionally, despite what perpetrators may perceive as their own primary motivations, research has supported need for control as a strong predictor of violence (Follingstad, Bradley, Helff, \& Laughlin, 2002).

Extending the idea that IPV is a strategy of control over partner's characteristics, one study has examined how ideal discrepancies predict IPV. Jaspaert and Vervaeke (2014) gathered data from both partners in 100 Dutch couples on IPV, relationship ideal discrepancies, relationship satisfaction, and communication. Participants who were perpetrators of psychological or physical violence or victims of psychological violence had larger relationship ideal discrepancies than those reporting no violence. Mediation analyses showed that ideal discrepancies did not have direct effects on psychological or physical perpetration or victimization. However, ideal discrepancies indirectly predicted physical violence perpetration and psychological violence victimization through conflict resolution skills. In contrast to the present study, Jaspaert and Vervaeke (2014) measured ideal discrepancies at the level of the relationship (which is influenced both partners) rather than at the partner level. Because the direct target of IPV is the partner rather than the relationship, it seems likely that partner-ideal discrepancies would be a stronger predictor than relationship ideal-discrepancies.

Gendered partner-ideal discrepancies represent a special type of potential risk factor for victimization. Unlike many of the previously identified risk factors for 
victimization, such as youth and having been abused as a child, many gendered characteristics are personally controllable behaviors. (That is not to say that they are all completely controllable; personality and genetics also surely have an influence.) In a previous section, we saw how much importance people place on a mate's gender role conformity. Combining this importance with the apparent controllability of gendered traits, gendered characteristics should be a likely target for control-motivated IPV. An actor who is displeased with the gendered characteristics of their partner (whether the partner conforms too much or too little) could use violence as punishment for the ideal discrepancy or more strategically to intimidate the partner into aligning their future behaviors with the actor's ideals. Which the latter is equivalent to coercive control in Johnson's typology of IPV $(1995 ; 2006)$, the former would most likely be situational couple violence. IPV in this context would represent a kind of backlash for gender role nonconformity. Importantly, the perpetrator would not necessarily experience their motivation as one for control. Instead, it could be perceived as anger, retaliation for a perceived slight (e.g., a woman's failure to be deferent could be perceived by her partner as emasculation), or frustration. Perpetrators report these motivations frequently (e.g., Bair-Merritt et al., 2010; Follingstad et al., 1991; Swan \& Snow, 2003). Many researchers have taken these reports at face value and concluded that violence is primarily a result of poor emotional self-control. However, identifying common causes of these emotions - including partner-ideal discrepancies—-would be incredibly useful for prevention and treatment. 
While violent reactions to gendered ideal discrepancies have not been researched in the context of romantic relationships, a handful of studies have examined the topic in the context of relationships with strangers or through hypothetical situations. Vandello and Cohen's (2008) work demonstrates that in cultures where a man's reputation is wrapped up in his wife's behavior, especially in her sexual purity, IPV is accepted as a legitimate way to restore honor lost from her transgressions. However, role violations do not have to be as extreme as infidelity to increase the risk of victimization. Reidy, Shirk, Sloan, and Zeichner (2009) had participants listen to an interview with a female confederate that covered each domain of the CFNI (e.g., "How important are romantic relationships?"). The confederate responded to all of the questions in a gender conforming or nonconforming way. Afterward, participants engaged in a sham aggression paradigm. While men who themselves conformed to traditional masculine role norms were more aggressive in both conditions, they were particularly aggressive toward nonconforming women. In contrast, nonconforming men displayed equal amounts of aggression to conforming and nonconforming women.

Berke, Sloan, Parrott, and Ziechner (2012) conducted a similar study in which men viewed a videotape of a feminine- or masculine-appearing female confederate providing demographic information about herself. Similar to the results of Reidy et al. (2009), gender-conforming men were generally more aggressive toward the confederate. However, unlike in Reidy et al. (2009), this effect was driven entirely by gender nonconforming men's decreased aggression toward the masculine confederate relative to the feminine confederate; gender conforming men were equally aggressive in both 
conditions. The authors interpreted this unexpected finding as possibly representing a prosocial reaction to being made aware of their own nonconformity. In other words, perhaps gender-nonconforming men were kinder to someone who they perceived as being similarly nonconforming. While Reidy et al. (2009) and Berke et al. (2009) found different patterns of results, both studies support the idea that actor gender role conformity interacts with the gender role conformity of the target to influence aggression. For obvious reasons, these experiments have not been conducted with actual romantic partners. Additionally, these studies have been limited to male perpetration. Little attention is paid to reasons for women's non-defensive violence (except in the family conflict literature, which generally assumes that men and women are influenced by the same factors).

To summarize, there is some existing research on risk factors for IPV victimization within real romantic relationships, the association between relationship ideal discrepancies and IPV, and the influence of target gender role conformity on men's willingness to aggress toward a stranger. However, these areas of exploration are disconnected. There is no work examining how gendered partner-ideals and partnerperceptions interact in real romantic relationships to predict the risk of violence.

Measurement of IPV. One major area of contention in the research on IPV involves the measurement of violence. The most popular instruments are the Conflict Tactics Scale (CTS; Straus, 1979), Revised Conflict Tactics Scale (CTS2; Straus, Hamby, Boney-McCoy, \& Sugarman, 1996), and various short forms adapted from either of these measures. On these instruments, respondents typically report the frequency with which 
they and/or their romantic partner engaged in specific conflict-relevant acts within a particular time frame, usually the preceding six months or one year. The original CTS contained the subscales of negotiation (a non-abusive conflict strategy, e.g., "suggested a compromise to an argument"), physical violence (e.g., "threw something at my partner that could hurt"), and psychological aggression (e.g., "insulted or swore at my partner"; Straus, 1979).

Data from the CTS and similar adapted measures have been handled in a few different ways. While some researchers treat IPV as dichotomous (typically treating a single reported violent act as the cutoff for abuse), others treat IPV as a continuous variable and account for both the number of unique abusive acts and the frequency. Alternatively, they may categorize abuse as mild, moderate, and severe.

The original CTS was heavily criticized for containing ambiguous items. For example, answering positively to "threw something at him/her" could mean that anything from a stuffed animal to a brick was thrown. Similarly, the original version failed to assess outcomes of violence. Researchers were skeptical of results showing equivalent rates of violence perpetrated by men and women, particularly given the evidence for different motivations and consequences based on gender (e.g., Follingstad et al., 2001; Nazroo, 1995). Partially in response to these critiques, the CTS2 added subscales for sexual coercion (e.g., "used force to make my partner have sex") and physical injury (e.g., "partner felt pain the next day"; Straus et al., 1996). Additionally, ambiguous items were made more specific (e.g., "threw something at a partner that could hurt"). 
The CTS and updated versions offer several advantages. The first of these comes from its popularity; because the CTS is so widely-used, its measurement properties are well-known and there is enough research to establish normative rates of violence within several different populations. Secondly, the format of the CTS allows collection of data on perpetration and victimization from both partners in a couple. This is important because a lack of agreement between partner reports (“dyadic concordance") can suggest under- or over-reporting. Past research has found limited agreement between partners (Browning \& Dutton, 1986; Marshall et al., 2011; Perry \& Fromuth, 2005). For individual items on the CTS, correlations between partners' reports have been found to vary from $r=.03$ to .80 , with most only moderately correlated (Browning \& Dutton, 1986). One study found moderate to large agreement for psychological, physical, and combined violence but small agreement for sexual coercion and injury (O’Leary \& Williams, 2006). Interestingly, both men and women underreported their own perpetration of physical aggression, sexual coercion, and injury; however, women also underreported male-to-female physical aggression (O'Leary \& Williams, 2006). Thus, depending on whether respondents were asked about perpetration or victimization, past research likely underestimates rates of women's victimization relative to men's victimization.

The difference in perceptions — or at least reports — of violence may be even more pronounced among severely abusive couples. One study sampling men involved in a batterer intervention program found that wives viewed husbands as nearly twice as physically abusive as the men perceived themselves (Browning \& Dutton, 1986). 
Husbands viewed themselves as moderately abusive and only somewhat more abusive than their wives! In other words, wives viewed themselves as the unidirectional target of violence while husbands viewed the relationship as mutually violent. To the extent that such severely abusive men are included in studies with representative samples, rates of bidirectional violence likely are inflated relative to male-to-female IPV. Measures of IPV that focus solely on victimization or perpetration risk lack the ability to identify these differences in perception and may result in erroneously high estimates of bidirectional violence relative to unidirectional violence.

Another benefit of the CTS involves the response options, which are meant to increase objectivity by soliciting a discrete count of abusive acts perpetrated or experienced within a specified timeframe. This format stands in contrast to measures using, for example, scales ranging from "never" to "frequently." Such scales are subjective and complicate interpretation of results. Perhaps, for example, individuals who suffer intense IPV victimization have come to expect that type of behavior from their partner and no longer view the behavior as notable. Such individuals would be more likely to under-report their victimization relative to an outside observer. Alternatively, due to cognitive biases, individuals who rarely perpetrate or experience violence may overestimate its frequency. Especially for couples in which violence is extremely infrequent (e.g., one instance of violence in the past year, which would preclude the use of the "never" response category), these subjective scales may decrease the accuracy and interpretability of results. 
Despite the CTS' stated goal of being an objective instrument, it is criticized for failing to consider context. For example, it is unclear whether a particular act listed on the CTS is used as a form of intentional abuse, in immediate retaliation or self-defense, or in response to a long pattern of victimization. Thus, it is possible that some victims would be erroneously categorized as perpetrators based on their decontextualized, selfreported behavior. Recall that Johnson $(1995,2011)$ proposed that there are different patterns of IPV. These patterns can be identified based on the severity of physical violence and controlling behaviors (Graham-Kevan \& Archer, 2003). Thus, one way to differentiate between different patterns of abuse is to simultaneously measure violence and controlling behavior. Together, this measures offer a much more complete picture of the dynamics of a given relationship.

In summary, the CTS is a very useful, if still imperfect, measure of IPV. The present study addresses some of the weaknesses of the CTS, such as the failure to acknowledge that abusive behaviors can occur in different contexts, by using an additional measure of emotional control behaviors.

\section{Gaps in the Literature}

The present study addresses many of the weaknesses with and gaps in prior research that I have identified throughout the literature review. One major area of weakness involves the measurement of gender-relevant constructs. Traditional gender ideology historically has been, and frequently still is, conflated with related but distinct constructs such as egalitarianism and sexism. Not only does the present study assess gender ideology using appropriate measures, but it also differentiates between 
masculinity and femininity ideology. While masculinity ideology has been explored in relation to physical and psychological health as well as certain relational attitudes and behaviors, very little is known about correlates of femininity ideology beyond the realms of psychopathology and disordered eating. The present study aimed to explore the importance of femininity ideology for romantic relationships.

The present study also extends previous research on gender role conformity. Readers will recall that early work using the trait perspective of gender treated masculinity as equivalent to instrumentality and femininity as equivalent to connectedness. More recent work found that gender role conformity is multidimensional. Thus, measures using the trait perspective only capture some of these dimensions. Because each role norm may be associated with unique outcomes, theorists have called for a disaggregation of gender role norms (Mahalik et al., 2005). However, only a handful of studies have analyzed individual role norms as unique predictors or outcomes. The present study is one of the first to analyze the association between specific masculine role norms and IPV as well as the first to attempt to examine the predictive ability of specific feminine role norms. Results could help resolve conflicting findings over whether femininity is protective against or unrelated to violence perpetration.

Readers will also recall that while past research on partner-ideals has frequently included the traditionally gendered components of attractiveness and resources, it has largely ignored other gendered domains. Currently, little is known about the extent to which people value their romantic partners' gender role conformity in a general sense, let alone in regards to specific domains. The present study is one of the first to treat 
gendered partner-ideals as multidimensional. It identifies which gendered partner-ideals are most strongly endorsed and most commonly fulfilled versus unfulfilled. Relatedly, the present study could potentially provide support for the presence of backlash against gender role nonconformity within a romantic relational context. Past research on backlash has been limited to hypothetical, casual, and work-based relationships. The pressure to conform to gender role norms in romantic relationships and the relational consequences of nonconformity are currently unknown.

Because it measures partner-ideals and partner-perceptions directly, the present study may also offer support for a parsimonious explanation of past research on gender ideology and IPV. Specifically, many have argued that traditional gender ideology predicts men's IPV perpetration in situations where men's power or status is threatened by their failure to have or earn more resources than their female partners. However, none of these studies actually measured the proposed causal mechanisms (e.g., feeling of threat to status, perceived failure to fulfill one's own gender roles). The present study proposes that rather than responding to a decrease in perceived power, these men could be using IPV to essentially punish and discourage their partners' violation of the feminine role norm of dependency/deference. The idea that IPV could be in reaction to perceived gender partner-ideal discrepancies also has the advantage of applying to IPV perpetrated by both men and women. In contrast, past research utilizing this framework has failed to consider women's IPV perpetration. Those theoretical perspectives that do examine female-perpetrated violence, such as the family violence perspective, usually ignore the 
potential relevance of gender-related constructs including gender ideology and gender role conformity.

At the same time, the present study is not limited to physical IPV; injury and emotional abuse are also considered. The majority of studies on IPV continue to focus on physical aggression to the exclusion of other forms. Additionally, while a handful of studies have considered emotionally controlling behaviors in the context of abusive relationships, they are rarely studied in otherwise non-violent relationships. Thus, there is exceedingly little information on the prevalence or severity of emotionally controlling behaviors in the general population.

A broad weakness of much of the literature reviewed in Chapter 1, especially in those studies dealing with gender ideology and gender role conformity, is its overreliance on undergraduate student samples. In many cases, it is unknown whether results generalize to the general adult population and especially to partners in long-term romantic relationships. Perhaps, for example, typical undergraduate students care less than older adults about the gender role conformity of potential partners because students are still in a period of role transition and have not settled into firm behavioral patterns. Maybe older adults, especially those who have had to navigate major turning points such as the birth of children or competing needs for advancement in each partner's career, feel differently about the importance of fulfilling traditionally gendered family roles. Past research has indeed suggested that gender ideology changes not over the lifespan, but also with life experience (Fan \& Marini, 2000). By sampling from the general adult population, the present research will be able to establish whether certain relationships 
found in previous research (e.g., the positive correlation between traditional gender ideology and traditional partner-ideals) generalize beyond undergraduate populations. 


\section{Chapter 2. Pilot Study to Develop Measures of Gendered Partner-ideals and}

\section{Partner-perceptions}

As discussed in Chapter 1, partner-ideal discrepancies involve the mismatch between partner-ideals and partner-perceptions. There currently exist no measures of partner-ideals or partner-perceptions relating specifically to gender roles. While the Ideal Standards Model contains two broad ideal domains relevant to gender (i.e., attractiveness-vitality and status-resources), it does not examine other components of male and female roles. Thus, measures of gendered partner-ideals and -perceptions were created for this study based on existing instruments assessing masculine and feminine ideologies, respectively (i.e., the MRNI-SF and the FIS). This chapter describes the process for creating and improving these measures.

\section{Measure Development}

Participants were asked to report on the gendered behaviors and characteristics of their partners. Thus, it might seem intuitive to adapt a measure of gender role conformity rather than gender ideology for this study. However, the CMNI and CFNI, the best available measures of gender role conformity, were not readily adaptable from a selfreport to a partner-report format. Many of the items focus on emotions and attitudes or hypothetical situations, which would be more difficult for a partner to accurately infer than actual (vs. hypothetical) and outwardly detectable behaviors. For example, the selfreport CFNI item "I would be ashamed if someone thought I was mean" could be adapted to the partner-report phrasing of "My partner would be ashamed if someone thought she was mean." However, responding to that item would be cognitively demanding because 
it requires the partner to infer another person's state of mind in a hypothetical situation. It would be preferable to measure the same construct - in this case "sweet and nice"with a behavioral item such as "My partner is nice to others." Additionally, current observable characteristics should be more directly related to relationship behaviors and experiences than hypothetical or internal attributes.

The MRNI-SF and FIS have the advantage of primarily focusing on behaviors. However, some items cannot be logically reworded to describe a romantic partner. An example is the MRNI-SF item "All homosexual bars should be closed down" from the negativity toward sexual minorities subscale. One option would be to reframe the item as "My partner believes that all homosexual bars should be closed down." However, this phrasing shifts the focus of the item away from the partner's actual gender role conformity to the partner's perceived attitudes regarding that role norm. In other words, believing that all homosexual bars should be closed down is not necessarily equivalent to behaving in a homophobic or even an overtly heterosexual way. To maintain the focus on conformity to the role norm rather than a partner's desire to achieve the role norm, most original MRNI-SF and FIS items based on emotions or attitudes were replaced with behavioral or physical attributes in the measures created for this study.

Some other items could not be logically adapted because they are too restrictive to accurately represent the construct of interest if reworded directly. For example, "An appropriate female occupation is nursing" is a good measure of the femininity ideology domain of caretaking, but obviously not all female partners who fulfill the caretaker role 
will be nurses. Thus, overly-specific gender ideology questions were expanded to include exemplars.

Another problem specific to the FIS involves the role norm of emotionality. As discussed in Chapter 1, these items are problematic because it is unclear whether they are assessing descriptive or injunctive norms. Someone might believe, for example, that "a woman will have a hard time handling stress without getting emotional." However, that descriptive belief is different than thinking that women in general or one's partner in particular should ideally have a hard time handling stress. Thus, items from the emotionality subscale that seemed unlikely to be widely endorsed as partner-ideals were reworded to include examples of emotionality that would likely be less detrimental to personal or relational functioning. Whenever possible, reworded items in both scales were based on items in the same domain from an earlier version of the MRNI-SF (for men only) or from the CFNI/CMNI. Before piloting the measures, items were discussed with the author's research team and colleagues and further edited based on that feedback.

\section{Hypotheses}

Hypothesis One (H1). Given that the measures of gendered partner-ideals and partner-perceptions were derived from existing measures of gender ideology, it was hypothesized that the factor structure of the new measures would reproduce those of the measures from which they were derived. However, the obtained sample size was too small to run a confirmatory factor analysis. Thus, it was hypothesized that internal consistencies would support the theoretical factors as distinct yet internally cohesive. A 
Cronbach's alpha of .70 or higher is typically considered acceptable (Cortina, 1993). Specifically:

H1a. Cronbach's alpha would be acceptable for the aggregate scales measuring feminine partner-ideals and partner-perceptions.

H1b. Cronbach's alpha would be acceptable for each of the subscales contained within the feminine partner-ideals and partner-perceptions measures (i.e., stereotypic images and activities, dependency/deference, purity, caretaking, and emotionality).

H1c. Cronbach's alpha would be acceptable for the aggregate scales measuring masculine partner-ideals and partner-perceptions.

H1d. Cronbach's alpha would be acceptable for each of the subscales (contained within the masculine partner-ideals and partner-perceptions measures (i.e., avoidance of femininity, negativity toward sexual minorities, self-reliance through mechanical skills, toughness, dominance, importance of sex, and restrictive emotionality).

Hypothesis Two (H2). Gendered partner-ideal discrepancies would negatively correlate with relationship satisfaction.

H2a. Aggregate feminine partner-ideal discrepancies would negatively correlate with men's relationship satisfaction.

H2b. Aggregate masculine partner-ideal discrepancies would negatively correlate with women's relationship satisfaction.

\section{Method}

\section{Participants}


Individuals aged 18 or older who identified as male $(n=25)$ or female $(n=71)$ and who were in a relationship with someone identifying with the other gender participated in the pilot study. Three people were excluded from analyses because they indicated that they provided poor-quality data, including one person who admitted that she was not actually in a relationship and only completed the survey because she wanted extra credit. Four women were in polyamorous relationships but responded in regards to a male-identified partner; their data were retained for analyses.

Participants in the final sample ranged from eighteen to 59 years old $(M=26.8$, $S D=7.7)$. The majority $(70.8 \%)$ described themselves as straight or heterosexual, $14.6 \%$ as bisexual, and $8.3 \%$ as other (including two as asexual). The majority (68.8\%) were White, followed by Hispanic or Latino (8.3\%), bi- or multiracial (8.3\%), Asian $(5.2 \%)$, Pacific Islander (1.0\%), or a different race $(2.1 \%) ; 6.3 \%$ did not report their race. Frequencies of partner races were nearly identical; $69.8 \%$ reported that their partner was White, followed by Hispanic or Latino (8.3\%), bi- or multiracial (7.3\%), Asian $(3.1 \%)$, Black (1.0\%), Native American (1.0\%), or a different race (3.1\%), with $6.3 \%$ leaving the question unanswered. Most participants (68.8\%) were undergraduates, although $15.6 \%$ were graduate students, $7.3 \%$ were post-baccalaureate students, $2.1 \%$ were not students, and $6.3 \%$ did not specify their student status.

Twenty percent of participants were married, and the remaining $80 \%$ were in a romantic relationship. While $17.8 \%$ had been in their romantic relationship for six months or less, $71.8 \%$ had been in the relationship with their partner for at least one year. 
Just under half (49.0\%) lived with their romantic partner; the rest did not. Only $11.5 \%$ of participants reported having children.

\section{Measures}

Feminine partner-ideals and partner-perceptions. As described above, measures of feminine partner-ideals and partner-perceptions (see Appendix A) were created based on the FIS (Levant et al., 2007). Each measure asked respondents to rate 47 items, two of which were potential alternate adaptations of the same FIS item. For the partner-ideals measure, participants were instructed, "Please take a moment to imagine the perfect romantic partner. Rather than thinking about what most people would want in a partner, consider what you personally would prefer a romantic partner to be like. Think about your ideal romantic partner. My ideal partner would..." Items were rated on a scale from $1=$ Strongly disagree to $5=$ Strongly agree. For partner-perceptions, participants were told, "Now, please consider your current romantic partner. Please indicate how well the following characteristics describe your current partner. My partner..." Items were rated using the same scale as for partner-ideals.

Masculine partner-ideals and partner-perceptions. Measures of masculine partner-ideals and partner-perceptions (see Appendix B) were based on the MRNI-SF (Levant et al., 2013); thus, respondents rated 21 items. Items were rated on a scale from $1=$ Strongly disagree to $7=$ Strongly agree. Instructions for the masculine partner-ideals and partner-perceptions measures were the same as for the equivalent feminine measures.

Gendered partner-ideal discrepancies. There are many different possible approaches to quantifying discrepancy. One alluringly intuitive option is the use of 
difference scores. For the present study, difference scores would involve simply subtracting the partner-perceptions score on a given item from the equivalent partnerideals item; the result would represent the degree of mismatch. However, this approach suffers from many problems (e.g., Cafri, van den Berg, \& Brannick, 2010; Edwards, 2001; Griffin, Murray, Gonzalez, 1999). Most of the problems with difference scores stem from the loss of information created by combining two different values that have their own means and variances; this creates the opportunity for confounded effects. If a difference score between partner-ideals and -perceptions predicts an outcome, there is no way to know whether that statistical relationship is being driven by the partner-ideals scores, the partner-perceptions scores, or a combination of both. For example, knowing that participants' difference scores are small gives no information about the magnitude of their partner-ideals or partner-perceptions. Perhaps they do not hold gendered partnerideals, or perhaps they all perceive their partners as highly role-conforming. As noted in Fletcher et al. (2000), the use of within-subjects correlations has the benefit of producing a single score that is not confounded with positivity biases (i.e., positive illusions of a romantic partner). Setting aside interpretation of the magnitude of difference scores, there are also conceptual issues concerning the interpretation of the scores' signs. For example, is over-conforming and under-conforming to role norms relative to ideals (which would be reflected in negative or positive difference scores, respectively) equally detrimental to RS? This kind of question must be answered empirically, but the use of difference scores implies that discrepancies should the same outcomes regardless of their directionality. 
A related alternative to difference scores is to ask participants to quantify the extent to which their partners meet their ideals on a given dimension. Edwards (2001) thoroughly criticizes this approach. Not only does it suffer from the same interpretation problems as difference scores, but it also results in a loss of available informationincluding statistics on partner-ideals and -perceptions, which are themselves informative - by shifting the burden of the difference calculation onto the participant. Direct comparison items are in fact double-barreled because they ask respondents to consider two distinct concepts simultaneously. Most concerning is the empirical research showing that direct comparison items sometimes yield different results than calculated differences between items assessing ideals and perceptions. This finding suggests that respondents are not necessarily systematic in their internal comparison of ideal and perceived attributes.

Yet another option is to use a product term that models the interaction between ideals and perceptions. However, as shown by Edwards (2001), this approach does not actually model the effects of congruence when measures are continuous (as in the present study). Additionally, the interpretation does not accurately reflect the construct of interest because it effectively focuses on differences between people; a significant product term would mean that differences in the outcome rely on how strongly a person holds gendered partner-ideals and how much a partner is perceived as conforming to gender role norms relative to other participants in the sample. For example, a small partner-ideal discrepancy would be represented by holding strong feminine partner-ideals relative to other participants and perceiving a partner as being relatively more feminine 
than others participants their partners to be. Eastwick and Neff (2012) describe this as the "level" approach because it focuses on the relative levels of ideals and partner traits.

In contrast, the present study uses what Eastwick and Neff (2012) describe as the "pattern approach," which focuses on the general congruence of the constellations of ideals and perceptions. In this framework, the important factor is how closely individuals' ideals align with their partner-perceptions in regards to a given trait. In terms of the present study, the pattern approach predicts that the outcome (relationship satisfaction) will be a function of the general congruence between gendered partner-ideals and partnerperceptions. Practically speaking, in the pattern approach, having a partner grossly overconform to gender role norms relative to one's ideals (e.g., having a very homophobic partner despite desiring a partner who is not at all homophobic) would be considered as negative as under-conforming (e.g., being less homophobic than desired). In their comparison of the pattern and level approaches, Eastwick and Neff (2012) found that while this "pattern" approach to ideal-discrepancies predicts divorce, the "level" approach does not.

In the present study, gendered partner-ideal discrepancies were calculated following the procedures outlined in Fletcher, Simpson, and Thomas (2000), which follows a "pattern" approach. Specifically, within-subjects correlations were calculated between scores on the partner-ideals and partner-perceptions measures. Next, these scores were multiplied by negative one so that higher scores indicated a greater degree of discrepancy rather than convergence. 
Relationship satisfaction. Relationship satisfaction was assessed using scores on two different scales: the Revised Dyadic Adjustment Scale (RDAS; Busby, Crane, Christensen, \& Larson, 1995) and Relationship Assessment Scale (RAS; Hendrick, 1988). The RDAS consists of fourteen items assessing degree of agreement on important issues, frequency of regret and irritation, engagement in shared activities, and positive interactions. One item (i.e., "Do you ever regret that you married (or lived together)?") was slightly altered to make it applicable to individuals in non-cohabitating dating relationships (i.e., "Do you ever regret that you married, lived together, or got together?"). All items are scored on a six-point scale where 0 indicates low relationship quality and 5 indicates high relationship quality. The RDAS has three subscales: consensus, satisfaction, and cohesion. Past research has found that the overall scale has a Cronbach's alpha of .90 , with alphas for the subscales ranging from .80 to .85 (Busby et al., 1995). Cronbach's alpha in the present study was .85 for the overall scale, .68 for consensus, .84 for satisfaction, and .71 for cohesion.

While the RDAS has good evidence of construct and criterion validity (Busby et al., 1995), some of its items focus on behaviors (e.g., engaging in outside interests together) rather than subjective evaluations of the relationship. Because these outcomes are theorized to be more distal potential outcomes of partner-ideal discrepancies (which would bias correlations in Hypothesis Two downward), a purely subjective measure of relationship satisfaction, the RAS, was also used in this study. The RAS is a seven-item scale measuring general relationship satisfaction (e.g., "In general, how satisfied are you with your relationship?"). Respondents rate each item on a scale from $1=$ Low to $5=$ 
High, where higher numbers indicate greater relationship satisfaction. Two items assessing dissatisfaction are reverse-scored (e.g., How often do you wish you hadn't gotten into this relationship?"). Past research has found that the RAS has high internal consistency (Cronbach's alpha $=.86$ ) and has moderate-to-strong correlations with other measures of relationship quality (Hendrick, 1988). In the present study, Cronbach's alpha was .90.

Demographics and relationship information. Participants also answered questions about their current and prior relationships and provided demographic information (e.g., age, race, sexual orientation, education, student and employment status; Appendix B).

Data quality questions. At the end of the questionnaire, participants were asked whether they encountered any technical problems with the survey and whether, in their opinion, their data should be used in analyses (Appendix B). Participants were assured that their compensation would not be affected by their response.

Procedure. The pilot study was approved by the university Human Subjects Research Review Committee. Participants were recruited through Portland State University using flyers posted on campus and through direct invitations to students in their classes. Individuals were invited to participate if they were currently in a romantic relationship. To encourage participation, respondents could enter into a random drawing for one of two \$25 Amazon.com gift cards or earn extra credit in certain classes (an alternative extra credit assignment was also offered in these classes). Data were collected between July and October of 2016. 
After consenting to participate (Appendix C) and passing a brief eligibilityscreening questionnaire (Appendix D), respondents completed questions assessing their gendered partner-ideals, gendered partner-perceptions, and relationship satisfaction. The gendered partner-ideals and partner perceptions measures were presented in a counterbalanced order across participants. Additional measures regarding gender and relationship dynamics were administered but are not discussed in this document. At the end of the survey, participants provided demographic information and answered a few brief data quality questions (Appendix B).

\section{Results and Discussion}

\section{Hypothesis One}

Hypothesis One examined the internal consistency of each of the measures and their subscales. Means, standard deviations, Cronbach's alphas, and subscale intercorrelations are reported in Table 1 for the feminine partner-ideals and partnerperceptions measures and in Table 2 for the masculine partner-ideals and partnerperceptions measures.

H1a. The aggregate feminine partner-ideals measure had a Cronbach's alpha of .95, and the aggregate partner-perceptions measure had an alpha of .77. This suggests that both measures have acceptable internal consistency.

H1b. Cronbach's alphas for the subscales ranged widely (see Table 1). For the feminine partner-ideals subscales, alpha ranged from .66 for emotionality (the only subscale below the predetermined cutoff of .70 as "acceptable") to .89 for purity. 
With one exception, alphas for the feminine partner-perceptions subscales were lower than for the equivalent partner-ideals; alphas ranged from .32 for stereotypic images and activities to .77 for purity. Three of the five subscales (i.e., stereotypic images and activities, dependency/deference, and caretaking) had below-acceptable alphas.

To identify potentially problematic items, I examined interrcorrelations of the items within each subscale and identified those items with low or negative interrcorrelations. Problematic items were discussed with other researchers knowledgeable about gender roles. Some items were edited to map more closely onto the construct of interest. For example, the original FIS caretaking item "An appropriate female occupation is nursing" was adapted to "[My ideal partner would] hold a traditionally feminine job like nurse, homemaker, etc." for the partner-ideals measure and to "[My partner] holds a traditionally feminine job like nurse, homemaker, etc." for the partner-perceptions measure. This item had low intercorrelations with the other items in the subscale, particularly for partner-perceptions. There are many potential reasons why this item could have been problematic. First, the fact that there are a limited number of highly stereotypically-feminine jobs could have contributed to a lack of variance in the item (less than $10 \%$ of participants responded with agreement). Second, there are many forces other than personality that influence which job a person holds. Given that the sample largely consisted of college students, it seems likely that those partners who were working at all (rather than studying) probably took jobs more for their availability than their career-building potential. For these reasons, the item was broadened to "[My 
partner] takes care of others when they're sick or need help" and [My ideal partner would" take care of others when they're sick or need help," which retains the core ideas underlying the original item. Other problematic items, particularly those that were direct adaptations from the FIS, were augmented with additional adaptations.

H1c. Cronbach's alpha was .91 for the aggregate masculine partner-ideals measure and .79 for the aggregate partner-perceptions measure, demonstrating good internal consistency.

H1d. Cronbach's alphas for the masculine partner-ideals and-perceptions subscales ranged widely (see Table 2). For masculine partner-ideals, alpha ranged from .15 for negativity toward sexual minorities to .87 for both self-reliance through mechanical skills and importance of sex. In addition to negativity toward sexual minorities, the subscales of avoidance of femininity and toughness also had belowacceptable alphas.

For the masculine partner-perceptions subscales, alphas ranged from .50 for negativity toward sexual minorities to .83 for importance of sex. The subscales of avoidance of femininity and toughness also had below-acceptable alphas. However, for both the partner-ideals and-perceptions measures, it is possible that subscale alphas were biased downward because of the small number of items in each scale (i.e., three); Cronbach's alpha is sensitive to the number of items (Cortina, 1993). Additionally, with such small subscales, one poorly-fitting item can easily drag down the alpha. For example, one partner-ideal item assessing negativity toward sexual minorities, "[My ideal partner would] never compliment or flirt with another male," had negative correlations 
with the two other items in the subscale. Without the item, the subscale alpha would increase from a very low .15 to .50 . It was decided that the problem with this particular item might have been the inclusion of "compliment," which moves away from the construct's focus on sexuality. Thus, it was changed to "[My ideal partner would] never flirt with another man.”

The process for revising items was the same as that described above for the equivalent feminine measures. Because achieving sufficient internal consistency was such a concern in the small subscales, several problematic items were augmented with additional adaptations for the final versions.

\section{Hypothesis Two}

Hypothesis Two stated that gendered partner-ideal discrepancies would negatively predict relationship satisfaction. This hypothesis was tested using the piloted measures (i.e., without the changes or additional items discussed above). Descriptive statistics and correlations are provided in Table 3. The mean within-person correlation for the partnerideals and perceptions measures was .49 for male participants and .48 for female participants, suggesting that most people perceived their partners as generally matching many of their gendered ideals.

H2a. Men's feminine partner-ideal discrepancies negatively correlated with RDAS scores, $r(22)=-.67, p=.001$, and RAS scores, $r(22)=-.59, p=.004$. Feminine partner-ideal discrepancies were also negatively correlated with each subscale of the RDAS (see Table 3). 
H2b. Similarly, women's masculine partner-ideal discrepancies were negatively correlated with RDAS scores, $r(65)=-.55, p<.001$, RAS scores, $r(65)=-.50, p<.001$, and each subscale of the RDAS (see Table 3).

In summary, Hypothesis 2 provides support for the idea that gendered partnerideal discrepancies are related to relationship satisfaction. Specifically, smaller discrepancies between gendered partner-ideals and partner-perceptions predicts higher relationship satisfaction. These results provide preliminary supporting evidence for some of the research questions explored in the next chapter. 


\section{Chapter 3. Pilot Study to Examine Factor Structure of Measures of Gendered}

\section{Partner-ideals and Partner-perceptions}

The pilot study described in the previous chapter gave some insight into the measurement properties of the measures of feminine and masculine partner-ideals and perceptions created for the present study. It also allowed for the identification of some items with problematic internal consistency. However, the obtained sample was too small to explore the factor structures of the created measures.

This chapter describes a larger pilot study completed to examine the factor structures of the created measures and to determine the degree of internal consistency in each subscale. The primary goal was to see whether the underlying factor structure of the created measures contained the same dimensions as those of the respective gender ideology measures on which they were based. While the created measures have not been previously factor analyzed, confirmatory factor analysis is more appropriate than exploratory factor analyses in situations when there is a theory guiding the hypothesized structure of the variables (Tabachnick \& Fidell, 2001). Additionally, confirmatory factor analysis has the advantage of allowing for the assessment of overall measurement model fit.

This chapter also confirms a facet of predictive validity that was explored in the previous pilot study: whether partner-ideal discrepancies—-measured using the created partner-ideals and -perceptions measures-predict relationship satisfaction. Relationship satisfaction is assessed using a different measure than in the previous chapter for reasons that are explained below. 


\section{Hypotheses}

Hypothesis One (H1). Because the feminine partner-ideals and-perceptions measures were based on the Femininity Ideology Scale (Levant et al., 2007), it was expected that both created measures would contain equivalent factors to those on the FIS. Additionally, it was expected that each factor would demonstrate acceptable internal consistency. Specifically:

H1a. Fit indices for confirmatory factor analyses would support an oblique, five-factor model of feminine partner-ideals and -perceptions with the distinct factors of stereotypic images and activities, dependency/deference, purity, caretaking, and emotionality. Adapted items would load onto the specific factor to which they were intended (e.g., an item adapted from the purity subscale of the FIS would load onto the respective purity feminine-ideal and - perception factors).

H1b. Internal consistency coefficients would be acceptable $(\geq .70)$, using the guidelines put forth by Kline (2011), for each of the hypothesized factors contained within the feminine-ideals and -perceptions measures.

Hypothesis Two (H2). Because the masculine-ideals and -perceptions measures were based on the Male Role Norms Inventory - Short Form (Levant et al., 2013), it was expected that the created measures would contain the same theorized dimensions. It was also expected that each factor would demonstrate acceptable internal consistency. Specifically:

H2a. Fit indices for confirmatory factor analyses would support an oblique, sevenfactor model of masculine partner-ideals and -perceptions with the distinct factors 
of avoidance of femininity, negativity toward sexual minorities, self-reliance through mechanical skills, toughness, dominance, importance of sex, and restrictive emotionality. Adapted items would load onto the factor to which they were intended.

$\mathrm{H} 2 \mathrm{~b}$. Internal consistency coefficients would be acceptable $(\geq .70)$ for each of the hypothesized factors contained within the masculine partner-ideals and partnerperceptions measures.

Hypothesis Three (H3). Based on the Ideal Standards Model, larger partnerideal discrepancies should predict lower relationship satisfaction. Hypothesis Three tested whether gendered partner-ideal discrepancies, measured using the created measures of gendered partner-ideals and -perceptions, negatively correlate with relationship satisfaction. It was hypothesized that:

H3a. Aggregate masculine partner-ideal discrepancies would negatively correlate with women's relationship satisfaction.

H3b. Aggregate feminine partner-ideal discrepancies would negatively correlate with men's relationship satisfaction.

\section{Method}

\section{Participants}

Individuals were eligible to complete the pilot questionnaire if they were currently living in the United States, were aged 18 or older, identified as cisgender male or female, and were in a heterosexual romantic relationship (i.e., in a relationship, married, or in a domestic partnership) of at least six months with another adult. 
The final sample consisted of $n=266$ women and $n=377$ men. With 25 items administered on the masculine measures and 47 items administered on the feminine measures, these samples are within the ratios of 5:1 - 10:1 cases per free parameter tentatively proposed by Bentler and Chou for confirmatory factor analysis (1987). However, there are no definitive rules for sample size in CFA; the ideal sample size depends upon a number of factors including the number of indicators, size of the factor loadings, and multivariate normality of the data (Bentler \& Chou, 1987; Tabachnick \& Fidell, 2007). MacCallum, Browne, and Sugawara (1996) stated that with 100 degrees of freedom in a model, statistical power for RMSEA values approach .87 with a sample size of 200 and approach 1.00 with a sample of 400 . By these estimates, both CFAs should be adequately powered. Because men and women completed different measures, sample characteristics are reported separately by gender below. It should also be noted that not everyone who completed the partner-ideals scales finished the entire survey, so sample sizes for some descriptive statistics and inferential tests are smaller.

The final sample of female participants ranged from nineteen to 65 years old $(M=$ $34.3, S D=10.2)$. The majority $(90.6 \%)$ described themselves as straight or heterosexual, $7.1 \%$ as bisexual, and $2.3 \%$ did not report their sexual orientation. Participants were only required to be in a heterosexual relationship and thus presumably attracted to individuals of the other sex; they were not required to have an exclusively heterosexual orientation. The majority (70.3\%) was White, followed by Black or African American (10.9\%), Asian (6.4\%), Latino or Hispanic (3.0\%), or Other (.8\%); while $6.4 \%$ reported two or more categories and $2.3 \%$ did not report their race. Frequencies of partner races were similar; 
$65.8 \%$ reported that their partner was White, followed by Black or African American (11.7\%), Latino or Hispanic (5.3\%), Asian (4.9\%), Native American (.4\%), and Other (.4\%); with $9.4 \%$ reporting two or more categories and $2.3 \%$ missing data. The highest level of obtained education varied widely, with $1.1 \%$ having some high school or less, 26.7\% having a high school diploma or GED, 21.4\% holding an Associate's or occupational degree, $35.7 \%$ holding a Bachelor's, 10.2\% holding a Master's, $1.9 \%$ holding a professional school degree, and $.8 \%$ with a doctoral degree. Reported annual personal income (assessed separately from the partner's income) ranged from $\$ 0$ $150,000(M=\$ 27,365.95, S D=25,580.97, \mathrm{Med}=\$ 20,000)$.

Among women, $60.9 \%$ were married or in a domestic partnership and the other $39.1 \%$ were in a romantic relationship. The length of these participants' relationships ranged from six months to 51.6 years $(M=9.4$ years, $S D=9.1, M e d=6.3)$. Those who were married or in a domestic partnership had been married or domestically partnered for an average of 10.4 years $(S D=9.5, \mathrm{Med}=7.5)$. Eighty-five percent currently lived with their romantic partner, while the other fifteen percent did not. While over half $(58.3 \%)$ had one or more child, just under half (49.6\%) lived with one or more children under 18. The majority of female participants $(85.0 \%)$ reported taking the survey alone, while $12.4 \%$ said their partner was nearby but couldn't see how they answered, $.4 \%$ said their partner was nearby and could see how they answered, and $2.3 \%$ exited the survey before the question was asked.

Male participants ranged from 19 to 80 years old $(M=35.2, S D=10.5)$. The majority $(91.5 \%)$ described themselves as straight or heterosexual, $2.1 \%$ as bisexual, .5\% 
as a different sexual orientation, and $5.8 \%$ did not report their sexual orientation. The majority (69.5\%) was White, followed by Asian (9.8\%), Black or African American (5.8\%), Latino or Hispanic (5.6\%), and Native American (1.1\%); while 2.4\% reported two or more categories and $5.8 \%$ did not report their race. Frequencies of partner race/ethnicity were similar; $67.4 \%$ reported that their partner was White, followed by Asian (9.8\%), Black or African American (6.1\%), Latino or Hispanic (5.8\%), Native American (.8\%), and Pacific Islander (.5\%); with 3.7\% reporting two or more categories and $5.8 \%$ missing data. The highest level of obtained education varied widely, with $.3 \%$ having some high school or less, $20.4 \%$ having a high school diploma or GED, $15.4 \%$ holding an Associate's or occupational degree, 37.1\% holding a Bachelor's, 16.7\% holding a Master's, 3.2\% holding a professional school degree, and $1.1 \%$ with a doctoral degree. Reported annual personal income ranged from $\$ 0-250,000(M=\$ 48,198, S D=$ $34,074, \mathrm{Med}=\$ 40,000)$.

Among male participants, $62.6 \%$ were married or in a domestic partnership and the other $37.4 \%$ were in a romantic relationship. Reported relationship lengths ranged from six months to 48.2 years $(M=8.6$ years, $S D=8.5, M e d=6.2)$. Those who were married or in a domestic partnership had been married or domestically partnered for an average of 9.4 years $(S D=9.1, \mathrm{Med}=6.8)$. Seventy-nine percent currently lived with their romantic partner, while the other $21 \%$ percent did not. The majority $(54.9 \%)$ had no children, and less than half (41.6\%) lived with one or more children under 18 . The majority of male participants $(80.4 \%)$ reported taking the survey alone, while $12.2 \%$ said their partner was nearby but couldn't see how they answered, 1.3\% said their partner was 
nearby and could see how they answered, and $6.1 \%$ exited the survey before the question was asked.

\section{Measures}

Feminine partner-ideals and partner-perceptions. As described in the previous chapter, measures of feminine partner-ideals and partner-perceptions (see Appendix A) were created based on the FIS (Levant et al., 2007). Each measure asked respondents to rate 47 items, two of which were potential alternate adaptations of the same FIS item. For the partner-ideals measure, participants were instructed, "Please take a moment to imagine the perfect romantic partner. Rather than thinking about what most people would want in a partner, consider what you personally would prefer a romantic partner to be like. Think about your ideal romantic partner. My ideal partner would..." Items were rated on a scale from $1=$ Strongly disagree to $5=$ Strongly agree. For partner-perceptions, participants were told, "Now, please consider your current romantic partner. Please indicate how well the following characteristics describe your current partner. My partner...” Items were rated using the same scale as for partner-ideals. Masculine partner-ideals and partner-perceptions. Measures of masculine partner-ideals and partner-perceptions (see Appendix A) were based on the MRNI-SF (Levant et al., 2013). Five items that were identified in Chapter 2 to be potentially problematic were augmented with an additional adapted item; thus, respondents rated 26 items on a scale from $1=$ Strongly disagree to $7=$ Strongly agree. Instructions for the masculine partner-ideals and partner-perceptions measures were the same as for the equivalent feminine measures. 
Gendered partner-ideal discrepancies. Gendered partner-ideal discrepancies were calculated as described in Chapter 2. Specifically, within-subjects correlations were calculated between scores on corresponding partner-ideals and partner-perceptions items. Next, these scores were multiplied by negative one so that higher scores indicated a greater degree of discrepancy rather than convergence.

Relationship satisfaction. Relationship satisfaction was assessed using the Perceived Relationship Quality Components Inventory (PRQC; Fletcher, Simpson, \& Thomas, 2000b). In this instrument, respondents rate how well eighteen items describe their relationship or partner (depending on the specific domain under consideration) on a scale from $1=$ Not at all to $7=$ Extremely. The PRQC covers six dimensions: relationship satisfaction (e.g., "How satisfied are you with your relationship?"), commitment (e.g., "How dedicated are you to your relationship?"), intimacy (e.g., "How connected are you to your partner?"), trust (e.g., "How much can you count on your partner?"), passion (e.g., "How sexually intense is your relationship?"), and love (e.g., "How much do you love your partner?"). Item ratings are summed to create an overall score for relationship satisfaction.

While many other instruments assessing relationship satisfaction or quality (terms that many researchers use interchangeably) include behavioral components (e.g., "How often do you work together on a project?"), the PRQC focuses only on cognition. This characteristic makes it highly appropriate for the present study. The present study focuses on the potential impacts of gendered partner-ideal discrepancies (which are subjective and cognitive), so measuring subjective, cognitive evaluations of romantic 
relationships represents a better match between the levels of the constructs. The exclusion of behavioral domains also helps avoid the confounding of process and outcome in relationship dynamics. For example, domains commonly included in other measures of relationship satisfaction/quality, such as shared activities and experiences of conflict, could be either cause or consequence of a less favorable view of the relationship.

Confirmatory factor analysis of the PRQC has shown that each conceptual domain represents a distinct factor, while all domains also load on a second-order latent relationship quality factor (Fletcher et al., 2000b). Thus, while individuals do have a general sense of the relationship quality, they are also able to evaluate their partners on separate domains. In past research, alpha reliabilities for each component of relationship quality have ranged from .78 to .96 (Fletcher et al., 2000a). Cronbach's alpha for the total scale in this pilot study was .96 for both women and men.

The PRQC has good criterion and predictive validity. In the area of partner-ideals, Fletcher et al. (2000b) found that smaller partner-ideal discrepancies are associated with higher relationship quality, a correlation that increases in strength over time in new relationships (participants were followed three months from the first month of a relationship). Smaller-ideal discrepancies are also associated with higher relationship quality in couples that have been together at least six months (Overall et al., 2006). The PRQC has also demonstrated criterion validity in regards to relational factors; scores are related to the perceived mutuality of relationship goals and progress toward attaining those goals (Avivi, Laurenceaue, \& Carver, 2009), partners' provision of positive support 
(Overall, Fletcher, \& Simpson, 2010), and healthy communication patterns (Givertz \& Safford, 2011).

Demographics and relationship information. Participants also answered questions about their current and prior relationships and provided demographic information (Appendix B).

Data quality questions. At the end of the questionnaire, participants were asked whether they encountered any technical problems with the survey. They were also asked about the setting in which they completed the survey (i.e., alone, with their romantic partner nearby but unable to see their responses, or with their partner nearby and able to see their responses). This question was included to give an idea of the social context in which participants were completing the questionnaire. It was anticipated that the presence of romantic partners during survey self-administration could potentially influence responses on the measures of IPV collected in the final survey (see Chapter 4). As a self-reported measure of data quality, participants were asked whether, in their opinion, their data should be used in analyses (Appendix B). The instructions assured participants that their compensation would not be affected by their response.

Procedure. The pilot study was approved by the university Human Subjects Research Review Committee. Participants were recruited through Amazon's Mechanical Turk in July of 2017 and paid $\$ 0.20$ for completing the survey. Participants who passed a brief eligibility-screening questionnaire (Appendix E) were invited to complete the survey. After consenting to participate (Appendix F), respondents completed questions assessing their gendered partner-ideals, gendered partner-perceptions, and relationship 
satisfaction. At the end of the survey, participants provided demographic information and answered a few brief data quality questions (Appendix B).

\section{Data screening}

Six participants (four men and two women) were excluded from analyses because they voluntarily indicated that they provided poor-quality data. Three women and two men were excluded because of a lack of variation in their responses across items and scales (e.g., a participant selected the fourth response option for all items). One man was also excluded for spending less than seven seconds on the entire 47 -item feminine ideals page, clearly demonstrating inattentive responding. For context, the second-fastest male respondent spent only eighteen seconds on the feminine-ideals page, averaging less than half a second per item. While it is likely that inattentive responding negatively impacted the data quality to some extent, there was no clear lower time limit for valid responding because individuals can vary substantially in their reading and reaction speeds. Excluding outliers was an option, but extreme positive skew in the distribution of response times made it difficult to identify a reasonable lower limit using statistics. The speed with which many participants completed the survey suggests a need for an attention screener, which I added into the questionnaire described in Chapter 4.

\section{Results and Discussion}

\section{Hypothesis One}

Hypothesis One examined the factor structure and psychometric properties of the feminine partner-ideals and partner-perceptions measures. Means, standard deviations, internal reliability coefficients, and subscale intercorrelations are reported in Table 4. 
Means were calculated for each individual providing data on at least $80 \%$ of the items in each scale or subscale.

With the exception of dependency/deference and caretaking, all subscales were significantly and positively correlated with each other for both the feminine-ideals and perceptions measures. However, correlations were generally weaker for feminineperceptions than for feminine-ideals. Significant correlation coefficients ranged from .23 to .62 for ideals and from .13 to .39 for perceptions, providing some preliminary evidence of the subscales' convergent validity.

H1a. Five-factor confirmatory models of the feminine partner-ideals and perceptions measures were tested using maximum likelihood estimation in AMOS version 19 structural equation modeling software. The measurement models for ideals and perceptions were identical; only the item wording (i.e., assessing ideals vs. perceptions) was different. In these models, each indicator loaded onto only one of the five factors. Specifically, each item was set to load onto the same factor as the FIS item from which it was adapted. For example, the FIS caretaking item "Women should be gentle" was adapted to "[My partner] is gentle" and "[My ideal partner would] be gentle"; both of these adapted items were set to load on the "caretaking" factor in the perceptions and ideals models, respectively. Each factor was represented by between seven and eleven indicators, depending on the number of items in the original FIS factor model. The variance of each factor was set to one, and latent factors were set to correlate with each other. 
Readers will recall that two items (from the stereotypic images and dependency/deference factors, respectively) were piloted with two potential alternative adaptations. For these items, the adaptation with the higher factor loading on the ideals factor was retained for analyses. It should be noted that one additional change was made between this pilot study and the study described in Chapter 4: item 31 ("spend(s) a lot of time doing her hair and makeup") was changed to "style(s) her hair and wears makeup" to shift the emphasis away from the effort a partner puts into looking stereotypically feminine and onto the result.

Item loadings and factor correlations were examined. Kline (2011) states that standardized factor loadings should ideally be above around .70, meaning that the factor is explaining around half of the variance in the indicator score. However, Preacher and MacCallum (2003) caution against arbitrarily designating a threshold for "large" loadings because loadings are affected by sampling error and the nature of the construct under consideration. Instead, Preacher and MacCallum (2003) recommend examining patterns of loadings. In support of flexibly interpreting the magnitude of standardized loadings, even items on the well-established MRNI-SF have been reported with acceptable loadings as low as .37 (Levant et al., 2015). However, thinking of loadings as high or low can make it easier to understand the overall pattern. Thus, for ease of discussion, I refer to standardized loadings above .60 as "high," those below .40 as "low," and those below .30 as "very low." These determinations are based on the pattern of observed loadings in this sample and should not be considered absolute. 
Loadings for the feminine partner-ideals and -perceptions measures are shown in Table 5. Inspection of item loadings on the feminine-ideals measure revealed a handful of issues, mostly pertaining to the emotionality factor. While some standardized loadings of items on feminine-ideals factors other than emotionality were somewhat low, all were significant and only one was below .30. However, one third of the items in the emotionality factor had nonsignificant loadings. Within this factor, two items (34; "[My ideal partner would] discuss her feelings openly with other women" and item 40; "[My ideal partner would] feel fulfilled by being a relationship]") had negative loadings, and several others had low standardized loadings. Why might the feminine-ideals factor of emotionality have uniquely poor fit?

As discussed in Chapter 2, the emotionality factor of the FIS is distinct in two important ways. First, on a practical level, all the items in the subscale begin with the sentence stem "It is expected that/likely," which could create confusion for participants; they might wonder whether they are being asked to report on their personal ideology or on their impressions of broader societal norms. Items in the subscale also seem less conceptually related to each other than do items in other subscales, including both emotionality in a true sense as well as questions about domesticity, leadership, and some relational attributes. These characteristics raise the possibility that the emotionality factor of the FIS could have emerged as a result of common method variance rather than because the items truly assess a distinct construct, which would represent a threat to measure validity. 
A second attribute that makes the FIS emotionality factor unique is that several of the items pertain to actions or attributes that could be detrimental to personal or relational functioning. For example, "it is expected that a woman who expresses irritation or anger must be going through P.M.S." can certainly reveal beliefs about women's attributes, but I would expect few men to truly want their partner to express anger and irritability. The inclusion of less desirable characteristics is not problematic when assessing femininity ideology, but it does complicate the translation of the items into partner-ideals. Thus, the content of some items were changed dramatically in the creation of the partner-ideal measure (e.g., "It is likely that a woman who gives up custody of her children will not be respected" was adapted to the more directly emotional item "cry if she watched a sad movie"). However, these changes could have created construct drift and thus affected the construct validity of the measure. Most, but not all, of the more "directly" adapted emotionality items had relatively higher standardized factor loadings. A notable exception is item 34, a direct adaptation ("It is expected that women will discuss their feelings openly with one another" to "[My ideal partner] would discuss her feelings openly with other women") that nevertheless had a nonsignificant loading on the emotionality feminine-ideals factor. However, the results of the CFA in Chapter 4, in which the pattern of loadings is different and the emotionality ideals factor is less problematic in general, raises doubts about this explanation.

While most of the obvious problems in the feminine-ideals measure pertained to the emotionality factor, this pattern did not generalize to the feminine-perceptions measure. All but two standardized loadings in the emotionality feminine-perceptions 
factor were below .40 (ranging from .07 to .71 ), but only one loading was nonsignificant. In contrast to the feminine-ideals measure, the stereotypic images and activities factor was the most problematic of the feminine-perceptions measure. Two of the indicators in this factor [item 41; "[My partner] does not do mechanical things" and 45; "[My partner] does not enjoy 'tomboy-type' activities”) had nonsignificant loadings, possibly because they are the only items that focus on specific activities rather than appearance or demeanor. Another item (29; “[My partner] has large breasts") was significant but had a negative loading. The loading's directionality is probably a result of its relation to the two items with the highest loadings on this factor, which involve having a petite body frame and avoiding gaining weight; these three physical characteristics rarely co-occur naturally despite being widely considered desirable (perhaps explaining why the same pattern did not show up for feminine-ideals). One last item (27; "[My partner] does not show anger") had a very low standardized loading.

There were also a couple of problems with the dependency/deference factor. One item (3; "[My partner] is younger than I am") had a nonsignificant loading. Another (item 8; "[My partner] does not initiate sex") had a very low loading. Two other items had low loadings.

Correlations between the latent factors are reported in Table 6. Most feminineideals factors were positively and significantly correlated. Unexpectedly, there were nonsignificant, negative correlations between caretaking and dependency/deference as well as between caretaking and emotionality. While there were no negative correlations between factors in the feminine-perceptions model, only three correlations were 
significant: dependency/deference with purity, dependency/deference with emotionality, and caretaking with emotionality. The small magnitude of several of the correlations in each measure suggests a lack of convergent validity. This is cause for concern because it could mean the factors do not accurately represent the true components of feminineideals and -perceptions.

Next, overall model fit was assessed. The chi-square value was significant for both the feminine partner-ideals measure, $\chi^{2}(935)=2468.38, p<.001$, and the feminine partner-perceptions measure, $\chi^{2}(935)=2968.46, p<.001$, suggesting poor model fit. However, chi-square is sensitive to sample size and a number of other factors unrelated to model validity (Hu \& Bentler, 1999; Kline, 2011; Marsh, Hau, \& Wen, 2004). Thus, alternative fit indices were examined to determine the adequacy of model fit. These indices suggested that model fit was poor for both measures, $\mathrm{CFI}_{\text {ideals }}=.70, \mathrm{RMSEA}_{\text {ideals }}$ $=.07 ; \mathrm{CFI}_{\text {perceptions }}=.54, \mathrm{RMSEA}_{\text {perceptions }}=.08$. According to the standards set by $\mathrm{Hu}$ and Bentler (1999), models with good fit should have a CFI above around .95 and an RMSEA below approximately .06. Others have argued that incremental fit indices above .90 and RMSEA up to .08 indicates reasonable fit (Brown, 2006; Marsh et al., 2004).

The model fit statistics of the feminine measures are discouraging, but these results are perhaps less surprising when considered in context. Readers will recall that the items and hypothesized factors were based on the FIS. However, unlike the MRNISF, the FIS appears to have never been tested using confirmatory factor analysis. Instead, researchers have analyzed the scale's items using principal components analysis (PCA; 
Lehman, 2000; Levant et al., 2007). PCA analyzes all the variance in indicators rather than shared variance and thus results in "components" consisting of correlated indicators rather than latent factors. It is unclear why past researchers have not subjected the FIS to factor analysis, especially because it would allow for the assessment of model fit. An additional failing of using PCA in particular is that it treats components as orthogonal. In contrast, the CFA conducted in H1a allows factors to be correlated. Oblique methods of factor analysis are generally preferable unless there is a substantive reason to believe that factors are uncorrelated (Preacher \& MacCullum, 2003). In any case, these differences in analytic approach make it impossible to compare directly the fit of the created measures in this pilot study to that of the FIS itself. Participants in the final study complete both the FIS and the created feminine measures (see Chapter 4), so a comparison of overall model fit is possible.

It is important to consider why fit indices indicate poor model fit. Specific areas of poor fit have already been identified (i.e., negative, low, and nonsignificant factor loadings). However, there are several other possible reasons why the fit indices might be lower than acceptable. One such reason does not relate to the validity of the measure at all; instead, it has to do with the way that alternative fit indices are calculated. "Fit indices" truly reflect model misfit, or an aggregate of discrepancies between the expected and observed data. This fact is reflected in the influence of sample size on the model chisquare value: because model misfit is cumulative, more cases results in more total potential misfit. Alternative fit indices are all based on the chi-square value, although different categories of fit indices adjust for different factors such as sample size and 
model parsimony (Kline, 2011). Parsimony is particularly relevant to the models examined in $\mathrm{H} 2 \mathrm{a}$. Each factor has between seven and eleven indicators, and there are a total of 45 indicators. This represents a relatively large model. Unfortunately, while the ratio of indicators to factor has no bearing on measurement validity, it can result in biased estimates of the overall model fit (e.g., Marsh, Balla, \& Grayson, 2005; Marsh et al., 2004). Marsh et al. (2004) argue that conventional guidelines for fit indices are too restrictive to apply to certain measures with multiple factors:

It is my experience that it is almost impossible to get an acceptable fit (e.g., CFI, RNI, TLI > .9; RMSEA < .05) for even "good" multifactor rating instruments when analyses are done at the item level [versus parceling, for example] and there are multiple factors (e.g., 5-10), each measured with a reasonable number of items (e.g., at least 5-10/per scale) so that there are at least 50 items overall (p. 325). Marsh et al. (2004) added that researchers on a large, unnamed SEM listserv were unable to provide a published counterexample of a large measure that would be deemed "acceptable" based on its fit indices. Nevertheless, many respondents to the query expressed reluctance to modify guidelines based on such practical concerns. While Marsh et al. (2004) caution against an overreliance on absolute cutoffs for fit indices when considering a model in isolation—especially a large model—-they do note that such indices are useful for comparing alternative models. When comparing models, it is important to consider whether parsimony is a valuable goal as long as a model is simple enough to realistically interpret (Marsh et al., 2005); the ultimate objective of choosing a 
model should be to identify the one that most closely approximates the truth by accurately representing the underlying constructs and their interrelationships.

Interestingly, the impact of the number of items in a model can have different effects depending on the outcome under consideration; Kenny and McCoach (2003) note that increasing the number of items in a correctly-specified model tends to improve absolute fit indices (such as RMSEA) but reduce CFI values. This trend could explain why RMSEA values of the feminine measures were close to acceptability whereas CFI values were quite low, based on the guidelines advanced by Hu and Bentler (1999).

Of course, it is also possible that the poor fit indices reflect a real problem with the conceptualization or measurement of the constructs. Perhaps the dimensions of the FIS do not match the gendered domains in which men hold ideals or evaluate their female partners. This project used a deductive process to construct measures based on a body of literature examining dimensions of masculinity and femininity. The decision to use this approach was primarily based on resource constraints rather than a belief that it would produce the most valid measures. It would be informative to create measures of feminine partner-ideals and -perceptions using a deductive approach. To the best of my knowledge, only one published study has utilized this approach. Lee et al.'s (2000) work was discussed in Chapter 1 of this document, and it was noted that American men prescribed the dimensions of warmth, traditional femininity, attractiveness, and strength while proscribing abusiveness, femininity, unattractiveness, and nontraditionality. However, it is important to note that ideals might have shifted in the nearly two decades since that study was published. 
Setting aside the methodological problems with the study, it is notable that there are both areas of convergence and divergence with the dimensions of the FIS. Several of the individual qualities that Lee et al. (2000) found men desired (e.g., "Good homemaker," "Good with kids," "Caring," "[Not] vulgar") closely echo constructs included on the FIS. Conversely, some qualities specifically opposed those of the FIS, almost entirely in regards to dependency/deference (e.g., "[Not] dependent," "Assertive"). Other characteristics, like sexual fidelity, are entirely absent. Some of the divergence between the dimensions captured by the FIS and participants of Lee et al.'s (2000) study could be a result of the latter's deductive approach. One weakness of the deductive approach is that it assumes a degree of self-awareness and cultural perspective that the average participant might not possess. For example, certain romantic relationship norms such as sexual fidelity might be so ingrained as to be assumed by the majority of participants. Assuming this were true, then few participants might spontaneously report sexual fidelity as a norm (it is merely implicit in their relationship expectations); these same participants might report a high desire for their romantic partner to be sexually faithful if asked about it directly. There is clearly value to both deductive and inductive approaches. The discrepancies between the dimensions of the FIS and those found in Lee et al. (2000), combined with the poor model fit of the feminine measures created for the present study, all point to a need for more research on the structure of femininity in general and on feminine partner-ideals in particular.

Returning to a discussion of the created feminine measures, it is also possible that the dimensions do, in fact, mirror those of femininity ideology. If this were true, the 
model misfit could indicate the failure of the adapted items to adequately reflect the ideas assessed by the FIS. In Chapter 2, I first discussed how some items were more difficult to adapt than others. For example, while "Women should have soft voices" is reasonably adapted to "[My ideal partner would] have a soft voice," an item such as "A girl should be taught how to catch a husband" has no obvious partner-ideal or -perception equivalent.

Ultimately, it is unclear whether the problem primarily lies in the dimensions themselves, in the adaptation of certain items, or in a statistical artifact of the way alternative fit indices are calculated. The CFA in Chapter 4 provides more insight into the plausibility of some of these explanations. Additionally, Chapter 4 explores some plausible alternative factor models (e.g., including a second-order factor, adding a general feminine ideals/perceptions factor).

H1b. While Cronbach's alpha is typically used to assess the internal consistency of scales, it assumes that items loading on each factor have tau equivalence (Zinbarg, Revelle, Yovel, \& Li, 2005). However, this assumption was not supported by the factor analysis discussed in the previous section. For measures that fail to meet this assumption, Cronbach's alpha is a biased measure of internal consistency (Dunn, Baguley, \& Brunsden, 2013; Zinbarg et al., 2005). Thus, McDonald's omega (McDonald, 1999), which relaxes this assumption, was used. Because the base SPSS package cannot compute omega, it was calculated in the open-source software program $R$ version 3.3.2 using code created by Dunn et al. (2013).

While omegas for the total scales were good (.90 for ideals and .83 for perceptions), omegas for the subscales ranged widely (see Table 6). For the feminine- 
ideals subscales, omegas ranged from .60 to .88 . Reliability was below acceptable for both emotionality (.60) and caretaking (.69). For transparency, Cronbach's alphas were all within .01 of the equivalent omega. Three of the five feminine-perceptions subscales (i.e., stereotypic images and activities, dependency/deference, and emotionality) had below-acceptable omegas. However, omega for dependency/deference was close to acceptable at .69. With one exception, Cronbach's alphas for the feminine partnerperceptions subscales were the same or up to .08 lower than the equivalent partner-ideals, ranging from .43 to .80 .

\section{Hypothesis Two}

Hypothesis Two examined the factor structure and psychometric properties of the masculine partner-ideals and partner-perceptions measures. Means, standard deviations, internal reliability coefficients, and subscale correlations are reported in Table 7. Means were calculated for each participant providing data on at least $80 \%$ of the items in each scale or subscale.

With the exception of self-reliance and restrictive emotionality, all bivariate correlations between masculine ideals subscales were significant and positive, with significant coefficients ranging from .16 to .54 . Significant masculine-perceptions subscale correlation coefficients ranged from .14 to .42 , although there were four nonsignificant correlations. This general pattern provides preliminary evidence of the subscales' convergent and discriminant validity.

H2a. Seven-factor confirmatory models of the masculine partner-ideals and perceptions measures were tested using maximum likelihood estimation in AMOS 
version 19 structural equation modeling software. As with the feminine measures, identical measurement models were tested for masculine-ideals and -perceptions. Each item was set to load onto the same factor as the MRNI-SF item from which it was adapted. The variance of each factor was set to one, and latent factors were allowed to correlate with each other. Readers will recall that five items were piloted with two potential alternative adaptations. For these items, the adaptation with the higher standardized loading on its respective ideals factor was retained for the final version of the measure. (For the Toughness factor, item 17 was replaced by the alternative adaptation of item 20 , which had a higher loading.) Thus, each factor was represented by exactly three indicators.

First, measure properties were examined in closer detail. Inspection of item loadings on both the masculine-ideals and -perceptions measures revealed that all loadings were significant, and most standardized loadings were high (ranging from .49 to .97 for ideals and .44 to .96 for perceptions). Item loadings for the masculine partnerideals and -perceptions measures are shown in Table 8.

While it did not seem to result in poor fit, I feel it is important to note that all of the items on the original dominance subscale were contextualized as leadership or status in the family domain, whereas the original items focus on men's natural role as leaders. For example, the original item "The President of the US should always be a man" was adapted to "[My partner] holds a high-status job." While I would not necessarily characterize this reframing as bad for the validity of the measure, it could be interpreted as construct drift from the original MRNI-SF. It remains to be seen whether this change 
is reflected in the correlations between the original MRNI-SF subscale and the masculine-ideals one. This question is explored in Chapter 4.

For the masculine-ideals measure, all but one correlation between latent factors was significant (the exception was self-reliance through mechanical skills and restrictive emotionality). Most correlations were of small-to-moderate magnitude, supporting convergent validity. Correlations on the masculine-perceptions measure were smaller than for ideals, on average. Six factor correlations were nonsignificant (four of which were with the restrictive emotionality factor), and most were small to very small. It is unclear why these correlations were smaller than for the masculine-ideals factors. Correlations between the latent factors are reported in Table 9.

Next, overall model fit was assessed. The chi-square value was significant for both the masculine partner-ideals measure, $\chi^{2}(168)=324.95, p<.001$, and the masculine partner-perceptions measure, $\chi^{2}(168)=298.95, p<.001$, suggesting poor model fit. However, alternative fit indices indicated that model fit was acceptable to good, $\mathrm{CFI}_{\text {ideals }}$ $=.94, \mathrm{RMSEA}_{\text {ideals }}=.06 ; \mathrm{CFI}_{\text {perceptions }}=.95, \mathrm{RMSEA}_{\text {perceptions }}=05$.

There is one minor difference between the factor structure of the MRNI-SF as analyzed by Levant et al. (2013) and the created measures: the MRNI-SF includes an additional latent factor, general traditional masculinity, that is uncorrelated with the seven specific male role domains. In this model, each indicator loads on both this general factor and one specific domain of masculinity. Thus, it is possible that while fit was good for the hypothesized model, a related alternative model could have even better fitsuggesting that it is a more accurate representation of the true relationships between 
factors. In Chapter 4, this alternative model and some other plausible options are explored and compared.

H2b. It was hypothesized that internal consistency coefficients would be acceptable $(\geq .70)$ for each of the hypothesized factors contained within the masculine partner-ideals and partner-perceptions measures. McDonald's omega was excellent for the total masculine ideals scale (.88) and acceptable for the total masculine perceptions scale (.75). For masculine partner-ideals, omegas ranged from .70 to .90 . Thus, all reliabilities were acceptable to excellent. Omegas for the subscales in each measure are shown in Table 9. For the sake of transparency, Cronbach's alphas for the subscales ranged from .67 to .90 .

Omegas for the masculine partner-perceptions subscales were slightly below the predetermined cutoff of acceptability for both avoidance of femininity (.67) and toughness (.68). However, all other subscales had good to excellent reliabilities. Cronbach's alphas ranged from .67 to .92 .

For both women and men, reliabilities for the partner-perceptions measures were expected to be lower than for partner-ideals. While partner-ideals are presumably consistent across situations and facets, a partner's fulfillment of specific facets of gender roles is likely influenced by a variety of both personal and situational factors. For example, the masculine avoidance of femininity subscale has two items about preferring stereotypically masculine to stereotypically feminine media, but it also has an item about not wearing makeup. A male partner's fulfillment of these different facets of avoidance of femininity could be driven by a variety of factors including his personal media tastes, 
his desire to look a particular way, and the norms of his peer group. Past work on partner-ideal discrepancies has focused on the structure of the ideal scales rather than the corresponding perceptions scales, but some research suggests that dimensions of partnerperceptions have yielded lower internal consistencies than the corresponding partnerideals (e.g., Eastwick \& Neff, 2012), even when the ideals and perceptions being measured were single-word traits (e.g., communicative, affectionate) rather than specific roles.

As just discussed, reliability was acceptable to excellent for the both the aggregate masculine-ideals and -perceptions measures and nearly all of the subscales. However, low reliability on partner-perceptions subscales could negatively impact the statistical power of the test of the correlations between various gendered attributes and IPV perpetration. If the same pattern of slightly lower-than-acceptable reliabilities is found in some of the partner-perceptions subscales in the final study sample, it will be noted as a limitation.

\section{Hypothesis Three}

The mean correlation between women's masculine partner-ideals and perceptions was $.51(S D=.30$, range -.38 to .95$)$, showing that most women reported relatively high consistency between gendered ideals and perceptions. Additionally, PRQC scores $(M=5.74, S D=1.15)$ suggested that women were generally satisfied with their relationships. As is common in measures of relationship satisfaction, the distribution of PRQC scores was somewhat negatively skewed (-1.14). 
The mean correlation between men's masculine partner-ideals and -perceptions was $.39(S D=.28$, range -.35 to .92$)$, showing that most men reported moderate consistency between their gendered ideals and perceptions. PRQC scores $(M=5.69, S D$ $=1.14)$ suggested that men were generally satisfied with their relationships. The distribution of PRQC scores was somewhat negatively skewed (-1.20).

Hypothesis 3 states that gendered partner-ideal discrepancies will be negatively associated with relationship satisfaction. These hypotheses were tested by calculating the correlation between gendered partner-ideal discrepancies (i.e., negative one multiplied by the within-person correlation between ideals and perceptions) and PRQC scores.

H3a. Women's masculine partner-ideal discrepancies negatively correlated with PRQC scores, $r(260)=-.60, p<.001$.

H3b. Similarly, men's feminine partner-ideal discrepancies negatively correlated with PRQC scores, $r(355)=-.60, p<.001$.

Generally, Hypothesis 3 confirms the results of the pilot study described in Chapter 2: gendered partner-ideal discrepancies are negatively correlated with relationship satisfaction. Put another way, greater consistency between gendered partnerideals and partner-perceptions predicts higher relationship satisfaction. However, concerns about the validity of the feminine-ideals and -perceptions measures make these conclusions tentative for male participants. The validity of the feminine-ideals and perceptions measures are explored in greater depth in Chapter 4 . 
Chapter 4. The Present Study: Associations between Gendered Partner-ideal

\section{Discrepancies, Relationship Satisfaction, and IPV}

\section{Overview}

This study examines the associations between gendered partner-ideal discrepancies, relationship satisfaction, and IPV. Specifically, gendered partner-ideal discrepancies were expected to predict IPV perpetration (including both physical violence and emotionally controlling behaviors), mediated by the negative association between ideal discrepancies and relationship satisfaction. Traditional partner-gender ideology was explored as a potential predictor of gendered partner-ideals. Additionally, conformity to specific gender role norms (based on partner-perceptions) was examined as a predictor of IPV perpetration.

This project involved the collection of cross-sectional data from heterosexual romantic partners using an online questionnaire. While participants were invited to provide contact information for their partners so that we could collect data from both partners, the resulting dyadic data is not analyzed in this manuscript. All participants provided data regarding their endorsement of traditional partner-gender ideology (i.e., ideology regarding the gender of the participant's romantic partner), their gendered partner-ideals, gendered partner-perceptions, relationship satisfaction, and IPV perpetration and victimization.

\section{Hypotheses}

The following hypotheses were tested: 
Hypothesis One (H1). An individual's partner-gender ideology predicts their gendered partner-ideals (e.g., to the extent that an individual thinks men should be unemotional, they will also desire that characteristic in their romantic partner). Specifically:

H1a. Aggregate feminine gender ideology (i.e., the average endorsement of all subdimensions) positively correlates with aggregate feminine partner-ideal scores.

H1b. Each dimension of feminine gender ideology positively correlates with the corresponding feminine partner-ideal dimension.

H1c. Aggregate masculine gender ideology positively correlates with aggregate masculine partner-ideal scores.

H1d. Each dimension of masculine gender ideology positively correlates with the corresponding masculine partner-ideal dimension.

Hypothesis 2 (H2). For both men and women, aggregate gendered partner-ideal discrepancies positively predict physical IPV and emotional control perpetration, mediated by relationship satisfaction. Specifically, gendered partner-ideal discrepancies negatively predicts relationship satisfaction. In turn, lower relationship satisfaction predicts greater physical IPV and emotional abuse perpetration. While no specific gender differences in this mediation model were hypothesized, I planned to run a moderated mediation with gender as the moderator (see Figures 1 and 2). Using moderated mediation rather than testing separate mediation models for men and women will increase the statistical power of the analysis and allow for the detection of different effects in men and women (Edwards \& Lambert, 2007; Hayes, 2013). I proposed to test Hypothesis 2 
using Model 59 of the PROCESS macro for SPSS (Hayes, 2012; Hayes, 2013), with gendered partner-ideal discrepancies as the independent variable (X), relationship satisfaction as the mediator (M), IPV as the outcome variable (Y), and gender as the moderator (W). Models were tested separately for each component of physical abuse (see Figure 1) and for emotional abuse (see Figure 2).

H2a. Gendered partner-ideal discrepancies positively predict physical IPV perpetration (modeled separately for physical assault and injury), mediated by relationship satisfaction. Specifically, the total and the indirect effects of partner-ideal discrepancies on IPV (path c' in Figure 1) are significant and positive, while the paths between partner-ideal discrepancies and relationship satisfaction (path a) and between relationship satisfaction and physical IPV (path b) are negative. There were no specific hypotheses about gender moderating any of the direct or indirect effects, although some past research suggests that the direct effect of ideal discrepancies on physical IPV perpetration may exist only in men (Jaspaert, 2015). Additionally, while one study found that there was a stronger relationship between relationship satisfaction and physical IPV perpetration among men than among women (Stith et al., 2008), other research has not found this difference (Jaspaert, 2015).

H2b. Gendered partner-ideal discrepancies positively predict emotional abuse perpetration, mediated by relationship satisfaction (see Figure 2). Specifically, the total and the indirect effects of partner-ideal discrepancies on IPV (path c' in Figure 1) are significant and positive, while the paths between partner-ideal 
discrepancies and relationship satisfaction (path a) and between relationship satisfaction and emotional IPV (path b) are negative. Again, there were no specific hypotheses about gender moderating any of the direct or indirect effects, although Jaspaert (2015) suggests that the direct effect of ideal discrepancies on psychological IPV perpetration exists only in men.

Hypothesis 3 (H3). Partner-perceptions of gender role conformity predict both physical IPV and emotional control. Specifically:

H3a. Women's aggregate masculine partner-perceptions positively correlate with their reports of IPV victimization (for both physical IPV and emotional control).

H3b. Women's masculine partner-perceptions for each domain of masculinity positively correlate with women's reports of physical IPV and emotional control victimization.

H3c. Men's aggregate feminine partner-perceptions negatively correlate with their reports of IPV victimization (for both physical IPV and emotional control).

H3d. Men's feminine partner-perceptions for each domain of femininity negatively correlate with men's reports of physical IPV and emotional control victimization.

\section{A Priori Power Analysis and Sample Size Justification.}

A priori power analyses were conducted to estimate the sample sizes needed to detect significant results for each hypothesis.

$\mathrm{G}^{*}$ Power was used to determine an adequate sample size to detect significant effects for $\mathrm{H} 1$ at alpha $=.05$ and power $=.80$ (Erdfelder, Buchner, Faul, \& Brandt, 2004). Estimates of effect sizes were obtained from the original small pilot study conducted with 
an undergraduate population. Correlations between domains of gender ideology and corresponding gendered partner-ideals ranged from .18 to .62 in the pilot study, indicating that a sample size of 234 participants of each gender were needed.

Power analyses for H2 were conducted using MedPower (Kenny, 2017), an online application that calculates the sample size necessary to detect each effect in a mediation given estimates of paths a, b, and c' at a specified alpha and level of power. This model has two main differences from the one tested by H2. First, it estimates regular mediation rather than moderated mediation. However, past research provides no basis for an estimate of moderated effects. Second, MedPower uses the joint significance method for the indirect effect (i.e., determining the "significance" of the indirect effect based on whether both paths $\mathrm{a}$ and $\mathrm{b}$ are significant) rather than the bootstrapping method utilized by PROCESS. However, bootstrapping and the test of joint significance results in comparable conclusions a majority of the time (Hayes \& Scharkow, 2013). Biascorrected bootstrapping, which I used to test $\mathrm{H} 2$, has particularly high power to detect indirect effects (Preacher et al., 2007); thus, MedPower estimates of the required sample may be biased upward.

As previously discussed, Jaspaert and Vervaeke (2014) is the only published study exploring the relationship between ideal discrepancies, relationship satisfaction, and IPV (including both the physical and psychological violence perpetration subscales of the CTS2). To run the a priori power analysis for $\mathrm{H} 2$, estimates of effect sizes were taken from Jaspaert (2015), which has more complete information on the same data than is available in Jaspaert and Vervaeke's (2014) published study. 
However, there are differences between the present study and Jaspaert (2015) that could decrease the applicability of the effect size estimates. Jaspaert (2015) had a different sample (i.e., Dutch-speaking heterosexual couples) and used different measures of ideal discrepancies, relationship satisfaction, and psychological IPV (although the measure of physical IPV was the same). There are also analytical differences between Jaspaert (2015) and the present study. Rather than using simple mediation or moderated mediation, Jaspaert (2015) fits the data to mediated actor-partner interdependence models. These models include both effects within a person (i.e., the relationships between an individual's ideal discrepancies, relationship satisfaction, and IPV perpetration) and between romantic partners in a couple (e.g., the relationship between one person's relationship satisfaction and the other's IPV perpetration). These dyadic models showed that individuals' ideal discrepancies consistently predicted their partners' relationship satisfaction between $\beta=-.23$ to -.27 . Additionally, estimates in these models were adjusted for social desirability biases in responding.

Given these analytical differences, the standardized beta coefficients from Jaspaert (2015) are not equivalent to those that would be obtained from regular mediation. However, they can be used to provide a rough estimate of the parameters in a regular mediation model. Beta coefficients from the dyadic models were averaged between the genders to come up with an estimate of the equivalent $\mathrm{a}, \mathrm{b}$, and c' paths in a regular mediation model. For psychological IPV perpetration, path a was estimated at -.45 , path $\mathrm{b}$ at -.16 , and path c' at .23 . Entered into MedPower with alpha $=.05$ and power $=.80, \mathrm{a}$ projected sample size of $n=171$ would be necessary to detect the direct effect of ideal 
discrepancies on IPV perpetration and $n=347$ to detect the indirect effect through relationship satisfaction. For physical IPV perpetration, path a was estimated at -.45, path $\mathrm{b}$ at -.13 , and path $\mathrm{c}^{\prime}$ at .15 . With alpha $=.05$ and power $=.80$, a projected sample size of $n=418$ would be necessary to detect the direct effect and $n=555$ to detect the indirect effect. Because moderation adds complexity to the mediation model, it would be prudent to sample more participants than the highest of these estimates, or at least $n=$ 300 participants of each gender.

For $\mathrm{H} 3 \mathrm{a}$ and $\mathrm{H} 3 \mathrm{~b}, \mathrm{G} *$ Power was used to estimate the projected sample size needed to detect significant effects at alpha $=.05$ and power $=.80$ (Erdfelder et al., 2004). Past research has found that men's masculine role conformity has a small to medium correlation with intimate partner violence and aggression (around $r=.20$ to .30 ; Kearney, 2015; Reidy et al., 2014; Sears, 2011; Tager et al., 2010). Readers will recall that data on men's masculinity are obtained from their female partners. Thus, it was determined that a sample size of $n=193$ women would be sufficient for H3a and H3b.

It was more difficult to find estimates for the power analysis of $\mathrm{H} 3 \mathrm{c}$ and $\mathrm{H} 3 \mathrm{~d}$. As discussed in the literature review, there are no published studies on women's feminine role conformity and IPV perpetration. Thus, I relied upon estimates of related constructs: Reidy et al. (2009) found that women's feminine role conformity correlates with physical and verbal aggressiveness around .20, and Kearney (2015) found that correlations between individual domains of women's feminine role conformity and attitudes toward women's dating violence range from .02 to -.30 . To detect a relatively small correlation 
of $r=.15$ at alpha $=.05$ and power $=.80$, a sample size of $n=346$ men reporting on their female partners' perceived feminine attributes was expected to suffice.

Past research on studies of IPV within romantic relationships have reported removing around $8-18 \%$ of the sample because of missing data (Riggs \& O'Leary, 1996; Riggs, O’Leary, \& Breslin, 1990), although the threshold of missing data (i.e., any missingness versus a certain percentage of missing data points) was not specified in these studies. Building an extra $18 \%$ into the estimated $n=555$ participants required to detect the indirect effects modeled in Hypothesis 2 gives a total sample size of $n=677$ (677 $.18^{*} 667=555$ ). Dividing this sample equally by gender results in $n=339$ men and $n=$ 339 women. However, $n=339$ men could be too few for an adequately powered test of $\mathrm{H} 3 \mathrm{c}$, as discussed in the previous paragraph. Increasing the estimated $n=346$ men needed to test $\mathrm{H} 3 \mathrm{c}$ by $18 \%$ results in $n=409$. Thus, I proposed to collect data from $n=$ 409 men and $n=339$ women.

\section{Method}

\section{Participants}

Eligible participants were cisgender (i.e., identifying with the gender of their birth) English-speaking members of heterosexual couples living in the United States who had been in a romantic relationship for at least six months and were each at least 18 years old. Based on previous research recruiting through MTurk, recruited participants were expected to be, on average, in their early 30 s, majority White, and have a few years of post-secondary education (Berinsky, Huber, \& Lenz, 2012). Respondents recruited through MTurk tend to be somewhat younger than nationally stratified samples (Huff \& 
Tingley, 2015). The final sample for the present study consisted of $n=344$ women and $n$ $=406$ men. Sample characteristics are reported separately by gender because men and women completed different measures.

Female participants ranged from nineteen to 72 years old $(M=36.2, S D=10.5$, Med. $=34)$. The majority $(89.5 \%)$ described themselves as straight or heterosexual, $9.3 \%$ as bisexual, and $1.2 \%$ as Other (including variants of bi- and pansexuality). The majority (76.7\%) was White, followed by Black or African American (8.4\%), Asian (4.7\%), Latino or Hispanic (2.3\%), and Native American (.6\%); $7.3 \%$ reported two or more races/ethnicities. Frequencies of partner races were similar; $71.5 \%$ reported that their partner was White, followed by Black or African American (10.5\%), Latino or Hispanic (4.9\%), Asian (4.9\%), Native American (.9\%), Other (.9\%), and Pacific Islander (.6\%); with $5.8 \%$ reporting two or more categories.

The highest level of education obtained by female participants varied widely, with $.3 \%$ having some high school or less, $30.2 \%$ having a high school diploma or GED, 23.0\% holding an Associate's or occupational degree, 32.6\% holding a Bachelor's, $10.5 \%$ holding a Master's, $2.6 \%$ holding a professional school degree, and $.9 \%$ with a doctoral degree. Reported annual personal income (assessed separately from the partner's income) ranged from $\$ 0-225,000(M=\$ 29,486.99, S D=29,957.21$, Med $=$ 25,0000; these numbers exclude one participant, who reported that she and her partner each earned an implausible $\$ 15$ million annually). Regarding occupation, $74.7 \%$ were employed part- or full-time, $7.0 \%$ were students, and $21.5 \%$ were neither employed nor students (these numbers add to more than $100 \%$ because $3.2 \%$ were employed students). 
Of women, $69.5 \%$ were married or in a domestic partnership and the other $30.5 \%$ were in a romantic relationship. The length of these participants' relationships ranged from six months to 45.4 years $(M=10.7$ years, $S D=7.3, \mathrm{Med}=9.7)$. Those who were married or in a domestic partnership had been married or domestically partnered for an average of 10.9 years $(S D=8.3, \mathrm{Med}=10.1)$. Eighty-eight percent currently lived with their romantic partner, while the other twelve percent did not. The majority $(68.3 \%)$ had one or more child, and $59.0 \%$ lived with one or more children under 18 . The majority of female participants $(88.4 \%)$ reported taking the survey alone, while $10.2 \%$ said their partner was nearby but couldn't see how they answered, and $1.5 \%$ said their partner was nearby and could see how they answered.

Male participants were, on average, slightly older than female participants, ranging from 19 to 75 years old $(M=37.8, S D=12.1$, Med.$=34)$. The majority $(97.0 \%)$ described themselves as straight or heterosexual, $2.5 \%$ as bisexual, and .5\% as Other. The majority (72.7\%) was White, followed by Asian (6.9\%), Latino or Hispanic (6.9\%), Black or African American (4.9\%), Native American (.7\%), and Other (.7\%); while 7.1\% reported two or more categories. Frequencies of partner race/ethnicity were similar; 69.5\% reported that their partner was White, followed by Asian (11.6\%), Latino or Hispanic (8.6\%), Black or African American (4.2\%), Native American (.7\%), Pacific Islander $(.5 \%)$, and Other $(.5 \%)$; with $4.4 \%$ reporting two or more categories.

The highest level of education obtained by male participants varied, with .2\% having some high school or less, $22.2 \%$ having a high school diploma or GED, 15.8\% holding an Associate's or occupational degree, 41.1\% holding a Bachelor's, 15.0\% 
holding a Master's, 3.0\% holding a professional school degree, and 2.7\% holding a doctoral degree. Reported annual personal income ranged from $\$ 0-700,000(M=$ $\$ 34,985.41, S D=44,038.77, \mathrm{Med}=\$ 30,000)$. Regarding occupation, $75.7 \%$ were employed part- or full-time, $7.2 \%$ were students, and $20.2 \%$ were neither employed nor students (percentages add to more than 100\% because 3.0\% were employed students).

Among male participants, $65.8 \%$ were married or in a domestic partnership and the other $34.2 \%$ were in a romantic relationship. Reported relationship lengths ranged from six months to 54.8 years $(M=10.2$ years, $S D=10.4$, Med $=6.7)$. Men who were married or in a domestic partnership had been married or domestically partnered for an average of 11.2 years $(S D=11.5, \mathrm{Med}=7.3)$. A large majority $(82.5 \%)$ currently lived with their romantic partner, while the other $17.5 \%$ percent did not. Just under half $(49.0 \%)$ had no children, and less than half (42.6\%) lived with one or more children under 18 . The majority of male participants $(85.0 \%)$ reported taking the survey alone, while $13.3 \%$ said their partner was nearby but couldn't see how they answered. One percent said their partner was nearby and could see how they answered.

\section{Measures}

Partner-gender ideology. Participants completed measures assessing their ideology regarding the gender of their partner. Because only participants in heterosexual relationships were eligible to participate, men completed a measure of femininity ideology and women a measure of masculinity ideology.

Femininity ideology. Male participants completed the 45-item Femininity Ideology Scale (FIS; Lehman, 2000; Levant et al., 2007), which asks respondents to 
indicate their disagreement or agreement with each item on a five-point scale from $1=$ Strongly disagree to $5=$ Strongly agree. The FIS has five subscales: stereotypic image and activities (e.g., "Girls should not enjoy 'tomboy-type' activities"), dependence/deference (e.g., "A woman should not make more money than her partner"), purity (e.g., "A woman should remain a virgin until she is married"), caretaking (e.g., "A woman should be responsible for teaching family values to her children"), and emotionality (e.g., "It is expected that women will have a hard time handling stress without getting emotional").

The FIS has good convergent and discriminant validity with measures of gender role conformity, gender role conflict, and feminist identity development (Lehman, 2000; Levant et al., 2007). Men tend to have stronger endorsement of traditional femininity ideology than do women, echoing findings on masculinity ideology and measures of egalitarianism (Levant et al., 2007). Cronbach's alpha for the subscales range from .80 to .89, with an alpha of .93 for the total scale (Levant et al., 2007). Cronbach's alpha for the present study is reported in Table 10. Alpha for the aggregate scale was .97 and ranged from .87 to .95 for each of the subscales.

Masculinity ideology. Female participants completed the short form of the Male Role Norms Inventory (MRNI-SF; Levant et al., 2013), a 21-item scale measuring endorsement of traditional masculinity ideology. The MRNI-SF consists of seven subscales: avoidance of femininity (e.g., "Men should watch football games instead of soap operas"), negativity toward sexual minorities (e.g., "Homosexuals should never kiss in public"), self-reliance through mechanical skills (e.g., "Men should have home 
improvement skills"), toughness (e.g., "When the going gets tough, men should get tough"), dominance (e.g., "A man should always be the boss"), importance of sex (e.g., "A man should not turn down sex"), and restrictive emotionality (e.g., "A man should never admit when others hurt his feelings"). General masculinity ideology is calculated as the mean of all items. Respondents are asked to indicate their level of agreement with each item on a scale ranging from $1=$ Strongly disagree to $7=$ Strongly agree

Both the total MRNI-SF and its subscales have demonstrated good convergent and discriminant validity with measures of gender role conflict, gender role conformity, and an early measure of masculinity ideology (Levant et al., 2013; Levant, Hall, Weigold, \& McCurdy, 2016). In previous research with female participants, the subscales of the MRNI-SF have had Cronbach's alphas ranging from .75 to .88 , with .94 for the total score (Levant et al., 2013). Alphas for the study sample are reported in Table 11. Alpha for the aggregate scale was .94 and ranged from .75 to .94 for each of the subscales.

\section{Gendered partner-ideals, partner-perceptions, and ideal discrepancies.}

Feminine partner-ideals, partner-perceptions, and ideal discrepancies. The creation of the feminine partner-ideals and partner-perceptions measures is described in Chapters 2 and 3 (see Appendix A). Both measures contain a total of 45 items and are intended to assess the feminine role norms of stereotypic images and activities, dependency/deference, purity, caretaking, and emotionality. For the feminine partnerideals measure, male participants are asked to consider how they would personally like their romantic partner to be and to rate each item on a scale from $1=$ strongly disagree to 
$5=$ strongly agree. For feminine partner-perceptions, men are asked to rate how well each item describes their current partner on the same scale.

Aggregate feminine partner-ideals and -perceptions are calculated as the mean of the items in the respective scale. Feminine partner-ideals and-perceptions for each subscale are calculated as the mean of the items contained within. Descriptive statistics and internal consistency coefficients are provided in Table 12. The factor structure of this measure is explored in the Results section of this chapter.

The results of the pilot study for this measure discussed in Chapter 3 revealed that 1) that there was little support for the hypothesized factor structures of feminine partnerideals or -perceptions, and 2) several of the hypothesized feminine-ideals and perceptions subscales showed unacceptably low internal reliability. However, the total scales had acceptable internal reliability. Together, these results raise doubts about the validity of these measures. It is inadvisable to make conclusions based on potentially faulty measures. For this reason, I decided that testing the hypotheses in this chapter that rely upon the feminine partner-ideals and -perceptions measures would be contingent on the results of the CFAs of those measures. If a CFA supported the originally hypothesized five-factor structure, I planned to compute and report tests of all original hypotheses. Otherwise, I would not test the hypotheses in question.

Masculine partner-ideals, partner-perceptions, and ideal discrepancies. The process of creating the masculine partner-ideal and partner-perceptions measures is described in Chapters 2 and 3 (see Appendix A). Both measures contain a total of 21 items and are intended to assess the masculine role norms of avoidance of femininity, 
negativity toward sexuality minorities, self-reliance through mechanical skills, toughness, dominance, importance of sex, and restrictive emotionality. For the masculine partnerideals measure, female participants are asked to consider how they would personally like their romantic partner to be and to rate each item on a scale from $1=$ Strongly disagree to $7=$ Strongly agree. For masculine partner-perceptions, women are asked to rate how well each item describes their current partner on the same scale.

Aggregate masculine partner-ideals and perceptions are calculated as the mean of the items in each respective scale. Masculine partner-ideals and-perceptions for each domain are also calculated as the mean of the items contained within each subscale, pending the factor analysis described in the Results section. Descriptive statistics and internal consistency coefficients are provided in Table 13. McDonald's omega was good for both the aggregate ideals and perceptions measures. Internal consistency coefficients for the subscales ranged from .76 to .93 for ideals and from .63 to .91 for perceptions. Coefficients for the avoidance of femininity and toughness perceptions subscales were lower than acceptable (i.e., below .70); this may impact the statistical power of the relevant tests in $\mathrm{H} 3 \mathrm{~b}$. A confirmatory factor analysis of this measure is described in the Results section of this chapter.

Masculine partner-ideal discrepancies are calculated as described in Chapters 2 and 3 (i.e., within-subjects correlations between masculine-ideals and masculineperceptions, multiplied by -1). On average, women in the present sample perceived small-to-moderate masculine-ideal discrepancies $(M=-.48, S D=.32$, Min $=-.97$, Max 
$=.60$ ). Scores could not be computed for two women who responded to all masculineideals scores with the same response option.

Relationship satisfaction. Relationship satisfaction was assessed using the Perceived Relationship Quality Components Inventory (PRQC; Fletcher, Simpson, \& Thomas, 2000b). This measure was described in greater detail in Chapter 3. Descriptive statistics and internal consistency coefficients in the present sample are given in Table 14. Cronbach's alpha of the aggregate scale was very high in the present sample at .97 for both women and men. For women, subscale reliabilities ranged from .92 to .95 ; for men, they ranged from .93 to .98 . Generally, both women and men reported being satisfied with their relationship $\left(M_{\text {women }}=5.78, S D_{\text {women }}=1.24 ; M_{\text {men }}=5.90, S D_{\text {men }}=1.04\right)$.

IPV. As described in Chapter 1, the revised Conflict Tactics Scale (CTS2) and similar behavioral frequency measures are the most popular measures of IPV. Because the CTS and CTS2 are so frequently used, they offer the advantage of comparability to a large body of existing data. However, they both focus largely on physical IPV; only one of the four abuse subscales measures psychological aggression. Furthermore, items on the CTS2 psychological aggression subscale primarily assess intimidation and denigration to the exclusion of other forms of emotional abuse. Having greater refinement in the assessment of non-physical forms of abuse would be especially helpful in understanding potential outcomes of partner-ideal discrepancies. Perhaps certain kinds of emotional abuse, such as denigration and hostile withdrawal, are more common control strategies than isolation or intimidation. Thus, I decided to assess physical IPV using the revised version of the CTS (CTS2; Straus, et al., 1996) and emotional abuse 
using the Multidimensional Measure of Emotional Abuse (MMEA; Murphy \& Hoover, 1999).

Physical IPV. The CTS2 consists of 78 items asking about both perpetration and victimization. Participants are asked to indicate which of several frequency ranges contains the number of times they and their partner each engaged in certain behaviors within the past six months, ranging from once to more than 20 times, with additional options for having happened before the past six months and having never happened. In addition to one subscale assessing negotiation (a nonviolent behavior; e.g., "I suggested a compromise to a disagreement"), there are four subscales relating to IPV: physical assault (e.g., "I slapped my partner”), psychological aggression (e.g., "I insulted or swore at my partner"), injury (e.g., "I had a sprain, bruise, or small cut because of a fight with my partner"), and sexual coercion (e.g., "I used threats to make my partner have sex"). An estimate of the incidence of each kind of behavior/outcome is obtained by adding together the midpoints of the chosen response options for items in a given category (e.g., a behavior reported as having happened " $3-5$ times [in the past six months]" is treated as having happened four times). Straus et al. (1996) recommends coding the response option "more than 20 times" as 25.

Past research has supported the reliability and validity of the CTS2 (Brock et al., 2015; Straus et al., 1996). Cronbach's alpha for the subscales range from .79 to 95 (Straus et al., 1996). The present study used the CTS2 subscales of physical assault and injury to assess physical IPV, which involves administering a total of 36 items. Straus et al. (1996) stated that researchers need not administer the entire scale if a study only 
focuses on certain types of IPV; the subscales of interest can be administered separately. In past research, the physical assault and injury subscales have correlated at .42 for women and .91 for men (Straus et al., 1996).

Table 15 shows descriptive statistics, internal consistencies, and subscale intercorrelations for women in the present sample. Table 16 shows the equivalent statistics for men. These tables use the sum of the coded responses, which I refer to as "raw scores" to differentiate them from scores transformed in order to more closely conform to a normal distribution (see the Results section). Subscale scores can range from zero to 300 for physical assault and from zero to 150 for the injury subscale. Similar to past research (e.g., Straus et al., 1996), Cronbach's alphas for the subscales were high. Prevalence rates, defined as the percentage of individuals who experienced one or more act in the past six months, were lower than reported by Straus et al. (1996). However, the recall period used in the present study was half that used by Straus et al. (1996; i.e., six months instead of one year). For men, chronicity rates - defined as raw injury scores among participants reporting one or more incident in the past six monthswere lower for injury and higher for physical assault than reported by Straus et al. (1996). For women, all chronicity statistics were higher than in Straus et al. (1996), some substantially so. These differences might reflect the increased reliability of reports of IPV collected through online formats (Brock et al., 2015).

Emotional abuse. Emotional abuse was assessed using the MMEA (Murphy \& Hoover, 1999). The MMEA consists of 54 items. For each item, respondents are asked to indicate how often both they and their partner each engaged in specific behaviors using 
a response scale that is nearly identical to that of the CTS2 (there is a small phrasing difference in the response option for items that occurred before but not during the time frame under consideration). The MMEA asks participants to consider acts that happened within the past six months.

The MMEA has four subscales. Dominance/intimidation (e.g., "Became angry enough to frighten the other person") measures behaviors intended to create fear or submission. Restrictive engulfment (e.g., "Tried to stop the other person from seeing certain friends or family members") includes behaviors meant to socially isolate the partner. Denigration (e.g., "Criticized the other person's appearance”) focuses on humiliating behaviors. Last, hostile withdrawal (e.g., "Acted cold or distant when angry") focuses on withdrawal behaviors presumably intended to punish the partner and foster attachment insecurity. Factor analysis has shown that these subscales measure distinct but related constructs (Murphy \& Hoover, 1999). Scores are coded in the same way as the CTS2 (i.e., the midpoint of the scale, with "more than 20 " occurrences coded as 25). Unlike the CTS2, subscale scores can also be summed to create an aggregate emotional abuse score.

The MMEA has demonstrated good reliability and validity (Murphy \& Hoover, 1999; Ro \& Lawrence, 2007; Taft, Murphy, King, Musser, \& DeDeyn, 2003). Each of the subscales positively correlate with the physical abuse perpetration subscale of the original CTS, ranging from .45 to .74 (Murphy \& Hoover, 1999). The full scale and two of the subscales, denigration and restrictive engulfment, have moderate, positive correlations with the PRQC measure of relationship satisfaction (which is also used in the 
present study; Brock et al., 2015). Internal consistency coefficients for the subscales of the MMEA have ranged from .83 to .89 (Murphy \& Hoover, 1999), with an alpha of between .77 and .94 for the total scale (Ro \& Lawrence, 2007; Taft et al., 2003).

However, one study of newlyweds found that while internal consistency was high for the overall scale, it varied widely for each of the subscales (with alphas between .55 and .74 for women's perpetration and .63 and .92 for men's perpetration) and varied between self-report and partner-report items (Ro \& Lawrence, 2007). Thus, the authors promoted using the aggregate scale. Even without examining the individual subscales separately, the MMEA represents a broader range of emotional abuse tactics than those captured within the CTS2 psychological aggression scale (which Ro and Lawrence [2007] found to have poor internal reliability, ranging from .54 to .67 ).

Descriptive statistics and internal consistency coefficients for the present sample are given in Table 17 for women and Table 18 for men. As with the tables reporting statistics on the CTS2 subscales, scores are reported in their coded, untransformed scale. Scores on the total MMEA perpetration and victimization subscales can each range from zero to 700. Cronbach's alphas were high for all aggregate and subscale perpetration and victimization scores in both women and men. Prevalence rates were much higher for MMEA scores than on the CTS2 subscales, which aligns with past research (Ro \& Lawrence, 2007). Further, average MMEA scores were higher than reported by Ro and Lawrence (2007) for both women and men. In fact, over three-quarters of both female and male participants reported perpetrating and experiencing, respectively, at least one emotionally abusive behavior in the past six months. 
Demographics and relationship/household characteristics. Participants also provided demographic information (i.e., age, race, sexual orientation, education, employment status, and annual income; questions are listed in Appendix G) and features of their current and prior romantic relationships (i.e., relationship legal status, length, cohabitation, number of past relationships, number of children, and number of children living in the household; Appendix H). Many of these characteristics have been linked to relationship satisfaction and/or IPV in past research.

Data quality questions. At the end of the questionnaire, participants were asked three questions assessing data quality (Appendix I). The first question asks about the privacy of the setting in which participants completed the survey. The second asks about technical issues. The third asks participants to subjectively evaluate the quality of their own data (i.e., whether they engaged in rushed or inattentive responding). Participants are assured that their compensation will not be affected by their response.

\section{Procedure}

All study materials and protocols were approved by the university Human Subjects Research Review Committee. Participants were recruited for an online study of relationships through Amazon's Mechanical Turk (MTurk) during October and November of 2017. First, interested individuals completed a brief screening questionnaire to determine eligibility for study participation (see Appendix J). Eligible participants then indicated their consent to complete the study (see Appendix K). After answering a few questions about their current and previous romantic relationships, they completed the measures of partner-gender ideology, partner-ideals, partner-perceptions, 
relationship satisfaction, IPV, and demographics. The partner-ideals and partnerperceptions measures were presented in a counterbalanced order across participants. The questionnaire also included additional instruments (i.e., the measures of partner-ideals and partner-perceptions created by Fletcher et al. [1999], a measure of satisfaction with power in the relationship, and a 3-item measure of partner responsiveness) that are not examined in this dissertation but that will allow for the examination of competing hypotheses in future manuscripts. At the end of the survey, participants answered a few questions assessing the quality of their data, were given the option to email their partner an invitation to participate in the study, and received contact information for the researchers and a domestic violence hotline. Participants received $\$ 1.50$ through MTurk for completing the questionnaire.

Individuals completing the partner survey (i.e., those recruited through an MTurk Worker) completed a survey that was nearly identical to the MTurk survey. However, individuals taking the partner survey were presented with the consent form and asked for their partner's Worker ID (Appendix L) before completing the eligibility questionnaire. Participants who completed the partner survey were asked to provide an email address (Appendix M) and were emailed a $\$ 1.50$ gift card to Amazon.com.

\section{Data preparation and screening}

Dyad matching. A total of $n=313$ participants recruited through MTurk (37.8\%) agreed to send their partner a link to the partner survey. Among participants who did not agree to send the survey, common reasons included their partner's anticipated lack of time, interest, or computer savvy. Some participants were concerned 
about how their partner would react to the survey content (e.g., "he could look at some major critical points at the survey and evaluate our relationship then make a choice or something," "I really don't need another discussion/argument on our messed up marriage and I'm sure it will start one"). Eight participants declined to send the survey themselves but provided their partner's email address; I did not contact these partners because such direct contact was not in the IRB-approved study protocol. Ultimately, eighty-one individuals $\left(n_{\text {women }}=42 ; n_{\text {men }}=39\right)$ completed the partner survey. Data were matched within couples using the first participant's MTurk Worker ID. Two women and one man completing the partner survey provided partner Worker IDs that did not match any collected through MTurk.

The present study only analyzed data from participants recruited through MTurk, including those whose partners did not participate. As previously discussed, it was important to the present study to prevent the potential context effects of being recruited through one's partner from impacting results. There are tentative plans to analyze the dyadic data at a later point in time.

Data screening. First, data were screened for quality. Because of an issue with Qualtrics quota settings (i.e., it is likely that multiple people started the survey simultaneously and bypassed the increment settings), $n=371$ women and $n=457$ men completed the questionnaire. Sixteen participants $\left(n_{\text {women }}=7, n_{\text {men }}=9\right)$ were excluded from analyses because they voluntarily indicated that they provided poor-quality data. Participants who failed the instructed response item embedded in the partner-ideals scale 
$\left(n_{\text {women }}=20, n_{\text {men }}=42\right)$ were also removed, as recommended by Meade and Craig (2012). No participants were excluded for a lack of variation in their responses across scales.

Participants were flagged if they complete the survey unusually quickly or if they contain outliers on the measures. Data in these cases were manually screened for problematic response patterns that indicate bogus or inattentive responding (such as a lack of variation in response choices). No such cases were identified. Cases were not removed just for containing outliers in the absence of other suspicious attributes.

The distributions of item values were examined to ensure that all values were within the expected range, and no problems were discovered. Skew and kurtosis were also examined at the item level. All items for the gender ideology, gendered partnerideals, and gendered partner-perceptions had acceptable skew and kurtosis. Items in the MMEA scale had relatively high skew and kurtosis (i.e., approaching the cutoffs recommended by Kline [2011]). CTS2 items tended to have very high skew and kurtosis, as was expected based on previous reports. Skew and kurtosis for the aggregate scales are discussed below in the section on checking statistical analyses.

Next, missingness was examined. All women in the final sample completed the entire MRNI-SF, masculine ideals, and masculine perceptions scales. There was a tiny amount of missingness in the equivalent measures completed by men; the FIS, feminine ideals, and feminine perceptions scales were each missing fewer than two data points across all male participants. One man left one item from each of the CTS2 physical and injury victimization subscales blank, resulting in a $0.02 \%$ missing data rate on each scale. There was some missingness in the MMEA; however, missingness appeared generally 
randomly distributed across items and individuals. Six individuals $\left(n_{\text {women }}=3, n_{\text {men }}=3\right)$ left either one or two MMEA perpetration items blank, resulting in a missing data rate of $0.02 \%$ for women and $0.04 \%$ for men. Nine individuals $\left(n_{\text {women }}=6, n_{\text {men }}=3\right)$ left at least one MMEA victimization item blank: seven left one item blank, one left two items blank, and one man left eight items blank. Because this last participant was missing data on $28.6 \%$ of the MMEA victimization scale, his responses on this scale were not used (which slightly reduced the sample size for Hypotheses $3 b$ and $3 c$ ). Excluding this participant, missing data rates on the MMEA victimization scale were $0.07 \%$ for women and $0.04 \%$ for men. Missing values on the CTS2 and MMEA were replaced with the mean value of the corresponding victimization or perpetration scale.

I was curious whether scores on the IPV scales varied as a function of the setting in which participants completed the survey (i.e., alone, with a partner nearby who couldn't see the responses, or with a partner nearby who could see the responses). I was primarily concerned that having a partner nearby could reduce reports of victimization or perpetration out of self-presentation concerns or fear of reprisal. The sample sizes of the groups were too unbalanced to make a truly meaningful comparison of IPV scores; only $1.5 \%$ of women's and $1.0 \%$ of men's partners were nearby and could see their responses while completing the survey. Regardless, a series of Kruskal-Wallis tests (used because raw IPV scores are ordinal) did not reveal any significant differences in men's or women's reports of IPV victimization or perpetration based on reported survey setting.

\section{Results}




\section{Preliminary analyses: Measures of feminine and masculine partner-ideals and - perceptions.}

The results presented in Chapter 3 gave preliminary support for the hypothesized factor structure of the masculine partner-ideals and-perceptions measures. However, there were several problems with feminine partner-ideals and -perceptions measures; overall model fit was inadequate, and there were several indicators with low and/or nonsignificant factor loadings. To avoid drawing conclusions from potentially faulty measures, confirmatory factor analyses were conducted on participants in the final sample who provided complete or nearly-complete data on the measures.

CFA of feminine-ideals and-perceptions measures. The sample consisted of $n=$ 406 men, which should provide sufficient statistical power based on the recommendations of Bentler \& Chou (1987) and MacCallum et al. (1996). A detailed explanation of these guidelines was given in the Method section of Chapter 3. Before conducting analyses, bivariate correlations were examined to identify potential collinearity problems. No feminine-ideal items correlated above $r=.71$. Most of the highest correlations were between items on the purity subscale, and it is expected that items on the same subscale should correlate more highly with each other than with items on other subscales. Bivariate correlations on the feminine-perceptions measures were all below $r=.60$.

Five-factor confirmatory models of the feminine partner-ideals and -perceptions measures were tested using maximum likelihood estimation in AMOS version 25 structural equation modeling software. The models tested were the same as those 
described in Chapter 3: each consisted of an oblique, five-factor model with between nine and eleven indicators loading on each factor. The variance of each factor was set to one.

First, standardized loadings were examined for low values. Compared to the pilot study described in Chapter 3, there were fewer obvious problems with individual items. All item loadings were significant at $p<.001$, and the lowest standardized loading was .31. Factor loadings for the feminine-ideals measure are provided in Table 19. While the emotionality factor still had the two lowest standardized loadings, all were higher than observed in Chapter 3. This pattern might be explained by better data screening; less attentive responding on the pilot study could have artificially decreased loadings.

As with the feminine-ideals measure, there were fewer item-level problems than identified in Chapter 3. However, several problems remained. While all standardized loadings were positive, they were as low as .01 . Table 19 also shows the factor loadings for the feminine-perceptions measure. As in the pilot study, both the stereotypic images/activities and emotionality factors had a higher proportion of low and very low loadings compared to the other factors. Interestingly, the specific stereotypic images/activities items with lower loadings pertained to physical attributes and styling rather than activities, which was found in the pilot study. It is unknown why these items had low loadings. There appeared to be no other clear patterns to the other items with lower loadings.

Second, latent factor correlations were examined. All subscales on both the feminine-ideals and -perceptions measures were significantly correlated, ranging 
from .19 to .68 for feminine-perceptions and from .24 to .78 for feminine-ideals. The smallest of these were for feminine-perceptions: caretaking with dependency/deference and caretaking with emotionality both correlated at $r=.19$. Table 20 shows correlations between the latent factors.

Third, overall model fit was assessed. The chi-square value was significant for both the feminine partner-ideals measure, $\chi^{2}(935)=3001.93, p<.001$, and the feminine partner-perceptions measure, $\chi^{2}(935)=3046.01, p<.001$, suggesting poor model fit. Alternative fit indices also suggested that model fit was poor for both measures, $\mathrm{CFI}_{\text {ideals }}$ $=.75, \mathrm{RMSEA}_{\text {ideals }}=.07 ; \mathrm{CFI}_{\text {perceptions }}=.59, \mathrm{RMSEA}_{\text {perceptions }}=.08$. Given the poor model fit, it is important not to over-interpret problems identified with individual items and factor correlations; Brown (2006) notes that misspecified models produce biased parameter estimates.

In Chapter 3, I noted that the poor model fit of the feminine-ideals and perceptions measures could be a result of model complexity rather than a true issue with model validity. This possibility can be examined indirectly by comparing the fit statistics of the created measures to those of the FIS, which has the same number of items and the same hypothesized factor structure. Readers will recall that a CFA of the FIS has never been published, so there was no external benchmark against which to compare the pilot study CFA results. A detailed discussion of the FIS CFA with the data in the present study is beyond the scope of this study. However, model fit of the FIS with data collected in the present study was better than that of the created measures, $\chi^{2}(935)=$ 2684.77, $p<.001, \mathrm{CFI}=.87$, RMSEA $=.07$. Nevertheless, fit indices for the FIS do not 
meet the commonly-accepted guidelines suggested by Hu and Bentler [1999]. These results perhaps support Marsh et al.'s [2004] argument that these guidelines should be applied thoughtfully rather than strictly, particularly for highly complex models. Additionally, all indicators loaded significantly on their respective factors, with all standardized loadings at or above .55 . Thus, it seems unlikely that the problems with the created feminine-ideals and-perceptions measures can be dismissed as merely the result of high model complexity. Unfortunately, it is unclear where the problem lies in the created measures.

It is possible that the problem with the overall model fit statistics lies not with the items themselves, but rather with the hypothesized model structure. Thus, plausible alternative models were also considered: 1) a single-factor, rather than five-factor, model (i.e., all indicators load on exactly one factor), 2) a five-factor model with a second-order general feminine-ideals/perceptions factor (i.e., instead of correlating with each other, all seven factors load onto the higher-order latent factor), and 3) a six-factor model where one factor is a general feminine-ideals/perceptions factor that is orthogonal with the other five specific factors. All three of these alternative models can be thought of as explicitly including a broad feminine-ideology/perceptions factor. All models retain the hypothesized relationship between the items in each subscale and the associated firstorder latent factor. The last alternative model conceptualizes each feminine-ideal as being caused by both feminine-ideals in general and feminine-ideals in regards to a specific domain. With the exception of the six-factor model (which is more complex 
than the hypothesized model), each alternative model is next compared to the less parsimonious hypothesized model.

The fit of nested models can be compared using the chi-square difference test (Kline, 2011). Using this strategy, more complex models are preferred unless their fit is significantly worse than the simpler model. Because the chi-square difference test can be overly sensitive with large sample sizes (Chen, 2007), Cohen's (1992) $w$ was used to estimate the effect size of the chi square difference test value. Alternative fit indices were also compared.

All chi square difference tests were significant. Similar patterns of results were observed for both the feminine-ideals and -perceptions measures. Cohen (1992) proposed that appropriate effect size cutoffs for $w$ were .10 for a small effect size, .30 for medium, and .50 for large. Using these conventions and the alternative fit indices, the single-factor model fit moderately worse than the hypothesized model, whereas adding a second-order factor to the hypothesized model fit slightly worse. The six-factor model fit slightly-to-moderately better than the hypothesized one. Table 21 compares chi square values and alternative fit indices of each model considered as well as results of the chi square difference test.

However, even the six-factor feminine models had less than desirable fit, based on the chi square tests and CFIs (although the CFI for feminine-ideals was approaching a lenient definition of acceptability at .87). Additionally, the item-level results for this model were less interpretable than for the hypothesized model. Ten feminine-ideals items had nonsignificant loadings at the $p<.05$ level, and nearly a third of the 
standardized loadings on the general ideals factor were very low or negative. There was an even greater number of item-level problems with the six-factor feminine-perceptions measure, as would be expected given the poorer fit statistics.

To summarize, neither the hypothesized feminine-ideals and -perceptions factor models nor the plausible alternative models yielded both acceptable model fit statistics and clearly interpretable item-level parameter estimates. Unfortunately, it is beyond the scope of this dissertation to engage in exploratory analyses or extensive measurement modifications in further attempts to create a measure with acceptable fit statistics and good parameter estimates. For now it must suffice to say that more work is needed on the measurement of feminine partner-ideals and -perceptions.

As determined before analyses began, hypotheses utilizing the women's measures would only be tested if evidence supported the measures' reliability. Unfortunately, results showed that the measures were problematic. Thus, Hypotheses 1a, 1b, 3c, 3d, were not tested. Additionally, H2a and H2b were only tested using women's data (i.e., the masculine-relevant measures).

CFA of masculine-ideals and-perceptions measures. Confirmatory factor analyses were conducted on both the masculine-ideals and the masculine-perceptions measures to verify their factor structures. The sample consisted of $n=344$ women, which should provide sufficient statistical power (Bentler \& Chou, 1987; MacCallum et al., 1996). Before conducting analyses, bivariate correlations were examined to identify potential collinearity problems. The highest correlations were $r=.91$ for masculine-ideal 
items and .92 for masculine-perceptions. However, higher correlations were between items on the same hypothesized factor and were thus not necessarily cause for concern.

Seven-factor confirmatory models of the masculine partner-ideals and perceptions measures were tested using maximum likelihood estimation in AMOS version 25 structural equation modeling software. The models tested were the same as those described in Chapter 3: each consisted of an oblique, seven-factor model with exactly three indicators loading on each factor. The variance of each factor was set to one.

All factor loadings were significant for both measures. Standardized item loadings were at least .50 for ideals and .47 for perceptions, which are acceptable. These results provide evidence that the items are acceptable indicators of the respective latent factors they were intended to measure. Factor loadings for each masculine measure are provided in Table 22.

Factor correlations for the masculine-ideals measure were similar to those found in the pilot study, although some were slightly higher (see Table 23). This pattern could be explained by better data screening; inattentive responding on the pilot study could have decreased correlations. All correlations were significant except for that of selfreliance through mechanical skills and restrictive emotionality, and most were of moderate to large magnitude. Similarly, most of the factor correlations on the masculineperceptions measure were higher than those found in the pilot study. All were significant, and most were of small-to-moderate magnitude. 
Next, overall model fit to the data was assessed. The chi square value was significant for both the masculine partner-ideals measure, $\chi^{2}(168)=419.05, p<.001$, and the masculine partner-perceptions measure, $\chi^{2}(168)=393.08, p<.001$, suggesting poor model fit. However, alternative fit indices suggested that model fit was acceptable to good for both measures, $\mathrm{CFI}_{\text {ideals }}=.94, \mathrm{RMSEA}_{\text {ideals }}=.07 ; \mathrm{CFI}_{\text {perceptions }}=.94$, $\mathrm{RMSEA}_{\text {perceptions }}=.06$.

While the fit of the hypothesized models were acceptable, theoretically reasonable alternative models were also considered on principle. Four plausible alternatives were considered: 1) a single-factor model (i.e., all indicators load only on one latent factor), 2) a seven-factor model with a second-order general masculine-ideals/perceptions factor (i.e., instead of correlating with each other, all seven factors load onto one higher-order latent factor), and 3) an eight-factor model where one factor is a general masculineideals/perceptions factor that is orthogonal with the other seven factors. These were similar to the alternative models examined for the feminine measures, except they included the seven masculine dimensions instead of the five hypothesized feminine dimensions. These models were also similar to the alternatives to the MRNI-SF considered by Levant et al. $(2013,2015)$, with the exception of the single-factor model. The single-factor model was primarily included to determine whether separating the items into the seven hypothesized factors offered any benefit over having one large, aggregate measure of masculine-ideals and-perceptions. Thus, this comparison was intended to establish the utility of the factors. 
The second and third alternative models are predicated on the possibility that there is a general factor relating to each specific component of masculine-ideals and perceptions, respectively. The distinction between these two models is in whether those specific factors are subsumed by the general factor or simply correlated with it. Levant et al. (2013) preferred the eight-factor model for the MRNI-SF, which they referred to as a "bifactor" model because it splits latent factors into general and specific components. In contrast, Levant et al. (2015) found that the seven-factor model of the MRNI-SF fit better than the eight-factor model, although a modified eight-factor model that removed three of the 21 correlations between the seven factors fit marginally better than any of the alternatives. This small variability in model fit even within the same measure demonstrates how important it will be to attempt to replicate the results of the masculinemeasures found in this study.

The fit of the nested models for the masculine-measures were compared using the chi square difference test (Kline, 2011), Cohen's (1992) $w$ as a measure of effect size, and alternative fit indices. All chi square difference tests were significant. Similar patterns of results were observed for both the masculine-ideals and -perceptions measures. Based off the conventions proposed by Cohen (1992) for $w$ and the alternative fit indices, the single-factor model fit substantially worse than the hypothesized model. Adding a second-order factor to the hypothesized model fit slightly worse. The eightfactor model fit slightly better than the hypothesized one. Results of the model comparisons are in Table 24. 
While the eight-factor model had slightly better fit statistics than the hypothesized model, inspection of the parameter estimates revealed some problems. Interestingly, problems were different for the ideals and perceptions measures. For the masculineperceptions measure, adding the orthogonal general masculine ideals factor caused item loadings on five out of seven factors to become negative (i.e., avoidance of femininity, negativity toward sexual minorities, self-reliance through mechanical skills, dominance, and importance of sex). This is conceptually problematic, given that all items should positively reflect ideals regarding traditional masculinity. However, most standardized loadings were of an acceptable magnitude (save two items on the toughness factor), and all items loaded positively onto the general factor. Adding the general factor also caused the seven latent factors to have very low correlations or even negative correlations (many of which were also nonsignificant).

On the eight-factor masculine-perceptions measure, most item loadings were of acceptable magnitudes on the seven factors. However, loadings were negative for the toughness factor. Again, it is puzzling that factors have different signs when they should all be positively related. Additionally, most loadings on the general masculine perceptions factor were low or very low, and one was negative. Latent factor correlations showed the same problems as in the masculine-ideals measure. The problems with parameter estimates on the eight-factor model, combined with only a slight improvement in fit statistics, supports retaining the original hypothesized model for analyses. Thus, the hypothesized seven-factor models of masculine-ideals and -perceptions were used in analyses. 
Descriptive and comparative data. In addition to exploring the factor model of the masculine measures, it is also informative to examine descriptive results and mean comparisons of the scale scores. Examination of the aggregate and subscale means reveals differences in masculine-ideals versus masculine-perceptions (see Table 13). Most individual dimensions of masculine-ideals were considered at least somewhat desirable, with self-reliance through mechanical skills the most desirable. However, negativity toward sexual minorities and restrictive emotionality were somewhat undesirable.

Patterns of mean masculine-perceptions differed somewhat (see Table 13). Women perceived their male partners as conforming, on average, least to the norm of negativity toward sexual minorities and most to the norm of avoidance of femininity. Paired $t$-tests revealed significant differences between masculine-ideals and -perceptions for the aggregate scale, $t(343)=-4.76, p<.001, d=-.25$, such that men were generally perceived as conforming more than desired to male role norms. This pattern was also found for the specific role norms of avoidance of femininity, $t(343)=-11.55, p<.001, d$ $=-.63$, negativity toward sexual minorities, $t(343)=-7.75, p<.001, d=-.41$, importance of sex, $t(343)=-2.51, p=.01, d=-.14$, and restrictive emotionality, $t(343)=-13.65, p$ $<.001, d=-.75$. In contrast, male partners were perceived as conforming less than desired to the norms of self-reliance through mechanical skills, $t(343)=8.35, p<.001, d$ $=.47$, and dominance, $t(343)=7.57, p<.001, d=.41$. The difference between ideals and perceptions regarding toughness was not significant, $t(343)=.69, p=.49, d=.04$. These results suggest that 1) individual masculine-ideals vary in the extent to which they are 
desirable, 2) men conform more than desired to some masculine-ideals and less than desired to others, and 3) the magnitude of discrepancies between women's masculine partner-ideals and -perceptions ranges from statistically insignificant to large, depending on the specific dimension.

\section{Checking statistical assumptions for hypothesis tests.}

Next, data on all measures were checked for the fulfillment of statistical assumptions for each hypothesis test. The calculation of Pearson's $r$, used to test Hypotheses 1 and 3, assumes an absence of outliers, a linear relationship between variables, and homoscedasticity. Mediation analyses with ordinary least squares regression used to test Hypothesis 2 assume linear relationships between the variables, normally distributed errors, homoscedasticity, and independence of errors. However, the analysis used in this manuscript is robust to violations of the assumption of normally distributed errors except with very small sample sizes (Hayes, 2013). The assumptions for each hypothesis were checked using the procedures described below. Readers should recall that because the CFAs for the feminine measures were problematic and thus hypotheses using men's data could not be tested, the analyses described below relating to IPV measures only utilize women's data.

Scale and subscale means were calculated for each measure examined in this document. For the FIS, MRNI-SF, partner-ideals and partner-perceptions measures, and the PRQC, means were calculated for individuals providing data on a minimum of $85 \%$ of the scale or subscale under consideration. This is equivalent to mean substitution, which past research has found to be roughly equivalent to more complex methods of 
dealing with item-level missingness when rates of missingness are relatively low (Parent, 2012). For the CTS2 and MMEA subscales, mean substitution was used (except, as previously discussed, for the one participant who left nearly one third of the MMEA victimization items blank).

Next, distributions of scale and subscale scores were examined for outliers. There were a number of outliers in the various scales. Outliers ranged to great extremes in the IPV variables, as expected. For example, one woman's MMEA perpetration score was almost nine standard deviations above the mean at 602 . CTS2 subscale scores ranged to just above thirteen standard deviations above the mean. As discussed previously, univariate outliers were retained because they were not determined to be the result of incorrect calculation or obvious response sets (i.e., no variation in the participant's responses to items in that measure).

Skew and kurtosis of the aggregate scales were then examined. Before beginning analyses, it was decided that scales with skew larger than an absolute value of three or kurtosis larger than 10 (based on Kline [2011]) would be subjected to a nonlinear transformation to increase normality. While the distribution of PRQC scores was somewhat negatively skewed $($ skew $=-1.70$, kurtosis $=2.92)$, as expected, it was not extreme enough to warrant transformation. MRNI-SF scores were close to normal for the aggregate scale $($ skew $=.54$, kurtosis $=-.06)$, although on the subscales skew ranged from -.66 to 1.74 and kurtosis ranged from -.43 to 3.33. Masculine-ideals and -perceptions scores were both slightly negatively skewed $\left(\right.$ skew $_{\text {ideals }}=-.41$, kurtosi $_{\text {ideals }}=.35$; skew $w_{\text {perceptions }}=-.18$, kurtosis $\left._{\text {perceptions }}=-.34\right)$. Skew and kurtosis for all subscales were 
within the predetermined thresholds for acceptability. The distribution of masculine partner-ideal discrepancies was slightly positively skewed but also within the predetermined thresholds $($ skew $=.85$, kurtosis $=.31)$.

As anticipated, skew and kurtosis were high for the IPV measures. The distributions of MMEA scores were beyond the predetermined thresholds of skew and kurtosis for both perpetration $($ skew $=3.92$, kurtosis $=21.76)$ and victimization $($ skew $=$ 3.27, kurtosis $=12.96)$. CTS2 scores were even more nonnormal; this was true for both the physical assault $\left(\right.$ skew $_{\text {perp.. }}=8.14$, kurtosis $_{\text {perp. }}=72.11 ;$ skew $_{\text {victim }}=8.13$, kurtosis $_{\text {victim }}$ $=71.11)$ and injury $\left(\right.$ skew $_{\text {perp. }}=8.71$, kurtosi $_{\text {perp. }}=81.37 ;$ skew $_{\text {victim. }}=9.99$, kurtosis $_{\text {victim }}$. $=112.71)$ subscales.

To address the distributions' deviations from normality, several different nonlinear transformations were attempted: taking the square root of the total scale score, taking a base ten logarithm of the total scale score, and taking the inverse of the total scale score (Tabachnick \& Fidell, 2007). For all of these transformations, one was first added to the total scale score to avoid having undefined transformed values for individuals with total score values of zero.

For MMEA scores, a log transformation best reduced nonnormality (skew perp. $=.06$, kurtosis $_{\text {perp. }}=-1.03 ;$ skew $_{\text {victim. }}=.04$, kurtosi $\left._{\text {victim. }}=-1.13\right)$. While the inverse function was somewhat more successful at reducing kurtosis, it also resulted in a bimodal distribution. Additionally, examination of correlations between differently transformed MMEA perpetration scores and PRQC scores as well as the residuals plots regressing PRQC scores on MMEA perpetration scores (relevant to H2) showed that the log 
transformation resulted in the most linear relationship and the most evenly distributed errors in the regression. The same pattern was found when plotting MMEA victimization scores against masculine-perception scores (H3). Thus, MMEA scores were logtransformed for hypothesis testing.

The same three common transformations were also attempted for scores on the CTS2 physical assault and injury subscale. For the physical assault subscale, only the inverse transformation brought skew and kurtosis within an acceptable range $\left(\right.$ skew $_{\text {perp. }}=$ -2.24, kurtosis $_{\text {perp. }}=3.36 ;$ skew $_{\text {victim. }}=-1.96$, kurtosi $\left._{\text {victim. }}=2.11\right)$. However, histograms of inverted scores still showed substantial nonnormality, with the vast majority of scores falling at one (i.e., no IPV). Additionally, plots of the transformed scores failed to show a linear relationship with PRQC and masculine-perception scores. The distributions of the CTS2 injury subscales were even more problematic. While the inverse transformation resulted in the best skew and kurtosis of the options attempted (skew $w_{\text {perp. }}=$ -3.88, kurtosi $_{\text {perp. }}=13.84 ;$ skew $_{\text {victim. }}=-3.25$, kurtosis $\left._{\text {victim. }}=9.22\right)$, they were still beyond the acceptable threshold. A variety of additional transformations in the Box-Cox family were attempted using SPSS syntax from Osborne (2010), but none gave satisfactory results. Because none of the transformations applied to the CTS2 subscales reduced the skew and kurtosis to acceptable levels while also fulfilling assumptions of linearity on the planned hypothesis tests, scores on both subscales were dichotomized such that $0=$ no IPV and $1=$ any IPV. This approach is common in other research utilizing CTS2 data (e.g., Jaspaert \& Vervaeke, 2014). In the language of Straus et al. (1996), analyses using 
these subscales will now be exploring "prevalence" (i.e., any incidence of IPV) rather than "chronicity" (i.e., the total number of events).

Next, distributions were examined for multicollinearity by examining correlations between pairs of predictor variables. If scales in different measures have correlations higher than .90 (Tabachnick \& Fidell, 2007), analyses involving both measures cannot be run. No such extremely large correlations were found.

Multivariate outliers were identified by calculating the Mahalanobis distance. As suggested by Tabachnick and Fidell (2007), I planned to remove relevant values for cases with a Mahalanobis distance significant at $p<.001$. There were no multivariate outliers for H1c. The variables tested in H1d had six outliers; these cases were excluded for the analysis. While there were no outliers for the correlation between aggregate masculine perceptions and the total MMEA or CTS2 physical assault subscale (H3a), there were four for the CTS2 injury subscale. These values were excluded. For H3b, there were two outliers on the MMEA, one on the CTS2 physical assault subscale, and two on the CTS2 injury subscale; these values were excluded when testing relevant hypotheses.

Linearity of the association between variables was assessed by visually examining scatterplots. Scores on the plots appeared to reasonably follow a linear pattern rather than other possibilities such as a curvilinear pattern or no association. The normality of residuals for the linear (i.e., non-logistic) paths in $\mathrm{H} 2 \mathrm{a}$ and $\mathrm{H} 2 \mathrm{~b}$ was confirmed by visually examining P-P plots of the residuals. Scores tended to follow a straight line, suggesting that the residuals were acceptably linear.

\section{Hypothesis One}


Hypothesis One addresses the relationship between traditional gender ideology and gendered partner-ideals. To test the hypotheses that gender ideology correlates with both aggregate and specific dimensions of gendered partner-ideals, scores for partnergender ideology and partner-ideals were calculated at the aggregate and subscale levels. It was expected that both aggregate and specific components of gender ideology and gendered partner-ideals would be positively correlated. For example, women with traditional masculine ideologies would have stronger masculine partner-ideals than women who do not hold a traditional masculinity ideology. Similarly, endorsement of any specific element of gender ideology would positively and significantly correlate with the equivalent partner-ideal.

H1a and H1b focused on men's femininity ideology and feminine-perceptions. Unfortunately, these hypotheses could not be tested because of the problems with the feminine measures revealed in the factor analysis described earlier.

To test H1c, I calculated the bivariate correlation between women's total masculinity ideology and total masculine partner-ideals. The correlation was significant and large, $r(344)=.74, p<.001$. This means that women who generally endorse traditional masculinity ideology also hold traditional masculine-ideals, and the opposite is also true.

H1d was tested by calculating bivariate correlations between women's subscale masculinity ideology scores and subscale masculine partner-ideals scores (shown along the diagonal in Table 25). All correlations were positive and significant. The highest correlation was for negativity toward sexual minorities, $r(344)=.92, p<.001$. This was 
followed by restrictive emotionality, $r(344)=.70, p<.001$, toughness, $r(344)=.60, p$

$<.001$, avoidance of femininity, $r(344)=.59, p<.001$, self-reliance, $r(344)=.54, p$

$<.001$, importance of sex, $r(344)=.52, p<.001$, and dominance, $r(344)=.38, p<.001$.

While dominance had the smallest correlation of the seven dimensions, it was still moderate by widely accepted guidelines (Tabachnick \& Fidell, 2007). However, unexpectedly, the masculine-ideal of dominance correlated more highly with the masculinity ideology dimensions of self-reliance and toughness than with dominance. It is unclear why this would be so. Perhaps, as discussed earlier on the section on the masculine measures CFAs, adapting the items on that dimension of the MRNI-SF to apply to one's partner resulted in some construct drift.

Collectively, the results for Hypothesis 1 show that there is a positive association between women's masculine ideology and associated masculine partner-ideals. Potential interpretations of this finding are discussed in the next chapter.

\section{Hypothesis Two}

Hypothesis 2 concerns the associations among gendered partner-ideal discrepancies, relationship satisfaction, and IPV; moderated by gender. Specifically, it was hypothesized that for both men and women, gendered partner-ideal discrepancies positively predict IPV (including both physical IPV and controlling behaviors), mediated by relationship satisfaction (see Figures 1 and 2).

Because the CFAs raised doubts about the validity of the feminine measures, men's data could not be used; only women's data on the masculine measures were used to test this hypothesis. Thus, instead of including gender in a moderated mediation model, 
regular mediation was used (Model 4 of Hayes' PROCESS macro Version 2 in SPSS, 2012). The model tested became one of women's IPV perpetration predicted by masculine-ideal discrepancies and relationship satisfaction. The order of the variables in the model remained the same as in Figures 1 and 2. 10,000 bias-corrected bootstrap samples were used in the PROCESS macro to estimate confidence intervals for the indirect effect (i.e., path c') in the model. Based on Hayes' (2013) recommendations, variables were neither standardized nor centered.

It is important to note that while $\mathrm{H} 2 \mathrm{a}$ and $\mathrm{H} 2 \mathrm{~b}$ are tested using the same PROCESS model (i.e., model 4, which is a basic mediation), the parameter estimates cannot be interpreted in the same way. This is because of the differences in how the outcome variables were transformed to address the statistical assumption of normality. Readers will recall from the data screening section that MMEA scores were logtransformed whereas CTS2 subscale scores were dichotomized. The PROCESS macro automatically senses whether outcome variables are continuous or binary and estimates paths $\mathrm{b}$ and $\mathrm{c}$ using maximum likelihood logistic regression for binary outcomes (Hayes, 2013). However, total effects and estimates of effect size for indirect effects are not computed in models with a dichotomous outcome. There is no difference in the way that PROCESS calculates confidence intervals for the indirect effect.

H2a tested the mediation model with CTS2 physical assault and injury subscales as the dependent variables. Similar patterns of results were obtained for both subscales. Neither full model was significant, Nagelkerke $R_{\text {physicalassault }}^{2}=.03, p=.09$; Nagelkerke $R_{\text {injury }}^{2}=.04, p=.05$. Masculine-ideal discrepancies negatively predicted PRQC scores 
(path a), as expected, $b=-2.16, S E=.17, p<.001,95 \% \mathrm{CI}=-2.50,-1.83$. This path was the same in both models. While path b, regressing CTS2 subscales on PRQC scores, was negative as predicted, it was significant for neither physical assault, $b=-.18, S E=.14, p$ $=.18, O R=.84,95 \% \mathrm{CI}=-.45, .08$, nor injury, $b=-.27, S E=.18, p=.13, O R=.76$, $95 \% \mathrm{CI}=-.62, .08$ (see the post-hoc analyses at the end of this chapter for more on this relationship). A direct effect of masculine-ideal discrepancies on IPV (path c) is not necessary to claim mediation. Nevertheless, it was nonsignificant for both physical assault, $b=.39, S E=.58, p=.49, O R=1.48,95 \% \mathrm{CI}=-.71,1.48$, and injury, $b=.51, S E$ $=.78, p=.51, O R=1.67,95 \% \mathrm{CI}=-1.03,2.05$. Lastly, the indirect effect of masculine ideal-discrepancies was significant for neither physical assault, $b=.39, S E=.30, O R=$ $1.48,95 \% \mathrm{CI}=-.18, .98$, nor injury, $b=.59, S E=.38, O R=1.80,95 \% \mathrm{CI}=-.17,1.36$. While exact $p$ values are not computed for the indirect effects in either model, the fact that the confidence interval includes zero means that the effects are nonsignificant. Results of H2a are shown in Table 26.

To help with interpretation, Figure 3 shows the estimates of paths a, b, c, and c' (the indirect effect) for the CTS2 physical assault subscale. The equivalent parameters for the CTS2 injury subscale are shown in Figure 4. While the parameter estimates were in the anticipated direction, the data were not consistent with the mediation models proposed in $\mathrm{H} 2 \mathrm{a}$.

$\mathrm{H} 2 \mathrm{~b}$ tested the relationship between masculine ideal-discrepancies and emotional abuse perpetration, mediated by relationship satisfaction. In contrast to the models tested in $\mathrm{H} 2 \mathrm{a}$, in which paths $\mathrm{b}$ and $\mathrm{c}$ were logistic, all paths in this model were linear. The full 
model was significant, $F(1,340)=24.54, p<.001, R^{2}=.07$ (see Table 27). Path a, regressing PRQC scores on masculine ideal-discrepancies, was the same as in H2a, $b=$ 2.16, $S E=.17, p<.001,95 \% \mathrm{CI}=-2.50,-1.83$. Path $\mathrm{b}$, regressing MMEA scores on PRQC scores, was also significant and in the expected direction, $b=-.13, S E=.04, p$ $<.001,95 \% \mathrm{CI}=-.20,-.06$. The direct effect of masculine ideal-discrepancies (path c) was significant, $b=.32, S E=.14, p=.03,95 \% \mathrm{CI}=.03, .60$. The indirect effect (path c') was also significant, $b=.28, S E=.08,95 \% \mathrm{CI}=.12, .45$, providing support for the hypothesis that the relationship between women's masculine ideal-discrepancies and emotional abuse perpetration is mediated by relationship satisfaction. Practically speaking, this means that for a one-unit increase in masculine ideal-discrepancies (which is not practically meaningful for most participants, as masculine ideal-discrepancies were computed as a correlation and thus are bounded by -1 and 1), one would expect an increase in raw MMEA scores of 1.91 (reversing the log-transform by computing $10^{28}=$ 1.91) through relationship satisfaction. Additionally, the total effect was significant, $b$ $=.60, S E=.12, p<.001,95 \% \mathrm{CI}=.36, .84$. This translates to an expected increase in raw MMEA perpetration scores of $10^{.60}=3.98$ for a one-unit increase in masculine idealdiscrepancies. While statistically significant, it is important to realize that this effect is small in magnitude. Figure 5 shows the path estimates for $\mathrm{H} 2 \mathrm{~b}$.

Thus, Hypothesis 2 was partially supported. Results showed that there was an association between women's masculine ideal-discrepancies and emotional IPV perpetration, mediated by relationship satisfaction. However, this same pattern was not found for perpetration of physical assault or injury. 


\section{Hypothesis Three}

Hypothesis Three concerns the relationship between perceived partner gender role conformity and IPV. As stated earlier, men's data could not be used to test H3a because of problems with the feminine measures. MMEA scores were log-transformed to better fulfill assumptions of normality made by the statistical models tested. Because transformations applied to the CTS2 subscales failed to approximate normality, scores were dichotomized. While calculating correlations with one continuous and one dichotomous variable (i.e., point-biserial correlations) appears to be uncommon in published research, it has the advantage of indicating the strength and directionality of the association while producing the same $p$ value as would be found by comparing the groups using an independent samples $t$-test.

H3a was tested by calculating the correlation between women's masculine partner-perceptions and their reports of both physical IPV and emotional abuse victimization. There was partial support for $\mathrm{H} 3 \mathrm{~b}$; women's MMEA victimization scores positively correlated with total masculine-perceptions, $r_{p b}(344)=.15, p=.01$. The correlation between total masculine-perceptions and the CTS2 physical assault subscale was not significant, $r_{p b}(344)=.05, p=.36$. Nor was the correlation with the CTS2 injury subscale, $r_{p b}(340)=.08, p=.13$. Thus, general masculine-perceptions were only associated with MMEA victimization (and not with physical assault or injury victimization), and this association was weak. However, as shown in Table 28, achieved statistical power was low for the correlations of the CTS2 subscales with total masculineperceptions. 
To test $\mathrm{H} 3 \mathrm{~b}$, correlations were calculated between each masculine partnerperceptions subscale and women's reports of physical and emotional victimization. Among the three measures of IPV, correlations were strongest for the MMEA. Nevertheless, only two dimensions of masculine-perceptions significantly predicted MMEA scores: negativity toward sexual minorities, $r_{p b}(342)=.20, p<.001$, and restrictive emotionality, $r_{p b}(342)=.40, p<.001$. These same dimensions also predicted CTS2 injury prevalence, with a stronger association for negativity toward sexual minorities, $r_{p b}(342)=.17, p=.01$, than for restrictive emotionality, $r_{p b}(342)=.11, p=.04$. CTS2 physical assault prevalence was only significantly predicted by the dimension of restrictive emotionality, $r_{p b}(343)=.12, p=.03$. None of the other correlations were significant, and some trended in the opposite direction as anticipated. As shown in Table 28 , achieved statistical power was inadequate for the correlations with very small magnitudes. Overall, there was limited support for $\mathrm{H} 3 \mathrm{~b}$.

Thus, women's total masculine-perceptions of their partners had a small and inconsistent association with women's emotional abuse victimization. Restrictive emotionality positively predicted all three types of IPV, although the magnitude of the association with physical assault and injury was small. Lastly, negativity toward sexual minorities was associated with both injury and emotional abuse. On the whole, there were fewer and weaker associations between masculine-perceptions and IPV than would be expected based on prior research.

Some hypotheses in this chapter were supported, some were refuted, and some were inconsistently supported. The results of Hypothesis One showed that femininity 
ideology predicts feminine-ideals. The results of Hypothesis Two revealed that gender ideal-discrepancies predict emotional IPV perpetration, but not physical IPV perpetration, mediated by relationship satisfaction. Hypothesis Three found limited support for the concept that masculine-perceptions positively predict IPV. However, it is important to remember that hypothesis tests utilizing the measures of feminine-ideals and-perceptions could not be conducted because of measurement issues, so results are only based on the data provided by female-identified participants. The results of the hypothesis tests in this chapter have been summarized in Table 30 .

\section{Post Hoc Analyses}

Masculine-perceptions and relationship satisfaction. In Chapter One, it was noted that previous research on gender role conformity and relationship satisfaction tends to utilize the trait perspective rather than exploring gender roles as multidimensional. Only a handful of studies have examined perceptions of men's masculine role conformity and women's relationship satisfaction. To expand the literature on masculine-perceptions and relationship satisfaction, I calculated the bivariate correlations between masculineperceptions and women's PRQC scores. While the correlation between aggregate masculine partner-perceptions and PRQC scores was positive, $r(344)=.19, p<.001$, the direction of the relationship varied across individual dimensions. Several dimensions were positively related to relationship satisfaction, including toughness, $r(344)=.34, p$ $<.001$, dominance, $r(344)=.30, p<.001$, importance of sex, $r(344)=.27, p<.001$, and self-reliance, $r(344)=.27, p<.001$. However, restrictive emotionality was negatively related, $r(344)=-.26, p<.001$. Unrelated to relationship satisfaction were 
avoidance of femininity, $r(344)=.06, p=.25$, and negativity toward sexual minorities, $r(344)=-.08, p=.05$. These results are explored in the next chapter.

IPV and relationship satisfaction. Conducting the Hypothesis Two mediation analysis, I was surprised to discover that while relationship satisfaction significantly predicted emotional abuse perpetration (albeit with a relatively small magnitude), it did not predict physical assault or injury perpetration. This stands in contrast to the prior research reviewed in Chapter One. Readers will recall that past studies have found relationship satisfaction to predict both IPV perpetration and victimization. To explore the results of Hypothesis Two more deeply, I first calculated the correlations between PRQC mean scores and IPV perpetration and victimization on all three measures (i.e., CTS2 physical assault, CTS2 injury, and the MMEA; see Table 29). All correlations were significant and ranged from $r=-.12$ to -.42 . This finding suggests that, in spite of pre-analysis statistical screening, the failure to find path $b$ in Hypothesis Two significant could be a result of multicollinearity between PRQC scores and masculine-ideal discrepancies. In other words, perhaps neither path $\mathrm{b}$ nor path $\mathrm{c}$ in $\mathrm{H} 2 \mathrm{a}$ was significant because PRQC scores and masculine-ideal discrepancies predict similar variance in CTS2 perpetration scores. To probe this possibility, I calculated bivariate correlations between PRQC scores, masculine-ideal discrepancies, and IPV (see Table 29). Lending some support to my suspicions, PRQC scores correlated with masculine-ideal discrepancies at $r(342)=-.56, p<.001$. Like PRQC scores, masculine-ideal discrepancies were significantly correlated with all measures of IPV, with the exception of physical assault perpetration. Otherwise, the magnitude of the correlations between IPV and masculine- 
ideal discrepancies were comparable to, though slightly smaller than, those of IPV and PRQC scores. These post hoc analyses are discussed in more depth in Chapter Five. 


\section{Chapter 5. Discussion, Implications, and Limitations}

\section{Implications for Theory and Research}

The main study described in this manuscript improves upon previous research on partner-ideals by measuring gendered partner-ideals and exploring their associations with gender ideology and relationship outcomes (although hypothesis testing was limited to the masculine-ideals and-perceptions measures). This dissertation contributes to the literature by creating and factor analyzing measures of masculine-ideals and -perceptions that can be utilized in future relationship research. Additionally, it is one of few existing studies to examine the behavioral implications of conformity to specific gender role norms for IPV perpetration.

This study has implications for theory in the areas of partner-ideals, partner-ideal discrepancies, and IPV. Results show that women's endorsement of specific masculine partner-ideals mirrors their endorsement of specific dimensions of masculinity ideology, which is consistent with masculine-ideals being acquired through socialization rather than evolutionary pressures. Results also support the application of discrepancy theory to relationship research by showing that masculine-ideal discrepancies are negatively associated with relationship satisfaction. Further, there is evidence that some women use emotional abuse as a control tactic in response to masculine partner-ideal discrepancies. Unfortunately, the measurement limitations described in Chapter 4 precluded testing the same associations between partner-ideal discrepancies, relationship satisfaction, and IPV perpetration in male respondents. Correlations between men's perceived conformity to 
male roles and IPV perpetration differed from past research, suggesting that this theorized link needs further clarification.

Measure development. This manuscript describes the creation and utilization of new measures of gendered partner-ideals and partner-perceptions. As previously noted, there are currently no existing partner-report measures of gender role conformity with known measurement properties; existing measures are self-report. The measures created in this study will enable researchers to conduct work on gendered partner-ideals and gendered partner-perceptions with greater confidence.

Obviously, the measures created for this study require further evidence to support their validity. While Chapter Four described the evidence of validity for the measures of masculine partner-ideals and-perceptions, more work is needed, especially in establishing its predictive, concurrent, and discriminant validity. Hypothesis One could be interpreted as providing some support for the masculine-ideal measure's concurrent and discriminant validity (i.e., masculine-ideals should be related to but distinct from masculinity ideology). However, there is much more work to be done.

As discussed in Chapter Four, factor analysis supported the hypothesized factor structure of the masculine measures but revealed several problems with the feminine measures. This measurement limitation meant that I could only test the mediation model of IPV in the sample of women. As discussed in Chapter 1, the bulk of psychological research on IPV is focused on IPV that is both physical and perpetrated by men. Reflecting this trend, past work examining the interactive effects of partners' gender ideology on IPV focuses on men's physical IPV perpetration. I had hoped to increase 
both the depth and breadth of this line of inquiry by examining gendered partner-ideal discrepancies rather than gender ideology, emotional in addition to physical IPV, and the perpetration of both men and women. Ultimately, the project was unable to achieve this goal. However, the data on women's physical, injury, and emotional abuse perpetration have value in their uniqueness. While not the focus of this study, the descriptive statistics of IPV perpetration and victimization suggest that there could be some differences in the types of abuse perpetrated and experienced by men and women. Once adequate measures of feminine-ideals and -perceptions are available, it would be informative to retest the original moderated mediation model proposed in Hypothesis Two.

The measures of feminine partner-ideals and-perceptions would require extensive revision and validation before being utilized in future research. I would recommend starting with a critical comparison of the domains contained in the Femininity Ideology Scale with those of the Conformity to Feminine Norms Inventory. There is much less conceptual overlap between these two measures than there is between the equivalent male measures (i.e., the Male Role Norms Inventory and the Conformity to Masculine Norms Inventory). This suggests that feminine role norms could be less established in United States culture in general (varying depending on age, region, subculture, etc.) or vary depending on the context. In Chapter One, I briefly discussed some research demonstrating that men hold different ideals for women in a romantic versus nonromantic setting (Delacollette et al., 2013, Smiler \& Kubotera, 2010). Thus, perhaps the unique context of the romantic relationship shapes men's feminine partnerideals in a different way than their femininity ideology. Depending on the magnitude of 
this divergence, it might be necessary to create a measure of feminine partner-ideals using an inductive method rather than a deductive approach such as adapting an existing measure of gender ideology. Lee et al. (2010) takes such an approach in their qualitative study, as previously discussed. While the method utilized by Lee et al. (2010) has some drawbacks, some qualitative methods such as interviews could effectively overcome them by encouraging participants not only to limit their responses to the romantic context, but also to consider ideals that are so commonly accepted that they might go unmentioned in a brief survey.

With further support—and revision of the feminine measures - the measures created in this study could be used to confirm previous research on gender role conformity in the context of romantic relationships. Some past research has adapted selfreport versions of gender role conformity measures to partner-report formats (e.g., Burn \& Ward, 2005; Rochlen \& Mahalik, 2004). I have discussed the issues with these adaptations in Chapter Two of this document. With the greater focus on observable characteristics rather than internal working states or hypothetical behaviors, the measures created for the present study could offer more valid assessments of partners' actual gender role conformity.

Partner-ideals. Building upon previous work on masculinity ideology, this study identified the theoretical components of masculine partner-ideals. Confirmatory factor analysis supported the reliability of seven masculine partner-ideals matching those previously found to comprise masculinity ideology: avoidance of femininity, negativity toward sexual minorities, self-reliance through mechanical skills, toughness, dominance, 
importance of sex, and restrictive emotionality. Additionally, results indicated the importance of examining masculine partner-ideals and-perceptions as multidimensional. With the exception of negativity toward sexual minorities and restrictive emotionality, women rated most individual masculine-ideals as somewhat to very desirable. This variation between specific domains suggests that Mahalik et al.'s (2005) admonition to consider gender roles as multidimensional should be extended to the study of gendered partner-ideals.

Past research has found empirical support for the existence and importance of the stereotypically gendered domains of attractiveness/vitality—especially in men's female partners—and status/resources—especially in women's male partners (e.g., Fletcher \& Simpson, 2000). However, other research has found different stereotypically-gendered characteristics to be equally, if not more, important (e.g., Buss, Shackelford, Kirkpatrick, \& Larsen, 2001; Lee et al., 2010). Such characteristics include having a desire for a home and children, having a pleasing disposition, and ambition/industriousness. In the present study, the closest corollary to status/resources examined was dominance, and this dimension was desired less than self-reliance through mechanical skills. Avoidance of femininity, which offers no clear evolutionary benefit whatsoever, was the third most highly desired masculine partner-ideal. Complicating the issue is the fact that most studies measuring gendered partner-ideals either draw upon ideals from theory on mate selection or take an entirely bottom-up approach by asking participants to spontaneously nominate ideals. The former approach offers the advantage of being applicable to partners of either gender. However, it can also represent a lack of specificity in ideals. 
Vague items can have different connotations for each gender, such as "pleasing disposition" and "good health" as measured in Buss et al. (2001). Ignoring these potential differences in interpretation undermines measurement validity. The latter approach, letting participants freely nominate ideals, can be vulnerable to participants' lack of insight or imagination. Certain relationship or partner expectations might be so normalized that participants overlook them or are hesitant to articulate them, such as sexual fidelity. This study took a new approach by using a theory-driven measure of partner-ideals that is conceived not from an evolutionary perspective, but rather from a social roles perspective. This shifts the focus of measurement from characteristics that should vary widely between genders to those roles that are uniquely expected of each gender. The study is the first to systematically measure the degree to which women place importance on their male romantic partners' conformity to specific masculine role norms.

This study also considered a potential source of masculine partner-ideals, namely socialization. Hypothesis One was supported among women, meaning that women's masculinity ideology was positively correlated with their masculine partner-ideals. In other words, women who placed more importance on men's fulfillment of masculine role norms were more likely to desire those same attributes in a male partner. These results are consistent with gendered partner-ideals being influenced by socialization. At the same time, they raise doubt about other researchers' assumption that evolutionary pressures determine stereotypically gendered partner-ideals (e.g., Fletcher, Simpson, Thomas, \& Giles, 1999). Namely, being born a particular sex is not sufficient to establish "evolutionarily advantageous" partner-ideals; rather, gender differences in 
partner-ideals are more likely a reflection of endorsement of broader cultural ideologies. There is no obvious evolutionary reason why a woman would find important among men in general the same attributes she does in a male partner; for example, it offers her no reproductive benefit for other men to be tough or place a high importance on sex. In fact, men possessing extreme degrees of these attributes could logically pose an increased threat to her survival or the survival of her children. In fact, several of the gendered partner-ideals supported by the factor analysis of the created measure offer no clear evolutionary benefit in themselves. Dominance and toughness could conceivably help with resource acquisition, and importance of sex could lead to a greater number of offspring, but the others dimensions' potential benefits are unclear. Ultimately, a more parsimonious explanation is that both women's masculinity ideology and their masculine partner-ideals reflect internalized cultural gender role norms. To the extent that a woman accepts traditional masculinity ideology, she is also more likely to apply those norms to her male romantic partners.

Masculine partner-perceptions. There were notable differences not only in the extent to which specific masculine partner-ideals were valued, but also in which specific masculine partner-perceptions described women's current partners. On average, women perceived their male partners as conforming less than desired to the norms of selfreliance through mechanical skills and dominance. But male partners were perceived as conforming more than desired for avoidance of femininity, toughness, importance of sex, and restrictive emotionality, as well as at the aggregate level across all seven dimensions. 
The variation among these dimensions raise questions for future research, including whether perceived conformity to certain masculine norms predict relationship satisfaction in a different directions (i.e., whether they are beneficial or detrimental) and what are the mechanisms driving these hypothetical associations. Post-hoc analyses reported in the previous chapter revealed that while aggregate masculine partnerperceptions had a relatively small, positive correlation with women's relationship satisfaction, individual dimensions varied in the direction of their association. Toughness, dominance, importance of sex, and self-reliance all had moderate, positive correlations. Self-reliance through mechanical skills, dominance, and importance of sex could be beneficial to romantic relationships through the respective provisions of instrumental support, relief of financial stress, and intimacy. It is less clear how toughness could operate in a positive manner. In contrast, results showed that restrictive emotionality had a moderate, negative correlation with relationship satisfaction. Following the work of Holmes (2014) on men's emotional control, this negative association likely reflects less communication than desired. Avoidance of femininity and negativity toward sexual minorities were not correlated with relationship satisfaction, perhaps because they are less germane to heterosexual relational behaviors.

These findings on masculine-perceptions and relationship satisfaction diverge from those reported in previous research. Burn and Ward (2005) found that all domains of masculine partner-perceptions, save pursuit of status, had significant, negative correlations with undergraduate women's relationship satisfaction. Holmes (2014) reported that all domains except risk taking had a negative association with relationship 
satisfaction. Neither of these studies identified a single masculine dimension that positively associated with relationship satisfaction. Some of this divergence in the pattern of results could be explained by differences in the exact characteristics rated; both of the previous studies utilized the dimensions of the Conformity to Masculine Norms Inventory (CMNI), which includes the dimensions of winning, emotional control, primacy of work, risk-taking, violence, heterosexual self-presentation, playboy, selfreliance, and power over women, rather than the MRNI-SF. However, there were significant areas of conceptual overlap between the CMNI and MRNI-SF — such as dominance, emotional control, and self-reliance — that would create the expectation of similar results.

More research is needed to clarify which masculine role norms, if any, have positive and negative associations with or causal effects on women's (and men's) relationship satisfaction and the mechanisms through which these domains operate. Holmes' (2014) work identifies some potential mechanisms, including decreased selfdisclosure and approaches to conflict.

Partner-ideal discrepancies. In the previous section, I explained that individual dimensions of masculine-perceptions diverged in the direction of their association with women's relationship satisfaction. However, as emphasized throughout this manuscript, conformity to gender role norms can potentially impact relationship satisfaction not only through their impact on relational behaviors, but also through their interaction with partner-ideals. Based on the logic of discrepancy theory (Higgins, 1987; 1989), gendered partner-ideal discrepancies should negatively predict relationship satisfaction. In other 
words, one will be less happy with the romantic relationship to the extent that one's partner is perceived as failing to fulfill one's gendered ideals. Supporting this concept, the results of Hypothesis 2 (path a) showed a negative association between aggregate masculine partner-ideal discrepancies and women's relationship satisfaction. The bivariate correlation expressing the same relationship is $r(342)=-.56, p<.001$ (presented as a correlation here because it is easier to interpret than unstandardized regression coefficients), showing that the constructs are strongly and negatively related.

These results offer further support for the idea that discrepancy theory (Higgins, $1987 ; 1989)$, which originally conceptualized in the domain of the self, is also applicable to romantic relationships. Discrepancy theory predicts that discrepancies between ideals and (perceptions of) reality should lead to psychological discomfort or negative affect (Higgins, 1987). The relational equivalent of these negative outcomes is relationship dissatisfaction. Work on the Ideal Standards Model (ISM) has already provided support for a negative association between partner-ideal discrepancies and relationship satisfaction using the three-dimension model of ideals (e.g., Fletcher et al., 1999). The present study extends this research to establish the relevance of gendered partner-ideal discrepancies to relationship satisfaction. In fact, the correlation between masculine-ideal discrepancies and relationship satisfaction $(r=-.56)$ has comparable strength to the correlation between ISM ideal-consistency and relationship quality $(r=.40)$ reported by Fletcher et al. (1999).

Here, I would like to clarify that the model of gendered partner-ideal discrepancies advanced in this manuscript is not mutually exclusive from non-gendered 
models of partner-ideal discrepancies such as the ISM. One model does not preclude the other. Instead, expanding previous partner-ideals research by examining explicitly gendered partner-ideal discrepancies can extend theory and suggest new applications. That being said, it would be interesting for future research to explore how gendered and non-gendered partner-ideals relate to each other and whether one area is more predictive of relationship outcomes (including, but not limited to, relationship satisfaction and IPV). It was interesting that the average masculine-ideal discrepancy found in the present study (-.48) is marginally stronger than the partner-ideal consistency coefficients reported by Fletcher et al. (1999). However, more research is needed to confirm whether masculineideal discrepancies are truly smaller than other partner-ideal discrepancies. What could explain this potential difference, and does it have any impact on relationship outcomes?

Discrepancy theory also makes predictions about the outcomes of discrepancies. Specifically, discomfort resulting from discrepancies can be mitigated in one of several ways: 1 . by changing reality to more closely match ideals, 2 . by changing perceptions of reality to more closely match ideals, or 3 . by making the ideal less mentally accessible. Applying this idea, work using the ISM has primarily focused on cognitive strategies for resolving discrepancies. However, the ISM states that the outcome of a discrepancy should depend on certain motives, namely relationship enhancement versus accuracy (Simpson et al., 2001). Cognitive strategies should be preferred by individuals motivated to idealize the relationship. In contrast, individuals motivated to hold accurate views of their partner should engage in more behavioral strategies such as attempting to change their partners or even leaving their relationships. This category of strategies has been 
neglected in prior research. The present study conceptualizes IPV as a strategy some individuals might use to influence their romantic partner to conform more closely to gendered partner-ideals. In Hypothesis $2 \mathrm{~b}$ of the present study, it was discovered that masculine-ideal discrepancies positively predict women's perpetration of emotional abuse. These results are discussed in greater detail below, but for now it will suffice to say that this finding supports the idea that individuals use a variety of different strategies (i.e., not just the cognitive ones examined in previous research) to address partner-ideal discrepancies. According to the ISM, it would be expected that women engaging in such behavioral strategies as emotional IPV perpetration are more strongly motivated to hold accurate views of their partners than they are to hold idealized views.

IPV. This study makes theoretical contributions to the study of the proximal predictors of IPV. First, it helps clarify the importance of masculinity ideology for IPV. As discussed in Chapter One, several researchers have argued that traditional gender ideology increases the risk of IPV perpetration when the role expectations inherent in that ideology are violated. For example, men with traditional gender ideology might perceive having a lower social status than their partners or failing to fulfill the breadwinner role as a loss of relational power and use IPV to regain that power (e.g., Anderson, 1997; Atkinson et al., 2005). However, past research has failed to assess these gendered expectations and perceptions directly. The present study has appropriately contextualized this line of reasoning within the work on partner-ideal discrepancies to assess this hypothesized mechanism directly. In other words, traditional gender ideology is important for IPV partly because it establishes gendered partner-ideals. When these 
partner-ideals are not fulfilled, the discrepancies can motivate IPV. Unfortunately, due to the measurement constraints discussed in Chapter Four, I was unable to test this model among men; only women's data could be used.

Hypothesis Two, which tested the association between gendered partner-ideal discrepancies and IPV perpetration, mediated by relationship satisfaction, was partially supported among women (it could not be tested in men). Masculine-ideal discrepancies, mediated by relationship satisfaction, negatively predicted women's emotional abuse perpetration $(\mathrm{H} 2 \mathrm{~b})$ but not physical assault or injury perpetration $(\mathrm{H} 2 \mathrm{a})$. This finding establishes the relevance of masculine-ideal discrepancies to women's emotional abuse perpetration against their male romantic partners. In other words, it provides support for the idea that some women use emotional abuse in reaction to low relationship satisfaction caused by masculine-ideal discrepancies. However, even for emotional abuse perpetration, the explained variance of the mediation model was small, $R^{2}=.07$. This fact may be a reflection of the complex etiology of IPV; there is evidence that many factors may contribute to IPV perpetration (and that IPV may have a variety of motivations, e.g., Bowen [2011]). However, as previously discussed, the purpose of Hypothesis Two was not to develop a new model of IPV. Rather, it was to explore IPV as a potential strategy to attempt to reduce gendered partner-ideal discrepancies.

The results of Hypothesis Two can be compared to the work of Jaspaert and Vervaeke (2014). Relevant to the present discussion, they tested a mediation model predicting psychological and physical IPV perpetration (using the CTS2 subscales) from general relationship-ideal discrepancies, mediated by relationship satisfaction, 
communication, and conflict resolution. Unfortunately, there is no point of comparison regarding injury perpetration, as Jaspaert and Vervaeke (2014) did not assess injury. In contrast to the results of the present project, Jaspaert and Vervaeke (2014) found a significant total, but not direct, effect of ideal discrepancies on physical assault perpetration. This suggests that the present study's lack of support for a similar model could reflect a failure to include the relevant mediators. However, I find it theoretically questionable to assume that ideal discrepancies lead to poor communication and poor conflict resolution (the additional mediators assessed by Jaspaert and Vervaeke [2014]); these mediators seem more accurately viewed as moderators. Mediators aside, differences in the results of the present study versus those of Jaspaert and Vervaeke (2014) could simply be a result of differences in the predictor variable. Not only was their measure of ideal discrepancies focusing on the relationship rather than the partner, but it was also general rather than focused on gendered attributes in particular. Perhaps individuals simply respond differently to general relationship-ideal discrepancies than they do to gendered partner-ideal discrepancies.

Also contrasting with the results of the present study, Jaspaert and Vervaeke (2014) found that neither the total nor the direct effect was significant for psychological violence perpetration. Their failure to find a relationship between ideal discrepancies and psychological IPV perpetration could be a result of several things. Maybe the relationship only exists for gendered partner-ideals, not general partner-ideals. Perhaps the MMEA is more sensitive to emotional abuse than is the CTS2 psychological subscale, or perhaps Jaspaert and Vervaeke's decision to dichotomize the subscales resulted in a 
lack of variance. Differences could also be due to the sample; their analyses included both partners in romantic couples, examined at the aggregate level without regard to gender. It is possible that the results found in Hypothesis Two of this study would not be found in men, so Jaspaert and Vervaeke's analysis could have failed to find significant results because it did not disaggregate by gender.

Returning to the differences in the results of $\mathrm{H} 2 \mathrm{a}$ and $\mathrm{H} 2 \mathrm{~b}$, there are several potential reasons why the model was supported for emotional abuse but not physical assault or injury. First, accepting the results of Hypothesis Two at face value, women who are dissatisfied as a result of masculine-ideal discrepancies could tend to respond with emotional control strategies rather than physical strategies. If this were true, it would have implications for intervention on women's IPV perpetration. Namely, women's perceptions of and reactions to masculine partner-ideal discrepancies could be an effective point of intervention for emotional but not physical IPV. Treatment could potentially involve helping women identify and replace their harmful emotionally abusive behaviors with healthier, more constructive ones.

Second, it is possible that differences in the measurement properties of the MMEA compared to the CTS2 caused the differences in support for H2a versus H2b. Prevalence rates of women's IPV perpetration were much higher on the MMEA than on either the CTS2 physical assault or injury subscales (75.3\% vs. $14.5 \%$ and $6.4 \%)$. It seems likely that emotional abuse perpetration truly is more common than physical abuse, given the lower social awareness of the problem and the difficulty of even defining the problem (partially because intent to harm is much harder to infer). It is also possible, 
though, that the MMEA is simply a more sensitive measure than the CTS2 subscales. Women who respond to partner-ideal discrepancies with abuse perpetration could be more likely to use relatively minor forms of abuse, such as "act[ing] cold or distant when angry" rather than more extreme actions such as "threaten[ing] to hit the other person." If so, then perhaps $\mathrm{H} 2 \mathrm{a}$ would have been supported if physical assault and injury had been assessed using a longer, more detailed assessment that contained more examples of less severe abuse, such as pinching or causing scratches or small bruises. However, even if such a measure existed, using it would have increased the already considerable time burden of participants in this study.

In addition to illuminating the relevance of gendered partner-ideal discrepancies to IPV, this study also contributes to research on the relation between gender role conformity and IPV. Tager et al. (2010) argued that masculine socialization can affect violence perpetration in at least two ways: 1 . directly through the adoption of attitudes supportive of violence and male power and 2. indirectly through emotion dysregulation. However, only a handful of studies have examined conformity to individual role norms; most measure global role conformity or conformity to stereotypically gendered traits. Of the studies that have measured individual gender roles, only one study sampled from the general population (i.e., Sears, 2011); the others recruited undergraduate students or known perpetrators of violence. Thus, the present study was useful in examining the accuracy and generalizability of past results.

The results of the tests of Hypothesis Three, which examined the associations between men's perceived gender role conformity and IPV perpetration (measured 
through women's reports of IPV victimization), showed that aggregate masculineperceptions had a small but significant association with emotional abuse. In other words, men perceived as generally more conforming to masculine roles perpetrated more emotional abuse. However, in contrast to past research, there was no evidence of such a relationship with physical assault or injury perpetration. As discussed in Chapter One, past research has found a positive link between men's overall masculine role conformity and both physical and verbal aggression (Cohn \& Zeichner, 2006; Sears, 2011; Tager et al., 2010). It is possible that the failure to find such a relationship in the present study was a result of dichotomizing the CTS2 subscales, which causes a loss of variance. Neither Tager et al. nor Sears (2011) dichotomized their respective IPV outcome variables: Tager et al. (2010) applied log-transformation, and Sears (2011) used raw scores. Additionally, they used different scales to assess aggression and IPV than those in the present study. Thus, it is also possible that the CTS2 physical assault and injury subscales are less sensitive to the specific violent behaviors truly related to masculine role conformity.

There were also some interesting findings at the level of conformity to specific male roles. Negativity toward sexual minorities had a small correlation with both injury and emotional abuse perpetration. Restrictive emotionality was positively correlated at a small magnitude with both physical assault and injury perpetration and at a medium magnitude with emotional abuse perpetration. All other correlations were nonsignificant.

It was somewhat surprising that negativity toward sexual minorities was positively related to IPV perpetration. Unfortunately, this finding cannot be interpreted 
in light of prior research, as neither Sears (2013) nor Tager et al. (2010) measured this construct. It is unclear from a theoretical perspective why negativity toward sexual minorities would be predictive of IPV in heterosexual romantic relationships. Perhaps negativity toward sexual minorities is reflective of some facet of personality that drives negative behaviors. Lending credence to this idea, some previous research has found a link between homophobic attitudes and destructive behaviors in romantic relationships: McGraw (2001) found that men who more strongly endorsed negativity toward sexual minorities on the MRNI were perceived by their female partners to be lower in both closeness-caregiving and openness of communication although not intrusiveness. However, it is unknown how directly men's masculinity ideology translates into their behaviors, let alone into their romantic partners' perceptions of their behavior in a given male role domain. Ultimately, more research is needed to confirm and clarify this finding.

The findings of Hypothesis Three regarding restrictive emotionality align with the results of Tager et al. (2010), who examined emotional control using the Conformity to Masculine Norms Inventory (CMNI) but not those of Sears (2011), who used the Masculinity Attitudes, Stress, and Conformity Questionnaire. It seems likely that this divergence is a result of the measurement differences across the studies. Specifically, the suppression of emotional vulnerability subscale used by Sears (2011) assesses not only emotional vulnerability — as its name would suggest—but also an emphasis on logic and rationality. As discussed in Chapter One, high levels of emotional restraint or suppression, which include avoiding negative emotions, could be expected to increase the risk of IPV perpetration by contributing to situations of conflict and reducing potential 
strategies for healthy conflict resolution (e.g., openly discussing negative feelings).

Logic and rationality, on the other hand, have no clear connection to IPV. Based on the findings of both the present study and Tager et al. (2010), it seems likely that restrictive emotionality—distinguished from valuing logic and rationality—-truly has a relationship with IPV perpetration.

It was surprising that neither dominance nor toughness were related to IPV perpetration in the present study, given that past research has supported similar constructs' association with violence (Sears, 2011; Tager et al., 2010). One possible reason is the relatively low internal consistency in the masculine-perceptions toughness subscale (which was just below the predetermined cutoff), which would have decreased the statistical power to detect a true correlation. Alternatively, these differences could be due to the source of the data. Data on conformity to masculine roles in the present study were partner-report, whereas both Sears (2011) and Tager et al. (2010) utilized self-report methods. This difference suggests two potential reasons for the divergence in the patterns of the present study's results from those of prior research. First, perhaps selfperceptions of masculine role conformity are more predictive of IPV, either because they are more accurate or because they have a mediating influence (e.g., perhaps seeing oneself as more dominant makes one more inclined to behave aggressively). Second, perhaps assessing masculine role conformity in the context of the romantic relationship qualitatively impacted women's responses. While only five of the 21 questions in the masculine-perceptions scale implicitly or explicitly refer to the romantic relationshipincluding two questions in the dominance subscale about family role and all three 
questions in the importance of sex subscale - it is possible that respondents interpreted all questions in the context of the romantic relationship. For example, "[My partner] isn't too quick to tell others that he cares about them" could be interpreted as applying to the romantic relationship or to relationships in general. If respondents were in fact interpreting all questions in the context of the romantic relationship, then perhaps dominance and toughness as measured in this study are different than masculine role conformity as measured in previous research. The goal of this project was to examine gender and violence dynamics within romantic relationships, rather than general gender role conformity. However, if romantic contextualization indeed caused the observed differences in results, it would be important to call out and account for in future research.

Disappointingly, this study was ultimately unable to be the first to examine relationships between conformity to individual feminine roles and IPV. While past research has generally supported a positive association between masculine role conformity and violence (Moore \& Stuart, 2005), much less is known about feminine role conformity. Identifying specific role norms as risk or protective factors would allow for better targeting in interventions and contribute to theory by suggesting potential mechanisms. For example, perhaps femininity has sometimes been found as protective against violence perpetration because certain role norms — such as caretaking — represent relational patterns that are incongruent with violence. While the present study was limited by measurement problems, the Conformity to Feminine Norms Inventory (CFNI, Parent \& Moradi, 2010) could be used to explore this issue. Unfortunately, the CFNI does not offer the benefits of one-to-one conceptual mapping onto the dimensions 
identified in the Femininity Ideology Scale (Lehman, 2000; Levant et al., 2007) or easy translation into feminine partner-ideals.

\section{Limitations}

It is important to recognize the limitations of the present study. Below, limitations are considered in the areas of study design, measures, data collection, processing, and analysis.

Study design. The design is correlational rather than experimental, precluding firm conclusions about causal relationships among the measured constructs of genderedideals, gendered-perceptions, relationship satisfaction, and IPV. This limitation is most important for the interpretation of the results for Hypothesis Two, which tests an implicitly causal model of ideal-discrepancies and relationship outcomes. Past longitudinal work using the ISM has established that partner-ideal discrepancies influence relationship satisfaction over time rather than the reverse (Campbell et al., 2001; Fletcher, Simpson, \& Thomas, 1998), supporting the directionality of the first pathway in the model. However, the rest of the model is based solely on theory. While discrepancy theory would predict that low relationship satisfaction should increase motivation to engage in controlling behaviors (such as IPV; Higgins, 1987; 1989), some past research on physical violence has suggested that physical IPV, at least, has a stronger impact on relationship satisfaction than the reverse. It is also possible that the two constructs are truly bidirectionally related. Unfortunately, the present study was unable to gather evidence of directionality, and the question remains to be answered by future research. 
Hypotheses One and Three also have implicit assumptions of causal directionality: gender ideology is assumed to influence gendered partner-ideals, and gender role conformity is assumed to lead to IPV perpetration. One could argue for the reverse. For example, perhaps women's masculinity ideology is informed by what they desire in their romantic partners, or perhaps men's perceived gender role conformity is in fact influenced by their abusive behaviors (given the results of Hypothesis Three, this idea would make sense for some domains, such as restrictive emotionality, but not others such as toughness or dominance). Also, not only is the reverse directionality possible, but it also possible that a third variable is influencing both factors in these hypotheses. Regarding Hypothesis One, perhaps socialization experiences independently influences both masculinity ideology and masculine-ideals.

An experimental design could provide evidence of causality for all the hypotheses examined in this study. However, given the nature of the constructs, an experimental design would be both impractical (e.g., how could one effectively change someone's gendered partner-ideals in an experimental manipulation?) and ethically questionable (i.e., experimentally manipulating IPV). Longitudinal designs would allow for stronger causal inference than was possible in this study by examining how change over time in some constructs predict change in others. Future research could also address concerns about mediating factors and third variables by replicating the research while including additional theoretically-relevant constructs such as relational power, control motivations, and personality dimensions linked to IPV. Several papers cited in this manuscript, such 
as Capaldi et al. (2012), Stith et al. (2004), and Ali and Naylor (2013a, 2013b) would provide fruitful sources of such constructs.

The need to limit participant burden also limited the ability of this study to illuminate the relationship between gender role conformity and IPV. As discussed in Chapter 1, conformity to particular role norms may be a better predictor of abusive or aggressive behaviors than gender identity. In other words, certain gendered patterns of behavior are linked to violence in both men and women. However, it was not feasible to collect data on conformity to the norms of both genders. Because of this limitation and problems with the feminine measures, it was not possible to draw conclusions about whether conformity to masculine and feminine role norms are risk factors for aggression in general; it was only possible to determine how men's masculinity (as reported by their partners) relates to IPV.

Measures. Another limitation involves construct measurement. Limitations of and problems with the measures of gendered partner-ideal and -perceptions created for this study have already been discussed. The Femininity Ideology Scale (FIS; Levant et al., 2007) also has potential limitations. Despite the evidence supporting the measure's validity, the Emotionality subscale contains a number of items with a concerning lack of face validity. A handful of items seem to belong in other domains. For example, "It is expected that a woman will engage in domestic hobbies such as sewing and decorating" sounds closer to the construct of stereotypic image and activities than to emotionality. It is possible that the Emotionality factor could have emerged as an artifact of item wording; all statements within this factor save one begin with the sentence stem "It is 
expected." Thus, these items could be measuring perceptions of wider social acceptance of traditional femininity ideology rather than personal endorsement.

There are also limitations regarding the measurement of IPV. For example, the CTS has been criticized for neglecting the motivation for and context of IPV (e.g., Dobash \& Dobash, 2004; further problematic aspects of the original CTS were discussed in Chapter 1). These same criticisms would apply to the CTS2. This study uses the MMEA as complement to the CTS2 in recognition of the fact that IPV can take nonphysical forms as well. While the inclusion of the MMEA contributes to a more complete picture of IPV than would be possible with the CTS2 subscales alone, all measures of IPV-related behaviors are vulnerable to social desirability influences and differences of interpretation. It is possible that participants were more reluctant to report physical assault and injury than emotional abuse, given their lesser social acceptability. This pattern of responding could explain why the pattern of results observed for Hypotheses Two and Three were stronger for emotional abuse than for physical assault and injury.

One measurement choice is not necessarily a limitation but represents a potential direction for future research. In Chapter 2, I argued why separate measures of gendered partner-ideals and -perceptions, compared using within-subjects correlations, were the best approach to measuring gendered partner-ideal discrepancies. However, some researchers have obtained meaningful results using a direct comparison measure that asks participants to assess the degree to which their partners match their ideals (Campbell et al., 2001, Lackenbauer \& Campbell, 2012, Overall et al., 2006). Lackenbauer and 
Campbell (2012) concluded that this approach leads to identical results when applied to the dimensions of the Ideal Standards Model. Eastwick et al. (2012) similarly found that this less common method of assessment yields comparable results to the pattern approach of within-subjects correlations. However, as proposed by Edwards (2001), it is possible that the "mental math" completed by participants answering direct comparison questions is qualitatively different than the calculations performed by the researchers on separate measures of ideals and discrepancies. More research is needed. If the methods were determined to be equivalent, then direct comparison items would be advantageous when efficiency is more important than the ability to separately examine ideals and perceptions. If the methods are not equivalent, however, it raises questions on the cognitive processes participants use to answer direct comparison items and which method more accurately reflects the processes described by Discrepancy Theory.

Sample and data collection. A last major limitation involves the sample and data collection procedure. The decision was made to recruit through MTurk because past research has shown that the platform allows researchers to access more demographically diverse and representative samples than is possible in most American college student samples without sacrificing data reliability (Buhrmester, Kwang, \& Gosling, 2011). To support the generalizability of results, it is important to capture a range of gender ideologies, demographic characteristics, and relationship lengths, as these factors are associated with relationship satisfaction and rates of IPV. However, it is possible that some participants provided fraudulent data. Additionally, differences in relationship satisfaction or experience with abusive behaviors could drive attrition, resulting in 
sampling bias. The consent form was designed to avoid revealing that the study focused on IPV, but participants were free to quit the study for any reason including discomfort with the content of the questions. Only $2.0 \%$ of qualifying participants who began the study dropped out before finishing. Most attrition was early in the questionnaire, reducing the likely impact of sampling bias on results. Given the eligibility requirements for participation, it is also unknown whether results generalize to other samples including non-heterosexual couples, couples that have been together less than six months, and couples in other cultures.

Analysis. In Chapter One, I explained how our understanding of IPV is being deepened through the use of dyadic analysis. Unfortunately, an analysis of dyadic IPV data is beyond the scope of this study due to a limited sample size and measurement problems (i.e., problems with the feminine measures make it inadvisable to model men's feminine-ideals and -perceptions in conjunctions with the masculine-ideals and perceptions of their partners). However, such an examination would be informative in its ability to identify different patterns of IPV. Dyadic analysis could be used, for example, to determine how Johnson's $(1995,2006)$ typology of IPV factors into the models examined in this manuscript.

A second limitation of this project's analysis strategy involves the level of granularity used in testing Hypothesis Two. A logical extension of Mahalik et al.'s (2005) call to treat gender roles as multidimensional is to examine elements of masculine-ideal discrepancies separately. However, the present study did not examine the association between individual domains of masculine-ideal discrepancies and 
women's relationship satisfaction. I chose to examine masculine-ideal discrepancies at the aggregate level only because there was insufficient past research on masculine-ideal discrepancies to make clear predictions about the strength or directionality of effects. However, now that there exists a viable measure of masculine-ideal discrepancies, it would be interesting to examine these patterns of associations. As discussed earlier, there are differences in the directionality of the correlations between specific masculine partner-perceptions and women's relationship satisfaction. It is also possible that some masculine-ideal discrepancies have a stronger association with relationship satisfaction. For example, discrepancies in the domains of restrictive emotionality and importance of sex could have a strong association because they directly impact the relational behaviors of disclosure and intimacy, respectively. Future research on gendered partner-ideal discrepancies should continue this line of inquiry.

\section{Conclusions}

The present study represents many "firsts": it was the first to examine gendered partner-ideals at the level of specific role norms, the first to examine masculine-ideal discrepancies as a predictor of women's physical IPV and emotional control behaviors (although one study has examined relationship-ideal discrepancies and IPV; Jaspaert \& Vervaeke, 2014), and one of the first to examine the relationship between conformity to individual masculine norms with IPV. Results showed that women's endorsement of specific masculine partner-ideals mirrors their endorsement of specific dimensions of masculinity ideology. It was discovered that masculine-ideal discrepancies predict women's emotional abuse perpetration, mediated by relationship satisfaction. While 
inconsistent across male role dimensions and types of IPV, there was also limited evidence for a link between men's perceived conformity to male roles and their IPV perpetration. Unfortunately, the hypotheses utilizing men's data could not be tested due to problems with the new measures of feminine-ideals and -perceptions.

Despite this study's limitations, it represents much-needed advancement toward understanding the antecedents, structure, and potential outcomes of gendered partnerideals within romantic relationships. The integration of theory from the literatures on gender and romantic relationships creates the opportunity for new insights into both traditions, and the method addresses a need for consideration of interactive effects in relationship research (Simpson et al., 2001). In addition to its theoretical contributions, the present study could inspire advancements in applied work. For example, results could be used to identify couples at high risk for IPV based on women's masculine-ideal discrepancies or masculine-perceptions. Discrepancy theory could be used as a framework to intervene in couples already exhibiting abuse by encouraging individuals to critically examine their gendered partner-ideals and reactions to ideal discrepancies. 


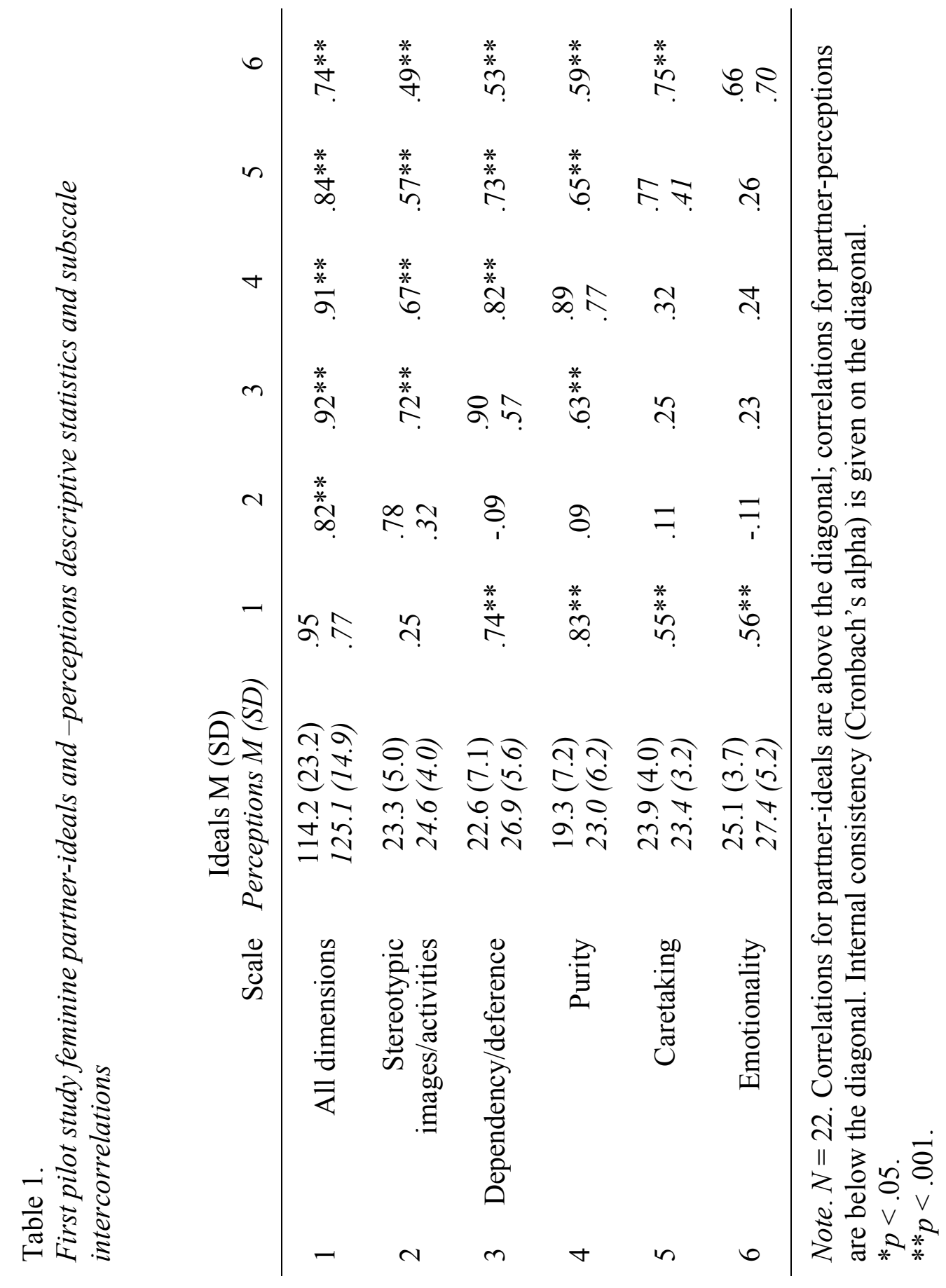




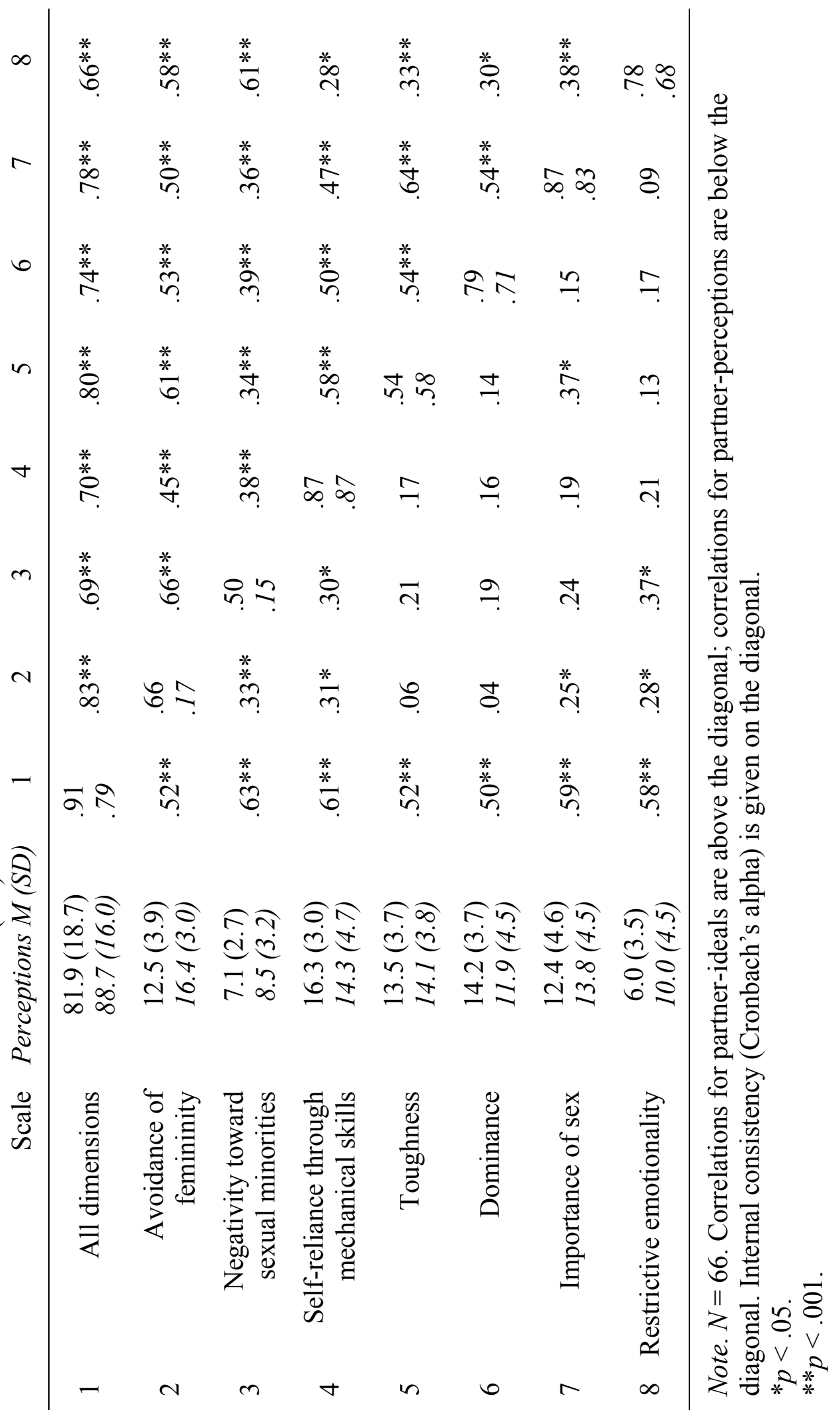


Table 3.

First pilot study Hypothesis 2: Correlations between partner-ideal discrepancies and relationship satisfaction

Male participants

\begin{tabular}{rcc}
\hline & $\mathrm{M}(S D)$ & $r$ \\
\hline Feminine partner- & -.49 & -- \\
ideal discrepancies & $(.21)$ & - \\
RDAS total & 51.73 & $-.67^{* *}$ \\
& $(7.08)$ & \\
RDAS - Consensus & 23.55 & $-.58^{*}$ \\
& $(3.14)$ & \\
RDAS - Satisfaction & 15.32 & $-.69^{* *}$ \\
& $(2.68)$ & \\
RDAS - Cohesion & 12.86 & $-.44^{*}$ \\
& $(2.56)$ & \\
RAS total & 4.18 & $-.59^{*}$ \\
& $(.69)$ &
\end{tabular}

Female participants

\begin{tabular}{rcc}
\hline & $\mathrm{M}(\mathrm{SD})$ & $r$ \\
\hline Masculine partner- & -.48 & -- \\
ideal discrepancies & $(.29)$ & - \\
RDAS total & 49.91 & $-.55^{* *}$ \\
& $(8.12)$ & \\
RDAS- Consensus & 22.46 & $-.46^{* *}$ \\
& $(3.70)$ & \\
& 14.83 & $-.50^{* *}$ \\
RDAS - Satisfaction & $(3.22)$ & \\
& 12.62 & $-.40^{* *}$ \\
RDAS - Cohesion & $(3.02)$ & \\
& 4.21 & $-.50^{* *}$ \\
RAS total & $(.80)$ &
\end{tabular}

Note. $N=22$ men; 69 women. RDAS total = aggregate Revised Dyadic Adjustment Scale score; RAS total = aggregate Relationship Assessment Scale score.

$* p<.05$. $* * p<.001$. 


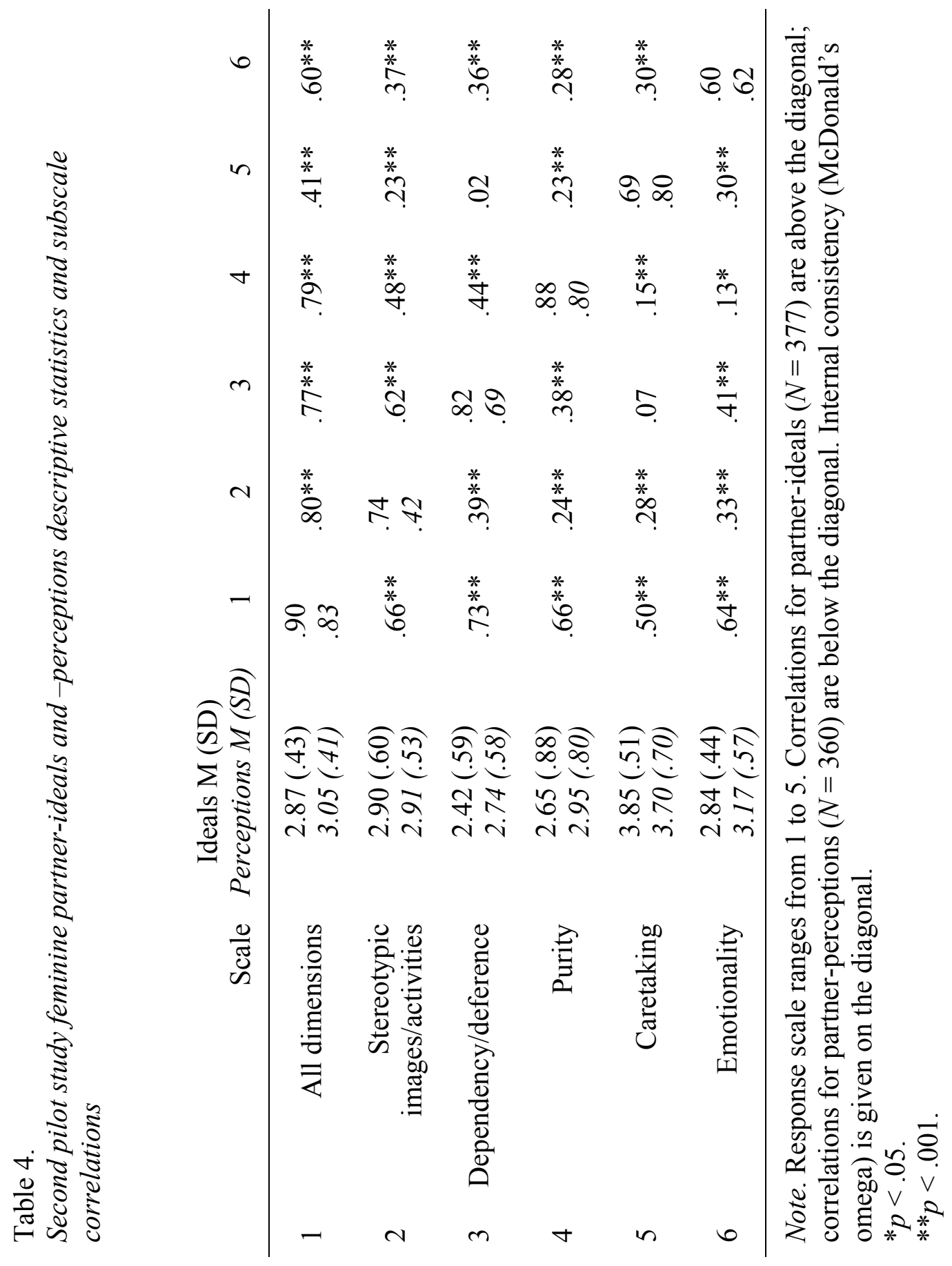


Table 5.

Second pilot study feminine partner-ideals and-perceptions factor loadings

\begin{tabular}{|c|c|c|c|c|c|c|c|c|c|}
\hline \multirow[b]{2}{*}{ Factor } & \multirow[b]{2}{*}{ Item \# } & \multicolumn{4}{|c|}{ Feminine ideals } & \multicolumn{4}{|c|}{ Feminine perceptions } \\
\hline & & Loading & SE & $p$ & $\begin{array}{c}\text { Std. } \\
\text { loading }\end{array}$ & Loading & SE & $p$ & $\begin{array}{c}\text { Std. } \\
\text { Loading }\end{array}$ \\
\hline \multirow{9}{*}{$\begin{array}{r}\text { Stereotypic } \\
\text { images and } \\
\text { activities }\end{array}$} & 19 & .73 & .07 & $<.001$ & .59 & .45 & .07 & $<.001$ & .37 \\
\hline & 23 & .58 & .06 & $<.001$ & .52 & 1.00 & .07 & $<.001$ & .79 \\
\hline & 27 & .64 & .06 & $<.001$ & .57 & .18 & .06 & .003 & .19 \\
\hline & 28 & .57 & .05 & $<.001$ & .57 & .49 & .07 & $<.001$ & .42 \\
\hline & 29 & .40 & .06 & $<.001$ & .40 & -.34 & .08 & $<.001$ & -.27 \\
\hline & 31 & .38 & .05 & $<.001$ & .40 & .26 & .07 & $<.001$ & .21 \\
\hline & $41 \mathrm{a}$ & .45 & .06 & $<.001$ & .45 & .08 & .07 & .31 & .06 \\
\hline & 43 & .45 & .06 & $<.001$ & .45 & .78 & .06 & $<.001$ & .72 \\
\hline & 45 & .43 & .05 & $<.001$ & .46 & .06 & .07 & .43 & .05 \\
\hline \multirow{11}{*}{$\begin{array}{l}\text { Dependency/ } \\
\text { deference }\end{array}$} & $1 \mathrm{a}$ & .55 & .06 & $<.001$ & .52 & .38 & .08 & $<.001$ & .29 \\
\hline & 3 & .26 & .05 & $<.001$ & .29 & .15 & .09 & .09 & .10 \\
\hline & 4 & .53 & .05 & $<.001$ & .53 & .54 & .08 & $<.001$ & .39 \\
\hline & 6 & .73 & .05 & $<.001$ & .70 & .58 & .06 & $<.001$ & .53 \\
\hline & 8 & .32 & .05 & $<.001$ & .36 & .17 & .08 & .03 & .13 \\
\hline & 9 & .57 & .05 & $<.001$ & .59 & .49 & .06 & $<.001$ & .48 \\
\hline & 10 & .40 & .05 & $<.001$ & .40 & .38 & .07 & $<.001$ & .32 \\
\hline & 11 & .62 & .06 & $<.001$ & .55 & .53 & .07 & $<.001$ & .47 \\
\hline & 13 & .40 & .05 & $<.001$ & .43 & .54 & .06 & $<.001$ & .49 \\
\hline & 16 & .77 & .05 & $<.001$ & .74 & .83 & .07 & $<.001$ & .70 \\
\hline & 18 & .66 & .05 & $<.001$ & .62 & .47 & .06 & $<.001$ & .43 \\
\hline \multirow{9}{*}{ Purity } & 5 & .73 & .07 & $<.001$ & .52 & .47 & .07 & $<.001$ & .37 \\
\hline & 12 & .88 & .06 & $<.001$ & .69 & .87 & .07 & $<.001$ & .67 \\
\hline & 15 & .95 & .06 & $<.001$ & .73 & .77 & .07 & $<.001$ & .58 \\
\hline & 22 & .91 & .06 & $<.001$ & .68 & .81 & .08 & $<.001$ & .57 \\
\hline & 25 & 1.04 & .06 & $<.001$ & .82 & .93 & .08 & $<.001$ & .67 \\
\hline & 26 & .84 & .05 & $<.001$ & .79 & .95 & .07 & $<.001$ & .72 \\
\hline & 30 & .85 & .05 & $<.001$ & .74 & .85 & .07 & $<.001$ & .65 \\
\hline & 32 & .53 & .06 & $<.001$ & .45 & .32 & .08 & $<.001$ & .23 \\
\hline & 35 & .72 & .05 & $<.001$ & .67 & .49 & .06 & $<.001$ & .46 \\
\hline \multirow{7}{*}{ Caretaking } & 2 & .53 & .04 & $<.001$ & .68 & .76 & .05 & $<.001$ & .77 \\
\hline & 7 & .46 & .04 & $<.001$ & .67 & .74 & .04 & $<.001$ & .81 \\
\hline & 14 & .45 & .05 & $<.001$ & .52 & .76 & .05 & $<.001$ & .71 \\
\hline & 17 & .34 & .06 & $<.001$ & .34 & .53 & .06 & $<.001$ & .50 \\
\hline & 21 & .44 & .04 & $<.001$ & .59 & .65 & .05 & $<.001$ & .66 \\
\hline & 24 & .34 & .05 & $<.001$ & .40 & .39 & .06 & $<.001$ & .37 \\
\hline & 38 & .39 & .06 & $<.001$ & .40 & .53 & .06 & $<.001$ & .48 \\
\hline \multirow{9}{*}{ Emotionality } & 20 & .27 & .06 & $<.001$ & .28 & .86 & .07 & $<.001$ & .71 \\
\hline & 33 & .25 & .06 & $<.001$ & .28 & .39 & .06 & $<.001$ & .37 \\
\hline & 34 & -.01 & .06 & .89 & -.01 & .43 & .06 & $<.001$ & .39 \\
\hline & 36 & .42 & .06 & $<.001$ & .44 & .27 & .08 & $<.001$ & .22 \\
\hline & 37 & .65 & .05 & $<.001$ & .71 & .08 & .08 & .25 & .07 \\
\hline & 39 & .37 & .05 & $<.001$ & .43 & .23 & .07 & $<.001$ & .21 \\
\hline & 40 & -.01 & .06 & .90 & -.01 & .38 & .06 & $<.001$ & .38 \\
\hline & 42 & .29 & .06 & $<.001$ & .32 & .45 & .07 & $<.001$ & .37 \\
\hline & 44 & .08 & .06 & .18 & .09 & .70 & .06 & $<.001$ & .68 \\
\hline
\end{tabular}


Table 6.

Second pilot study feminine partner-ideals and-perceptions latent factor correlations

\begin{tabular}{lllllll} 
& & 1 & 2 & 3 & 4 & 5 \\
\hline 1 & Stereotypic image and activities & -- & $.78^{* *}$ & $.55^{* *}$ & $.18^{*}$ & $.63^{* *}$ \\
2 & Dependence/deference & .32 & - & $.49^{* *}$ & -.11 & $.78^{* *}$ \\
3 & Purity & .18 & $.48^{* *}$ & -- & .10 & $.48^{* *}$ \\
4 & Caretaking & .38 & .12 & .08 & -- & -.13 \\
5 & Emotionality & .34 & $.32^{* *}$ & .04 & $.56^{* *}$ & -- \\
\hline
\end{tabular}

Note. Correlations for partner-ideals $(N=377)$ are above the diagonal; correlations for partner-perceptions $(N=360)$ are below the diagonal.

$* p<.05$.

$* * p<.001$. 


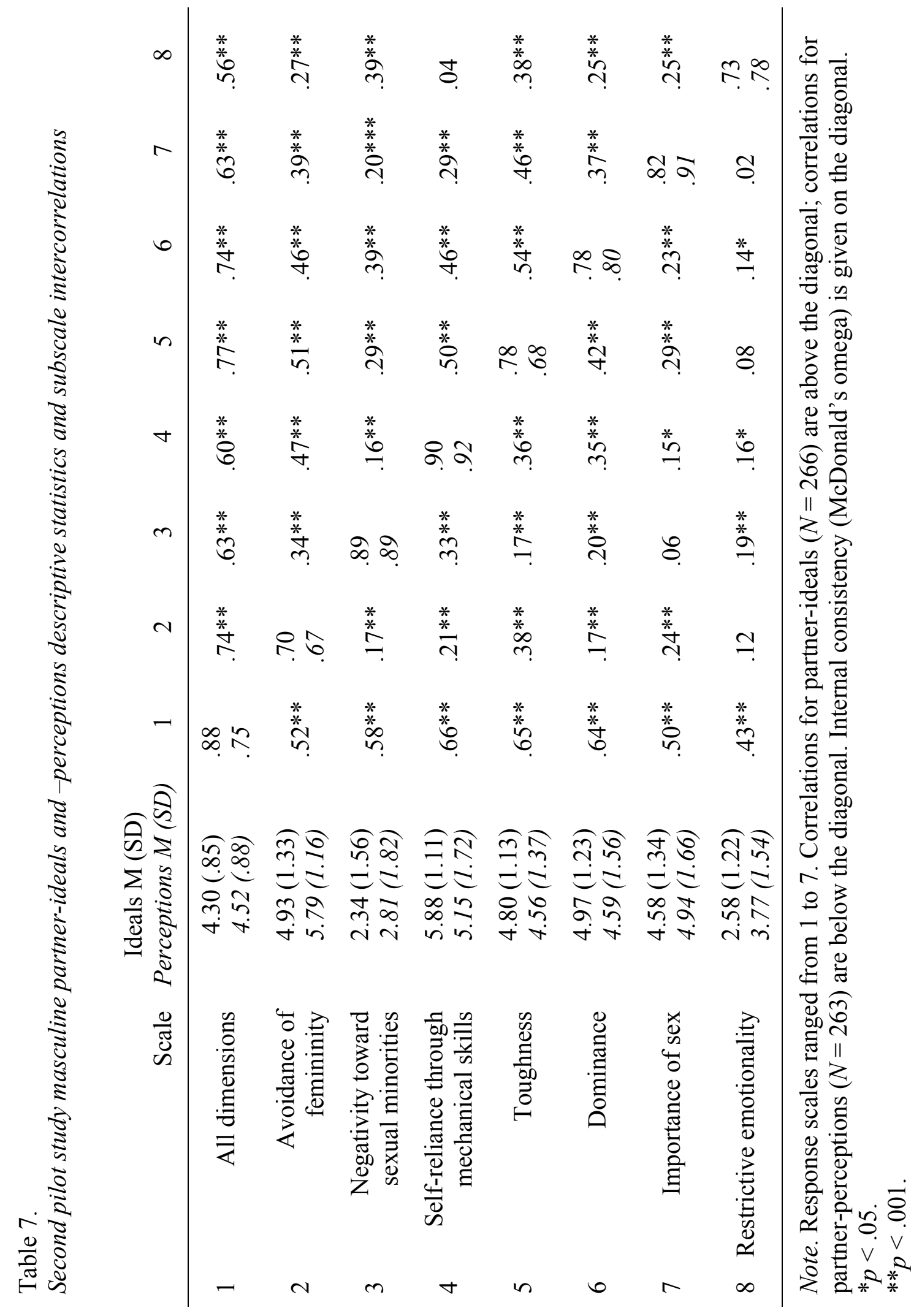


Table 8.

Second pilot study masculine partner-ideals and-perceptions factor loadings

\begin{tabular}{|c|c|c|c|c|c|c|c|c|c|}
\hline \multirow[b]{2}{*}{ Factor } & \multicolumn{5}{|c|}{ Masculine ideals } & \multicolumn{4}{|c|}{ Masculine perceptions } \\
\hline & Item \# & Loading & SE & $p$ & $\begin{array}{c}\text { Std. } \\
\text { Loading }\end{array}$ & Loading & SE & $p$ & $\begin{array}{c}\text { Std. } \\
\text { Loading }\end{array}$ \\
\hline \multirow{3}{*}{$\begin{array}{r}\text { Avoidance of } \\
\text { femininity }\end{array}$} & 4 & 1.23 & .11 & $<.001$ & .67 & 1.08 & .15 & $<.001$ & .54 \\
\hline & 8 & 1.04 & .10 & $<.001$ & .65 & 1.15 & .12 & $<.001$ & .78 \\
\hline & $10 \mathrm{a}$ & 1.05 & .10 & $<.001$ & .65 & .54 & .09 & $<.001$ & .44 \\
\hline Negativity & $1 \mathrm{~b}$ & 1.51 & .10 & $<.001$ & .79 & 1.89 & .11 & $<.001$ & .86 \\
\hline \multirow{2}{*}{$\begin{array}{r}\text { toward sexual } \\
\text { minorities }\end{array}$} & $5 \mathrm{a}$ & 1.21 & .08 & $<.001$ & .77 & 1.28 & .10 & $<.001$ & .74 \\
\hline & 13 & 1.73 & .09 & $<.001$ & .97 & 2.01 & .11 & $<.001$ & .94 \\
\hline \multirow{3}{*}{ Self-reliance } & 6 & 1.07 & .06 & $<.001$ & .93 & 1.64 & .08 & $<.001$ & .95 \\
\hline & 7 & 1.06 & .06 & $<.001$ & .93 & 1.70 & .08 & $<.001$ & .96 \\
\hline & 14 & 1.03 & .07 & $<.001$ & .76 & 1.61 & .11 & $<.001$ & .79 \\
\hline \multirow{3}{*}{ Toughness } & 19 & .57 & .07 & $<.001$ & .49 & 1.18 & .10 & $<.001$ & .76 \\
\hline & $20 \mathrm{a}$ & 1.32 & .10 & $<.001$ & .79 & 1.09 & .12 & $<.001$ & .60 \\
\hline & $20 \mathrm{~b}$ & .99 & .09 & $<.001$ & .66 & .99 & .13 & $<.001$ & .52 \\
\hline \multirow{3}{*}{ Dominance } & 2 & .59 & .09 & $<.001$ & .41 & .94 & .11 & $<.001$ & .51 \\
\hline & $3 b$ & 1.28 & .09 & $<.001$ & .82 & 1.59 & .10 & $<.001$ & .86 \\
\hline & 12 & 1.42 & .08 & $<.001$ & .90 & 1.64 & .11 & $<.001$ & .84 \\
\hline \multirow{3}{*}{$\begin{array}{r}\text { Importance of } \\
\text { sex }\end{array}$} & 9 & 1.20 & .09 & $<.001$ & .76 & 1.52 & .09 & $<.001$ & .86 \\
\hline & 11 & 1.15 & .09 & $<.001$ & .74 & 1.52 & .09 & $<.001$ & .85 \\
\hline & 18 & 1.28 & .09 & $<.001$ & .82 & 1.70 & .09 & $<.001$ & .94 \\
\hline \multirow{3}{*}{$\begin{array}{r}\text { Restrictive } \\
\text { emotionality }\end{array}$} & 15 & 1.12 & .08 & $<.001$ & .82 & 1.53 & .11 & $<.001$ & .84 \\
\hline & 16 & 1.10 & .09 & $<.001$ & .71 & 1.43 & .12 & $<.001$ & .74 \\
\hline & 21 & .91 & .10 & $<.001$ & .56 & 1.10 & .11 & $<.001$ & .61 \\
\hline
\end{tabular}


Table 9.

Second pilot study masculine partner-ideals and-perceptions latent factor correlations

\begin{tabular}{|c|c|c|c|c|c|c|c|c|}
\hline & & 1 & 2 & 3 & 4 & 5 & 6 & 7 \\
\hline 1 & $\begin{array}{l}\text { Avoidance of } \\
\text { femininity }\end{array}$ & -- & $.42 * *$ & $.57 * *$ & $.62 * *$ & $.71 * *$ & $.52 * *$ & $.35 * *$ \\
\hline 2 & $\begin{array}{l}\text { Negativity toward } \\
\text { sexual minorities }\end{array}$ & $.19 *$ & -- & $.16^{*}$ & $.43 * *$ & $.37 * *$ & $.21 *$ & $.49 * *$ \\
\hline 3 & $\begin{array}{l}\text { Self-reliance through } \\
\text { mechanical skills }\end{array}$ & $.27 *$ & $.35 * *$ & -- & $.52 * *$ & $.56^{* *}$ & $.31 * *$ & .04 \\
\hline 4 & Dominance & $.19^{*}$ & $.28 * *$ & $.41 * *$ & -- & $.64 * *$ & $.46^{* *}$ & $.27 * *$ \\
\hline 5 & Toughness & $.45^{* *}$ & $.21 *$ & $.49 * *$ & $.61 * *$ & -- & $.57 * *$ & $.50 * *$ \\
\hline 6 & Importance of sex & $.26^{*}$ & .06 & .13 & $.27 * *$ & $.38 * *$ & -- & $.29 * *$ \\
\hline 7 & $\begin{array}{l}\text { Restrictive } \\
\text { emotionality }\end{array}$ & .16 & $.22 *$ & .14 & $.16^{*}$ & .01 & .02 & -- \\
\hline
\end{tabular}

Note. Correlations for partner-ideals $(N=284)$ are above the diagonal; correlations for partner-perceptions $(N=266)$ are below the diagonal.

$* p<.05$.

$* * p<.001$. 


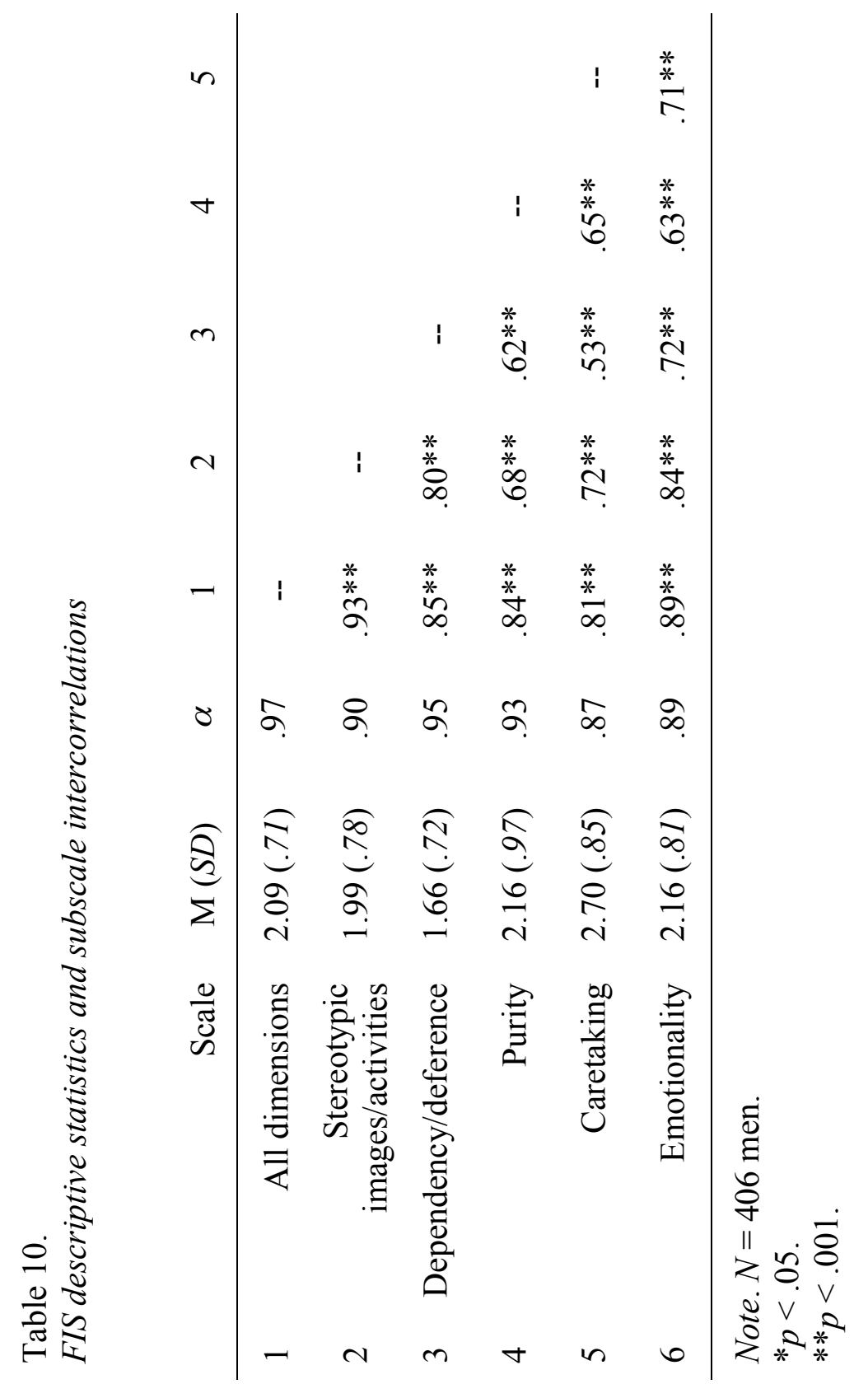




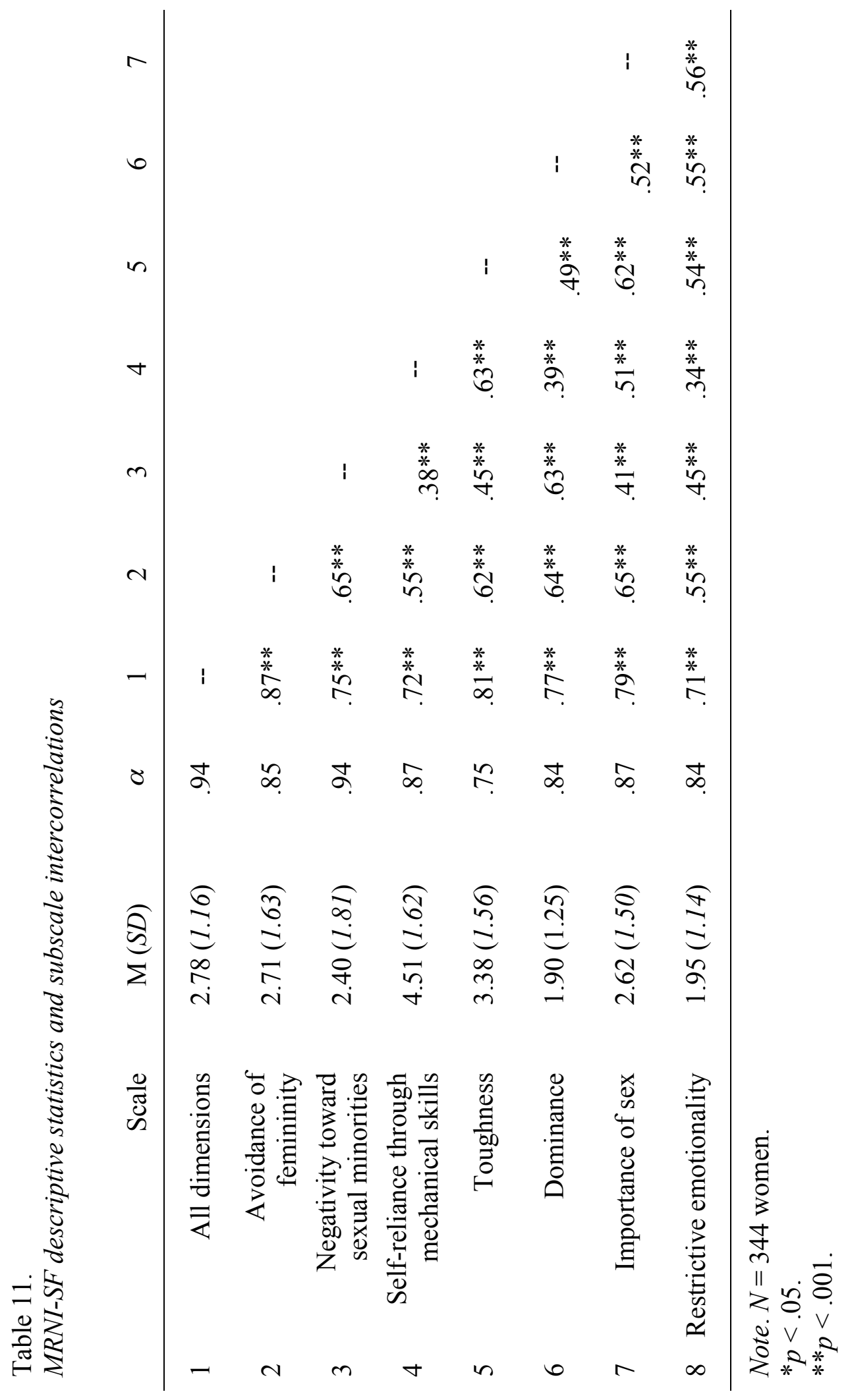




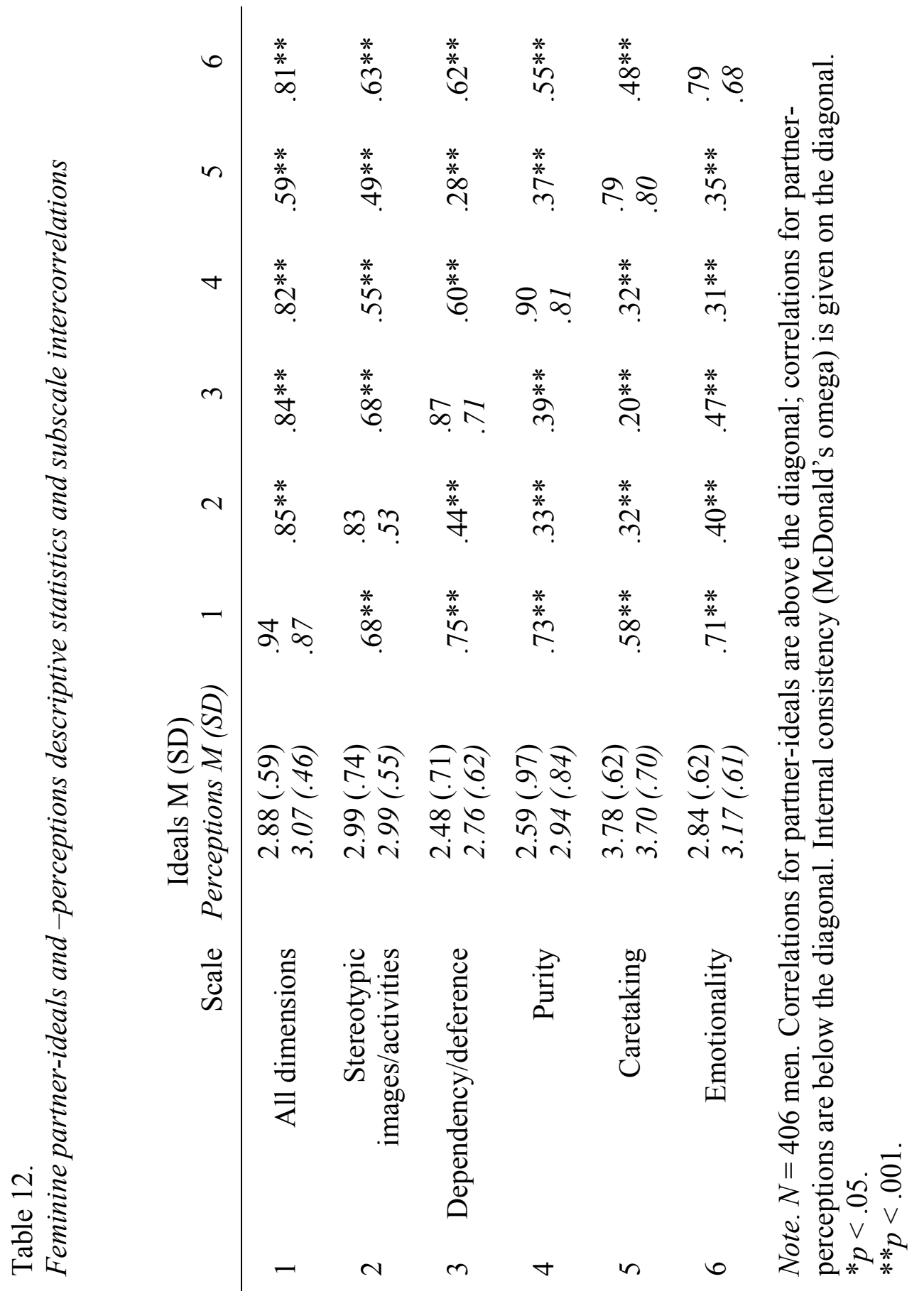




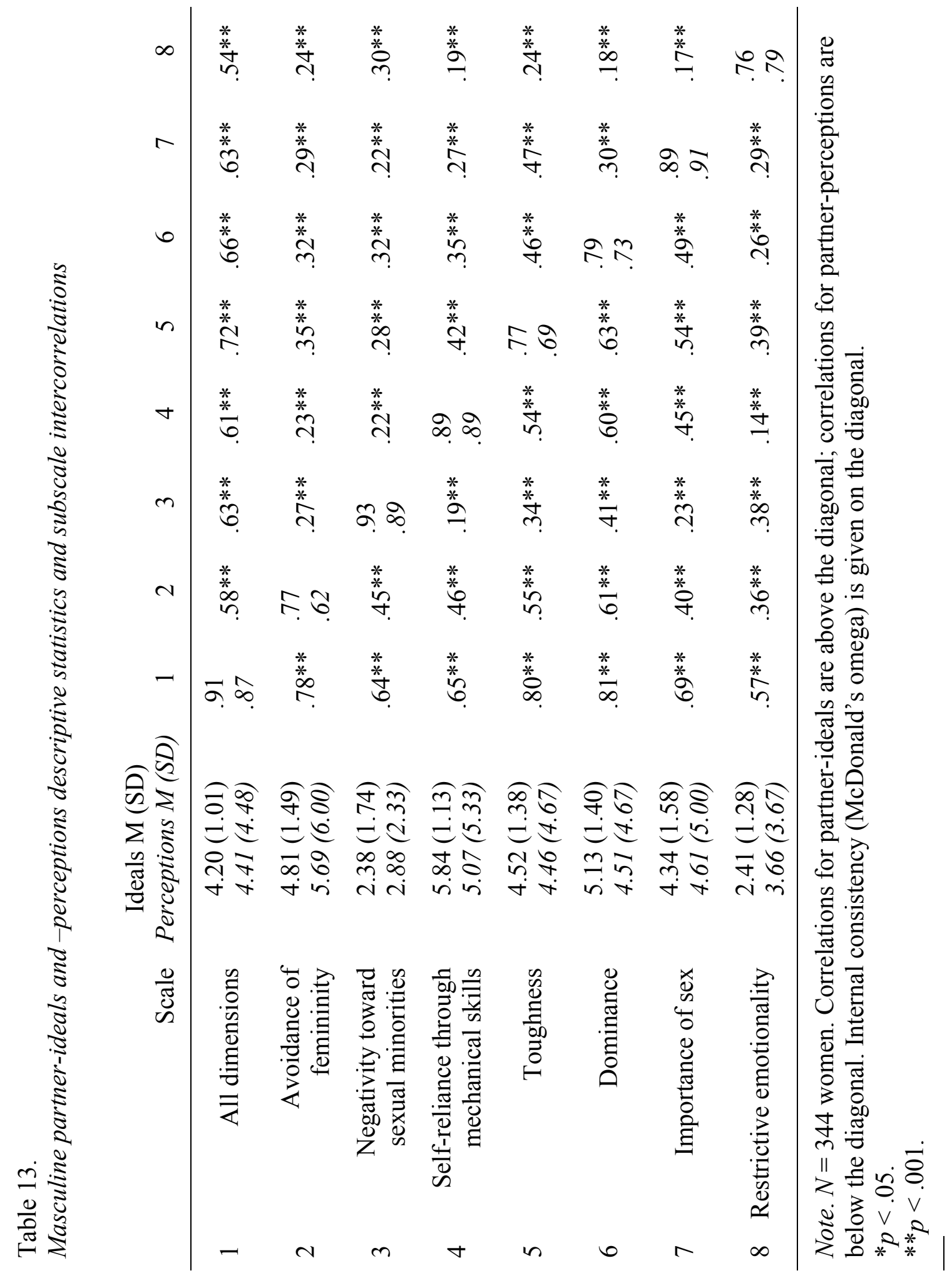




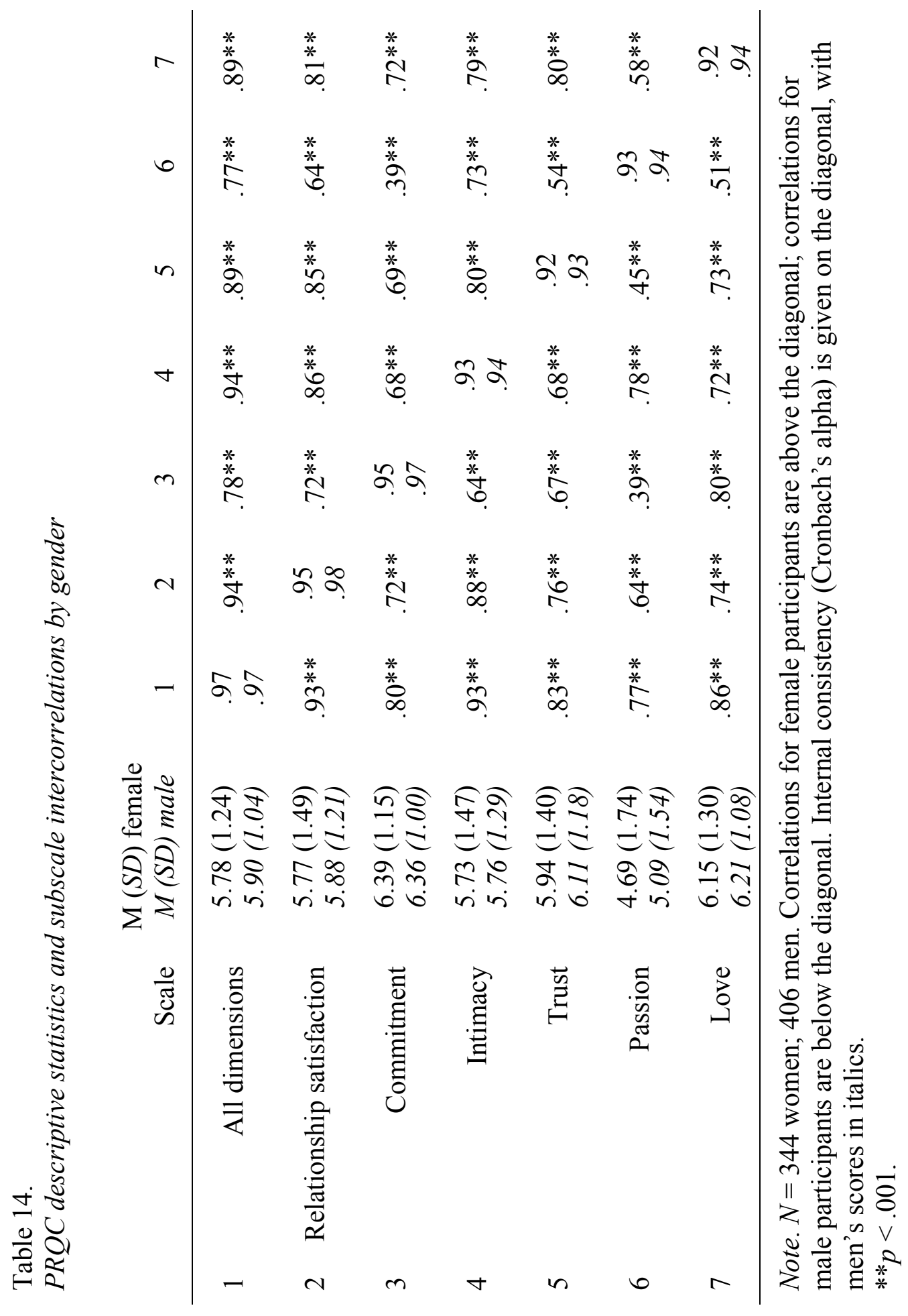


Table 15.

CTS2 descriptive statistics and subscale intercorrelations for female participants

\begin{tabular}{|c|c|c|c|c|c|c|c|c|}
\hline & Scale & $\begin{array}{c}\mathrm{M} \\
(S D)\end{array}$ & Prevalence & $\begin{array}{c}\text { Chronicity } \\
\text { M } \\
\text { (SD) }\end{array}$ & 1 & 2 & 3 & 4 \\
\hline 1 & $\begin{array}{r}\text { Physical } \\
\text { assault } \\
\text { perpetration }\end{array}$ & $\begin{array}{c}3.19 \\
(19.27)\end{array}$ & 14.5 & $\begin{array}{c}21.94 \\
(46.69)\end{array}$ & .96 & & & \\
\hline 2 & $\begin{array}{r}\text { Injury } \\
\text { perpetration }\end{array}$ & $\begin{array}{c}1.44 \\
(10.44)\end{array}$ & 6.4 & $\begin{array}{c}22.50 \\
(35.83)\end{array}$ & $.96 * *$ & .90 & & \\
\hline 3 & $\begin{array}{r}\text { Physical } \\
\text { assault } \\
\text { victimization }\end{array}$ & $\begin{array}{c}4.03 \\
(23.79)\end{array}$ & 16.9 & $\begin{array}{c}23.93 \\
(54.05)\end{array}$ & $.89 * *$ & $.94 * *$ & .96 & \\
\hline 4 & $\begin{array}{r}\text { Injury } \\
\text { victimization }\end{array}$ & $\begin{array}{c}1.33 \\
(9.20) \\
\end{array}$ & 8.7 & $\begin{array}{c}15.20 \\
(27.97)\end{array}$ & $.88 * *$ & $.83 * *$ & $.84 * *$ & .90 \\
\hline
\end{tabular}

Note. $N=344$. Internal consistency (Cronbach's alpha) is given on the diagonal. All reported numbers use raw scores for ease of interpretation and comparability to past research. Prevalence refers to the percentage of women reporting at least one incident in the past six months. Chronicity statistics reflect the frequency of violent incidents among women reporting at least one such incident in the past six months.

$* * p<.001$. 
Table 16.

CTS2 descriptive statistics and subscale intercorrelations for male participants

\begin{tabular}{|c|c|c|c|c|c|c|c|c|}
\hline & Scale & $\begin{array}{c}\mathrm{M} \\
(S D)\end{array}$ & Prevalence & $\begin{array}{l}\text { Chronicity } \\
\text { M } \\
\text { (SD) }\end{array}$ & 1 & 2 & 3 & 4 \\
\hline 1 & $\begin{array}{r}\text { Physical } \\
\text { assault } \\
\text { perpetration }\end{array}$ & $\begin{array}{c}2.35 \\
(13.34)\end{array}$ & 13.1 & $\begin{array}{c}18.00 \\
(33.15)\end{array}$ & .91 & & & \\
\hline 2 & $\begin{array}{r}\text { Injury } \\
\text { perpetration }\end{array}$ & $\begin{array}{c}.84 \\
(5.94)\end{array}$ & 5.2 & $\begin{array}{c}16.24 \\
(21.25)\end{array}$ & $.64 * *$ & .82 & & \\
\hline 3 & $\begin{array}{r}\text { Physical } \\
\text { assault } \\
\text { victimization }\end{array}$ & $\begin{array}{c}2.72 \\
(13.41)\end{array}$ & 15.0 & $\begin{array}{c}18.12 \\
(30.51)\end{array}$ & $.83 * *$ & $.78 * *$ & .87 & \\
\hline 4 & $\begin{array}{r}\text { Injury } \\
\text { victimization }\end{array}$ & $\begin{array}{c}.96 \\
(8.63)\end{array}$ & 4.7 & $\begin{array}{c}20.57 \\
(35.33)\end{array}$ & $.89 * *$ & $.60 * *$ & $.71 * *$ & .95 \\
\hline
\end{tabular}

Note. $N=406$. Internal consistency (Cronbach's alpha) is given on the diagonal. All reported numbers use raw scores for ease of interpretation and comparability to past research. Prevalence refers to the percentage of men reporting at least one incident in the past six months. Chronicity statistics reflect the frequency of violent incidents among men reporting at least one such incident in the past six months.

$* * p<.001$. 


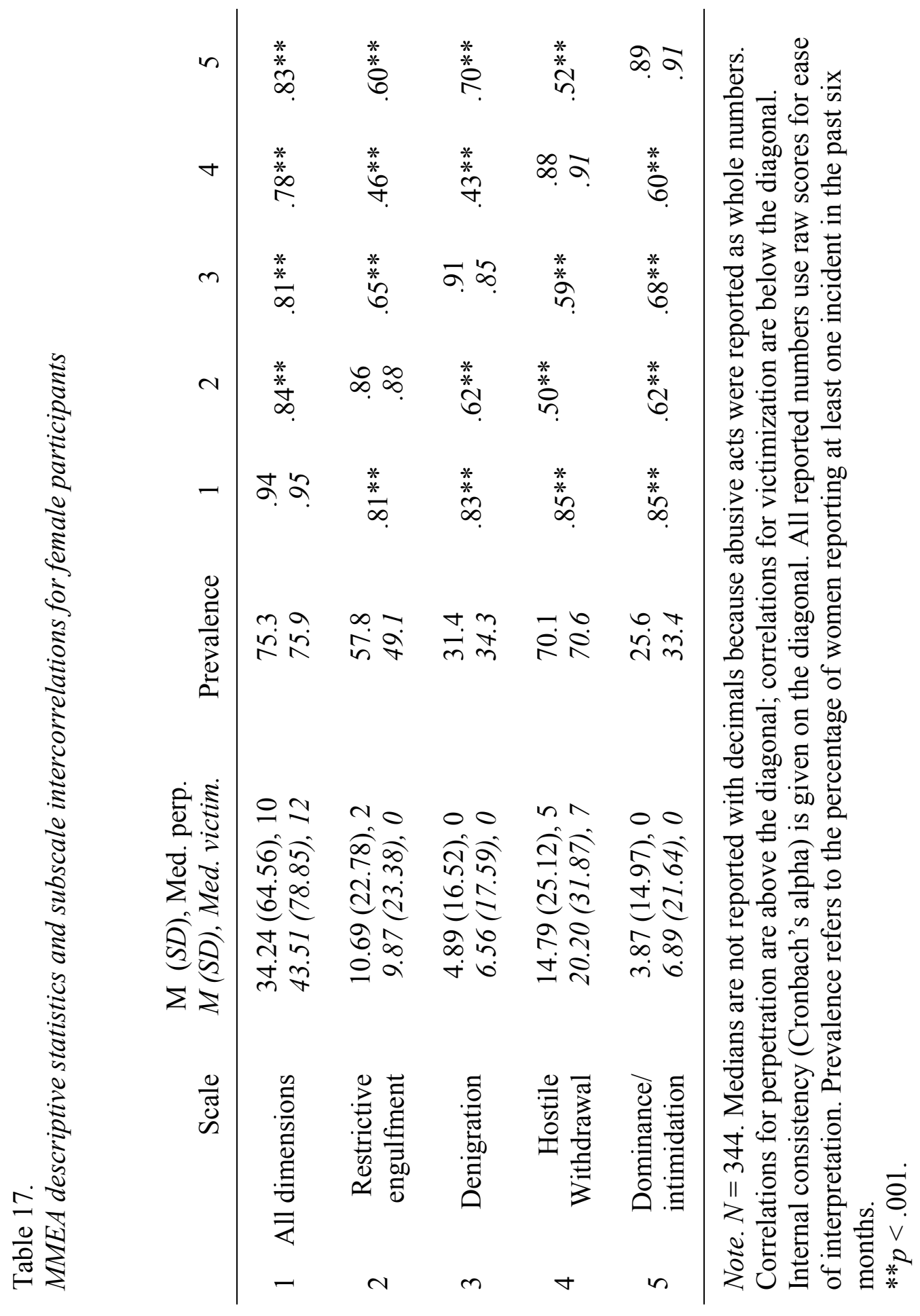




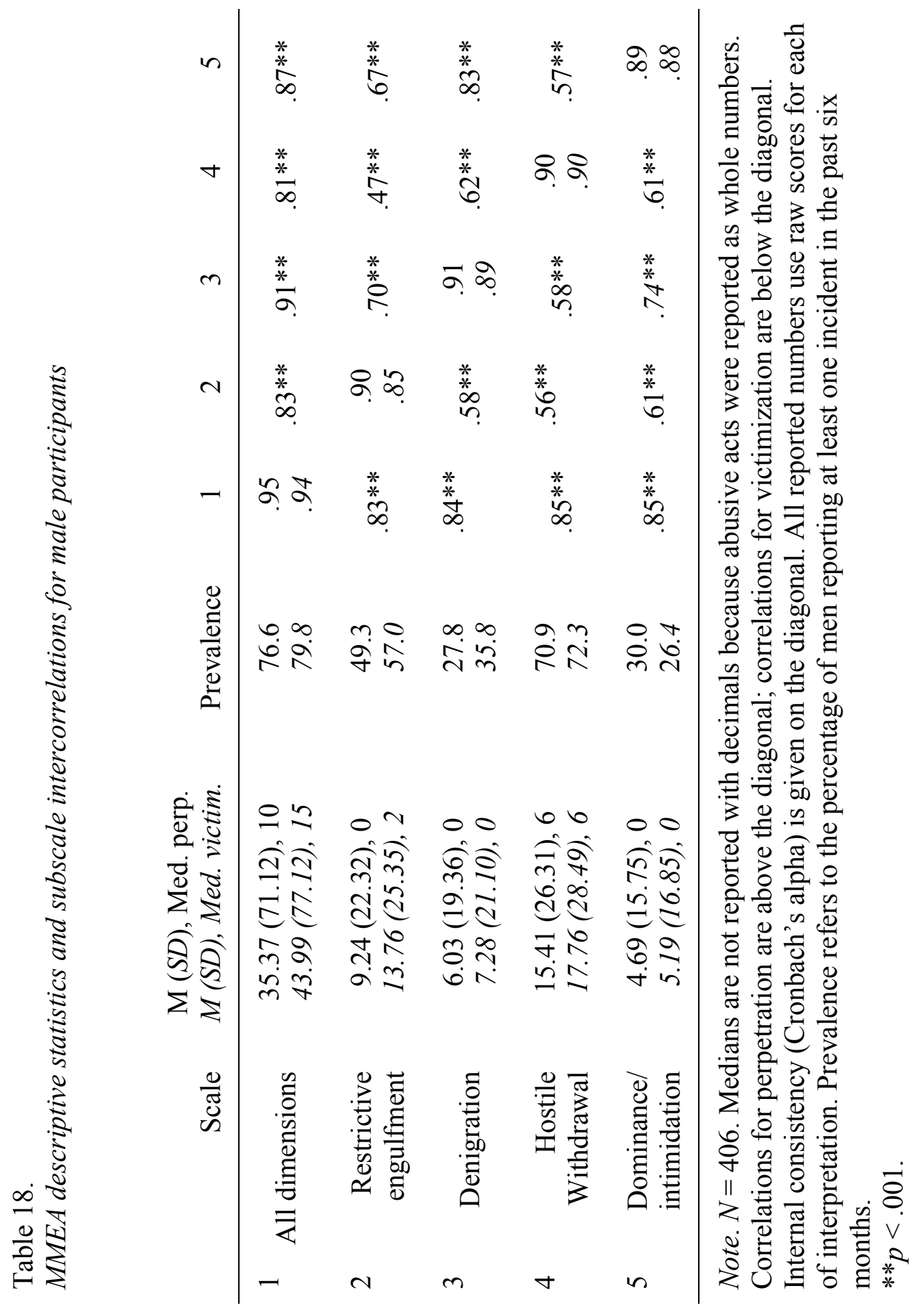


Table 19.

Hypothesized CFA: Feminine partner-ideals and-perceptions factor loadings

\begin{tabular}{|c|c|c|c|c|c|c|c|c|c|}
\hline \multirow[b]{2}{*}{ Factor } & \multirow[b]{2}{*}{ Item \# } & \multicolumn{4}{|c|}{ Feminine ideals } & \multicolumn{4}{|c|}{ Feminine perceptions } \\
\hline & & Loading & SE & $p$ & $\begin{array}{c}\text { Std. } \\
\text { loading }\end{array}$ & Loading & SE & $p$ & $\begin{array}{c}\text { Std. } \\
\text { Loading }\end{array}$ \\
\hline \multirow{9}{*}{$\begin{array}{r}\text { Stereotypic } \\
\text { images and } \\
\text { activities }\end{array}$} & 19 & .72 & .06 & $<.001$ & .55 & .31 & .07 & $<.001$ & .26 \\
\hline & 23 & .59 & .06 & $<.001$ & .51 & .42 & .07 & $<.001$ & .34 \\
\hline & 27 & .83 & .06 & $<.001$ & .69 & .52 & .05 & $<.001$ & .52 \\
\hline & 28 & .84 & .05 & $<.001$ & .71 & .65 & .06 & $<.001$ & .56 \\
\hline & 29 & .59 & .06 & $<.001$ & .50 & .02 & .07 & .80 & .01 \\
\hline & 31 & .62 & .05 & $<.001$ & .59 & .18 & .05 & .01 & .16 \\
\hline & 41 & .63 & .05 & $<.001$ & .59 & .48 & .07 & $<.001$ & .38 \\
\hline & 43 & .63 & .05 & $<.001$ & .58 & .43 & .06 & $<.001$ & .39 \\
\hline & 45 & .55 & .05 & $<.001$ & .53 & .56 & .07 & $<.001$ & .47 \\
\hline \multirow{11}{*}{$\begin{array}{r}\text { Dependency/ } \\
\text { deference }\end{array}$} & 1 & .67 & .06 & $<.001$ & .57 & .63 & .06 & $<.001$ & .52 \\
\hline & 3 & .33 & .05 & $<.001$ & .34 & .18 & .08 & .03 & .12 \\
\hline & 4 & .61 & .05 & $<.001$ & .58 & .58 & .08 & $<.001$ & .40 \\
\hline & 6 & .83 & .05 & $<.001$ & .74 & .65 & .06 & $<.001$ & .58 \\
\hline & 8 & .51 & .05 & $<.001$ & .55 & .41 & .07 & $<.001$ & .62 \\
\hline & 9 & .72 & .05 & $<.001$ & .72 & .65 & .05 & $<.001$ & .61 \\
\hline & 10 & .52 & .06 & $<.001$ & .46 & .54 & .06 & $<.001$ & .46 \\
\hline & 11 & .87 & .06 & $<.001$ & .71 & .43 & .06 & $<.001$ & .68 \\
\hline & 13 & .68 & .05 & $<.001$ & .65 & .50 & .06 & $<.001$ & .43 \\
\hline & 16 & .91 & .05 & $<.001$ & .79 & .71 & .06 & $<.001$ & .56 \\
\hline & 18 & .73 & .05 & $<.001$ & .67 & .57 & .06 & $<.001$ & .51 \\
\hline \multirow{9}{*}{ Purity } & 5 & .84 & .07 & $<.001$ & .60 & .49 & .06 & $<.001$ & .40 \\
\hline & 12 & 1.00 & .06 & $<.001$ & .77 & .97 & .06 & $<.001$ & .72 \\
\hline & 15 & 1.05 & .06 & $<.001$ & .77 & .75 & .07 & $<.001$ & .55 \\
\hline & 22 & .86 & .07 & $<.001$ & .61 & .87 & .07 & $<.001$ & .60 \\
\hline & 25 & 1.07 & .06 & $<.001$ & .82 & .87 & .07 & $<.001$ & .64 \\
\hline & 26 & .97 & .05 & $<.001$ & .82 & .90 & .06 & $<.001$ & .67 \\
\hline & 30 & 1.01 & .05 & $<.001$ & .83 & 1.02 & .06 & $<.001$ & .75 \\
\hline & 32 & .72 & .07 & $<.001$ & .54 & .30 & .08 & $<.001$ & .21 \\
\hline & 35 & .75 & .05 & $<.001$ & .65 & .62 & .06 & $<.001$ & .54 \\
\hline \multirow{7}{*}{ Caretaking } & 2 & .52 & .04 & $<.001$ & .64 & .69 & .05 & $<.001$ & .70 \\
\hline & 7 & .55 & .04 & $<.001$ & .71 & .61 & .04 & $<.001$ & .69 \\
\hline & 14 & .62 & .05 & $<.001$ & .61 & .68 & .05 & $<.001$ & .65 \\
\hline & 17 & .62 & .06 & $<.001$ & .53 & .58 & .06 & $<.001$ & .45 \\
\hline & 21 & .61 & .04 & $<.001$ & .71 & .67 & .05 & $<.001$ & .67 \\
\hline & 24 & .44 & .04 & $<.001$ & .54 & .53 & .05 & $<.001$ & .53 \\
\hline & 38 & .51 & .05 & $<.001$ & .52 & .64 & .06 & $<.001$ & .56 \\
\hline \multirow{9}{*}{ Emotionality } & 20 & .63 & .05 & $<.001$ & .60 & .49 & .07 & $<.001$ & .41 \\
\hline & 33 & .64 & .05 & $<.001$ & .61 & .68 & .06 & $<.001$ & .62 \\
\hline & 34 & .28 & .05 & $<.001$ & .32 & .36 & .06 & $<.001$ & .35 \\
\hline & 36 & .71 & .05 & $<.001$ & .64 & .73 & .06 & $<.001$ & .60 \\
\hline & 37 & .70 & .05 & $<.001$ & .66 & .62 & .06 & $<.001$ & .52 \\
\hline & 39 & .62 & .05 & $<.001$ & .63 & .85 & .06 & $<.001$ & .73 \\
\hline & 40 & .33 & .06 & $<.001$ & .31 & .24 & .06 & $<.001$ & .23 \\
\hline & 42 & .50 & .05 & $<.001$ & .52 & .18 & .07 & .01 & .15 \\
\hline & 44 & .55 & .05 & $<.001$ & .58 & .38 & .06 & $<.001$ & .34 \\
\hline
\end{tabular}


Table 20.

Hypothesized CFA: Feminine partner-ideals and-perceptions latent factor correlations

\begin{tabular}{llrrrrr} 
& & 1 & 2 & 3 & 4 & 5 \\
\hline 1 & Stereotypic image and activities & -- & $.79 * *$ & $.62^{* *}$ & $.56^{* *}$ & $.77^{* *}$ \\
2 & Dependence/deference & $.68^{* *}$ & -- & $.66^{* *}$ & $.24 * *$ & $.78^{* *}$ \\
3 & Purity & $.63^{* *}$ & $.47 * *$ & -- & $.31 * *$ & $.63 * *$ \\
4 & Caretaking & $.41^{* *}$ & $.19^{*}$ & $.25 * *$ & -- & $.43 * *$ \\
5 & Emotionality & $.44^{* *}$ & $.63^{* *}$ & $.30^{* *}$ & $.19^{*}$ & -- \\
\hline
\end{tabular}

Note. Correlations for partner-ideals $(N=406)$ are above the diagonal; correlations for partner-perceptions $(N=406)$ are below the diagonal.

$* p<.05$.

$* * p<.001$. 
Table 21.

Comparison of alternative feminine partner-ideals and-perceptions models

\begin{tabular}{|c|c|c|c|c|c|c|c|c|}
\hline Model & $\chi^{2}$ & df & CFI & RMSEA & $\begin{array}{c}\chi^{2} \\
\text { difference }\end{array}$ & $\mathrm{df}$ & $\mathrm{p}$ & $w$ \\
\hline \multicolumn{9}{|c|}{ Feminine ideals } \\
\hline Hypothesized & 3001.93 & 935 & .75 & .07 & -- & -- & -- & -- \\
\hline Single-factor & 4359.31 & 945 & .59 & .09 & 1357.38 & 10 & $<.001$ & .58 \\
\hline $\begin{array}{r}\text { Second-order } \\
\text { factor }\end{array}$ & 3054.82 & 940 & .75 & .08 & 52.89 & 5 & $<.001$ & .16 \\
\hline Six-factor & 2011.51 & 890 & .87 & .06 & -990.42 & 45 & $<.001$ & .23 \\
\hline \multicolumn{9}{|c|}{ Feminine perceptions } \\
\hline Hypothesized & 3046.01 & 935 & .59 & .08 & -- & -- & -- & -- \\
\hline Single-factor & 4149.03 & 945 & .37 & .09 & 1103.02 & 10 & $<.001$ & .52 \\
\hline $\begin{array}{r}\text { Second-order } \\
\text { factor }\end{array}$ & 3076.53 & 940 & .58 & .08 & 30.52 & 5 & $<.001$ & .12 \\
\hline Six-factor & 2300.28 & 890 & .73 & .06 & -745.73 & 45 & $<.001$ & .20 \\
\hline
\end{tabular}

Note. All $\chi^{2}$ difference tests compare the given model to the original, hypothesized model. 
Table 22.

Hypothesized CFA: Masculine partner-ideals and-perceptions factor loadings

\begin{tabular}{|c|c|c|c|c|c|c|c|c|c|}
\hline \multirow[b]{2}{*}{ Factor } & \multicolumn{5}{|c|}{ Masculine ideals } & \multicolumn{4}{|c|}{ Masculine perceptions } \\
\hline & Item \# & Loading & SE & $p$ & $\begin{array}{c}\text { Std. } \\
\text { Loading }\end{array}$ & Loading & SE & $p$ & $\begin{array}{c}\text { Std. } \\
\text { Loading }\end{array}$ \\
\hline \multirow{3}{*}{$\begin{array}{l}\text { Avoidance of } \\
\text { femininity }\end{array}$} & 4 & 1.55 & .09 & $<.001$ & .82 & 1.11 & .13 & $<.001$ & .52 \\
\hline & 8 & 1.39 & .09 & $<.001$ & .78 & 1.19 & .10 & $<.001$ & .81 \\
\hline & 10 & .98 & .10 & $<.001$ & .54 & .62 & .08 & $<.001$ & .47 \\
\hline Negativity & 1 & 1.80 & .08 & $<.001$ & .91 & 1.89 & .10 & $<.001$ & .87 \\
\hline \multirow{2}{*}{$\begin{array}{r}\text { toward sexual } \\
\text { minorities }\end{array}$} & 5 & 1.35 & .08 & $<.001$ & .80 & 1.46 & .09 & $<.001$ & .77 \\
\hline & 13 & 1.90 & .08 & $<.001$ & .97 & 2.03 & .10 & $<.001$ & .90 \\
\hline \multirow{3}{*}{ Self-reliance } & 6 & 1.08 & .05 & $<.001$ & .93 & 1.51 & .07 & $<.001$ & .93 \\
\hline & 7 & 1.09 & .05 & $<.001$ & .98 & 1.66 & .07 & $<.001$ & .98 \\
\hline & 14 & 1.01 & .07 & $<.001$ & .71 & 1.43 & .10 & $<.001$ & .70 \\
\hline \multirow{3}{*}{ Toughness } & 19 & .78 & .09 & $<.001$ & .50 & 1.11 & .10 & $<.001$ & .64 \\
\hline & $20 \mathrm{a}$ & 1.53 & .09 & $<.001$ & .82 & 1.29 & .11 & $<.001$ & .68 \\
\hline & $20 \mathrm{~b}$ & 1.30 & .08 & $<.001$ & .76 & 1.15 & .11 & $<.001$ & .60 \\
\hline \multirow{3}{*}{ Dominance } & 2 & .88 & .09 & $<.001$ & .52 & 1.05 & .11 & $<.001$ & .54 \\
\hline & 3 & 1.42 & .07 & $<.001$ & .82 & 1.48 & .10 & $<.001$ & .78 \\
\hline & 12 & 1.43 & .08 & $<.001$ & .88 & 1.47 & .11 & $<.001$ & .72 \\
\hline \multirow{3}{*}{$\begin{array}{r}\text { Importance of } \\
\text { sex }\end{array}$} & 9 & 1.46 & .08 & $<.001$ & .83 & 1.67 & .09 & $<.001$ & .86 \\
\hline & 11 & 1.36 & .08 & $<.001$ & .78 & 1.50 & .08 & $<.001$ & .82 \\
\hline & 18 & 1.66 & .07 & $<.001$ & .95 & 1.83 & .08 & $<.001$ & .94 \\
\hline \multirow{3}{*}{$\begin{array}{r}\text { Restrictive } \\
\text { emotionality }\end{array}$} & 15 & 1.22 & .07 & $<.001$ & .86 & 1.62 & .10 & $<.001$ & .84 \\
\hline & 16 & 1.15 & .08 & $<.001$ & .74 & 1.58 & .11 & $<.001$ & .78 \\
\hline & 21 & 1.01 & .09 & $<.001$ & .60 & 1.26 & .10 & $<.001$ & .64 \\
\hline
\end{tabular}

Note. Item 17 in the Toughness factor was replaced by an alternate adaptation of item 20 based on factor loadings in the second pilot study. 
Table 23.

Hypothesized CFA: Masculine partner-ideals and-perceptions latent factor correlations

\begin{tabular}{rrccccccc} 
& & 1 & 2 & 3 & 4 & 5 & 6 & 7 \\
\hline 1 & $\begin{array}{r}\text { Avoidance of } \\
\text { femininity }\end{array}$ & -- & $.53^{* *}$ & $.51^{* *}$ & $.74^{* *}$ & $.69^{* *}$ & $.45^{* *}$ & $.43^{* *}$ \\
& $\begin{array}{r}\text { Negativity toward } \\
\text { sexual minorities }\end{array}$ & $.29^{* *}$ & -- & $.20^{* *}$ & $.47^{* *}$ & $.38^{* *}$ & $.25^{* *}$ & $.42^{* *}$ \\
3 & $\begin{array}{r}\text { Self-reliance through } \\
\text { mechanical skills }\end{array}$ & $.32^{* *}$ & $.19^{* *}$ & -- & $.68^{* *}$ & $.51^{* *}$ & $.42^{* *}$ & .10 \\
4 & Dominance & $.41^{* *}$ & $.40^{* *}$ & $.41^{* *}$ & -- & $.74^{* *}$ & $.55^{* *}$ & $.24 * *$ \\
5 & Toughness & $.45^{* *}$ & $.35^{* *}$ & $.48^{* *}$ & $.63^{* *}$ & -- & $.60^{* *}$ & $.50^{* *}$ \\
6 & Importance of sex & $.29^{* *}$ & $.23^{* *}$ & $.27^{* *}$ & $.36^{* *}$ & $.61^{* *}$ & -- & $.31^{* *}$ \\
7 & $\begin{array}{r}\text { Restrictive } \\
\text { emotionality }\end{array}$ & $.32^{* *}$ & $.34^{* *}$ & $.15^{*}$ & $.25^{* *}$ & $.30^{* *}$ & $.18^{*}$ & -- \\
\hline
\end{tabular}

Note. Correlations for partner-ideals $(N=344)$ are above the diagonal; correlations for partner-perceptions $(N=344)$ are below the diagonal.

$* p<.05$.

$* * p<.001$. 
Table 24.

Comparison of alternative masculine partner-ideals and-perceptions models

\begin{tabular}{|c|c|c|c|c|c|c|c|c|}
\hline Model & $\chi^{2}$ & df & CFI & RMSEA & $\begin{array}{c}\chi^{2} \\
\text { difference }\end{array}$ & df & $p$ & $w$ \\
\hline \multicolumn{9}{|c|}{ Masculine ideals } \\
\hline Hypothesized & 419.05 & 168 & .94 & .07 & -- & -- & -- & -- \\
\hline Single-factor & 2434.59 & 189 & .50 & .19 & 2015.54 & 21 & $<.001$ & .58 \\
\hline $\begin{array}{r}\text { Second-order } \\
\text { factor }\end{array}$ & 530.78 & 182 & .92 & .08 & 111.73 & 14 & $<.001$ & .16 \\
\hline Six-factor & 284.97 & 147 & .97 & .05 & -134.08 & 21 & $<.001$ & .23 \\
\hline \multicolumn{9}{|c|}{ Masculine perceptions } \\
\hline Hypothesized & 393.08 & 168 & .94 & .06 & -- & -- & -- & -- \\
\hline Single-factor & 2376.62 & 189 & .38 & .18 & 1983.54 & 21 & $<.001$ & .52 \\
\hline $\begin{array}{l}\text { Second-order } \\
\text { factor }\end{array}$ & 423.59 & 182 & .93 & .06 & 30.51 & 14 & .01 & .12 \\
\hline Six-factor & 236.31 & 147 & .98 & .04 & -156.47 & 21 & $<.001$ & .20 \\
\hline
\end{tabular}

Note. All $\chi^{2}$ difference tests compare the given model to the original, hypothesized model. 


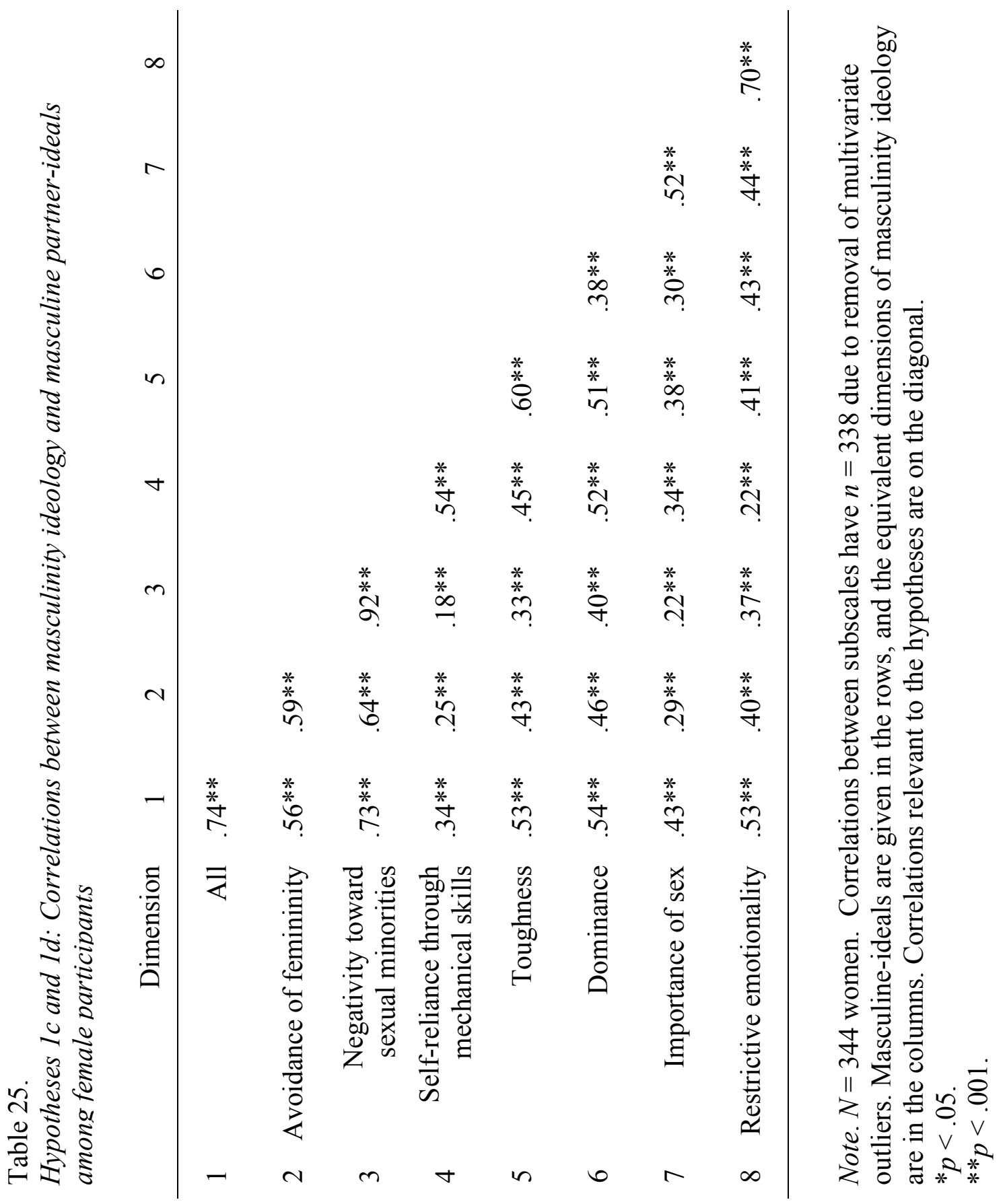


Table 26.

Hypothesis 2a: Mediation models testing effect of women's masculine partner-ideal discrepancies on physical intimate partner violence perpetration via relationship satisfaction

\begin{tabular}{rcccccc}
\multicolumn{7}{c}{ Physical assault } \\
& $b$ & $S E$ & $Z$ & $p$ & $\begin{array}{c}95 \% \text { C.I. } \\
\text { (Lower) }\end{array}$ & $\begin{array}{c}95 \% \text { C.I. } \\
\text { (Upper) }\end{array}$ \\
\hline Constant & -.57 & .65 & -.87 & .38 & -1.85 & .71 \\
$\mathrm{X} \rightarrow \mathrm{M}(\mathrm{a})$ & -2.16 & .17 & -12.54 & $<.001$ & -2.50 & -1.83 \\
$\mathrm{M} \rightarrow \mathrm{Y}(\mathrm{b})$ & -.18 & .14 & -1.34 & .18 & -.45 & .08 \\
$\mathrm{X} \rightarrow \mathrm{Y}(\mathrm{c})$ & .39 & .58 & .69 & .49 & -.71 & 1.48 \\
$\mathrm{X} \rightarrow \mathrm{M} \rightarrow \mathrm{Y}\left(\mathrm{c}^{\prime}\right)$ & .39 & .30 & -- & -- & -.18 & .98 \\
\hline Nagelkerke $R^{2}$ & .03 & & & & \\
$-2 L L$ & 279.68 & & & & & \\
$p$ & .09 & & & & & \\
\hline
\end{tabular}

\begin{tabular}{|c|c|c|c|c|c|c|}
\hline & \multicolumn{4}{|c|}{ fury } & \multirow{2}{*}{$\begin{array}{l}\text { 95\% C.I. } \\
\text { (Lower) }\end{array}$} & \multirow{2}{*}{$\begin{array}{r}95 \% \text { C.I. } \\
\text { (Upper) }\end{array}$} \\
\hline & $b$ & $S E$ & $Z$ & $p$ & & \\
\hline Constant & -.96 & .82 & -1.18 & .24 & -2.56 & .64 \\
\hline$X \rightarrow M(a)$ & -2.16 & .17 & -12.54 & $<.001$ & -2.50 & -1.83 \\
\hline $\mathrm{M} \rightarrow \mathrm{Y}(\mathrm{b})$ & -.27 & .18 & -1.52 & .13 & -.62 & .08 \\
\hline $\mathrm{X} \rightarrow \mathrm{Y}(\mathrm{c})$ & .51 & .78 & .65 & .51 & -1.03 & 2.05 \\
\hline $\mathrm{X} \rightarrow \mathrm{M} \rightarrow \mathrm{Y}\left(\mathrm{c}^{\prime}\right)$ & .59 & .38 & -- & -- & -.17 & 1.36 \\
\hline Nagelkerke $R^{2}$ & .04 & & & & & \\
\hline$-2 L L$ & 157.47 & & & & & \\
\hline$p$ & .05 & & & & & \\
\hline
\end{tabular}

Note. $N=342$ women. $\mathrm{X}=$ Masculine-ideal discrepancy, $\mathrm{M}=\mathrm{PRQC}$ score (mediator), $\mathrm{Y}$ $=$ CTS 2 perpetration score (dichotomized such that $0=$ no IPV and $1=$ IPV). PROCESS does not calculate total effects or measures of effect size for indirect effects when $\mathrm{Y}$ is dichotomous. 
Table 27.

Hypothesis 2b: Mediation models testing the effect of women's masculine partner-ideal discrepancies on emotional abuse perpetration via relationship satisfaction

\begin{tabular}{|c|c|c|c|c|c|c|}
\hline & $b$ & $S E$ & $t$ & $p$ & $\begin{array}{c}95 \% \text { C.I. } \\
\text { (Lower) }\end{array}$ & $\begin{array}{c}\text { 95\% C.I. } \\
\text { (Upper) }\end{array}$ \\
\hline Constant & 1.29 & .07 & 18.55 & $<.001$ & 1.15 & 1.42 \\
\hline$X \rightarrow M(a)$ & -2.16 & .17 & -12.54 & $<.001$ & -2.50 & -1.83 \\
\hline $\mathrm{M} \rightarrow \mathrm{Y}(\mathrm{b})$ & -.13 & .04 & -3.47 & $<.001$ & -.20 & -.06 \\
\hline $\mathrm{X} \rightarrow \mathrm{Y}(\mathrm{c})$ & .32 & .14 & 2.21 & .03 & .03 & .60 \\
\hline $\mathrm{X} \rightarrow \mathrm{M} \rightarrow \mathrm{Y}\left(\mathrm{c}^{\prime}\right)$ & .28 & .08 & -- & -- & .12 & .45 \\
\hline Total $\mathrm{X} \rightarrow \mathrm{Y}$ & .60 & .12 & 4.95 & $<.001$ & .36 & .84 \\
\hline \multicolumn{7}{|l|}{$R^{2}$} \\
\hline$F(d f)$ & \multicolumn{6}{|c|}{$24.54(1,340)$} \\
\hline$p$ & \multicolumn{6}{|c|}{$<.001$} \\
\hline
\end{tabular}

Note. $N=342$ women. $\mathrm{X}=$ Masculine-ideal discrepancy, $\mathrm{M}=\mathrm{PRQC}$ score (mediator), $\mathrm{Y}$ $=$ MMEA perpetration score (log transformed). 
Table 28.

Hypotheses $3 a$ and 3b: Correlations between men's perceived gender role conformity and IPV perpetration

\begin{tabular}{|c|c|c|c|c|c|c|}
\hline \multirow[b]{3}{*}{ Male role norm } & \multicolumn{4}{|c|}{ CTS2 } & & \\
\hline & \multicolumn{2}{|c|}{ Physical Assault } & \multicolumn{2}{|c|}{ Injury } & \multicolumn{2}{|c|}{ MMEA } \\
\hline & $r$ & $1-\beta$ & $r$ & $1-\beta$ & $r$ & $1-\beta$ \\
\hline Total role conformity & .05 & .15 & .08 & .32 & $.15^{*}$ & .80 \\
\hline Avoidance of femininity & -.07 & .25 & -.08 & .32 & .03 & .09 \\
\hline $\begin{array}{r}\text { Negativity toward sexual } \\
\text { minorities }\end{array}$ & .08 & .32 & $.17^{*}$ & .89 & $.20 * *$ & .97 \\
\hline $\begin{array}{r}\text { Self-reliance through } \\
\text { mechanical skills }\end{array}$ & .06 & .20 & -.02 & .07 & .00 & .05 \\
\hline Toughness & .05 & .15 & .02 & .07 & .01 & .05 \\
\hline Dominance & -.03 & .09 & -.06 & .20 & -.03 & .09 \\
\hline Importance of sex & -.03 & .09 & .00 & .05 & -.01 & .05 \\
\hline Restrictive emotionality & $.12 *$ & .61 & $.11 *$ & .53 & $.40 * *$ & 1.00 \\
\hline
\end{tabular}

Note. $N=344$ women. CTS2 physical assault and injury scores were dichotomized.

MMEA scores were log-transformed. $1-\beta$ represents the achieved power of the analysis.

$* p<.05$.

$* * p<.001$. 
Table 29.

Post hoc analyses on IPV: Bivariate correlations between PRQC mean scores, masculine partner-ideal discrepancies, and IPV perpetration/victimization

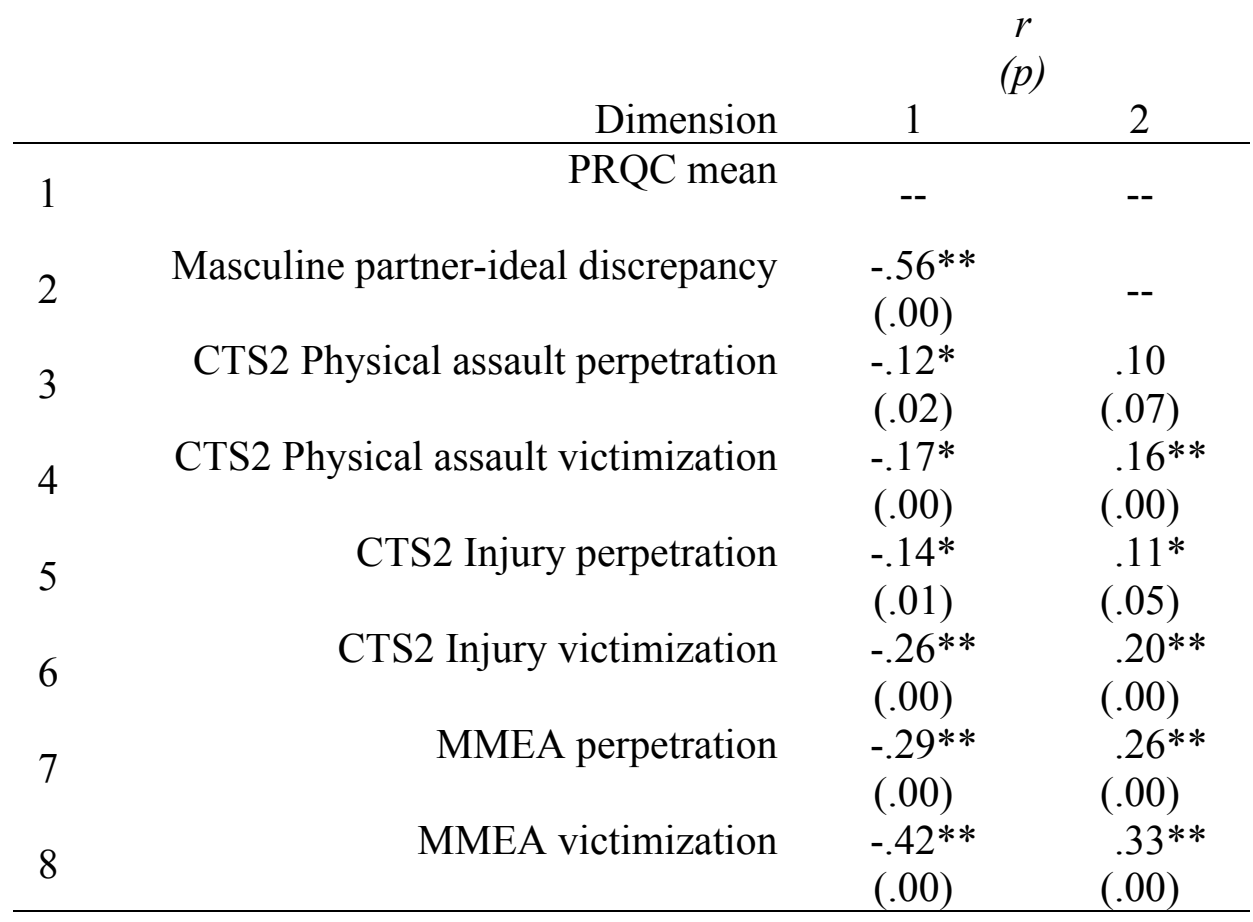

Note. $N=344$ women. CTS2 physical assault and injury scores were dichotomized. MMEA scores were log-transformed.

$* p<.05$.

$* * p<.001$. 
Table 30.

Summary of results of hypothesis testing

\begin{tabular}{|c|c|c|c|}
\hline \multicolumn{2}{|c|}{ Hypothesis } & Summary & Supported by results? \\
\hline \multirow[t]{4}{*}{1} & $\mathrm{a}$ & $\begin{array}{l}\text { Aggregate femininity ideology positively } \\
\text { correlates with aggregate feminine-ideals }\end{array}$ & $--*$ \\
\hline & $\mathrm{b}$ & $\begin{array}{l}\text { Each dimension of femininity ideology } \\
\text { positively correlates with the corresponding } \\
\text { feminine-ideals dimension }\end{array}$ & $--^{*}$ \\
\hline & $\mathrm{c}$ & $\begin{array}{l}\text { Aggregate masculinity ideology positively } \\
\text { correlates with aggregate masculine-ideals }\end{array}$ & Yes \\
\hline & d & $\begin{array}{l}\text { Each dimension of masculinity ideology } \\
\text { positively correlates with the corresponding } \\
\text { masculine-ideals dimension }\end{array}$ & Yes \\
\hline \multirow[t]{2}{*}{2} & $\mathrm{a}$ & $\begin{array}{l}\text { Gendered partner-ideal discrepancies positively } \\
\text { predict physical IPV perpetration (i.e., physical } \\
\text { assault and injury), mediated by relationship } \\
\text { satisfaction }\end{array}$ & No (in women*) \\
\hline & $\mathrm{b}$ & $\begin{array}{l}\text { Gendered partner-ideal discrepancies positively } \\
\text { predict emotional IPV perpetration, mediated } \\
\text { by relationship satisfaction }\end{array}$ & Yes (in women*) \\
\hline \multirow[t]{4}{*}{3} & $\mathrm{a}$ & $\begin{array}{l}\text { Aggregate masculine-perceptions positively } \\
\text { correlate with women's IPV victimization (both } \\
\text { physical and emotional) }\end{array}$ & Partially \\
\hline & $\mathrm{b}$ & $\begin{array}{l}\text { Each dimension of masculine-perceptions } \\
\text { positively correlates with women's IPV } \\
\text { victimization (both physical and emotional) }\end{array}$ & Partially \\
\hline & $\mathrm{c}$ & $\begin{array}{l}\text { Aggregate feminine-perceptions negatively } \\
\text { correlate with men's IPV victimization (both } \\
\text { physical and emotional) }\end{array}$ & $--^{*}$ \\
\hline & d & $\begin{array}{l}\text { Each dimension of feminine-perceptions } \\
\text { negatively correlates with men's IPV } \\
\text { victimization (both physical and emotional) }\end{array}$ & - - $^{*}$ \\
\hline
\end{tabular}

*These hypotheses could not be tested in men because of the measurement problems discovered in the feminine-ideals and-perceptions measures. 


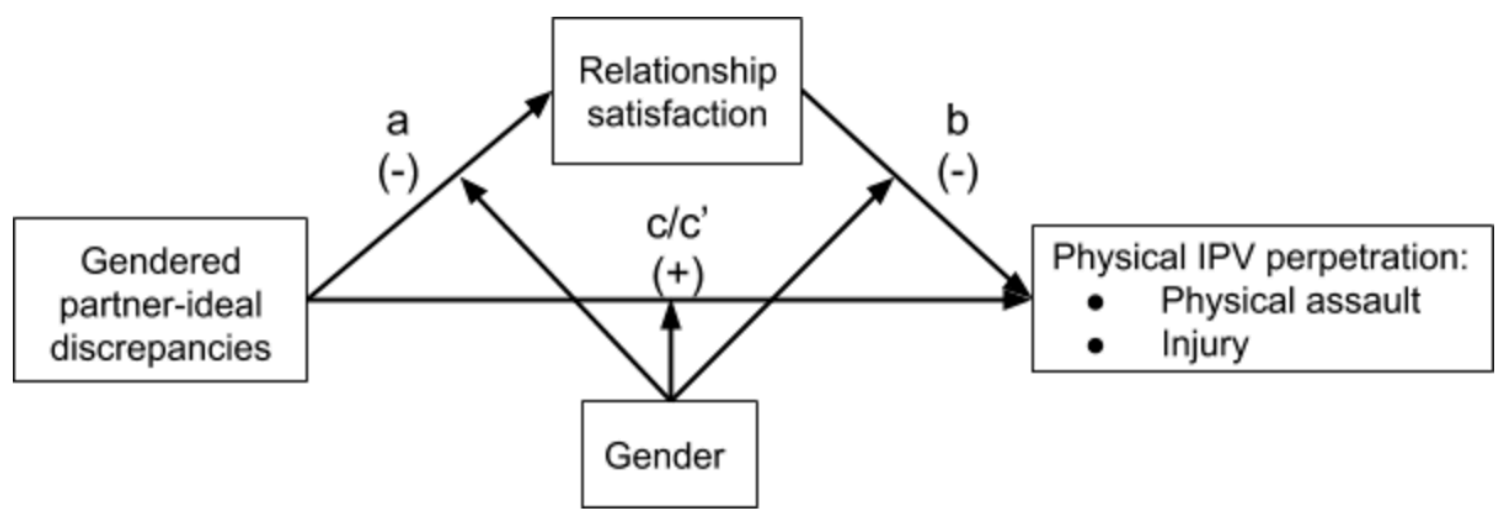

Figure 1. The proposed moderated mediation model for Hypothesis $2 \mathrm{a}$. 


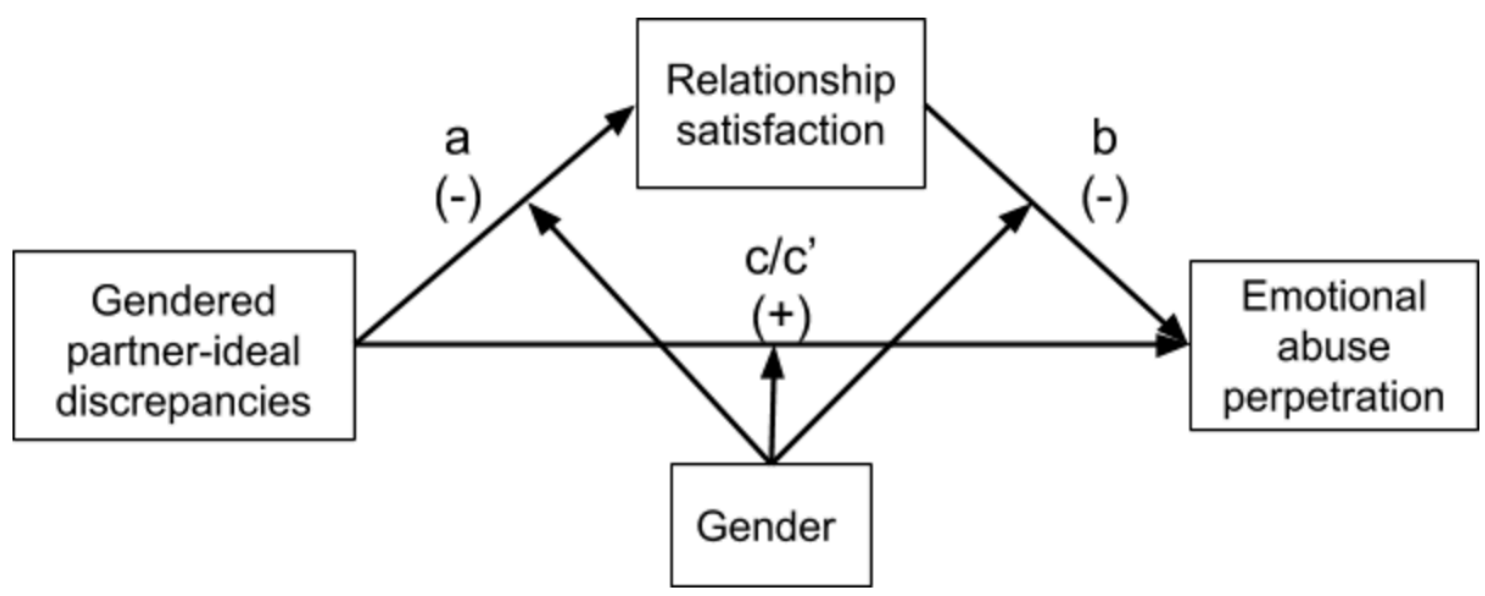

Figure 2. The proposed moderated mediation model for Hypothesis $2 \mathrm{~b}$. 


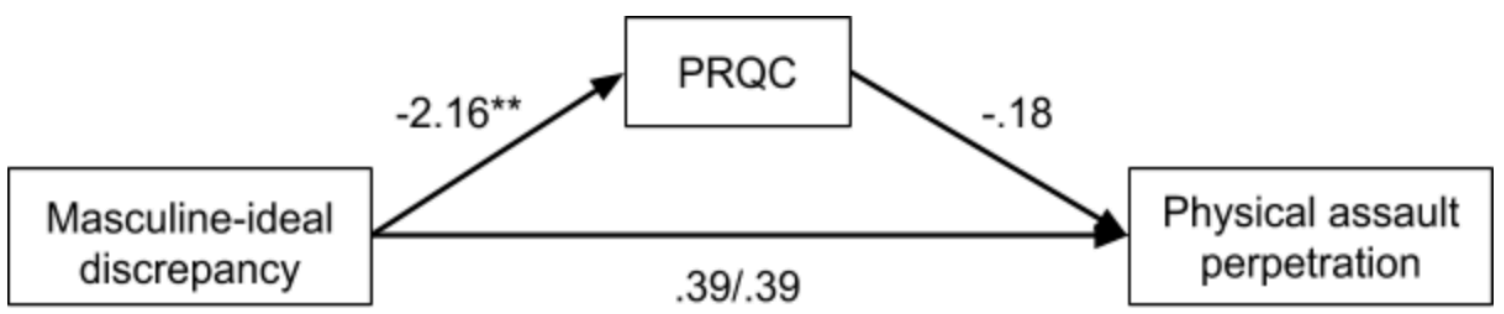

Figure 3. Hypothesis 2a: Results of the mediation model for women's physical assault perpetration 


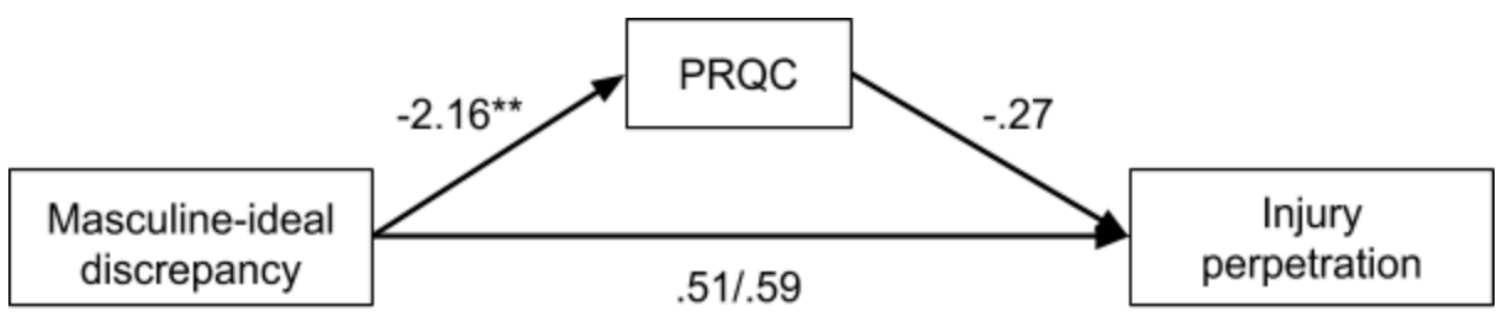

Figure 4. Hypothesis 2a: Results of the mediation model for women's injury perpetration 


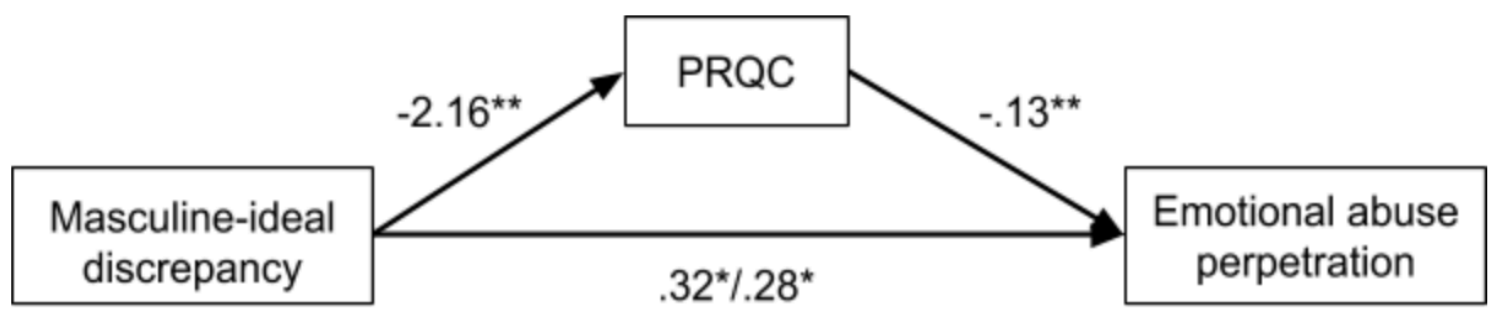

Figure 5. Hypothesis 2b: Results of the mediation model for women's emotional abuse perpetration 


\section{References}

Ackerman, J. M. (2015). Over-reporting intimate partner violence in Australian survey research. British Journal of Criminology, 56, 646-667. doi:10.1093/bjc/azv066

Ackerman, J., \& Field, L. (2011). The gender asymmetric effect of intimate partner violence on relationship satisfaction. Violence and Victims, 26, 1-22. doi:10.1891/10886-6708.26.6.1

Ali, P. A., Dhingra, K., \& McGarry, J. (2016). A literature review of intimate partner violence and its classifications. Aggression and Violent Behavior, 31, 16-25. doi:10.1016/j.avb.2016.06.008

Ali, P. A., \& Naylor, P. B. (2013). Intimate partner violence: A narrative review of the biological and psychological explanations for its causation. Aggression and Violent Behavior, 18, 373-382. doi:10.1016/j.avb.2013.01.003

Ali, P. A., \& Naylor, P. B. (2013). Intimate partner violence: A narrative review of the feminist, social and ecological explanations for its causation. Aggression and Violent Behavior, 18, 611-619. doi:10.1016/j.avb.2013.07.009

Amato, P. R., \& Booth, A. (1995). Changes in gender role attitudes and perceived marital quality. American Sociological Review, 60, 58-66.

Anderson, K. L. (1997). Gender, status, and domestic violence: An integration of feminist and family violence approaches. Journal of Marriage and Family, 59, 655-669.

Anderson, K. L. (2002). Perpetrator or victim? Relationships between intimate partner violence and well-being. Journal of Marriage and Family, 64, 851-863.

Archer, J. (2000). Sex differences in aggression between heterosexual partners: A metaanalytic review. Psychological Bulletin, 126, 651-680. doi:10.1037/00332909.126.5.651

Archer, J. (2006). Cross-cultural differences in physical aggression between partners: A social-role analysis. Personality and Social Psychology Review, 10, 133-153.

Atkinson, M. P., Greenstein, T. N., \& Lang, M. M. (2005). For women, breadwinning can be dangerous: Gendered resource theory and wife abuse. Journal of Marriage and Family, 67, 1137-1148.

Aube, J., \& Koestner, R. (1995). Gender characteristics and relationship adjustment: Another look at similarity-complementarity hypotheses. Journal of Personality, 63, 879-904.

Avivi, Y. E., Laurenceau, J., \& Carver, C. S. (2009). Linking relationship quality to perceived mutuality of relationship goals and perceived goal progress. Journal of Social and Clinical Psychology, 28, 137-164.

Aylor, B., \& Dainton, M. (2004). Biological sex and psychological gender as predictors of routine and strategic relational maintenance. Sex Roles, 50, 689-697.

Backus, F. R., Mahalik, J. R., (2011). The masculinity of Mr. Right: Feminist identity and heterosexual women's ideal romantic partners. Psychology of Women Quarterly, 35, 318-326.

Bair-Merritt, M. H., Crowne, S. S., Thompson, D. A., Sibinga, E., Trent, M., \& Campbell, J. (2010). Why do women use intimate partner violence? A systematic review of women's motivations. Trauma, Violence, \& Abuse, 11, 178-189. 
doi: $10.1177 / 1534838010379003$

Baker, C. R., \& Stith, S. M. (2008). Factors predicting dating violence perpetration among male and female college students. Journal of Aggression, Maltreatment, \& Trauma, 17, 227-244.

Baron, R. M., \& Kenny, D. A. (1986). The moderator-mediator variable distinction in social psychological research: Conceptual, strategic, and statistical considerations. Journal of Personality and Social Psychology, 51, 1773-1182.

Bates, E. A. (2011). The relationship of men's and women's partner violence to personality and psychopathology. (Unpublished doctoral dissertation). University of Central Lancashire, UK.

Bentler, P. M., \& Chou, C. (1987). Practical issues in structural modeling. Sociological Methods \& Research, 16, 78-117.

Berinsky, A. J., Huber, G. A., \& Lenz, G. S. (2012). Evaluating online labor markets for experimental research: Amazon.com's Mechanical Turk. Political Analysis, 20, 351-368. doi:10.1093/pan/mpr057

Berke, D. S., Sloan, C. A., Parrott, D., \& Zeichner, A. (2012). Effects of female gender role and gender norm conformity on aggression in men: Does positive masculinity reduce the risk? Psychology of Men \& Masculinity, 13, 367-378. doi:10.1037/a0026525

Bogaert, A. F., Fawcett, C. C., \& Jamieson, L. K. (2009). Attractiveness, body size, masculine sex roles and 2D:4D ratios in men. Personality and Individual Differences, 47, 273-278. doi:10.1016/j.paid.2009.03.011

Bowen, E. (2011). Theories of intimate partner violence. In The Rehabilitation of Partner-Violent Men. Chichester, UK: John Wiley \& Sons, Ltd. doi:10.1002/9780470978603.ch3

Breiding, M. J. (2004). Observed hostility and dominance as mediators of the relationship between husbands' gender role conflict and wives' outcomes. Journal of Counseling Psychology, 51, 429-436. doi:10.1037/0022-0167.51.4.429

Breiding, M. J., Smith, S. G., Basile, K. C., Walters, M. L., Chen, J., \& Merrick, M. T. (2014). Prevalence and characteristics of sexual violence, stalking, and intimate partner violence victimization - National Intimate Partner and Sexual Violence Survey, United States, 2011. Retrieved from www.cdc.gov/mmwr/preview/mmwrhtml/ss6308a1.htm?s_cid=ss6308a1_e

Breiding, M. J., Windle, C. R., \& Smith, D. A. (2008). Interspousal criticism: A behavioral mediator between husbands' gender role conflict and wives' adjustment. Sex Roles, 59, 880-888. doi:10.1007/s11199-008-9491-6

Brock, R. L., Barry, R. A., Lawrence, E., Rolffs, J., Cerretani, J., \& Zarling, A. (2015). Online administration of questionnaires assessing psychological, physical, and sexual aggression: Establishing psychometric equivalence.

Brown, T. A. (2006) Confirmatory factor analysis for applied research. New York, NY: The Guilford Press.

Browning, J., \& Dutton, D. (1986). Assessment of wife assault with the Conflict Tactics Scale: Using couple data to quantify the differential reporting effect. Journal of Marriage and Family, 48, 375-379. 
Bryant, A. N. (2003). Changes in attitudes toward women's roles: Predicting gender-role traditionalism among college students. Sex Roles, 48, 131-142. doi:10.1023/A:102245120592

Burn, S. M., \& Ward, A. Z. (2005). Men's conformity to traditional masculinity and relationship satisfaction. Psychology of Men \& Masculinity, 6, 254-263, doi:10.1037/1524-9220.6.4.254

Busby, D. M., Christensen, C., Crane, D. R., \& Larson, J. H. (1995). A revision of the Dyadic Adjustment Scale for use with distressed and nondistressed couples: Construct hierarchy and multidimensional scales. Journal of Marital and Family Therapy, 21, 289-308.

Buss, D. M., (1989). Sex differences in human mate preferences: Evolutionary hypotheses tested in 37 cultures. Behavioral and Brain Sciences, 12, 1-49.

Buss, D. M., Shackelford, T. K., Kirkpatrick, L. A., \& Larsen, R. J. (2001). A half century of mate preferences: The cultural evolution of values. Journal of Marriage and Family, 63, 491-503.

Cafri, G., van den Berg, P., \& Brannick, M. T. (2010). What have the difference scores not been telling us? A critique of the use of self-ideal discrepancy in the assessment of body image and evaluation of an alternative data-analytic framework. Assessment, 17, 361-376. doi:10.1177/1073191109357028.

Campbell, L., Overall, N. C., Rubin, H., \& Lackenbauer, S. D. (2016). Inferring a partner's ideal discrepancies: Accuracy, projection, and the communicative role of interpersonal behavior. Journal of Personality and Social Psychology, 105, 217-233. doi:10.1037/10022009

Campbell, L., Simpson, J. A., Kashy, D. A., \& Fletcher, G. J. O. (2001). Ideal standards, the self, and flexibility of ideals in close relationships. Personality and Social Psychology Bulletin, 27, 447-462.

Campbell, J. L., \& Snow, B. M. (1992). Gender role conflict and family environment as predictors of men's marital satisfaction. Journal of Family Psychology, 6, 84-87.

Capaldi, D. M., Knoble, N. B., Shortt, J. W., \& Kim, H. K. (2012). A systematic review of risk factors for intimate partner violence. Partner Abuse, 3, 231-280. doi:10.1891/1946-6560.3.2.231

Casad, B. J., Salazar, M. M., \& Macina, V. (2014). The real versus the ideal: Predicting relationship satisfaction and well-being from endorsement of marriage myths and benevolent sexism. Psychology of Women Quarterly, 39, 1-11. doi: $10.1177 / 0361684314528304$

Centers for Disease Control and Prevention. (2016). Intimate partner violence. Retrieved from www.cdc.gov/violenceprevention/intimatepartnerviolence/

Cercone, J. J., Beach, S. R. H., \& Arias, I. (2005). Gender symmetry in dating intimate partner violence: Does similar behavior imply similar constructs? Violence and Victims, 20, 207-218.

Chen, F. F. (2007). Sensitivity of goodness of fit indexes to lack of measurement invariance. Structural Equation Modeling, 14, 464-504.

Cheung, A. K., \& Choi, S. Y. (2016). Non-traditional wives with traditional husbands: Gender ideology and husband-to-wife physical violence in Chinese society. 
Violence Against Women, 22, 1704-1724. doi:10.1177/1077801216632615

Cialdini, R. B., \& Trost, M. R. (1998). Social influence: Social norms, conformity, and compliance. In D. T. Gilbert, S. T. Fiske, \& L. Gardner (Eds.), The handbook of social psychology (151-192). New York, NY: McGraw-Hill.

Cohen, J. (1992). A power primer. Psychological Bulletin, 112, 155-159.

Cohn, A., \& Zeichner, A. (2006). Effects of masculine identity and gender role stress on aggression in men. Psychology of Men \& Masculinity, 7, 179-190. doi:10.1037/1524-9220.7.4.179

Cortina, J. M. (1993). What is coefficient alpha? An examination of theory and application. Journal of Applied Psychology, 78, 98-104.

Coughlin, P., \& Wade, J. C. (2012). Masculinity ideology, income disparity, and romantic relationship quality among men with higher earning female partners. Sex Roles, 67, 311-322. doi:10.1007/s11199-012-0187-6

Davis, S. N., \& Greenstein, T. N. (2009). Gender ideology: Components, predictors, and consequences. Annual Review of Sociology, 35, 87-105. doi:10.1146/annurev-soc070308-115920

Deaux, K., \& Major, B. (1987). Putting gender into context: An interactive model of gender-related behavior. Psychological Review, 94, 369-389.

Delacollette, N., Dumont, M., Sarlet, M., \& Dardenne, B. (2013). Benevolent sexism, men's advantages and the prescription of warmth to women. Sex Roles, 68, 296310. doi:10.1007/s11199-012-0232-5

DeMaris, A., Benson, M. L., Fox, G. L., Hill, T., \& Wyk, J. V. (2003). Distal and proximal factors in domestic violence: A test of an integrated model. Journal of Marriage and Family, 65, 652-667.

Dobash, R. E., \& Dobash, R. (1997). Violence against wives: A case against the patriarchy. New York, NY: The Free Press.

Dobash, R. P., \& Dobash, R. E. (2004). Women's violence to men in intimate relationships. British Journal of Criminology, 44, 324-349.

Dunn, T. J., Baguly, T., \& Brunsden, B. (2013). From alpha to omega: A practical solution to the pervasive problem of internal consistency estimation. The British Journal of Psychology, 105, 399-412. doi:10.1111/bjop.12046

Dutton, D. G. (1994). Patriarchy and wife assault: The ecological fallacy. Violence and Victims, 9, 167-182.

Eastwick, P. W., Eagly, A. H., Glick, P., Johannesen-Schmidt, M. C., Fiske, S. T., Blum, A. M. B., Eckes, T., Freiburger, P., Huang, L., Fernandez, M. L., Manganelli, A. M., Pek, J. C. X., Castro, Y. R., Sakalli-Ugurlu, N., Six-Materna, I., \& Volpato, C. (2006). Is traditional gender ideology associated with sex-typed mate preferences? A test in nine nations. Sex Roles, 54, 603-614. doi:10.1007/s11199-006-9027-x

Eastwick, P. W., \& Finkel, E. J. (2008). Sex differences in mate preferences revisited: Do people know what they initially desire in a romantic partner? Journal of Personality and Social Psychology, 94, 245-264. doi:10.1037/0022-3514.94.2.245

Eastwick, P. W., Luchies, L. B., Finkel, E. J., \& Hunt, L. L. (2013). The predictive validity of ideal partner preferences: A review and meta-analysis. Psychological Bulletin, 140, 623-625. doi:10.1037/a0032432 
Eastwick, P. W., \& Neff, L. A. (2012). Do ideal partner preferences predict divorce? A tale of two metrics. Social Psychological and Personality Science, 3, 667-674. doi:10.1177/1948550611435941

Edwards, J. R. (2001). Ten difference score myths. Organizational Research Methods, 4, 265-287.

Edwards, J. R., \& Lambert, L. S. (2007). Methods for integrating moderation and mediation: A general framework using moderated path analysis. Psychological Methods, 12, 1-22. doi:10.1037/1082-989X.12.1.1

Erdfelder, E., Buchner, A., Faul, F., \& Brandt, M. (2004). GPOWER: Power analysis made easy [Computer software]. Available from http://www.gpower.hhu.de/en.html

Fan, P., \& Marini, M. M. (2000). Influences on gender-role attitudes during the transition to adulthood. Social Science Research, 29, 258-283. doi:10.1006/ssre.1999.0669

Faulkner, R. A., Davey, M., \& Davey, A. (2005). Gender-related predictors of change in marital satisfaction and marital conflict. The American Journal of Family Therapy, 33, 61-83. doi:10.1080/01926180590889211

Feinman, S. (1981). Why is cross-sex-role behavior more approved for girls than for boys? A status characteristic approach. Sex Roles, 7, 289-300.

Feinman, S. (1984). A status theory of the evaluation of sex-role and age-role behavior. Sex Roles, 10, 445-456.

Fischer, A. H., Eagly, A. H., \& Oosterwijk, S. (2013). The meaning of tears: Which sex seems emotional depends on the social context. European Journal of Social Psychology, 43, 505-515. doi10.1002/ejsp.1974

Fischer, A. R., \& Good, G. E. (1997). Men and psychotherapy: An investigation of alexithymia, intimacy, and masculine gender roles. Men and Psychotherapy, 34, 160-170.

Fletcher, G. J. O., \& Kerr, P. S. G. (2010). Through the eyes of love: Reality and illusion in intimate relationships. Psychological Bulletin, 136, 627-658. doi:10.1037/a0019792

Fletcher, G. J. O., \& Simpson, J. A. (2000). Ideal standards in close relationships: Their structure and functions. Current Directions in Psychological Science, 9, 102-105.

Fletcher, G. J. O., Simpson, J. A., \& Thomas, G. (1998). The role of ideals in early relationship development. (Unpublished manuscript). University of Canterbury, New Zealand.

Fletcher, G. J. O., Simpson, J. A., \& Thomas, G. (2000). Ideals, perceptions, and evaluations in early relationship development. Journal of Personality and Social Psychology, 79, 933-940. doi:10.1037//0022-3514.79.6.933

Fletcher, G. J. O., Simpson, J. A., \& Thomas, G. (2000). The measurement of perceived relationship quality components: A confirmatory factor analytic approach. Personality and Social Psychology Bulletin, 26, 340-354.

Fletcher, G. J. O., Simpson, J. A., Thomas, G., \& Giles, L. (1999). Ideals in intimate relationships. Journal of Personality and Social Psychology, 76, 72-89.

Follingstad, D. R., Bradley, R. G., Helff, C. M., \& Laughlin, J. E. (2002). A model for predicting dating violence: Anxious attachment, angry temperament, and need for 
relationship control. Violence and Victims, 17, 35-47.

Follingstad, D. R., Wright, S., Lloyd, S., \& Sebastian, J. A. (1991). Sex differences in motivations and effects in dating violence. Family Relations, 40, 51-57.

Frazier, P. A., \& Esterly, E. (1990). Correlates of relationship beliefs: Gender, relationship experience and relationship satisfaction. Journal of Social and Personal Relationships, 7, 331-352.

George, J., \& Stith, S. M. (2014). An updated view of the feminist view of intimate partner violence. Family Process, 53, 179-193. doi:10.1111/famp.12073

Givertz, M., \& Safford, D. (2001). Longitudinal impact of communication patterns on romantic attachment and symptoms of depression. Current Psychology, 30, 148172. doi:10.1007/s12144-011-9106-1

Good, J. J., \& Sanchez, D. T. (2010). Doing gender for different reasons: Why gender conformity positively and negatively predicts self-esteem. Psychology of Women Quarterly, 34, 203-214. doi:10.1111/j.1471-6402.2010.01562.x

Goode, W. J. (1971). Force and violence in the family. Journal of Marriage and Family, 33, 624-636.

Graham-Kevan, N., \& Archer, J. (2003). Intimate terrorism and common couple violence: A test of Johnson's predictions in four British samples. Journal of Interpersonal Violence, 18, 1247-1270. doi:10.1177/0886260503256656

Greenstein, T. N. (1996). Husbands' participation in domestic labor: Interactive effects of wives' and husbands' gender ideologies. Journal of Marriage and Family, 58, 585-595.

Griffin, D., Murray, S., \& Gonzalez, R. (1999). Different score correlations in relationship research: A conceptual primer. Personal Relationships, 6, 505-518.

Gronemeyer, K. M. (1998). Age-related differences in endorsement in traditional femininity roles using the Femininity Ideology Scale. (Unpublished master's thesis). Florida Institute of Technology, FL.

Hammett, J. F. (2015). A dyadic approach to examine the association between intimate partner violence and romantic relationship satisfaction. (Unpublished master's thesis). San Diego State University, CA.

Hammond, M. D. (2015). Sexism in intimate relationships: The interpersonal sources and consequences of ambivalent sexism. (Unpublished doctoral dissertation). University of Auckland, AUS.

Hammond, M. D., \& Overall, N. C. (2013). Men's hostile sexism and biased perceptions of intimate partners: Fostering dissatisfaction and negative behavior in close relationships. Personality and Social Psychology Bulletin, 39, 1585-1599. doi:10.1177/0146167213499026

Hammond, M. D., \& Overall, N. C. (2013). When relationships do not live up to benevolent ideals: Women's benevolent sexism and sensitivity to relationship problems. European Journal of Social Psychology, 43, 212-223. doi:10.1002/ejsp.1939

Hammond, M. D., \& Overall, N. C. (2014). Endorsing benevolent sexism magnifies willingness to dissolve relationships when facing partner-ideal discrepancies. Personal Relationships, 21, 272-287. doi:10.1111/pere.12031 
Hammond, M. D., Overall, N. C., \& Cross, E. J. (2016). Internalizing sexism in close relationships: The perceived sexism of partner maintains women's endorsement of benevolent sexism. Journal of Personality and Social Psychology, 110, 214238. doi:10.1037/pspi0000043

Hayes, A. F. (2012). PROCESS: A versatile computational tool for observed variable mediation, moderation, and conditional process modeling [White paper]. Retrieved from http://www.afhayes.com/public/process2012.pdf

Hayes, A. F. (2013). Introduction to mediation, moderation, and conditional process analysis: A regression-based approach. New York, NY: The Guilford Press

Hayes, A. F., \& Scharkow, M. (2013). The relative trustworthiness of inferential tests of the indirect effect in statistical mediation analysis: Does method really matter? Psychological Science, 24, 1918-1927. doi:10.1177/0956797613480187

Heilman, M. E. (2001). Description and prescription: How gender stereotypes prevent women's ascent up the organizational ladder. Journal of Social Issues, 57, 657674.

Heilman, M. E., \& Wallen, A. S. (2010). Wimpy and undeserving of respect: Penalties for men's gender-inconsistent success. Journal of Experimental Social Psychology, 46, 664-667.

Hendrick, S. S. (1988). A generic measure of relationship satisfaction. Journal of Marriage and the Family, 50, 93-98.

Herek, G. M. (2000). Sexual prejudice and gender: Do heterosexuals' attitudes toward lesbians and gay men differ? Journal of Social Issues, 56, 251-266.

Higgins, E. T. (1987). Self-discrepancy: A theory relating self and affect. Psychological Bulletin, 94, 319-340.

Holmes, F. (2014). The role of masculine norm conformity in men's and women's relational satisfaction. (Unpublished doctoral dissertation). Deakin University, AUS.

Holtzworth-Munroe, A., Bates, L., Smutzler, N., \& Sandin, E. (1997). A brief review of the research on husband violence: Part I: Maritally violent versus nonviolent men. Aggression and Violent Behavior, 2, 65-99.

Hotaling, G. T., \& Sugarman, D. B. (1986). An analysis of the risk markers in husband to wife violence: The current state of knowledge. Violence and Victims, 1, 101-124.

Hu, L., \& Bentler, P. M. (1999). Cutoff criteria for fit indices in covariance structure analysis: Conventional criteria versus new alternatives. Structural Equation Modeling, 6, 1-55.

Huff, C., \& Tingley, D. (2015). “Who are these people?” Evaluating the demographic characteristics and political preferences of MTurk survey respondents. Research and Politics, 2, 1-12. doi:10.1177/2053168015604648

Hurt, M. M., Nelson, J. A., Turner, D. L., Haines, M. E., Ramsey, L. R., Erchull, M. J., \& Liss, M. (2007). Feminism: What is it good for? Feminine norms and objectification as the link between feminist identity and clinically relevant outcomes. Sex Roles, 57, 355-363. doi:10.1007/s11199-007-9272-7

Impett, E. A., \& Peplau, L. A. (2006). "His" and "her" relationships? A review of the empirical evidence. In A. L. Vangelisti \& D. Perlman (Eds.), The Cambridge 
Handbook of Personal Relationships (273-291). New York, NY: Cambridge University Press.

Janoff-Bulman, R., \& Wade, M. B. (1996). The dilemma of self-advocacy for women: Another case of blaming the victim? Journal of Social and Clinical Psychology, $15,143-152$.

Jaspaert, E. (2015). A dyadic analysis of the role of preference disconfirmation in the explanation of intimate partner violence (Unpublished doctoral dissertation). KU Leuven, Belgium.

Jaspaert, E., \& Vervaeke, G. (2014). Exploring the indirect effect of preference discrepancy on intimate partner violence. Journal of Family Violence, 29, 829837. doi:10.1007/s10896-014-9636-Z

Jenkins, S. S., \& Aube, J. (2002). Gender differences and gender-related constructs in dating aggression. Personality and Social Psychology Bulletin, 28, 1106-1118.

Jewell, J. A., \& Brown, C. S. (2014). Relations among gender typicality, peer relations, and mental health during early adolescence. Social Development, 23, 137-156. doi:10.1111/sode.12042

Johnson, M. P. (1995). Patriarchal terrorism and common couple violence: Two forms of violence against women. Journal of Marriage and the Family, 57, 283-294.

Johnson, M. P. (2006). Conflict and control: Gender symmetry and asymmetry in domestic violence. Violence Against Women, 12, 1003-1018. doi:10.1177/1077801206293328

Johnson, M. P. (2011). Gender and types of intimate partner violence: A response to an anti-feminist literature review. Aggression and Violent Behavior, 16, 289-293. doi:10.1016/j.avb.2011.04.006

Kane, E. W. (2006). "No way my boys are going to be like that!" Parents' responses to children's gender nonconformity. Gender and Society, 20, 149-176. doi: $10.1177 / 0891243205284276$

Kaura, S. A., \& Lohman, B. J. (2007). Dating violence victimization, relationship satisfaction, mental health problems, and acceptability of violence: A comparison of men and women. Journal of Family Violence, 22, 367-381. doi:10.1007/s10896-007-9092-0

Kearney, M. S. (2015). Recognizing warning signs of dating violence: The contributions of gender role orientation and hypergender constructs. (Unpublished Master's thesis). University of Maryland, MD.

Kenny, D. A. (2017). MedPower: An interactive tool for the estimation of power in tests of mediation [Computer software]. Available from https://davidakenny.shinyapps.io/PowerMed/.

Kenny, D. A., \& McCoach, D. B. (2003). Effect of the number of variables on measures of fit in structural equation modeling. Structural Equation Modeling, 10, 333-351.

Khairallah, T. S. Treloar, H. R., \& McCarthy, D. M. (2010, February). The influence of gender role adherence on alcohol use and problems. Poster session presented at the Samuel B. Guze Symposium on Alcoholism, St. Louis, MO.

Kilmartin, C., \& McDermott, R. C. (2016). Violence and masculinities. In Y. J. Wong \& S. R. Wester (Eds.), APA Handbook of Men and Masculinities (615-363). 
Washington, DC: American Psychological Association. doi:10.1037/14594-028

Kimmel, M. S. (2002). "Gender symmetry" in domestic violence: A substantive and methodological research review. Violence Against Women, 8, 1332-1363. doi:10.1177/107780102237407

Kline, R. B. (2011). Principles and practice of structural equation modeling ( $3^{\text {rd }}$ ed.). New York, NY: The Guilford Press.

Kluwer, E., \& Mikula, G. (2003). Gender-related inequalities in the division of family work in close relationships: A social psychological perspective. European Review of Social Psychology, 12, 185-216. doi:10.1080/10463280240000064

Lamke, L. K., Sollie, D. L., Durbin, R. G., \& Fitzpatrick, J. A. (1994). Masculinity, femininity and relationship satisfaction: The mediating role of interpersonal competence. Journal of Social and Personal Relationships, 11, 535-554.

Lavee, Y., \& Katz, R. (2002). Division of labor, perceived fairness, and marital quality: The effect of gender ideology. Journal of Marriage and Family, 64, 27-39.

Lawrence, E., \& Bradbury, T. N. (2007). Trajectories of change in physical aggression and marital satisfaction. Journal of Family Psychology, 21, 236-247. doi:10.1037/0893-3200.21.2.236

Lee, T. L., Fiske, S. T., Glick, P., \& Chen, Z. (2010). Ambivalent sexism in close relationships: (Hostile) power and (benevolent) romance shape relationship ideals. Sex Roles, 62, 583-601. doi:10.1007/s11199-010-9770-x

Lehman, J. P. (2000). A validity study of the Femininity Ideology Scale. (Unpublished Master's thesis). Florida Institute of Technology, FL.

Leonard, K. E., \& Senchak, M. (1996). Prospective prediction of husband marital aggression within newlywed couples. Journal of Abnormal Psychology, 105, 369380.

Levant, R. F., Hall, R. J., \& Rankin, T. J. (2013). Male Role Norms Inventory-Short Form (MRNI-SF): Development, confirmatory factor analytic investigation of structure, and measurement invariance across gender. Journal of Counseling Psychology, 60, 228-238.

Levant, R. F., Hall, R., Weigold, K. I., \& McCurdy, E. R. (2016). Construct validity evidence for the Male Role Norms Inventory-Short Form: A structural equation modeling approach using the bifactor model. Journal of Counseling Psychology, 63, 534-542. doi:10.1037/cou0000171.supp

Levant, R. F., Hirsch, L. S., Celentano, E., Cozza, T. M., Hill, S., MacEachern, M., Marty, N., \& Schnedeker, J. (1992). The male role: An investigation of contemporary norms. Journal of Mental Health \& Counseling, 14, 325-337.

Levant, R. F., \& Richmond, K. (2007). A review of research on masculinity ideologies using the Male Role Norms Inventory. The Journal of Men's Studies, 15, 130-146. doi:10.3149/jms.1502.130

Levant, R., Richmond, K., Cook, S., House, A. T., \& Aupont, M. (2007). The Femininity Ideology Scale: Factor structure, reliability, convergent and discriminant validity, and social contextual variation. Sex Roles, 57, 373-383. doi:10.1007/211199-0079258-5

Levant, R. F., Smalley, K. B., Aupont, M., House, A. T., Richmond, K., \& Noronha, D. 
(2007). Initial validation of the Male Role Norms Inventory-Revised (MRNI-R).

The Journal of Men's Studies, 15, 83-100. doi:10.3149/jms.1501.83

Lye, D. N., \& Biblarz, T. J. (1993). The effects of attitudes toward family life and gender roles on marital satisfaction. Journal of Family Issues, 14, 157-188.

MacCallum, R. C., Browne, M. W., \& Sugawara, H. M. (1996). Power analysis and determination of sample size for covariance structure modeling. Psychological Methods, 1, 130-149.

Mahalik, J. R., Burns, S. M., \& Syzdek, M. (2007). Masculinity and perceived normative health behaviors as predictors of men's health behaviors. Social Science \& Medicine, 64, 2201-2209. doi:10.1016/j.socscimed.2007.02.035

Mahalik, J. R., Locke, B. D., Ludlow, L. H., Diemer, M. A., Scott, R. P. J., Gottfried, M., \& Freitas, G. (2003). Development of the Conformity to Masculine Norms Inventory. Psychology of Men \& Masculinity, 4, 3-25. doi:10.1037/15249220.4.1.3

Mahalik, J. R., Morray, E. B., Coonerty-Femiano, A., Ludlow, L. H., Slattery, S. M., \& Smiler, A. (2005). Development of the Conformity to Feminine Norms Inventory. Sex Roles, 52, 417-435. doi:10.1007/s11199-005-3709-7

Mahalik, J. R., Talmadge, W. T., Locke, B. D., \& Scott, R. P. J. (2005). Using the Conformity to Masculine Norms Inventory to work with men in a clinical setting. Journal of Clinical Psychology, 61, 661-674. doi:10.1002/jclp.20101

Marsh, H. W., Balla, J. R., \& Grayson, D. (2005). Goodness of fit evaluation in structural equation modeling. In A. Maydeu-Olivares \& J. McArdle (Eds.), Psychometrics. A festschrift to Roderick P. McDonald. Mahwah, NJ: Lawrence Erlbaum Associates, Inc.

Marsh, H. W., Hau, K., \& Wen, Z. (2004). In search of Golden Rules: Comment on hypothesis-testing approaches to setting cutoff values for fit indexes and dangers in overgeneralizing Hu and Bentler's (1999) findings. Structural Equation Modeling: A Multidisciplinary Journal, 11, 320-341. doi:10.1207/s15328007sem1103_2

Marshall, A. D., Panuzio, J., Makin-Byrd, K. N., Taft, C. T., \& Holtzworth-Munroe, A. (2011). A multilevel examination of interpartner intimate partner violence and psychological aggression reporting concordance. Behavioral Therapy, 42, 364377. doi:10.1016/j.beth.2010.09.003

Martin, C. (1990). Attitudes and expectations about children with nontraditional and traditional gender roles. Sex Roles, 22, 151-166.

McDonald, R. P. (1999). Test theory: A unified treatment. Mahwah, NJ: Lawrence Erlbaum Associates. doi:10.1111/j.2044-8317.1981.tb00621.x

McGraw, S. L. (2001). Masculinity ideologies, men's relationship behavior, and relationship satisfaction in heterosexual couple relationships. (Unpublished doctoral dissertation). California School of Professional Psychology, CA.

Meade, A. W., \& Craig, S. B. (2012). Identifying careless responses in survey data. Psychological Methods, 17, 437-456. doi:10.1037/a0028085

Moore, T. M., Stuart, G. L., (2005). A review of the literature on masculinity and partner violence. Psychology of Men \& Masculinity, 6, 46-61. doi:10/1037/1524- 
9220.6.1.46

Moss-Racusin, C. A., Phelan, J. E., \& Rudman, L. A. (2010). When men break the gender rules: Status incongruity and backlash against modest men. Psychology of Men \& Masculinity, 11, 140-151. doi:10.1037/a0018093

Murphy, C. M., \& Hoover, S. A. (1999). Measuring emotional abuse in dating relationships as a multifactorial construct. Violence and Victims, 14, 39-53.

Murray, S. L., Holmes, J. G., \& Griffin, D. W. (1996). The benefits of positive illusions: Idealization and the construction of satisfaction in close relationships. Journal of Personality and Social Psychology, 70, 79-98.

Nazroo, J. (1995). Uncovering gender differences in the use of marital violence: The effect of methodology. Sociology, 29, 475-494.

O’Leary, K. D., \& Williams, M. C. (2006). Agreement about acts of aggression in marriage. Journal of Family Psychology, 20, 656-662. doi:10.1037/08933200.20.4.656

O’Neill, O. A., \& O'Reilly III, C. A. (2011). Reducing the backlash effect: Selfmonitoring and women's promotions. Journal of Occupational and Organization Psychology, 84, 1-8. doi:10.1111/j.2044-8325.2010.02008.x

Osborne, J. W. (2010). Improving your data transformations: Applying the Box-Cox transformation. Practical Assessment, Research \& Evaluation, 15(12), 1-9.

Overall, N. C., Fletcher, G. J. O., \& Simpson, J. A. (2006). Regulation processes in intimate relationship: The role of ideal standards. Journal of Personality and Social Psychology, 91, 662-685. doi:10.1037/0022-3514.91.4.662

Overall, N. C., Fletcher, G. J. O., \& Simpson, J. A. (2010). Helping each other grow: Romantic partner support, self-improvement, and relationship quality. Personality and Social Psychology Bulletin, 36, 1496-1513. doi:10.1177/0146167210383045

Overall, N. C., Sibley, C. G., \& Tan, R. (2011). The costs and benefits of sexism: Resistance to influence during relationship conflict. Journal of Personality and Social Psychology, 101, 271-290. doi:10.1037/a0022727

Panuzio, J., \& DiLillo, D. K. (2010). Physical, psychological, and sexual intimate partner aggression among newlywed couples: Longitudinal prediction of marital satisfaction. Journal of Family Violence, 25, 689-699. doi:10.1007/s10896-0109328-2

Parent, M. C. (2012). Handling item-level missing data: Simpler is just as good. The Counseling Psychologist, 41, 568-600. doi:10.1177/0011000012445176

Parent, M. C., \& Moradi, B. (2009). Confirmatory factor analysis of the Conformity to Masculine Norms Inventory and development of the Conformity to Masculine Norms Inventory-46. Psychology of Men \& Masculinity, 10, 175-189. doi:10.1037/a0015481

Parent, M. C., \& Moradi, B. (2010). Confirmatory factor analysis of the Conformity to Feminine Norms Inventory and the development of an abbreviated version: The CFNI-45. Psychology of Women Quarterly, 34, 97-109.

Parent, M. C., \& Moradi, B. (2011). An abbreviated tool for assessing conformity to masculine norms: Psychometric properties of the Conformity to Masculine Norms Inventory-46. Psychology of Men \& Masculinity, 12, 339-353. 
doi:10.1037/a0021904

Paynter, A., \& Leaper, C. (2016). Heterosexual dating double standards in undergraduate women and men. Sex Roles, 75, 393-406. doi:10.1007/s11199-016-0628-8

Peplau, L. A., Hill, C. T., \& Rubin, Z. (1993). Sex role attitudes in dating and marriage: A 15-year follow-up of the Boston Couples Study. Journal of Social Issues, 49, 31-52.

Perrin, P. B., Heesacker, M., Tiegs, T. J., Swan, L. K., Lawrence, A. W., Smith, M. B., Carrillo, R. J., Cawood, R. L., \& Maejia-Millan, C. M. (2011). Aligning Mars and Venus: The social construction and instability of gender differences in romantic relationships. Sex Roles, 64, 613-628. doi:10.1007/s11199-010-9804-4

Perry, A. R., \& Fromuth, M. E. (2005). Courtship violence using couple data. Journal of Interpersonal Violence, 20, 1078-1095. doi:10.1177/0886260505278106

Pleck, J. H., Sonenstein, F. L., \& Ku, L. C. (1998). Masculinity ideology and its correlates. In B. M. Clinchy \& J. K. Norem (Eds.), The gender and psychology reader (308-333). New York, NY: New York University Press.

Powers, T. A., \& Zuroff, D. C. (1988). Interpersonal consequences of overt self-criticism: A comparison with neutral and self-enhancing presentations of self. Journal of Personality and Socia Psychology, 58, 1054-1062.

Preacher, K. J., \& MacCullum, R. C. (2003). Repairing Tom Swift's electric factor analysis machine. Understanding Statistics, 2, 13-43.

Preacher, K. J., Rucker, D. D., \& Hayes, A. F. (2007). Addressing moderated mediation hypotheses: Theory, methods, and prescriptions. Multivariate Behavioral Research, 42, 185-227.

Reidy, D. E., Shirk, S. D., Sloan, C. A., \& Zeichner, A. (2009). Men who aggress against women: Effects of feminine gender role violation on physical aggression in hypermasculine men. Psychology of Men \& Masculinity, 10, 1-12. doi:10.1037/a0014794

Rempala, D. M., Tolman, R. T., Okdie, B. M., \& Ahn, D. (2014). Gender-role egalitarianism predicts desirable traits of potential marriage partners: A crosscultural comparison. Asian Journal of Social Psychology, 17, 325-330. doi:10.1111/ajsp.12073

Renner, L. M., \& Whitney, S. D. (2012). Risk factors for unidirectional and bidirectional intimate partner violence among young adults. Child Abuse \& Neglect, 36, 40-52. doi:10.1016/j.chiabu.2011.07.007

Rice, S., Fallon, B., \& Bambling, M. (2011). Men and depression: The impact of masculine role norms throughout the lifespan. The Australian Educational and Developmental Psychologist, 28, 133-144.

Richards, C., Bouman, W. P., Seal, L., Barker, M. J., Nieder, T. O., \& T'Sjoen, G. (2016). Non-binary or genderqueer genders. International Review of Psychiatry, 28, 95102. doi:10.3109/09540261.2015.1106446

Richardson, D. S., \& Hammock, G. S. (2007). Social context of human aggression: Are we paying too much attention to gender? Aggression and Violent Behavior, 12, 417-426. doi:10.1016/j.a.avb.2006.11.001

Richmond, K., Levant, R., Smalley, B., \& Cook, S. (2015). The Femininity Ideology 
Scale (FIS): Dimensions and its relationship to anxiety and feminine gender role stress. Women \& Health, 55, 263-279. doi:10.1080/03630242.2014.996723

Riggs, D. S., \& O’Leary, K. D. (1996). Aggression between heterosexual dating partners. Journal of Interpersonal Violence, 11, 519-540.

Riggs, D. S., O’Leary, K. D., \& Breslin, F. C. (1990). Multiple correlates of physical aggression in dating couples. Journal of Interpersonal Violence, 5, 61-73.

Ro, E., \& Lawrence, E. (2007). Comparing three measures of psychological aggression: Psychometric properties and differentiation from negative communication. Journal of Family Violence, 22, 575-586. doi:10.1007/s10896-007-9109-8

Rochlen, A. B., \& Mahalik, J. R. (2004). Women's perceptions of male partners' gender role conflict as predictors of psychological well-being and relationship satisfaction. Psychology of Men \& Masculinity, 5, 147-157. doi:10.1037/15249220.5.2.147

Rochlen, A. B., McKelley, R. A., Suizzo, M., \& Scaringi, V. (2008). Predictors of relationship satisfaction, well-being, and life satisfaction among stay-at-home fathers. Psychology of Men \& Masculinity, 9, 17-28. doi:10.1037/15249220.9.1.17

Rudman, L. A. (1998). Self-promotion as a risk factor for women: The costs and benefits of counterstereotypical impression management. Journal of Personality and Social Psychology, 74, 629-645.

Rudman, L. A., \& Fairchild, K. (2004). Reactions to counterstereotypic behavior: The role of backlash in cultural stereotype maintenance. Journal of Personality and Social Psychology, 87, 157-176. doi:10.1037/0022-3514.87.2.157

Rudman, L. A., \& Glick, P. (2001). Prescriptive gender stereotypes and backlash toward agentic women. Journal of Social Issues, 57, 743-762.

Rudman, L. A., \& Mescher, K. (2013). Penalizing men who request a family leave: Is flexibility stigma a femininity stigma? Journal of Social Issues, 69, 322-340.

Rudman, L. A., \& Phelan, J. E. (2007). The interpersonal power of feminism: Is feminism good for romantic relationships? Sex Roles, 57, 787-799. doi:10.1007/s11199-007-9319-9

Sandnabba, N. K., \& Ahlberg, C. (1999). Parents' attitudes and expectations about children's cross-gender behavior. Sex Roles, 40, 249-263.

Sears, D. J. (2011). Men's attitudes and behavioral conformity to traditional norms of masculinity: Associations with entitlement and aggression. (Unpublished doctoral dissertation). Alliant International University, CA.

Sharpe, M. J., \& Heppner, P. P. (1991). Gender role, gender-role conflict, and psychological well-being in men. Journal of Counseling Psychology, 38, 323-330.

Shelton, B. A., \& John, D. (1996). The division of household labor. Annual Review of Sociology, 22, 299-322.

Shortt, J. W., Capaldi, D. M., Kim, H. K., Kerr, C. R., Owen, L. D., \& Feingold, A. (2012). Stability of intimate partner violence by men across 12 years in young adulthood: Effects of relationship transitions. Prevention Science, 13, 360-369. doi:10.1007/s11121-011-0202-0

Siavelis, R. L., \& Lamke, L. K. (1992). Instrumentalness and expressiveness: Predictors 
of heterosexual relationship satisfaction. Sex Roles, 26, 149-159.

Sibley, C. G., \& Overall, N. C. (2011). A dual process motivational model of ambivalent sexism and gender differences in romantic partner preferences. Psychology of Women Quarterly, 35, 303-317. do:10.1177/0361684311401838

Sidelinger, R. J., Frisby, B. N., \& McMullen, A. L. (2009). The decision to forgive: Sex, gender, and the likelihood to forgive partner transgressions. Communication Studies, 60, 164-179. doi:10.1080/10510970902834890

Simpson, J. A., Fletcher, G. J. O., \& Campbell, L. (2001). The structure and function of ideal standards in close relationships. In G. J. O. Fletcher \& M. S. Clark (Eds.), Blackwell Handbook of Social Psychology: Interpersonal Processes (86-126). Malden, MA: Blackwell Publishers Ltd.

Sirin, S. R., McCreary, D. R., \& Mahalik, J. R. (2004). Differential reactions to men and women's gender role transgressions: Perceptions of social status, sexual orientation, and value dissimilarity. The Journal of Men's Studies, 12, 119-132.

Smiler, A. P. (2006). Conforming to masculine norms: Evidence for validity among adult men and women. Sex Roles, 54, 767-775. doi:10.1007/s11199-006-9045-8

Smiler, A. P., \& Gelman, S. A. (2008). Determinants of gender essentialism in college students. Sex Roles, 58, 864-874. doi:10.1007/s11199-008-9402-x

Smiler, A. P., \& Kubotera, N. (2010). Instrumental or expressive? Heterosexual men's expectations of women in two contexts. Men and Masculinities, 12, 565-574. doi:10.1177/1097184X09337112

Smith, M. D. (1990). Patriarchal ideology and wife beating: A test of a feminist hypothesis. Violence and Victims, 5, 257-273.

Smith, T. E., \& Leaper, C. (2005). Self-perceived gender typicality and the peer context during adolescence. Journal of Research on Adolescence, 16, 91-103.

Spence, J. T., \& Helmreich, R. L. (1972). The Attitudes Toward Women Scale: An objective instrument to measure the attitudes toward the rights and roles of women in contemporary society. JSAS Catalog of Selected Documents in Psychology, 2, 667-668.

Stephanou, G. (2012). Romantic relationships in emerging adulthood: Perception-partner ideal discrepancies, attributions, and expectations. Psychology, 3, 150-160. doi:10.4236.psych.2012.32023

Stith, S. M., Green, N. M., Smith, D. B., \& Ward, D. B. (2008). Marital satisfaction and marital discord as risk markers for intimate partner violence: A meta-analytic review. Journal of Family Violence, 23, 149-160. doi:10.1007/s10896-007-9137-4

Stith, S. M., Smith, D. B., Penn, C. E., Ward, D. B., \& Tritt, D. (2004). Intimate partner physical abuse perpetration and victimization risk factors: A meta-analytic review. Aggression and Violent Behavior, 10, 65-98. doi:10.1016/j.avb.2003.09.001

Straus, M. A. (1979). Measuring intrafamily conflict and violence: The Conflict Tactics (CT) Scales. Journal of Marriage and Family, 41, 75-88.

Straus, M. A. (1994). State-to-state differences in social inequality and social bonds in relation to assaults on wives in the United States. Journal of Comparative Family Studies, 25, 7-24.

Straus, M. A., \& Douglas, E. M. (2004). A short form of the Revised Conflict Tactics 
Scales, and typologies for severity and mutuality. Violence and Victims, 19, 507520.

Straus, M. A., Hamby, S. L., Boney-McCoy, S., \& Sugarman, D. B. (1996). The Revised Conflict Tactics Scale (CTS2). Journal of Family Issues, 17, 283-316.

Swan, S. C., \& Snow, D. L. (2003). Behavioral and psychological differences among abused women who use violence in intimate relationships. Violence Against Women, 9, 75-109. doi:10.1177/1077801202238431

Tabachnick, B. G., \& Fidell, L. S. (2007). Using multivariate statistics (5 ${ }^{\text {th }}$ ed.). New Boston, MA: Pearson.

Taft, C. T., Murphy, C. M., King, D. W., Musser, P. H., \& DeDeyn, J. M. (2003). Process and treatment adherence factors in group cognitive-behavioral therapy for partner violence men. Journal of Consulting and Clinical Psychology, 71, 812-820. doi:10.1037/0022-006X.71.4.812

Tager, D., Good, G. E., \& Brammer, S. (2010). "Walking over 'em”: An exploration of relations between emotion dysregulation, masculine norms, and intimate partner abuse in a clinical sample of men. Psychology of Men \& Masculinity, 11, 233-239. doi:10.1037/a0017636

Testa, M., Hoffman, J. H., \& Leonard, K. E. (2011). Female intimate partner violence perpetration: Stability and predictors of mutual and non-mutual aggression across the first year of college. Aggressive Behavior, 37, 362-373. doi:10.1002/ab.20391

Thomae, M., \& Houston, D. M. (2016). The impact of gender ideologies on men's and women's desire for a traditional or non-traditional partner. Personality and Individual Differences, 95, 152-158. doi:10.1016/j.paid.2016.02.026

Thompson, E. H., \& Pleck, J. H. (1986). The structure of male role norms. American Behavioral Scientist, 29, 531-543.

Thornton, B., \& Leo, R. (1992). Gender typing, importance of multiple roles, and mental health consequences for women. Sex Roles, 27, 307-317.

Tokar, D. M., Thompson, M. N., Plaufcan, M. R., \& Williams, C. M. (2007). Precursors of learning experiences in Social Cognitive Career Theory. Journal of Vocational Behavior, 71, 319-339. doi:10.1016/j.jvb.2007.08.002

Tolman, D. L., \& Porche, M. V. (2000). The Adolescent Femininity Ideology Scale: Development and validation of a new measure for girls. Psychology of Women Quarterly, 24, 365-376.

Travaglia, L. K., Overall, N. C., \& Sibley, C. G. (2009). Benevolent and hostile sexism and preferences for romantic partners. Personality and Individual Differences, 47, 599-604. doi:10.1016/j.paid.2009.05.015

Vandello, J. A., \& Cohen, D. (2008). Culture, gender, and men's intimate partner violence. Social and Personality Psychology Compass, 2, 652-667. doi:10.111/j.1751-9004.2008.00080.x

Wade, J. C., \& Donis, E. (2007). Masculinity ideology, male identity, and romantic relationship quality among heterosexual and gay men. Sex Roles, 57, 775-786. doi:10.1007/s11199-007-9303-4 
Weller, C. (2010). The relationship between feminine gender norms conformity and purpose and meaning in life among female college students (Unpublished doctoral capstone). University of Denver, CO.

Windle, C. R., \& Smith, D. A. (2009). Withdrawal moderates the association between husband gender role conflict and wife marital satisfaction. Psychology of Men \& Masculinity, 10, 245-260. doi:10.1037/a0016757

Wong, P. T. P., Kettlewell, G., \& Sproule, C. F. (1985). On the importance of being masculine: Sex role, attribution, and women's career achievement. Sex Roles, 12, 757-769.

Wood, W., Christensen, P. N., Hebl, M. R., \& Rothgerber, H. (1997). Conformity to sextyped norms, affect, and the self-concept. Journal of Personality and Social Psychology, 73, 523-535.

Young, R., \& Sweeting, H. (2004). Adolescent bullying, relationships, psychological well-being, and gender-atypical behavior: A gender diagnosticity approach. Sex Roles, 50, 525-537.

Zinbarg, R. E., Revelle, W., Yovel, I., \& Li, W. (2005). Cronbach's $\alpha$, Revelle's $\beta$, and McDonald's $\omega_{\mathrm{H}}$ : Their relations with each other and two alternative conceptualizations of reliability. Psychometrika, 70, 123-133. doi:10.1007/s11336-003-0974-7 


\section{Appendix A}

Accessing the Partner-Ideals and Partner-Perceptions Measures

The measures created in this manuscript may be used for non-commercial purposes under the condition that results and study manuscripts are shared with the author. For a copy of the partner-ideals and/or partner-perceptions measures, please contact the author or her adviser:

Dr. Sylvia M. F. Kidder

smf.kidder@gmail.com

Dr. Eric Mankowski

Portland State University

mankowskie@pdx.edu 


\section{Appendix B}

Pilot Study Demographic and Survey Quality Questions

\section{$\underline{\text { Relationship characteristics }}$}

1. How long have you been with your current romantic partner?

- Less than 1 month

- 1-3 months

○ 3-6 months

o 6-12 months

○ 1-2 years

- More than 2 years (please specify)

2. Do you live with your romantic partner?

○ Yes

O No

3. Before your current relationship, how many serious romantic relationships had you been in?
○ 0
○ 1
○ 2
- 3 or more

Demographics

4. What program are you enrolled in at PSU? [First pilot study only]

○ Undergraduate

- Post-baccalaureate

- Graduate

- Not applicable [selecting this skips next two questions]

5. What is your major at PSU? [First pilot study only]

6. Are you an international student? [First pilot study only]

○ No

○ Yes

○ Not applicable

7. What is the highest level of education you have already completed?

○ High school diploma

- Associate's or occupational degree

○ Bachelor's degree (BA, BS, etc.)

- Master's degree (MA, MS, etc.)

○ Professional school degree (MD, JD, etc.)

○ Doctorate degree $(\mathrm{PhD}, \mathrm{EdD}$, etc.)

8. What is the highest level of education your partner has completed?

o Some high school or less

- High school diploma

- Associate's or occupational degree

○ Bachelor's degree (BA, BS, etc.) 
- Master's degree (MA, MS, etc.)

- Professional school degree (MD, JD, etc.)

- Doctorate degree (PhD, EdD, etc.)

9. What is your current employment status? Please check all that apply. [Second pilot study only]

○ Employed full-time

- Employed part-time

○ Full-time student

- Part-time student

○ Not employed

10. What is your partner's current employment status? Please check all that apply. [Second pilot study only]

○ Employed full-time

- Employed part-time

o Full-time student

- Part-time student

- Not employed

11. About how much money did you make in the past year? If you're not sure, please make your best guess.

12. About how much money did your partner make in the past year? If you're not sure, please make your best guess.

13. Do you have a problem with drugs or alcohol? [First pilot study only]

○ Yes

○ No

- Not sure

14. Does your partner have a problem with drugs or alcohol? [First pilot study only]

- Yes

○ No

○ Not sure

15. How would you describe your sexual orientation?

- Straight/heterosexual

- Bisexual

- Gay/homosexual

- Other (please describe)

16. What is your race? Please select all that apply.

- White or Caucasian

- Black or African American

- Latino or Hispanic

- Asian

- Pacific Islander

- Native American

- Other (please describe)

17. What is your partner's race? Please select all that apply.

- White or Caucasian 
- Black or African American

- Latino or Hispanic

- Asian

- Pacific Islander

- Native American

O Other (please describe)

Survey quality questions

1. Which of the following statements best describes how you took this survey? [Second pilot study only]

○ I took this survey alone.

- My partner was nearby but couldn't see how I answered.

- My partner was nearby and could see how I answered.

- Other (please describe)

18. Did you encounter any technical issues completing the survey?

- Yes (please describe)

○ No

19. Sometimes people rush through surveys because they're short on time or just not interested in the questions. As researchers, we want to make sure that we are making conclusions based on careful, attentive responding. In your opinion, should we use the data you provided today?

Note: Your answer will NOT affect your [eligibility for extra credit or participation in the drawing for a gift card]/[compensation for this HIT].

○ Yes

- No (comments welcome below)

[First pilot study only]

Thank you for your participation in this study! We are interested in learning more about how attitudes predict certain characteristics of romantic relationships, and we truly appreciate the time and energy you have invested. Please know that you have made a valuable contribution to psychological science.

If anything in the study made you feel uncomfortable or brought up distressing experiences, please don't hesitate to contact Student Health and Counseling at 503.725.2800 or www.pdx.edu/shac/center-for-student-health-and-counseling. You may also wish to contact the National Domestic Violence Hotline at 1-800-799-7233 or www.thehotline.org/.

You are also welcome to contact us with comments, questions, or concerns:

- Professor Eric Mankowski, Principal Researcher: mankowskie@pdx.edu

- Sylvia Kidder, Researcher: sylvia2@pdx.edu

To enter into the drawing for a \$25 giftcard at Amazon.com or to earn extra credit for your participation in this project, please follow the link below. This 
process protects your confidentiality by keeping your survey responses separate from your contact information.

\section{[Survey link]}

[First pilot study only]

Compensation

1. Which option do you prefer?

- I would like to earn extra credit in my class at PSU (and my instructor has offered me this option).

- I would like to enter into a random drawing for a $\$ 25$ gift card to Amazon.com.

2. [If participant selects extra credit] Please answer the following questions so that you can be awarded extra credit. We will provide this information to your instructor before the end of the term.

3. What is your first name?

4. What is your last name?

5. Which class do you want to earn extra credit in? (Example: Research Methods)

6. What is the name of the instructor in whose class you would like to earn extra credit? (Example: Dr. Johnson)

7. Which term are you taking this course?

- Summer 2016

- Fall 2016

8. [If participant selects drawing] Please provide a current email address so that we can contact you if you are randomly selected to win the gift card. We will only use your email address for this purpose.

9. May we contact you regarding possible future studies? If so, please provide your email address below.

[Second pilot study only]

2. To receive compensation for completing this HIT, please type or paste the code below into the MTurk HIT screen and submit: [randomly generated code]

Thank you for participating in this study! You have made a valuable contribution to psychological science.

If anything in the study made you feel uncomfortable or brought up distressing experiences, you can reach the toll-free National Crisis Line at 800-273-8255. You are also welcome to contact the researchers with comments, questions, or concerns:

- Professor Eric Mankowski, Principal Researcher: mankowskie@pdx.edu

- Sylvia Kidder, Co-Principal Researcher: sylvia2@pdx.edu 


\author{
Appendix C \\ First Pilot Study Consent Form
}

PSU Department of Psychology

Consent Information

\title{
Romantic Relationships of PSU Students
}

You are invited to participate in an online survey about your experiences in a current romantic relationship. This research study is being conducted by Professor Eric Mankowski and his graduate student, Sylvia Kidder. In this study, you will be asked questions about yourself, your partner, and your relationship. This survey should take about 15-25 minutes to complete.

Your responses will be kept completely confidential, so please answer each question as honestly as possible. Only the researchers will see your answers. We are interested in discovering patterns across many people rather than focusing on the responses of any particular individual.

You may experience some discomfort answering personal questions about your beliefs or experiences. However, participation is voluntary, and you are free to skip questions or withdraw from the survey at any time. Choosing to forego or stop the study will not adversely affect your relationship with the researchers, the Psychology Department, or Portland State University.

By participating in this study you will be making a valuable contribution to the study of romantic relationships. As a token of our appreciation, you will have an opportunity to enter into a random drawing for one of two $\$ 25$ gift cards to amazon.com or to earn extra credit in certain PSU classes (if your instructor is offering this option) at the end of the study.

If you have any questions or concerns about the study itself, please contact Professor Mankowski (mankowskie@pdx.edu) or Sylvia Kidder (sylvia2@pdx.edu). If you have questions about your rights as a research participant, please contact the PSU Human Subjects Research Review Committee at (503) 725-4288 or visit www.pdx.edu/research.

Your time and participation are greatly appreciated!

By clicking "I agree" below, you are consenting to participate in this study.

- I agree

- I do not agree [Selecting this option will direct participants to the end of the survey.] 


\section{Appendix D}

Pilot Study Eligibility Screening Questions

1. Are you a current student at PSU?

○ Yes

O No

2. What gender do you consider yourself?

○ Male

- Female

- Other [Participant screened out]

3. How old are you? [Participants indicating that they are younger than 18 screened out]

4. What is your relationship status?

- Single (never married) [Participant screened out]

○ In a relationship

- Married or in a domestic partnership

- Divorced or separated [Participant screened out]

- Widowed [Participant screened out]

5. What gender is your romantic partner?

○ Male

- Female

$\circ$ Other

- I have more than one romantic partner.

6. [Displayed if answer to last question is "I have more than one romantic partner"] What gender is the partner in your primary or longest current relationship?

○ Male

- Female

- Other [Participant screened out]

[Participants who were screened out above were shown a message saying "We appreciate your interest in this survey. Unfortunately, you do not meet the preset screening criteria for participants.] 


\section{Appendix E}

\section{Second Pilot Study Eligibility Screening Questions}

Please note: For the sake of simplicity, this survey will refer to your girlfriend/boyfriend, romantic partner, mate, or spouse as your "partner."

1. What gender do you identify as?

- Man

- Woman

- Other [Participant screened out]

2. Do you consider yourself to be transgender?

○ No

- Yes [Participant screened out]

3. How old are you? [Participants younger than 18 screened out]

4. In which country do you currently reside?

- United States of America

- India [Participant screened out]

- Other (please specify) [Participant screened out]

5. What is your relationship status?

- Single (never married) [Participant screened out]

- In a relationship

- Married or in a domestic partnership

- Divorced or separated [Participant screened out]

- Widowed [Participant screened out]

6. What gender is your romantic partner?

- Man [Male participants screened out]

- Woman [Female participants screened out]

- Other [Participant screened out]

- I have more than one romantic partner. [Participant screened out]

7. How old is your partner? [Participants with partners younger than 18 screened out]

8. How long have you been in a romantic relationship with your current partner?

- Less than 6 months [Participants screened out]

○ 6 months -1 year

$\circ$ Longer than 1 year

[Screened-out participants received a message saying "Thank you for your time and interest in this study. Unfortunately, you do not meet the preset screening criteria for participants. Please return the HIT.] 


\author{
Appendix F \\ Second Pilot Study Consent Form \\ Relationship Experiences Study Information
}

\title{
Study Overview
}

You are invited to participate in an online research study about romantic relationships being conducted by Dr. Eric Mankowski and his doctoral student, Sylvia Kidder, at Portland State University. In this study, you will be asked about yourself and your perceptions of your romantic partner and relationship. It should take about 10-20 minutes to complete the survey.

\section{Confidentiality}

Your responses will be kept completely confidential, so please answer each question as honestly as possible. Because your MTurk Worker ID can be linked to your public Amazon.com profile page, you may wish to restrict what information you share publically. We will use your MTurk Worker ID only to award payment and avoid duplicating responses in future research. Your MTurk Worker ID will be stored separately from your survey responses, and we will not include any information in any report that would make it possible to identify you.

\section{Risks and Benefits}

There are no known immediate benefits to you for completing this study, and you may experience some discomfort answering personal questions about your perceptions or experiences. However, participation is voluntary, and you are free to skip questions that make you uncomfortable or to withdraw from the study at any time. Choosing to forego or stop the study will not adversely affect your relationship with the researchers, the Psychology Department, or Portland State University.

By participating in this study you are making a valuable contribution to the study of perceptions in romantic relationships. As a token of our appreciation, you will earn $\$$ \$0.20 through MTurk for successfully completing this study.

\section{Questions}

Please feel free to contact the researchers (mankowskie@pdx.edu or sylvia2@pdx.edu) with questions or concerns about the study. If you have questions about your rights as a research participant, please contact the PSU Human Subjects Research Review Committee at (503) 725-4288 or visit www.pdx.edu/research. You may print or save a copy of this form for your records.

By clicking "I agree" below, you are consenting to participate in this study.

- I agree

- I do not agree

Please enter your MTurk Worker ID: [used to award compensation] 


\section{Appendix G}

Demographic Information

1. What is your sexual orientation?

- Straight/heterosexual

- Bisexual

- Gay/homosexual

- Other (please describe)

2. What is your race? Please check all that apply.

○ Asian

- Black or African American

- Latino or Hispanic

- Native American

- Pacific Islander

- White or Caucasian

- Other (please describe)

3. What is your partner's race? Please check all that apply.

○ Asian

- Black or African American

- Latino or Hispanic

- Native American

- Pacific Islander

- White or Caucasian

- Other (please describe)

4. What is the highest level of education you have completed?

○ High school diploma

- Associate's or occupational degree

- Bachelor's degree (BA, BS, etc.)

- Master's degree (MA, MS, etc.)

- Professional school degree (MD, JD, etc.)

$\circ$ Doctorate degree $(\mathrm{PhD}, \mathrm{EdD}$, etc.)

5. What is the highest level of education your partner has completed?

- Some high school or less

- High school diploma

- Associate's or occupational degree

- Bachelor's degree (BA, BS, etc.)

- Master's degree (MA, MS, etc.)

- Professional school degree (MD, JD, etc.)

- Doctorate degree $(\mathrm{PhD}, \mathrm{EdD}$, etc.)

6. What is your current employment status? Please check all that apply.

○ Employed full-time

- Employed part-time

○ Full-time student

- Part-time student 
- Not employed

7. What is your partner's current employment status? Please check all that apply.

- Employed full-time

- Employed part-time

o Full-time student

- Part-time student

- Not employed

8. About how much money did you make in the past year? If you're not sure, please make your best guess.

9. About how much money did your partner make in the past year? If you're not sure, please make your best guess. 


\section{Appendix H}

Relationship Characteristics

1. Before your current relationship, how many serious romantic relationships had you been in?

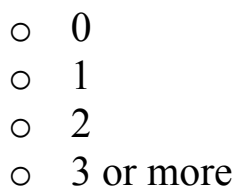

2. Have you ever been (check all that apply)

o Married or in a domestic partnership

○ Divorced or separated

- Widowed

- None of the above

3. How long have you been in a relationship with your current romantic partner? [In years and months]

4. How long have you been formally married or in a domestic partnership? [For married participants]

5. Do you currently live with your romantic partner?

○ Yes

○ No

6. How long have you lived with your partner? [If they live together]

○ Years

○ Months

7. How many children do you have? If you have no children, please enter 0 .

8. How many of your children live with you all or most of the time? [If they have children]

9. How many total children under 18 live in your household (including both your own and others' children)? 


\section{Appendix I \\ Data Quality Questions, Invitation for Partner Survey, and Compensation Directions}

1. Which of the following statements best describes how you took this survey?

○ I took this survey alone.

- My partner was nearby but couldn't see how I answered.

- My partner was nearby and could see how I answered.

- Other (please describe)

2. Did you encounter any technical issues completing this survey?

- Yes (please describe)

○ No

3. Sometimes people rush through surveys because they're busy or just not interested in the questions. As scientists, it is important that we are making conclusions based on careful, attentive responding. In your opinion, should we use the data you provided today?

Your answer will NOT affect your compensation.

- Yes

- No (please explain below)

Inviting Your Partner to Take the Survey

4. Because this study looks at dynamics within couples, we can learn a lot more if your partner also completes the survey. Thus, we would like to ask you to email your partner an invitation to take this survey as well. If your partner successfully completes the survey, they will earn a \$1.50 Amazon.com gift card as a token of our thanks.

Both your responses and your partners' responses would be completely confidential. Your partner would NOT be able to see your answers, and you would NOT be able to see theirs.

Would you be willing to send your partner an invitation to participate in this survey?

$\circ$ Yes (we will give instructions on the next page)

- No (please explain below)

Partner Survey Information

[Only displayed to people who selected "Yes" to the previous question] To participate in the study, your partner will need:

1. The survey link: [survey link]

2. Your Worker ID (this will be used to match your responses to those of your partner while protecting your identities): [MTurk ID]

Below is a template that you can copy and paste into an email:

$* * * * * * * * * * * * * * * * * * * * * * * * * * * * * * * * * * * * * * * * * * * * * * * * * * * * * * * * * * * * * * * * * *$ 
Subject: Invitation to participate in a study on romantic relationships

Hello,

I just completed an online research study on romantic relationships through Amazon's Mechanical Turk. As part of this study, I was asked to invite you to participate so that the researchers could learn more about dynamics within couples.

Here is some more information about the study from the researchers:

This study is being conducted by Professor Eric Mankowski and his graduate student, Sylvia Kidder, from the Psychology Department at Portland State University.

This study asks about yourself, your partner, and experiences in your relationship. It is expected to take about 45-60 minutes. If you participate, your responses will be kept completely confidential. Your partner will NOT be able to see your responses, and you will NOT be able to see theirs. As a token of our appreciation for your time and effort, participants who successfully complete the study will earn a $\$ 1.50$ gift card to Amazon.com.

To begin the survey please click the link below or copy and paste the URL into your browser: [survey link]

To complete the survey, you will need to enter your partner's Amazon Mechanical

Turk Worker ID: [MTurk ID]

It is important for partners to take the survey close together in time, so this survey will close in 2 weeks.

Please click "Next" after you have finished emailing your romantic partner. You will not be able to return to this page.

5. To receive compensation for completing this HIT, please type or paste the code below into the MTurk HIT screen and submit:

[randomly generated code]

Thank you for participating in this study! You have made a valuable contribution to psychological science.

If anything in the study made you feel uncomfortable or brought up distressing experiences, you may wish to call the toll-free National Crisis Line at 800-273-8255 or the National Domestic Violence Hotline at 1-800-799-7233 or www.thehotline.org/. You are also welcome to contact the researchers with comments, questions, or concerns:

- Sylvia Kidder, Co-Principal Researcher: sylvia2@pdx.edu

- Professor Eric Mankowski, Principal Researcher: mankowskie@pdx.edu 


\section{Appendix J \\ Eligibility Screening Pre-Questionnaire}

1. What gender do you identify as?

- Male

- Female

- Other (please describe) [Participant screened out]

2. Do you consider yourself to be transgender?

○ No

- Yes [Participant screened out]

3. How old are you? [Participants younger than 18 screened out]

4. In which country do you currently reside?

- United States of America

- India [Participant screened out]

- Other (please specify) [Participant screened out]

5. What is your relationship status?

- Single (never married) [Participant screened out]

- In a relationship

- Married or in a domestic partnership

- Divorced or separated [Participant screened out]

- Widowed [Participant screened out]

6. What gender is your romantic partner?

- Male [Male participants screened out]

- Female [Female participants screened out]

- Other (please describe) [Participant screened out]

- I have more than one romantic partner. [Participant screened out]

7. Does your romantic partner consider themselves to be transgender?

○ No

- Yes [Participants screened out]

8. How old is your partner? [Participants with partners younger than 18 screened out]

9. How long have you been in a romantic relationship with your current partner?

$\circ \quad$ Less than 6 months [Participants screened out]

○ 6 months -1 year

○ 1 - 5 years

$\circ$ Longer than 5 years

[Screened-out participants received a message saying: "Thank you for your time and interest in this study. Unfortunately, you do not meet the preset screening criteria for participants." MTurk survey participants were then told, "Please return the HIT." Individuals taking the partner survey were told, "Please close this window."] 


\section{Appendix K \\ MTurk Participant Consent Form}

Relationship Experiences Study Information

\section{Study Overview}

You are invited to participate in a research study about romantic relationships being conducted by Professor Eric Mankowski and his doctoral student, Sylvia Kidder, at Portland State University. In this study, you will be asked about yourself, your romantic partner, and experiences in your relationship (including how you and your partner interact and resolve disagreements). The survey is expected to take about 45-60 minutes.

\section{Confidentiality}

We can learn a lot more about dynamics within couples if we hear from both you and your partner. At the end of the survey, you will have the option to email your partner a link to complete the survey as well. If your partner chooses to participate, they will not be able to see your answers, and you will not be able to see theirs. We encourage you to take the survey separately from your partner to maintain privacy. Your responses will be kept completely confidential, so please answer each question as honestly as possible.

We will take measures to protect the confidentiality of our participants. Your MTurk worker ID will be stored separately from your responses (so that we can identify participants who complete similar studies conducted by our lab) and will be permanently deleted from our records after five years. Because your MTurk Worker ID can be linked to your public Amazon.com profile page, you may wish to restrict what information you share publically. We will not be accessing this information, so your responses to all questions will be private and will NOT be shared with external entities. Additionally, we will not include any information in any report we may publish that would make it possible to identify you.

\section{Risks and Benefits}

There are no known immediate benefits to you for completing this study, and you may experience some discomfort answering personal questions about your beliefs or experiences. However, participation is voluntary, and you are free to skip questions that make you uncomfortable or to withdraw from the study at any time. Choosing to forego or stop the study will not adversely affect your relationship with the researchers, the Psychology Department, or Portland State University.

By participating in this study you are making a valuable contribution to the study of romantic relationships. As a token of our appreciation, you will earn $\$ \mathbf{1 . 5 0}$ through MTurk for successfully completing this study. 


\section{Questions}

Feel free to contact us with questions or concerns about the study at sylvia2@pdx.edu or mankowskie@pdx.edu. If you have questions about your rights as a research participant, please contact the PSU Office of Research Integrity, $1600 \mathrm{SW} 4^{\text {th }}$ Avenue, Market Center Building., Ste. 620, Portland, OR 97201; (503) 725-2227 or 1 (877) 4804400; email hsrrc@pdx.edu. Please print or save a copy of this form for your records.

By clicking "I agree" below, you are consenting to participate in this study.

- I agree

- I do not agree [Selecting this option directs participants to the end of the survey.]

Please enter your MTurk Worker ID: 


\author{
Appendix L \\ Partner Survey Consent Form \\ Relationship Experiences Study Information
}

\title{
Study Overview
}

You are invited to participate in a research study about romantic relationships being conducted by Professor Eric Mankowski and his doctoral student, Sylvia Kidder, at Portland State University. In this study, you will be asked about yourself, your romantic partner, and experiences in your relationship (including how you and your partner interact and resolve disagreements). The survey is expected to take about 45-60 minutes. There are 9 short questions to determine eligibility before the study begins.

\section{Confidentiality}

Your romantic partner has already participated in this study. If you participate as well, it will help us learn a lot more about dynamics within couples. Your responses will be kept completely confidential, so please answer each question as honestly as possible. Your partner will NOT be able to see your answers, and you will NOT be able to see theirs. We encourage you to take the survey separately from your partner to maintain privacy.

We will take measures to protect the confidentiality of our participants. All information that you and your partner share will be private, and we NOT share it with external entities. We will not include any information in any report we publish that would make it possible to identify you.

\section{Risks and Benefits}

There are no known immediate benefits to you for completing this study, and you may experience some discomfort answering personal questions about your beliefs or experiences. However, participation is voluntary, and you are free to skip questions that make you uncomfortable or to withdraw from the study at any time. Choosing to forego or stop the study will not adversely affect your relationship with the researchers, the Psychology Department, or Portland State University.

By participating in this study you are making a valuable contribution to the study of romantic relationships. As a token of our appreciation, you will earn a \$1.50 gift card to Amazon.com for successfully completing this study.

\section{Questions}

Feel free to contact us with questions or concerns about the study at sylvia2@pdx.edu or mankowskie@pdx.edu. If you have questions about your rights as a research participant, please contact the PSU Office of Research Integrity, $1600 \mathrm{SW} 4^{\text {th }}$ Avenue., Market Center Building., Ste. 620, Portland, OR 97201; (503) 725-2227 or 1 (877) 4804400; email hsrrc@pdx.edu. Please print or save a copy of this form for your records. 
By clicking "I agree" below, you are consenting to participate in this study.

- I agree

- I do not agree [Selecting this option directs participants to the end of the survey.]

Please enter your romantic partner's Mechanical Turk Worker ID. The Worker ID is a combination of letters and numbers (for example: A2GHP45HVW1) that should be included in the email you received about this study. If you can't find it, please ask your partner to send it to you.

We need this information so that we can match your responses to those of your partner without knowing either of your identities. Your partner will NOT be able to see your responses. 


\section{Appendix M \\ Partner Survey Data Quality Questions and Compensation Directions}

1. Did you encounter any technical issues completing this survey?

- Yes (please explain below)

○ No

2. Sometimes people rush through surveys because they're busy or just not interested in the questions. As scientists, it is important that we are making conclusions based on careful, attentive responding. In your opinion, should we use the data you provided today?

Your answer will NOT affect your compensation.

○ Yes

- No (please explain below)

3. Which of the following statements best describes how you took this survey?

- I took this survey alone.

- My partner was nearby but couldn't see how I answered.

- My partner was nearby and could see how I answered.

- Other (please describe)

4. Please enter the email address where you would like us to send your $\$ 1.50$

Amazon.com gift card. You should receive it within a week. (Your email address will be permanently deleted from our records once data collection has ended).

Thank you for participating in this study! You have made a valuable contribution to psychological science.

If anything in the study made you feel uncomfortable or brought up distressing experiences, you may wish to call the toll-free National Crisis Line at 800-273-8255 or the National Domestic Violence Hotline at 1-800-799-7233 or www.thehotline.org/. You are also welcome to contact the researchers with comments, questions, or concerns:

- Sylvia Kidder, Co-Principal Researcher: sylvia2@pdx.edu

- Professor Eric Mankowski, Principal Researcher: mankowskie@pdx.edu 